\title{
Random-matrix theory of quantum transport
}

\author{
C. W. J. Beenakker
}

Reprint No. 552 from

Reviews

of

Modern

Physics

Volume 69, No. 3, July 1997

Published by The American Physical Society

through the American Institute of Physics 


\section{Revievs Of

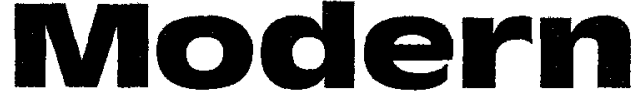 Physics}

\author{
Editorial Office \\ Department of Physics \\ University of Washington \\ Box 351560 \\ Seattle, WA $98195-1560$
}

Fax: (206) 543-6782

E-Mail: rmp@phys.washington.edu

A quarterly journal published by The American Physical Society through the American Institute of Physics.

Reviews of Modern Physics seeks to enhance communication among physicists by publishing comprehensive scholarly reviews of significant topics, as well as colloquia and tutorial articles in rapidly developing fields of physics.

Editor: George F. Bertsch, Department of Physics, University of Washington, Seattle, WA 98195

Colloquium Editor: Anthony F. Starace, Dept. of Physics \& Astronomy, University of Nebraska, Lincoln, NE 68588-0111

Associate Editors:

M. Dine, Department of Physics, University of California, Santa Cruz, CA 95064

S. Haroche, Laboratoire de Physique, École Normale Supérieure, 75231 Paris, France

J. D. Joannopoulos, Department of Physics, Massachusetts Institute of Technology, Cambridge, MA 02139

W. J. Marciano, Physics Department, Brookhaven National Laboratory, Upton, NY 11973

C. F. McKee, Departments of Physics and Astronomy, University of California, Berkeley, CA 94720

K. Nakamura, Institute of Particle and Nuclear Studies, KEK (High Energy Accelerator Research Organization),

Oho, Tsukuba, Japan

H. R. Ott, Laboratorium für Festkörperphysik, ETH-Hönggerberg. $\mathrm{CH}-8093$ Zürich, Switzerland

C. J. Pethick, Nordita, DK-2100 Copenhagen $\emptyset$, Denmark

S. C. Prager, Department of Physics, University of Wisconsin, Madison, WI 53706

A. Richter, Institut für Kernphysik, Technischen Hochschule Darmstadt, D-64289 Darmstadt, Germany

G. Scoles, Department of Chemistry, Princeton University, Princeton, NJ 08544

Assistant Editor: Karie Friedman

American Institute of Physics Editorial Staff: Yin Ping Ho, Thomas Thrash

Subscription Prices (1997)

APS Members

(July 1997-June 1998)

Members of AIP Societies (1997)

$\begin{array}{cc}\text { U.S.A. } & \begin{array}{c}\text { Can., Mex., } \\ \text { Central and }\end{array} \\ \text { and Poss. } & \text { S. Amer., \& Carib. } \\ \$ 50 & \$ 60 \\ \$ 80 & \$ 95\end{array}$

$\begin{array}{cc}\begin{array}{c}\text { Europe, Asia, Africa, and Oceania } \\ \text { Optional } \\ \text { Airfreight }\end{array} \\ \text { Surface } & \$ 80 \\ \$ 60 & \$ 100 \\ \$ 95 & \end{array}$

Additional information regarding subscriptions, reprints, and submission of manuscripts is provided on inside back cover.

Copyright 1997, The American Physical Society. Permission to quote excerpts or to reprint any figures or tables should be obtained directly from an author. The American Physical Society for its part hereby grants permission to quote excerpts and reprint up to 25 figures and/or tables from its journals with acknowledgment of the sources. Requests for permission to reprint in one publication more than 25 figures and/or tables from the totality of APS journals must be made in writing to the Associate Publisher (see address below). A $\$ 10$ fee per figure and/or table in excess of 25 will be charged. Individual teachers, students, researchers, and libraries in nonprofit institutions acting for them are permitted to make hard copies of articles for use in teaching or research, provided such copies are not sold. Copying of articles for sale by document delivery services or suppliers, or beyond the free copying allowed above, is permitted against payment of a copying fee, which is $\$ 10.00$ plus $\$ 0.15$ per page. This fee for document delivery services is shown at the bottom of the first page of each article. Express permission of APS is not required, except for purposes stated below, provided that the fee is paid through the Copyright Clearance Center, Inc. (CCC), 222 Rosewood Drive, Danvers, MA 01923. Contact the CCC for information on how to report copying and remit payments. Reproduction in a reprint collection, or for advertising or promotional purposes, or republication in any form requires permission of one of the authors and a license from APS. Such a license must be sought from Mina Chung, Associate Publisher, The American Physical Society, One Physics Ellipse, College Park, MD 20740-3844. Permission of the author must be obtained from him or her directly. 


\title{
Random-matrix theory of quantum transport
}

\author{
C. W. J. Beenakker \\ Instituut-Lorentz, University of Leiden, 2300 RA Lerden, The Netherlands
}

This is a review of the statistical properties of the scattering matrix of a mesoscopic system Two
geometries are contrasted A quantum dot and a disordered wire The quantum dot is a confined
region with a chaotic classical dynamics, which is coupled to two electron reservoirs via point contacts
The disordered wire also connects two reservoirs, either directly or via a point contact or tunnel
bairier One of the two reservoirs may be in the superconducting state, in which case conduction
involves Andreev reflection at the interface with the superconductor In the case of the quantum dot,
the distribution of the scattering matrix is given by either Dyson's circular ensemble for ballistic point
contacts or the Poisson kernel for point contacts containing a tunnel barrier In the case of the
disordered wire, the distribution of the scattering matrix is obtamed from the
Dorokhov-Mello-Pereyia-Kumar equation, which is a one-dimensional scaling equation The
equivalence is discussed with the nonlinear $\sigma$ model, which is a supersymmetric field theory of
localization The distribution of scattering matrices is applied to a variety of physical phenomena,
1ncluding universal conductance fluctuations, weak localization, Coulomb blockade, sub-Poissonian
shot noise, reflectionless tunneling into a superconductor, and giant conductance oscillations in a
Josephson junction [S0034-6861(97)00203-1]

\section{CONTENTS}

I Introduction

732

A Preface

B Statistical theory of energy levels

1 Wigner Dyson ensemble

2 Geometrical correlations

3 Transition between ensembles

4 Brownian motion

C Statistical theory of transmission eigenvalues

1 Scattering and transfer matrices

2 Linear statistics

3 Geometrical correlations

D Correlation functions

1 Method of functional derivatives

2 Universal conductance fluctuations

E Overview

II Quantum Dots

A Transport theory of a chaotic cavity

1 Crrcular ensemble of scattering matrices

2 Poisson kernel

3 Gaussian ensemble of Hamiltonıans

4 Justification from microscopic theory

B Weak localization

1 Conductance

2 Other transport properties

3 Tunnel barriers

4 Magnetoconductance

C Universal conductance fluctuations

1 Conductance

2 Other transport properties

3 Tunnel barriers

4 Magnetoconductance

D Conductance distribution

E Phase breaking

1 Invasive voltage probe

2 Inelastic scattering

F Coulomb blockade

$\mathrm{G}$ Frequency dependence

III Disordered Wires

A Dorokhov Mello-Pereyra-Kumar equation

1 Scalıng approach to localization

2 Brownian motion of transmission eigenvalues
3 Mapping to a free fermon model

4 Equivalence to a supersymmetric field theory

B Metallic regime

1 Conductance

2 Other transport properties

3 Transmission eigenvalue density

4 Scaling as a hydrodynamic flow

5 Nonlogarithmic eigenvalue repulsion

C Localized regume

1 Log normal distribution of the conductance

2 Crystallization of transmission ergenvalues

D Disordered wire with obstacles

1 Obstacle as initial condition for scaling

2 Point contact

3 Single-channel limit

4 Double-barrier junction

E Shot noise

IV Normal-Metal-Superconductor Junctions

A Scattering theory

1 Andreev reflection

2 Bogoliubov-De Gennes equation

3 Scattering formula for the conductance

B Ideal normal-metal-superconductor interface

1 Average conductance

2 Weak localization

3 Unversal conductance fluctuations

C Normal-metal-superconductor junction contanning a tunnel barner

1 Reflectionless tunneling

2 Scaling theory

3 Double-barrier junction

4 Circuit theory

D Normal-metal-superconductor junction containing a point contact

1 Giant backscattering peak

2 Conductance doubling

E Chaotic Josephson junction

1 Average conductance

2 Conductance fluctuations

F Shot noise

V Conclusion

A Hrgher-dimensional geometries

$B$ Localization of interacting particles

C Localization of light
760 
D. Quantum Hall effect

Acknowledgments

Appendix A: Integral Equation for the Eigcnvalue Density Appendix B: Integration Over The Unitary Group

Appendix C: How to Derive Eq. (194) From Eq. (210)

Appendix D: Calculation of the Weak-Localization Corrections in Table III

1. Broken time-reversal symmetry

2. Broken electron-hole degeneracy

3. Both symmetries broken

4. Effect of spin-orbit scattering

References

\section{INTRODUCTION}

\section{A. Preface}

Random-matrix theory deals with the statistical properties of large matrices with randomly distributed elements. The probability distribution of the matrices is taken as input, from which the correlation functions of eigenvalues and eigenvectors are derived as output. From the correlation functions one then computes the physical properties of the system. Random-matrix theory was developed into a powerful tool of mathematical physics in the 1960's, notably by Wigner, Dyson, Mehta, and Gaudin. (Their work is described in detail in a monograph by Mehta, 1991.) The original motivation for this research was to understand the statistics (in particular the distribution of spacings) of energy levels of heavy nuclei, measured in nuclear reactions (Wigner, 1957). (Many of the early papers have been collected in a book by Porter, 1965.) Later the same techniques were applied to the level statistics of small metal particles, in order to describe the microwave absorption by granular metals (Gor'kov and Eliashberg, 1965). Much of the work on level statistics in nuclear and solid-state physics has been reviewed by Brody et al. (1981).

In recent years there has been a revival of interest in random-matrix theory, mainly because of two developments. The first was the discovery that the WignerDyson ensemble applies generically to chaotic systems (Bohigas, Giannoni, and Schmit, 1984; Berry, 1985). (For reviews of the random-matrix theory of quantum chaos, see Bohigas, 1990; Gutzwiller, 1990; Haake, 1992.) The second was the discovery of a relation between universal properties of large random matrices and universal conductance fluctuations in disordered conductors (Altshuler and Shklovskiǔ, 1986; Imry, 1986a). This led to the development of a random-matrix theory of quantum transport. An influential review of the early work was provided by Stone et al. (1991). The field has matured rapidly since then, and the need was felt for an up-to-date review, in particular for physicists from outside the field. The present article was written with this need in mind.

The random-matrix theory of quantum transport is concerned with mesoscopic systems, at the borderline between the microscopic and the macroscopic world. On the one hand, they are sufficiently small that electrons maintain their quantum-mechanical phase coherence, so that a classical description of the transport properties is inadequate. On the other hand, they are sufficiently large that a statistical description is meaningful. Quantum interference leads to a variety of new phenomena. (For reviews, see Altshuler, Lee, and Webb, 1991; Beenakker and Van Houten, 1991; Datta, 1995; Imry, 1996.) Some of the phenomena are "universal," in the sense that they do not depend on the sample size or the degree of disorder-at least within certain limits. Random-matrix theory relates the universality of transport properties to the universality of correlation functions of transmission eigenvalues. A particularly attractive feature of this approach is its generality. Since it addresses the entire probability distribution of the transmission matrix, it applies to a whole class of transport properties-not just to the conductance. By including Andreev reflection one can treat hybrid structures containing normal metals and superconductors. Furthermore, since the approach is nonperturbative, it provides a unified description of both the metallic and the localized regimes.

There exists at this moment a complete description of the statistics of the transmission matrix for two types of geometries. The first is a confined geometry, the second a wire geometry. The confined geometry consists of a metal grain through which a current is passed via two point contacts. Such a system is sometimes called a "quantum dot," to emphasize the quantum-mechanical phase coherence of the electrons. The wire geometry should have an aspect ratio length/width $\gg 1$. These two geometries are considered separately in Secs. II and III, as far as normal metals are concerned. The new effects which appear due to superconductivity are the subject of Sec. IV. [There is some overlap between Sec. IV and an earlier review by the author (Beenakker, 1995).] In Sec. $\mathrm{V}$ we identify directions for future research and discuss some outstanding problems, in particular the extension of the random-matrix approach to thin-film and bulk geometries (having length $\leqslant$ width). Section $I$ is devoted to an introduction, containing background material and an overview of things to come.

\section{B. Statistical theory of energy levels}

The random-matrix theory of quantum transport is a statistical theory of the transmission eigenvalues of an open system. In contrast, the random-matrix theory established by Wigner and Dyson addresses the statistics of energy levels of a closed system. In this subsection we briefly consider the Wigner-Dyson ensemble of random Hamiltonians and discuss its fundamental ingredientthe hypothesis of geometrical correlations. We also introduce two topics which we will need later on, transitions between ensembles of different symmetry and Brownian motion of energy levels. 
TABLE I. Summary of Dyson's threefold way. The Hermitian matrix $\mathcal{H}$ (and its matrix of eigenvectors $U$ ) are classified by an index $\beta \in\{1,2,4\}$, depending on the presence or absence of time-reversal (TRS) and spin-rotation (SRS) symmetry.

\begin{tabular}{lcccc}
\hline \hline$\beta$ & TRS & SRS & $\mathcal{H}_{n m}$ & $U$ \\
\hline 1 & yes & yes & real & orthogonal \\
2 & no & irrelevant & complex & unitary \\
4 & yes & no & real quaternion & symplectic \\
\hline
\end{tabular}

\section{Wigner-Dyson ensemble}

Wigner and Dyson studied an ensemble of $N \times N$ Hermitian matrices $\mathcal{H}$, with probability distribution of the form

$$
P(\mathcal{H})=c \exp [-\beta \operatorname{Tr} V(\mathcal{H})]
$$

( $c$ is a normalization constant). If the potential $V(\mathcal{H}) \propto \mathcal{H}^{2}$, the ensemble is called Gaussian. Wigner $(1957,1967)$ concentrated on the Gaussian ensemble because it has independently distributed matrix elements (since $\operatorname{Tr} \mathcal{H}^{2}=\operatorname{Tr} \mathcal{H} \mathcal{H}^{\dagger}=\Sigma_{i j}\left|\mathcal{H}_{i j}\right|^{2}$ ), and this simplifies some of the calculations. To make contact with the Hamiltonian of a physical system, the limit $N \rightarrow \infty$ is taken. It turns out that spectral correlations become largely independent of $V$ in this limit, provided one stays away from the edge of the spectrum. This is the celebrated universality of spectral correlations, about which we will say more in Sec. I.D.

The symmetry index $\beta$ counts the number of degrees of freedom in the matrix elements. These can be real, complex, or real quaternion ${ }^{1}$ numbers, corresponding to $\beta=1,2$, or 4 , respectively. Since the transformation $\mathcal{H} \rightarrow U \mathcal{H} U^{-1}$, with $U$ an orthogonal $(\beta=1)$, unitary $(\beta=2)$, or symplectic ${ }^{2}(\beta=4)$ matrix leaves $P(\mathcal{H})$ invariant, the ensemble is called orthogonal, unitary, or symplectic. Physically, $\beta=2$ applies to the case that time-reversal symmetry is broken, by a magnetic field or by magnetic impurities. In the presence of time-reversal symmetry, one has $\beta=1$ if the electron spin is conserved and $\beta=4$ if spin-rotation symmetry is broken (by strong spin-orbit scattering). This classification, due to Dyson (1962d), is summarized in Table I.

We would like to deduce from $P(\mathcal{H})$ what the distribution is of the eigenvalues and eigenvectors of $\mathcal{H}$. Let $\left\{E_{n}\right\}$ denote the set of eigenvalues and $U$ the matrix of eigenvectors, so that ${ }^{3} \mathcal{H}=U \operatorname{diag}\left(E_{1}, E_{2}, \ldots, E_{N}\right) U^{\dagger}$. Since $\operatorname{Tr} V(\mathcal{H})=\Sigma_{n} V\left(E_{n}\right)$ depends only on the eigenvalues, the distribution of Eq. (1) is independent of the

\footnotetext{
${ }^{1}$ A quaternion $q$ is a $2 \times 2$ matrix which is a linear combination of the unit matrix and the three Pauli spin matrices: $q=a \mathbb{1}+i b \sigma_{x}+i c \sigma_{y}+i d \sigma_{z}$. The quaternion is called real if the coefficients $a, b, c$, and $d$ are real numbers.

${ }^{2} \mathrm{~A}$ symplectic matrix is a unitary matrix with real quaternion elements.

${ }^{3}$ If $\beta=4$, the eigenvalue-eigenvector decomposition is $\mathcal{H}=U \operatorname{diag}\left(E_{1} 1, E_{2} \mathrm{I}, \ldots, E_{N} \mathbb{1}\right) U^{\dagger}$, so that each of the $N$ distinct eigenvalues is twofold degenerate (Kramers' degeneracy).
}

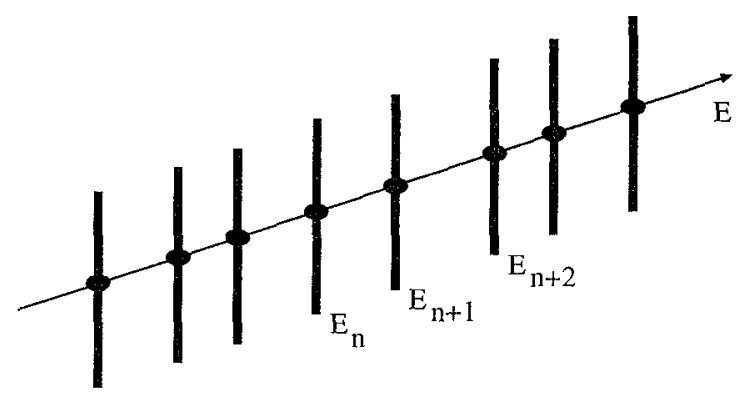

FIG. 1. Schematic illustration of the Coulomb gas. The eigenvalues are represented by classical particles at positions $E_{1}, E_{2}, \ldots, E_{N}$ along a line. The logarithmic eigenvalue repulsion is represented by the Coulomb interaction between identical parallel line charges attached to the particles.

eigenvectors. This means that $U$ is uniformly distributed in the unitary group (for $\beta=2$ ), and in the orthogonal or symplectic subgroups (for $\beta=1$ or 4 ). To find the distribution $P\left(\left\{E_{n}\right\}\right)$ of the eigenvalues we need to multiply $P(\mathcal{H})$ with the Jacobian $J$ which relates an infinitesimal volume element $d \mu(\mathcal{H})$ in the space of Hermitian matrices to the corresponding volume elements $d \mu(U)$, $d E_{n}$ of eigenvectors and eigenvalues,

$$
d \mu(\mathcal{H})=J d \mu(U) \prod_{i} d E_{i}
$$

The Jacobian depends only on the eigenvalues (Porter, 1965),

$$
J\left(\left\{E_{n}\right\}\right)=\prod_{i<j}\left|E_{i}-E_{j}\right|^{\beta} .
$$

The resulting eigenvalue distribution takes the form

$$
P\left(\left\{E_{n}\right\}\right)=c \prod_{i<j}\left|E_{i}-E_{j}\right|^{\beta} \prod_{k} \exp \left[-\beta V\left(E_{k}\right)\right] .
$$

This distribution has the form of a Gibbs distribution in statistical mechanics,

$$
P\left(\left\{E_{n}\right\}\right)=c \exp \left[-\beta\left(\sum_{i<j} u\left(E_{i}, E_{j}\right)+\sum_{i} V\left(E_{i}\right)\right)\right],
$$

where

$$
u\left(E, E^{\prime}\right)=-\ln \left|E-E^{\prime}\right| .
$$

The symmetry index $\beta$ plays the role of inverse temperature. One can imagine that the eigenvalues are classical particles on a line, at the points $E_{1}, E_{2}, \ldots, E_{N}$. They repel each other with a logarithmic pair potential $u$ and are prevented from escaping to infinity by a potential $V$. (For the Gaussian ensemble, $V$ is a parabolic potential well.) This system is called a "Coulomb gas," because the logarithmic repulsion is the Coulomb interaction between two identical parallel line charges (see Fig. 1). The idea of representing the eigenvalue repulsion by a fictitious force is due to Wigner (1957) and Dyson (1962b). It greatly helps our intuition. 


\section{Geometrical correlations}

The fundamental hypothesis ${ }^{4}$ of the Wigner-Dyson ensemble is that spectral correlations are geometrical. Geometrical means that they are due to the Jacobian (3), which relates volume elements in matrix and eigenvalue space. Microscopic details of the system enter only via the potential $V$, which does not by itself create any correlations between the eigenvalues. If there were some other source of correlations, then the interaction $u$ between the eigenvalues would deviate from the logarithmic repulsion given by Eq. (5b). The hypothesis of geometrical correlations is appealing because of its simplicity. Is it correct? In this review we will address that question for the transmission eigenvalues of an open system, where the answer was not known until recently. It is instructive to contrast this with what is known about the energy levels of a closed system.

Gor'kov and Eliashberg (1965) used the WignerDyson ensemble to study the electronic properties of small metal grains. Theoretical justification came with the supersymmetric field theory of Efetov $(1982,1983)$. Assuming diffusive motion of the electrons inside the grain, he obtained the same correlation function of the energy-level density as in the Wigner-Dyson ensemble. Subsequently, Altshuler and Shklovskiu (1986) showed that, for energy separations $\left|E-E^{\prime}\right|$ greater than the Thouless energy $E_{\mathrm{c}}$, the correlation function deviates from random-matrix theory. The characteristic energy scale $E_{\mathrm{c}}=\hbar D / L^{2}$ is inversely proportional to the time it takes for an electron to diffuse, with diffusion coefficient $D$, across a particle of size $L$. It represents the finite width of the energy levels of an open system. The results of the diagrammatic perturbation theory of Altshuler and Shklovskiî were rederived by Argaman, Imry, and Smilansky (1993), using a more intuitive semiclassical method. It follows from these microscopic theories that the repulsion between the energy levels has the logarithmic form of Eq. (5b) of the Wigner-Dyson ensemble for $\left|E-E^{\prime}\right| \ll E_{\mathrm{c}}$. For $\left|E-E^{\prime}\right| \gg E_{\mathrm{c}}$ the interaction potential decays as a power law and actually becomes weakly attractive in three dimensions (Jalabert, Pichard, and Beenakker, 1993).

There is surprisingly little direct experimental evidence for Wigner-Dyson statistics in a metal grain. Sivan et al. (1994) measured the level spacing in a small confined region in a semiconductor (a "quantum dot"). Their results were consistent with Wigner-Dyson statistics for the low-lying excitations. Because of electronelectron interactions, the single-particle excitation spectrum is broadened and merges into a continuum for

\footnotetext{
${ }^{4}$ This viewpoint of what is fundamental in the Wigner-Dyson theory differs from the conventional viewpoint (Porter, 1965) that the two basic assumptions are (1) statistical independence of the matrix elements, and (2) invariance of the ensemble with respect to orthogonal, unitary, or symplectic transformations of $\mathcal{H}$. The assumptions of independence and invariance imply an unnecessary restriction to the Gaussian ensemble.
}

energies further than $E_{\mathrm{c}}$ from the Fermi level (Sivan, Imry, and Aronov, 1994; Altshuler et al. 1996).

The Wigner-Dyson ensemble of random Hamiltonians applies not just to an ensemble of disordered metal grains, but also to any quantum-mechanical system that is sufficiently complex. A necessary requirement is that there are no other constants of the motion than the energy, so no energy level crossings occur. In classical mechanics, such a system is called nonintegrable or chaotic. ${ }^{5}$ Impurity scattering is one way of making the system chaotic, but not the only one. Scattering by the boundaries is often sufficient to destroy all constants of the motion (unless the boundaries have some spatial symmetry). The notion of statistics and averaging is different if the chaos is due to impurity scattering or to boundary scattering. An ensemble of disordered metal grains can be formed by changing the microscopic configuration of the impurities. Alternatively, one could consider a single grain and replace the ensemble average by a spectral average, i.e., by an average over the energy levels. Theory is easier for ensemble averages, whereas experimentally a spectral average is more accessible. The assumption of ergodicity is the assumption that ensemble and spectral averages are equivalent.

Wigner-Dyson statistics of the energy levels has been demonstrated numerically for a variety of nonintegrable systems without disorder, such as a particle moving on a billiard table (Bohigas, Giannoni, and Schmit, 1984), hydrogen in a magnetic field (Freidrich and Wintgen, 1989), and models of strongly interacting electrons (Poilblanc et al., 1993). An early analytical calculation, using periodic-orbit theory, was provided by Berry (1985). A complete theoretical justification, such as Efetov's theory for a disordered grain, was hampered for a long time by the lack of a natural ensemble in the absence of disorder. This obstacle was finally overcome by Andreev et al. (1996). Using a supersymmetric field theory for ballistic motion (Muzykantskiĭ and Khmel'nitskiǐ, 1995), they could show that spectral averages in a chaotic billiard agree with Wigner-Dyson statistics.

\section{Transition between ensembles}

We have talked about time-reversal symmetry as being broken or not. In reality, a weak magnetic field does not break time-reversal symmetry completely. There is a

\footnotetext{
"When speaking of "chaotic" systems, we intend that the classical motion is nonintegrable in the entire phase space (no stable periodic orbits). This is known as "hard" chaos or "global" chaos. Each trajectory then uniformly explores the entire phase space, on a time scale set by the "ergodic" time $\tau_{\text {ergodic }}$. Neighboring trajectories diverge exponentially in time $\propto \exp \left(-t / \tau_{\text {ergodic }}\right)$. It is easy to realize hard chaos in disordered systems, but not in ballistic systems. Generically, the phase space will contain both regions of chaotic and integrable motion ("soft" chaos). Hard chaos has been demonstrated for special geometries in two-dimensional ballistic systems known as "billiards."
} 
smooth transition from the orthogonal or symplectic ensembles to the unitary ensemble. We discuss the transition from the Gaussian orthogonal ensemble (GOE) to the Gaussian unitary ensemble (GUE), following Pandey and Mehta (1983, see also Mehta and Pandey, 1983; Mehta, 1991).

The complex Hermitian $M \times M$ matrix

$$
\mathcal{H}=\mathcal{H}_{0}+i \alpha \mathcal{A}
$$

is decomposed into a real symmetric matrix $\mathcal{H}_{0}$ and a real antisymmetric matrix $\mathcal{A}$ with imaginary weight $i \alpha$. (Here we denote the matrix dimension by $M$ instead of $N$ to avoid a confusion of notation later on in this review.) The two matrices $\mathcal{H}_{0}$ and $\mathcal{A}$ are independently distributed with the same Gaussian distribution, so that the distribution of $\mathcal{H}$ is

$$
P(\mathcal{H}) \propto \exp \left(-\sum_{i, j}\left[\frac{\left(\operatorname{Re} \mathcal{H}_{i j}\right)^{2}}{4 v^{2}}+\frac{\left(\operatorname{Im} \mathcal{H}_{l j}\right)^{2}}{4 v^{2} \alpha^{2}}\right]\right) .
$$

The variance $v^{2}$ determines the mean level spacing $\delta=\pi v / \sqrt{M}$ at the center of the spectrum for $M \gg 1$ and $\alpha \ll 1$. [To have the same mean level spacing for all $\alpha$, one should replace $v^{2}$ by $v^{2}\left(1+\alpha^{2}\right)^{-1}$.] The distribution of $\mathcal{H}$ interpolates between the GOE for $\alpha=0$ and the GUE for $\alpha=1$. The transition is effectively complete for $\alpha \ll 1$. Indeed, the spectral correlations on the energy scale $\delta$ are those of the GUE when the effective strength $v \alpha$ of the term in Eq. (6), which breaks time-reversal symmetry, exceeds $\delta$, hence when $\alpha \geq 1 / \sqrt{M}$.

To relate the parameter $\alpha$ to the magnetic field $B$, we consider the shift $\delta E_{t}$ of the energy levels for $\alpha \ll 1$. On the one hand, from the Hamiltonian (6) one obtains, to leading order in $\alpha$,

$$
\delta E_{l}=\alpha^{2} \sum_{j \neq l} \frac{\mathcal{A}_{t j}^{2}}{E_{t}-E_{j}} .
$$

In order of magnitude, $\left|\delta E_{t}\right| \simeq \alpha^{2} v^{2} / \delta \simeq M \alpha^{2} \delta$. On the other hand, the typical curvature of the energy levels around $B=0$ is given by the Thouless energy: $\left|\delta E_{l}\right|=E_{\mathrm{c}}(e \Phi / h)^{2}$, where $\Phi$ is the magnetic flux through the system. Taken together, these two estimates imply

$$
M \alpha^{2} \simeq\left(\frac{e \Phi}{h}\right)^{2} \frac{E_{\mathrm{c}}}{\delta} \text {. }
$$

The GOE-GUE transition is completed on the energy scale $E$ if $\left|\delta E_{l}\right| \geqslant E$, hence if $\Phi \geqslant(h / e) \sqrt{E / E_{\mathrm{c}}}$. Since $\delta \ll E_{\mathrm{c}}$ in a metal, it requires much less than a flux quantum to break time-reversal symmetry on the scale of the level spacing.

Microscopic justification for the probability distribution of Eq. (7) has been provided by Dupuis and Montambaux (1991) (for a disordered ring) and by Bohigas et al. (1995) (for a chaotic billiard). The precise relation between $\alpha$ and $B$ depends on the geometry of the system and on whether it is disordered or ballistic. For a disordered two-dimensional disk or three-dimensional sphere (radius $R$ much greater than mean free path $l$ ) the relation between $\alpha$ and $\Phi=\pi R^{2} B$ is (Frahm and Pichard, 1995a)

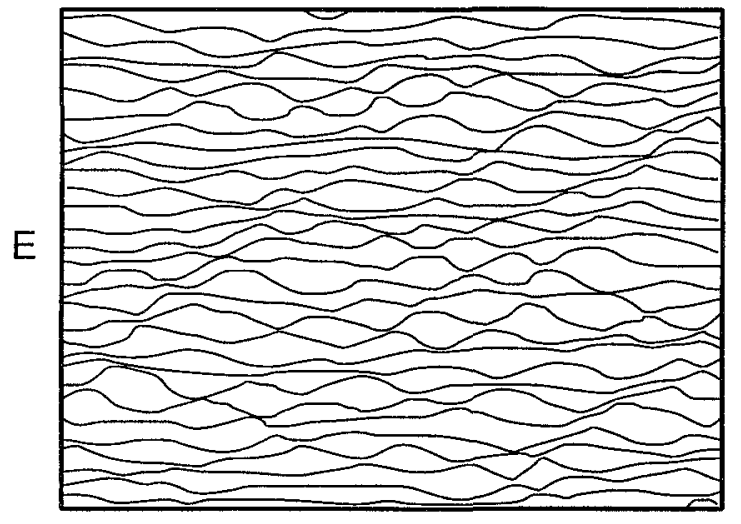

B

FIG. 2. Illustration of the magnetic-field dependence of energy levels in a chaotic system (magnetic field $B$ and energy $E$ in arbitrary units). This plot is based on a calculation of the spectrum of the hydrogen atom in a strong magnetic field by Goldberg et al. (1991).

$$
M \alpha^{2}=\left(\frac{e \Phi}{h}\right)^{2} \frac{\hbar v_{\mathrm{F}} l}{R^{2} \delta} \times \begin{cases}\pi / 4 & \text { disk, } \\ 2 \pi / 15 & \text { sphere. }\end{cases}
$$

Here $v_{\mathrm{F}}$ is the Fermi velocity. For a ballistic disk or sphere $(R \ll l)$, which is chaotic because of diffuse boundary scattering, the relation is instead

$$
M \alpha^{2}=\left(\frac{e \Phi}{h}\right)^{2} \frac{\hbar v_{F}}{R \delta} \times \begin{cases}4 / 3 & \text { disk, } \\ 8 \pi / 45 & \text { sphere }\end{cases}
$$

For a ballistic two-dimensional billiard (area $A$ ) with a chaotic shape, Bohigas et al. (1995) find $M \alpha^{2}$ $=c(e \Phi / h)^{2} \hbar v_{\mathrm{F}} / \delta \sqrt{A}$, with $c$ a numerical coefficient depending only on the shape of the billiard. In each case, $M \alpha^{2} \propto E_{\mathrm{c}}$ in accordance with Eq. (9), the Thouless energy being given by $E_{\mathrm{c}} \simeq \hbar v_{\mathrm{F}} R^{-2} \min (l, R)$.

\section{Brownian motion}

In the previous subsection we considered the magnetic-field dependence of the energy levels around $B=0$, to investigate the transition from the orthogonal to the unitary ensemble. Once the transition is completed, the level distribution becomes $B$ independent. Individual energy levels still fluctuate as a function of $B$ in some random way (see Fig. 2). These spectral fluctuations are a realization of the Brownian-motion process introduced by Dyson (1962c, 1972) as a dynamical model for the Coulomb gas. A review of this topic has been written by Altshuler and Simons (1995). Since it is not directly related to transport, we restrict ourselves here to the basics.

Following Lenz and Haake (1990, see also Haake, 1992), we consider the Hamiltonian

$$
\mathcal{H}=e^{-\tau} \mathcal{H}_{0}+\left(1-e^{-2 \tau}\right)^{1 / 2} \mathcal{H}_{\mathrm{GUE}},
$$

which interpolates between the $M \times M$ complex Hermitian matrices $\mathcal{H}_{0}$ and $\mathcal{H}_{\mathrm{GUE}}$ as the parameter $\tau$ increases 
from 0 to $\infty$. The matrix $\mathcal{H}_{0}$ is a fixed matrix, while $\mathcal{H}_{\mathrm{GUE}}$ varies randomly over the GUE. The resulting distribution of $\mathcal{H}$ is

$$
\begin{aligned}
& P(\mathcal{H}, \tau)=\frac{1}{\left(1-e^{-2 \tau}\right)^{M^{2} / 2}} P_{\mathrm{GUE}}\left(\frac{\mathcal{H}-e^{-\tau} \mathcal{H}_{0}}{\left(1-e^{-2 \tau}\right)^{1 / 2}}\right), \\
& P_{\mathrm{GUE}}(\mathcal{H}) \propto \exp \left(-c \operatorname{Tr} \mathcal{H}^{2}\right) .
\end{aligned}
$$

The coefficients of $\mathcal{H}_{0}$ and $\mathcal{H}_{\text {GUE }}$ in Eq. (12) are chosen such that the mean level spacing $\delta=\pi(2 M c)^{-1 / 2}$ of $\mathcal{H}$ is $\tau$ independent.

The distribution given by Eq. (13) satisfies the Fokker-Planck equation

$$
c \frac{\partial}{\partial \tau} P=\sum_{\mu} \frac{\partial}{\partial \mathcal{H}_{\mu}}\left(c \mathcal{H}_{\mu}+D_{\mu} \frac{\partial}{\partial \mathcal{H}_{\mu}}\right) P
$$

in the $M^{2}$ independent variables $\left\{\mathcal{H}_{\mu}\right\}=\left\{\mathcal{H}_{l l}, \operatorname{Re} \mathcal{H}_{l l}\right.$, $\left.\operatorname{Im} \mathcal{H}_{l j}, \quad 1 \leqslant i<j \leqslant M\right\}$. The diffusion coefficient $D_{\mu}$ equals $1 / 2$ for the diagonal elements $\mathcal{H}_{l l}$ and $1 / 4$ for the off-diagonal elements $\operatorname{Re} \mathcal{H}_{l}, \operatorname{Im} \mathcal{H}_{l]}$. Integrating out the eigenvectors of $\mathcal{H}$, one obtains from Eq. (14) a Fokker-Planck equation for the distribution $P\left(\left\{E_{n}\right\}, \tau\right)$ of the eigenvalues $E_{n}$,

$$
c \frac{\partial}{\partial \tau} P=\sum_{l} \frac{\partial}{\partial E_{l}}\left(c E_{\iota}+\sum_{j \neq l} \frac{1}{E_{J}-E_{l}}+\frac{1}{2} \frac{\partial}{\partial E_{l}}\right) P .
$$

The implication of Eq. (15) is that the energy levels $E_{l}(\tau)$ execute a Brownian motion in fictitious time $\tau$.

To relate $\tau$ to $B$, we first relate $\tau$ to the parameter $\alpha$ of the previous subsection, since we already know how to relate $\alpha$ to $B$. For infinitesimal $\tau$ the Hamiltonian (12) can be written as

$$
\mathcal{H}=\mathcal{H}_{0}+\sqrt{2 \tau}\left(\mathcal{H}_{\mathrm{GOE}}+i \mathcal{A}\right)
$$

Here $\mathcal{H}_{\mathrm{GOE}}$ and $\mathcal{A}$ are, respectively, real symmetric and real antisymmetric matrices having independent Gaussian distributions with the same variance. Equivalently, one can use a purely antisymmetric perturbation of $\mathcal{H}_{0}$ and double its variance:

$$
\mathcal{H}=\mathcal{H}_{0}+2 i \sqrt{\tau} \mathcal{A} \text {. }
$$

Comparison with Eq. (6) leads to the relation (Frahm, 1995b)

$$
\Delta \alpha=2 \sqrt{\tau}
$$

between the fictitious time $\tau$ of the Brownian motion and an increment $\Delta \alpha$ of the Pandey-Mehta Hamiltonian in the absence of time-reversal symmetry (i.e., for $M \alpha^{2} \gg 1$ ). Since $\alpha \propto \Phi$ according to Eq. (9), one finds that $\tau$ is related to the flux increment $\Delta \Phi$ by $M \tau \simeq(e \Delta \Phi / h)^{2} E_{\mathrm{c}} / \delta$.

Microscopic justification for the Brownian-motion model has been provided by Beenakker (1993b), and Beenakker and Rejaei (1994b), through a comparison of the correlation functions obtained from Eq. (15) with those obtained for a disordered metal grain by Szafer and Altshuler (1993) and Simons and Altshuler (1993) and Altshuler and Simons, (1995). The model has one fundamental limitation: Brownian motion correctly de-

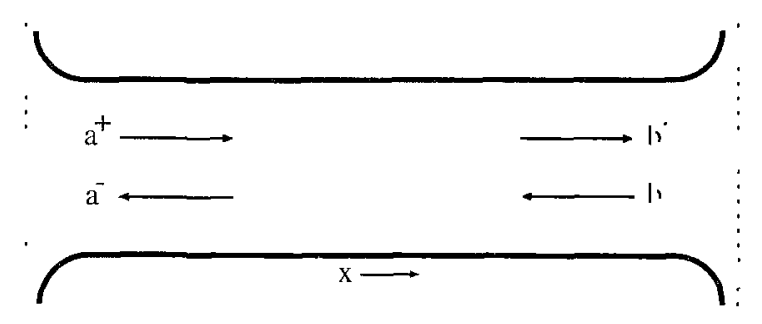

FIG. 3. Disordered region (dotted) connected by ideal leads to two electron reservoirs (to the left and right of the dashed lines). The scattering matrix $S$ relates the amplitudes $a^{+}, b^{-}$of incoming waves to the amplitudes $a^{-}, b^{+}$of outgoing waves, while the transfer matrix $M$ relates the amplitudes $a^{+}, a^{-}$at the left to the amplitudes $b^{+}, b^{-}$at the right.

scribes level correlations between any two values of $B$, but does not describe how levels at three or more values of $B$ are correlated. The reason is that Brownian motion is a Markov process, meaning that it has no memorythe distribution $P$ at time $\tau+\Delta \tau$ is fully determined by the distribution at time $\tau$. Knowledge of $P$ at earlier times is irrelevant for the evolution at later times. The true level dynamics, in contrast, is no Markov process-it does have a memory. To see this, it suffices to take a look at Fig. 2. The energy levels evolve smoothly as a function of magnetic field, hence their location at $B+\Delta B$ is not independent from that at $B-\Delta B$ if $\Delta B$ is small enough. As a consequence, the correlator of two densities $\left\langle n\left(B_{1}\right) n\left(B_{2}\right)\right\rangle$ [with $\left.n(B)=\Sigma_{n} \delta\left(E-E_{n}(B)\right)\right]$ can be obtained from the Fokker-Planck equation (15), but the correlator of three densities $\left\langle n\left(B_{1}\right) n\left(B_{2}\right) n\left(B_{3}\right)\right\rangle$ cannot.

\section{Statistical theory of transmission eigenvalues}

\section{Scattering and transfer matrices}

The scattering theory of electronic conduction is due to Landauer (1957, 1987), Imry (1986b), and Büttiker (1986b, 1988b). It provides a complete description of transport at low frequencies, temperatures, and voltages, under circumstances in which electron-electron interactions can be neglected. (For an overview of the great variety of experiments in which the theory has been tested, see Beenakker and Van Houten, 1991.) A mesoscopic conductor is modeled by a phase-coherent disordered region connected by ideal leads (without disorder) to two electron reservoirs (see Fig. 3). Scattering in the phase-coherent region is elastic. All inelastic scattering is assumed to take place in the reservoirs, which are in equilibrium at zero temperature and electrochemical potential (or Fermi energy) $E_{\mathrm{F}}$. The ideal leads are "electron wave guides," introduced to define a basis for the scattering matrix of the disordered region.

The wave function $\psi$ of an electron in a lead at energy $E_{\mathrm{F}}$ separates into a longitudinal and a transverse part,

$$
\psi_{n}^{ \pm}(\vec{r})=\Phi_{n}(y, z) \exp \left( \pm i k_{n} x\right)
$$


The integer $n=1,2, \ldots, N$ labels the propagating modes, also referred to as scattering channels. Mode $n$ has a real wave number $k_{n}>0$ and transverse wave function $\Phi_{n}$. (We assume, for simplicity of notation, that the two leads are identical.) The normalization of the wave function (19) is chosen such that it carries unit current. A wave incident on the disordered region is described in this basis by a vector of coefficients

$$
c^{\text {in }} \equiv\left(a_{1}^{+}, a_{2}^{+}, \ldots, a_{N}^{+}, b_{1}^{-}, b_{2}^{-}, \ldots, b_{N}^{-}\right) .
$$

The first set of $N$ coefficients refers to the left lead and the second set of $N$ coefficients to the right lead in Fig. 3. Similarly, the reflected and transmitted wave has vector of coefficients

$$
c^{\mathrm{out}} \equiv\left(a_{1}^{-}, a_{2}^{-}, \ldots, a_{N}^{-}, b_{1}^{+}, b_{2}^{+}, \ldots, b_{N}^{+}\right) .
$$

The scattering matrix $S$ is a $2 N \times 2 N$ matrix which relates these two vectors,

$$
c^{\text {out }}=S c^{\text {in }} .
$$

It has the block structure

$$
S=\left(\begin{array}{ll}
r & t^{\prime} \\
t & r^{\prime}
\end{array}\right),
$$

with $N \times N$ reflection matrices $r$ and $r^{\prime}$ (reflection from left to left and from right to right) and transmission matrices $t$ and $t^{\prime}$ (transmission from left to right and from right to left).

Current conservation implies that $S$ is a unitary matrix: $S^{-1}=S^{\dagger}$. It is a consequence of unitarity that the four Hermitian matrices $t t^{\dagger}, t^{\prime} t^{\prime \dagger}, 1-r r^{\dagger}$, and $1-r^{\prime} r^{\prime \dagger}$ have the same set of eigenvalues $T_{1}, T_{2}, \ldots, T_{N}$. Each of these $N$ transmission eigenvalues is a real number between 0 and 1 . The scattering matrix can be written in terms of the $T_{n}$ 's by means of the polar decomposition (Mello, Pereyra, and Kumar, 1988; Martin and Landauer, 1992)

$$
S=\left(\begin{array}{cc}
U & 0 \\
0 & V
\end{array}\right)\left(\begin{array}{cc}
-\sqrt{1-\mathcal{T}} & \sqrt{\mathcal{T}} \\
\sqrt{\mathcal{T}} & \sqrt{1-\mathcal{T}}
\end{array}\right)\left(\begin{array}{cc}
U^{\prime} & 0 \\
0 & V^{\prime}
\end{array}\right) .
$$

Here $U, V, U^{\prime}, V^{\prime}$ are four $N \times N$ unitary matrices and $\mathcal{T}=\operatorname{diag}\left(T_{1}, T_{2}, \ldots, T_{N}\right)$ is a $N \times N$ diagonal matrix with the transmission eigenvalues on the diagonal. ${ }^{6}$

If time-reversal symmetry is broken $(\beta=2)$, unitarity is the only constraint on $S$. The presence of timereversal symmetry imposes additional constraints. If both time-reversal and spin-rotation symmetry are present $(\beta=1)$, then $S$ is unitary and symmetric: $S=S^{\mathrm{T}}$, hence $U^{\prime}=U^{\mathrm{T}}, V^{\prime}=V^{\mathrm{T}}$. (The superscript T indicates the transpose of the matrix.) If time-reversal symmetry is present but spin-rotation symmetry is broken

\footnotetext{
${ }^{6}$ The transmission eigenvalues for $\beta=4$ are twofold degenerate: $\mathcal{T}=\operatorname{diag}\left(T_{1} \rrbracket, T_{2} 1, \ldots, T_{N} \rrbracket\right)$. Compare the footnote on Kramers' degeneracy of the energy levels in Sec. I.B.1.
}

$(\beta=4)$, then $S$ is unitary and self-dual: $S=S^{\mathrm{R}}$, hence $U^{\prime}=U^{\mathrm{R}}, V^{\prime}=V^{\mathrm{R}}$. (The superscript $\mathrm{R}$ indicates the dual ${ }^{7}$ of a quaternion matrix.)

The scattering matrix relates incoming to outgoing states. The transfer matrix relates states in the left lead to states in the right lead. A wave in the left lead is given by the vector of coefficients

$$
c^{\text {left }} \equiv\left(a_{1}^{+}, a_{2}^{+}, \ldots, a_{N}^{+}, a_{1}^{-}, a_{2}^{-}, \ldots, a_{N}^{-}\right) .
$$

The first set of $N$ coefficients refers to incoming waves, the second set of $N$ coefficients to outgoing waves. Similarly, a wave in the right lead has a vector of coefficients

$$
c^{\text {right }} \equiv\left(b_{1}^{+}, b_{2}^{+}, \ldots, b_{N}^{+}, b_{1}^{-}, b_{2}^{-}, \ldots, b_{N}^{-}\right) .
$$

The transfer matrix $M$ is a $2 N \times 2 N$ matrix that relates these two vectors,

$$
c^{\text {right }}=M c^{\text {left }} \text {. }
$$

The scattering and transfer matrices are equivalent descriptions of the disordered region. A convenient property of the transfer matrix is the multiplicative composition rule-the transfer matrix of a number of disordered regions in series (separated by ideal leads) is the product of the individual transfer matrices. The scattering matrix, in contrast, has a more complicated composition rule (containing a matrix inversion). By expressing the elements of $M$ in terms of the elements of $S$ one obtains the polar decomposition of the transfer matrix (Mello, Pereyra, and Kumar, 1988; Mello and Pichard, 1991),

$$
M=\left(\begin{array}{cc}
V & 0 \\
0 & V^{\prime \dagger}
\end{array}\right)\left(\begin{array}{cc}
\sqrt{T^{-1}} & \sqrt{T^{-1}-1} \\
\sqrt{T^{-1}-1} & \sqrt{T^{-1}}
\end{array}\right)\left(\begin{array}{cc}
U^{\prime} & 0 \\
0 & U^{\dagger}
\end{array}\right),
$$

in terms of the same $N \times N$ matrices used in Eq. (24).

Current conservation imposes a "pseudo-unitarity" constraint on the transfer matrix:

$$
S^{-1}=S^{\dagger} \Leftrightarrow \Sigma M^{-1} \Sigma=M^{\dagger}
$$

where $\Sigma$ is a diagonal matrix with $\Sigma_{n n}=1$ for $1 \leqslant n \leqslant N$ and $\Sigma_{n n}=-1$ for $N+1 \leqslant n \leqslant 2 N$. As a consequence, the matrix product $M M^{\dagger}$ and its inverse $\left(M M^{\dagger}\right)^{-1}$ $=\Sigma M M^{\dagger} \Sigma$ have the same set of eigenvalues, or in other words, the eigenvalues of $M M^{\dagger}$ come in inverse pairs. We denote the $2 N$ eigenvalues of $M M^{\dagger}$ by $\exp \left( \pm 2 x_{n}\right)$, with $x_{n} \geqslant 0(n=1,2, \ldots, N)$. By comparing Eqs. (24) and (28) one obtains an algebraic relation between the transfer and transmission matrices (Pichard, 1984),

$$
\left[2+M M^{\dagger}+\left(M M^{\dagger}\right)^{-1}\right]^{-1}=\frac{1}{4}\left(\begin{array}{cc}
t t^{\dagger} & 0 \\
0 & t^{\prime \dagger} t^{\prime}
\end{array}\right),
$$

which implies that the exponent $x_{n}$ is related to the transmission eigenvalue $T_{n}$ by

$$
T_{n}=\frac{1}{\cosh ^{2} x_{n}}
$$

\footnotetext{
${ }^{7}$ The dual $Q^{\mathrm{R}}$ of a matrix $Q$ with quaternion elements $Q_{n m}=a_{n m} \rrbracket+i b_{n m} \sigma_{x}+i c_{n m} \sigma_{y}+i d_{n m} \sigma_{z}$ has elements $Q_{n m}^{\mathrm{R}}$ $=a_{m n} \backslash-i b_{m n} \sigma_{x}-i c_{m n} \sigma_{y}-i d_{m n} \sigma_{z}$.
} 
TABLE II. Symmetry of the scattering matrix $S$ and its matrix of eigenvectors $\Omega$, for the three values of $\beta$.

\begin{tabular}{ccc}
\hline$\beta$ & $S$ & $\Omega$ \\
\hline 1 & unitary symmetric & orthogonal \\
2 & unitary & unitary \\
4 & unitary self-dual & symplectic \\
\hline
\end{tabular}

An altogether different representation of the scattering matrix is the eigenvalue-eigenvector decomposition

$$
S=\Omega \operatorname{diag}\left(e^{i \phi_{1}}, e^{i \phi_{2}}, \ldots, e^{i \phi_{2 N}}\right) \Omega^{\dagger} .
$$

The real numbers $\phi_{n}$ are the scattering phase shifts. (There is a twofold Kramers' degeneracy of the $\phi_{n}$ 's for $\beta=4$.) The $2 N \times 2 N$ unitary matrix $\Omega$ has real elements for $\beta=1$, complex elements for $\beta=2$, and real quaternion elements for $\beta=4$. (Hence $\Omega$ is orthogonal for $\beta=1$ and symplectic for $\beta=4$.) The symmetry properties of the scattering matrix are summarized in Table II. The decomposition (32) mixes states at the left of the disordered region with those at the right and therefore does not distinguish between transmission and reflection. This is why the polar decomposition of Eq. (24) is more suitable for a transport problem. A statistical theory of scattering phase shifts was developed by Dyson (1962a), in the early days of random-matrix theory. Dyson's ensemble of random scattering matrices, known as the circular ensemble, turns out to be the appropriate ensemble for conduction through a quantum dot, as we will discuss in Sec. II.

\section{Linear statistics}

The transmission eigenvalues determine a variety of transport properties. First of all is the conductance $G=\lim _{V \rightarrow 0} \bar{I} / V$, defined as the ratio of the timeaveraged electrical current $\tilde{I}$ through the conductor and the voltage difference $V$ between the two electron reservoirs in the limit of vanishingly small voltage. This is the limit of linear response, to which we restrict ourselves in this review. At zero temperature, the conductance is given by

$$
G=G_{0} \sum_{n=1}^{N} T_{n}, \quad G_{0} \equiv \frac{2 e^{2}}{h} .
$$

Equation (33) is known as the Landauer formula, because of Landauer's pioneering 1957 paper. It was first written down in this form by Fisher and Lee (1981). For an account of the controversy surrounding this formula, which has now been settled, we refer to Stone and Szafer (1988). The factor of two in the definition of the conductance quantum $G_{0}$ is due the twofold spin degeneracy in the absence of spin-orbit scattering. In the presence of spin-orbit scattering, there is a twofold Kramers' degeneracy in zero magnetic field. In the presence of both spin-orbit scattering and a magnetic field, one has a reduced conductance quantum $G_{0}=e^{2} / h$ with twice the number of transmission eigenvalues.
The discreteness of the electron charge causes timedependent fluctuations of the current $I(t)=\bar{I}+\delta I(t)$, which persist down to zero temperature. These fluctuations are known as shot noise. The power spectrum of the noise has the zero-frequency limit

$$
P=4 \int_{0}^{\infty} d t \overline{\delta I\left(t+t_{0}\right) \delta I\left(t_{0}\right)}
$$

where the overline indicates an average over the initial time $t_{0}$ in the correlator. The shot-noise power is related to the transmission eigenvalues by (Büttiker, 1990)

$$
P=P_{0} \sum_{n=1}^{N} T_{n}\left(1-T_{n}\right), \quad P_{0} \equiv 2 e V G_{0} .
$$

Equation (35) is the multichannel generalization of formulas by Khlus (1987) and Lesovik (1989).

More generally, we will study transport properties of the form

$$
A=\sum_{n=1}^{N} a\left(T_{n}\right) .
$$

The quantity $A$ is called a linear statistic on the transmission eigenvalues. The word "linear" indicates that $A$ does not contain products of different eigenvalues, but the function $a(T)$ may well depend nonlinearly on $T$-as it does in the case of the shot-noise power (35), where $a(T)$ depends quadratically on $T$. The conductance (33) is special because it is a linear statistic with a linear dependence on $T$. Other linear statistics [with $a(T)$ a rational or algebraic function] appear if one of the two electron reservoirs is in the superconducting state (see Sec. IV).

\section{Geometrical correlations}

The analogue for random scattering matrices of the Wigner-Dyson ensemble of random Hamiltonians is an ensemble of unitary matrices where all correlations between the transmission eigenvalues are geometrical. Here "geometrical" means due to the Jacobian $J$ which relates the volume elements in the polar decomposition (24),

$$
d \mu(S)=J \prod_{\alpha} d \mu\left(U_{\alpha}\right) \prod_{i} d T_{i} .
$$

The set $\left\{U_{\alpha}\right\}$ is the set of independent unitary matrices in Eq. (24): $\left\{U_{\alpha}\right\}=\{U, V\}$ if $\beta=1$ or $4 ;\left\{U_{\alpha}\right\}$ $=\left\{U, U^{\prime}, V, V^{\prime}\right\}$ if $\beta=2$. The Jacobian depends only on the transmission eigenvalues, ${ }^{8}$

\footnotetext{
${ }^{8}$ For a calculation of the Jacobian (38) from scattering matrix to transmission eigenvalues, see Baranger and Mello (1994), Jalabert, Pichard, and Beenakker (1994), and Jalabert and Pichard (1995). For an earlier, closely related, calculation of the Jacobian from transfer matrix to transmission eigenvalues, see Muttalib, Pichard, and Stone (1987), Mello, Pereyra, and Kumar (1988), and Zanon and Pichard (1988).
} 


$$
J\left(\left\{T_{n}\right\}\right)=\prod_{i<1}\left|T_{t}-T_{\jmath}\right|^{\beta} \prod_{k} T_{k}^{-1+\beta / 2} .
$$

The analogue of the Wigner-Dyson distribution (1),

$$
P(S)=c \exp \left[-\beta \operatorname{Tr} f\left(t t^{\dagger}\right)\right],
$$

yields upon multiplication by $J$ a distribution of the $T_{n}$ 's analogous to Eq. (4),

$$
P\left(\left\{T_{n}\right\}\right)=c \prod_{t<1}\left|T_{t}-T_{j}\right|^{\beta} \prod_{k} T_{k}^{-1+\beta / 2} \exp \left[-\beta f\left(T_{k}\right)\right] .
$$

Muttalib, Pichard, and Stone (1987), and Pichard, Zanon, Imry, and Stone (1990) have based a statistical theory of transmission eigenvalues on this distribution. (Their theory is reviewed by Stone et al., 1991.) To make contact with their work, we perform the change of variables

$$
T_{n}=\frac{1}{1+\lambda_{n}} .
$$

Since $T_{n}$ lies between 0 and 1 , the variable $\lambda_{n}$ ranges from 0 to $\infty$. The distribution (40) transforms to

$$
\begin{aligned}
& P\left(\left\{\lambda_{n}\right\}\right)=c \exp \left[-\beta\left(\sum_{l<1} u\left(\lambda_{l}, \lambda_{\jmath}\right)+\sum_{l} V\left(\lambda_{l}\right)\right)\right] \\
& u\left(\lambda, \lambda^{\prime}\right)=-\ln \left|\lambda-\lambda^{\prime}\right| \\
& V(\lambda)=\left[N-\frac{1}{2}(1-2 / \beta)\right] \ln (1+\lambda)+f\left((1+\lambda)^{-1}\right)
\end{aligned}
$$

Equation (42) has the same form as the Gibbs distribution (5) in the Wigner-Dyson ensemble, with the difference that the $\lambda_{n}$ 's can only take on positive values, while the $E_{n}$ 's are free to range over the whole real axis. All microscopic information about the conductor (its size and degree of disorder) is contained in the confining potential $V(\lambda)$. The hypothesis of geometrical correlations does not specify this function. Muttalib, Pichard, and Stone (1987) have shown that the probability distribution (42) maximizes the entropy of the ensemble, subject to the constraint of a given mean density $\rho(\lambda)$ of the $\lambda_{n}$ 's. The function $V(\lambda)$ is the Lagrange multiplier for this constraint. The Wigner-Dyson ensemble can similarly be interpreted as the ensemble of maximum entropy for a given mean density of states (Balian, 1968).

The correlation functions implied by the probability distribution (42) have been studied for a variety of potentials $V(\lambda)$ by Slevin, Pichard, and Mello (1991), Stone et al. (1991), Chen, Ismail, and Muttalib (1992), Muttalib et al. (1993), and Slevin, Pichard, and Muttalib (1993). It was originally believed that precise agreement with the microscopic theory of a disordered wire could be obtained if only $V(\lambda)$ were properly chosen (Mello and Pichard, 1989). We now know that this is not correct (Beenakker, 1993a). The true eigenvalue repulsion is not logarithmic. In other words, there exist correlations between the transmission eigenvalues over and above those induced by the Jacobian. As we will discuss in Sec.
III.B.5, the hypothesis of geometrical correlations is valid for $T_{n}$ 's close to unity. However, it overestimates the repulsion of smaller $T_{n}$ 's (Beenakker and Rejaei, 1993, 1994a). The appearance of random-matrix ensembles with a nonlogarithmic eigenvalue repulsion is a distinctive feature of the random-matrix theory of quantum transport.

An implication of the nonlogarithmic repulsion is that the true ensemble is not of maximum entropy, at least not in the sense of Muttalib, Pichard, and Stone (1987). We make this qualification because, unlike in statistical mechanics, there is not a single definition of the entropy of a random-matrix ensemble. Slevin and Nagao (1993, 1994) have constructed an alternative maximum-entropy ensemble, in which the repulsion is logarithmic in the variables $x_{n}$ (recall that $T_{n}=1 / \cosh ^{2} x_{n}$ ). The true repulsion, however, is not logarithmic in any variable. It is not known whether there exists some maximum-entropy principle that would produce the correct ensemble for a disordered wire.

\section{Correlation functions}

The established method to compute correlation functions of eigenvalues in the Wigner-Dyson ensemble is the method of orthogonal polynomials (Mehta, 1991). This method works for any dimensionality $N$ of the random matrix but requires a logarithmic repulsion $u\left(\lambda, \lambda^{\prime}\right)=-\ln \left|\lambda-\lambda^{\prime}\right|$ of the eigenvalues. Moreover, although in principle one can assume an arbitrary confining potential, in practice one is restricted in the choice of $V(\lambda)$. (One needs to be able to construct a basis of polynomials which are orthogonal with weight function $e^{-\beta V}$.) For applications to quantum transport one requires a method that is not restricted to a particular $u$ and $V$, but the large- $N$ limit is often sufficient. The method of functional derivatives was developed for such applications (Beenakker, 1993a, 1993c, 1994a). A similar method (for the case of logarithmic repulsion) has been developed in connection with matrix models of quantum gravity (Makeenko, 1991).

\section{Method of functional derivatives}

We consider the two-point correlation function

$$
K\left(\lambda, \lambda^{\prime}\right)=\left\langle\sum_{l, J} \delta\left(\lambda-\lambda_{\imath}\right) \delta\left(\lambda^{\prime}-\lambda_{l}\right)\right\rangle-\rho(\lambda) \rho\left(\lambda^{\prime}\right) .
$$

Here $\rho(\lambda)=\left\langle\Sigma_{l} \delta\left(\lambda-\lambda_{l}\right)\right\rangle$ is the mean eigenvalue density and $\langle\cdots\rangle$ denotes the average with probability distribution (42). Explicitly,

$$
\begin{aligned}
& \rho(\lambda)=\frac{\int d \lambda_{I} \cdots \int d \lambda_{N} e^{-\beta W} \sum_{l} \delta\left(\lambda-\lambda_{l}\right)}{\int d \lambda_{1} \cdots \int d \lambda_{N} e^{-\beta W}}, \\
& W\left(\left\{\lambda_{n}\right\}\right)=\sum_{l<j} u\left(\lambda_{l}, \lambda_{J}\right)+\sum_{l} V\left(\lambda_{l}\right) .
\end{aligned}
$$


The interaction potential $u\left(\lambda, \lambda^{\prime}\right)$ may or may not be logarithmic. By differentiating Eq. (44) we obtain an exact relationship between the two-point correlation function and the functional derivative of the mean density with respect to the confining potential,

$$
K\left(\lambda, \lambda^{\prime}\right)=-\frac{1}{\beta} \frac{\delta \rho(\lambda)}{\delta V\left(\lambda^{\prime}\right)} .
$$

To evaluate this functional derivative we must know how the density depends on the potential. This is a classic problem in random-matrix theory. In the large- $N$ limit the solution is given by the integral equation (Wigner, 1957)

$$
V(\lambda)+\int_{\lambda_{-}}^{\lambda_{+}} d \lambda^{\prime} u\left(\lambda, \lambda^{\prime}\right) \rho\left(\lambda^{\prime}\right)=\text { constant }
$$

where "constant" means independent of $\lambda$ inside the interval $\left(\lambda_{-}, \lambda_{+}\right)$, where $\rho>0$. The boundaries $\lambda_{ \pm}$of the spectrum can be either fixed or free. A fixed boundary is independent of $V$. (An example is the constraint $\lambda>0$.) A free boundary is to be determined selfconsistently from Eq. (47), by requiring that $\rho$ vanishes at the boundary. A free boundary thus depends on $V$. Equation (47) has the "mechanical equilibrium" interpretation that the density $\rho$ adjusts itself to the potential $V$ in such a way that the total force at any point vanishes. The support of $\rho$ is therefore an equipotential. Finite- $N$ corrections to Eq. (47) are smaller by an order $N^{-1}$ for $\beta=1$ or 4 , and by an order $N^{-2}$ for $\beta=2$ (Dyson, 1972; see Appendix A). A rigorous proof, containing precise conditions on $u$ and $V$, has been given by Boutet de Monvel, Pastur, and Shcherbina (1995).

Variation of Eq. (47) gives $^{9}$

$$
\delta V(\lambda)+\int_{\lambda_{-}}^{\lambda_{+}} d \lambda^{\prime} u\left(\lambda, \lambda^{\prime}\right) \delta \rho\left(\lambda^{\prime}\right)=\text { constant }
$$

with the constraint

$$
\int_{\lambda_{-}}^{\lambda_{+}} d \lambda \delta \rho(\lambda)=0
$$

(since the variation of $\rho$ is to be carried out at constant $N)$. The inverse of Eq. (48) is

$$
\begin{aligned}
& \delta \rho(\lambda)=-\int_{\lambda_{-}}^{\lambda_{+}} d \lambda^{\prime} u^{\operatorname{lnv}}\left(\lambda, \lambda^{\prime}\right) \delta V\left(\lambda^{\prime}\right) \\
& \int_{\lambda_{-}}^{\lambda_{+}} d \lambda^{\prime \prime} u\left(\lambda, \lambda^{\prime \prime}\right) u^{\mathrm{inv}}\left(\lambda^{\prime \prime}, \lambda^{\prime}\right)=\delta\left(\lambda-\lambda^{\prime}\right)-\frac{1}{\lambda_{+}-\lambda_{-}} .
\end{aligned}
$$

Equation (49b) means that the integral kernel $u^{\mathrm{inv}}$ is the inverse of $u$ for functions $f(\lambda)$ restricted by $\int d \lambda f=0$.

\footnotetext{
${ }^{9}$ Variation of the boundary $\lambda_{ \pm}$of the spectrum gives an additional contribution $\pm \delta \lambda_{ \pm} \rho\left(\lambda_{ \pm}\right) u\left(\lambda, \lambda_{ \pm}\right)$. This contribution vanishes, either because $\delta \lambda_{ \pm}=0$ (fixed boundary) or because $\rho\left(\lambda_{ \pm}\right)=0$ (free boundary). Variation of the $\lambda$-independent right-hand side of Eq. (47) gives some other $\lambda$-independent constant, not necessarily equal to zero.
}

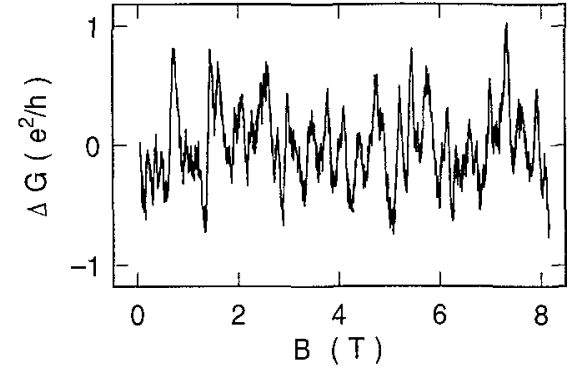

FIG. 4. Fluctuations as a function of perpendicular magnetic field of the conductance of a $310 \mathrm{~nm}$ long and $25 \mathrm{~nm}$ wide $\mathrm{Au}$ wire at $10 \mathrm{mK}$. The trace appears random but is completely reproducible from one measurement to the next. The root mean square of the fluctuations is $0.3 e^{2} / h$, which is not far from the theoretical result $\sqrt{1 / 15} e^{2} / h$ [Eq. (51) with $\beta=2$ due to the magnetic field and a reduced conductance quantum of $e^{2} / h$ due to the strong spin-orbit scattering in Au]. After Washburn and Webb (1986).

Combination of Eqs. (46) and (49) yields a relation between the two-point correlation function and the inverse of the interaction potential (Beenakker, 1993a),

$$
K\left(\lambda, \lambda^{\prime}\right)=\frac{1}{\beta} u^{\mathrm{inv}}\left(\lambda, \lambda^{\prime}\right) .
$$

This relation is universal in that it does not contain the confining potential explicitly. There is an implicit dependence on $V$ through $\lambda_{ \pm}$in Eq. (49), but this can be neglected far from a free boundary. There exists a variety of other demonstrations of such insensitivity of correlation functions to the choice of confining potential (Kamien, Politzer, and Wise, 1988; Ambjørn, Jurkiewicz, and Makeenko, 1990; Ambjørn and Makeenko, 1990; Pastur, 1992; Brézin and Zee, 1993, 1994; Eynard, 1994; Forrester, 1995; Hackenbroich and Weidenmüller, 1995; Kobayakawa, Hatsugai, Kohmoto, and Zee, 1995; Morita, Hatsugai, and Kohmoto, 1995; Akamann and Ambjorn, 1996; Freilikher, Kanzieper, and Yurkevich, 1996).

A universal two-point correlation function implies universal fluctuations of linear statistics, as we discuss in the next subsection.

\section{Universal conductance fluctuations}

Quantum interference leads to significant sample-tosample fluctuations in the conductance at low temperatures. These fluctuations can also be observed in a single sample as a function of magnetic field, since a small change in field has a similar effect on the interference pattern as a change in impurity configuration. Experimental data by Washburn and Webb (1986) for an Au wire at $10 \mathrm{mK}$ is shown in Fig. 4. The fluctuations are not time-dependent noise, but completely reproducible. Such a magnetoconductance trace is called a "magnetofingerprint," because the pattern is specific for the particular sample being studied. Notice that the magnitude of the fluctuations is of order $e^{2} / h$. This is not accidental. 
The universality of the conductance fluctuations was discovered theoretically by Altshuler (1985) and Lee and Stone (1985). There are two aspects to the universality: (1) the variance Var $G$ of the conductance is of order $\left(e^{2} / h\right)^{2}$, independent of sample size or disorder strength; (2) Var $G$ decreases by precisely a factor of two if time-reversal symmetry is broken by a magnetic field. The Altshuler-Lee-Stone theory is a diagrammatic perturbation theory for a disordered metal. Two classes of diagrams, cooperons and diffusons, contribute equally to the variance in the presence of time-reversal symmetry. A magnetic field suppresses the cooperons but leaves the diffusons unaffected, hence the factor-of-two reduction. (We are assuming here, for simplicity, that there is no spin-orbit scattering.) The variance $\operatorname{Var} G / G_{0}$ of the conductance (in units of the conductance quantum $\left.G_{0}=2 e^{2} / h\right)$ is a number of order unity which is weakly dependent on the shape of the conductor. For a wire geometry (length much greater than width) at zero temperature, the variance is

$$
\operatorname{Var} G / G_{0}=\frac{2}{15} \beta^{-1} \text {. }
$$

There is no dependence on the mean free path $l$, the wire length $L$, or the number of transverse modes $N$, provided $l \ll L \ll N l$. That is to say, the wire should be much longer than the mean free path but much shorter than the localization length. The Altshuler-Lee-Stone theory has been tested in many experiments (for reviews, see Altshuler, Lee, and Webb, 1991; Beenakker and Van Houten, 1991).

Shortly after the discovery of the universality of conductance fluctuations, an explanation was given in terms of the repulsion of energy levels (Altshuler and Shklovskiǔ, 1986) or of transmission eigenvalues (Imry, 1986a). Imry's argument contrasts "closed" and "open" scattering channels. Most transmission eigenvalues in a disordered conductor are exponentially small. These are the closed channels. A fraction $l / L$ of the total number $N$ of transmission eigenvalues is of order unity. These are the open channels. Only the open channels contribute effectively to the conductance: $G / G_{0} \equiv N$ eff $\approx N l / L$. Fluctuations in the conductance can be interpreted as fluctuations in the number $N_{\text {eff }}$ of open channels. The alternative argument of Altshuler and Shklovskiı is based on Thouless' (1977) relationship $N_{\text {eff }} \approx E_{\mathrm{c}} / \delta$. (The Thouless energy $E_{\mathrm{c}}$ was defined in Sec. I.B.2; $\delta$ is the mean level spacing.) Conductance fluctuations can be interpreted as fluctuations in the number of energy levels in an energy range $E_{\mathrm{c}}$. If the transmission eigenvalues or energy levels were uncorrelated, one would estimate that fluctuations in $N_{\text {eff }}$ would be of order $\sqrt{N_{\text {eff }}}$. This would imply that $\operatorname{Var} G / G_{0}$ would be of order $N_{\text {eff }}$, which is $\gg 1$. The fact that the variance is of order unity is a consequence of the strong suppression of the fluctuations in $N_{\text {eff }}$ by eigenvalue repulsion.

This argument can be made quantitative. Take a linear statistic

$$
A=\sum_{n=1}^{N} a\left(\lambda_{n}\right) .
$$

For the conductance, we would have $a(\lambda)=(1+\lambda)^{-1}$ [see Eqs. (33) and (41)]. The average of $A$,

$$
\langle A\rangle=\int_{\lambda_{-}}^{\lambda_{+}} d \lambda a(\lambda) \rho(\lambda)
$$

diverges for $N \rightarrow \infty$. We can identify $\langle A\rangle \equiv N_{\text {eff }}$. The variance $\operatorname{Var} A=\left\langle A^{2}\right\rangle-\langle A\rangle^{2}$ is obtained from a double integration of the two-point correlation function (43),

$$
\operatorname{Var} A=\int_{\lambda_{-}}^{\lambda_{+}} d \lambda \int_{\lambda_{-}}^{\lambda_{+}} d \lambda^{\prime} a(\lambda) a\left(\lambda^{\prime}\right) K\left(\lambda, \lambda^{\prime}\right)
$$

For independent $\lambda_{n}$ 's, we would expect $\operatorname{Var} A$ to be of order $N_{\text {eff }}$, so that it too would diverge with $N$. Instead, Eq. (50) implies that

$$
\operatorname{Var} A=\frac{1}{\beta} \int_{\lambda_{-}}^{\lambda_{+}} d \lambda \int_{\lambda_{-}}^{\lambda_{+}} d \lambda^{\prime} a(\lambda) a\left(\lambda^{\prime}\right) u^{\mathrm{inv}}\left(\lambda, \lambda^{\prime}\right),
$$

with corrections of order $1 / N_{\text {eff }}$. This tells us that Var $A$ for large $N$ is independent of $N$, provided the interaction potential $u\left(\lambda, \lambda^{\prime}\right)$ is $N$ independent. Moreover, $\operatorname{Var} A \propto 1 / \beta$ if $u$ is $\beta$ independent. These are the two aspects of universality mentioned above. Let us illustrate this general result by two examples (Beenakker, 1993a, 1993c).

The first example is the Wigner-Dyson ensemble (5), with a logarithmic repulsion. The eigenvalues are free to vary over the whole real axis, hence the end points $\lambda_{ \pm}$of the spectrum are free boundaries. Let us assume that the function $a(\lambda)$ is nonzero only for $\lambda$ in the bulk of the spectrum, so that the integrals from $\lambda_{-}$to $\lambda_{+}$may be replaced by integrals from $-\infty$ to $+\infty$. To determine the functional inverse of $u\left(\lambda, \lambda^{\prime}\right)=-\ln \left|\lambda-\lambda^{\prime}\right|$ in the bulk of the spectrum, we need to solve the integral equation

$$
-\int_{-\infty}^{\infty} d \lambda^{\prime \prime} \ln \left|\lambda-\lambda^{\prime \prime}\right| u^{\mathrm{inv}}\left(\lambda^{\prime \prime}, \lambda^{\prime}\right)=\delta\left(\lambda-\lambda^{\prime}\right) .
$$

This is readily solved by Fourier transformation, with the result

$$
u^{\text {inv }}\left(\lambda, \lambda^{\prime}\right)=-\frac{1}{\pi^{2}} \frac{\partial}{\partial \lambda} \frac{\partial}{\partial \lambda^{\prime}} \ln \left|\lambda-\lambda^{\prime}\right| .
$$

Substitution into Eq. (55) yields a formula for the variance of a linear statistic,

$$
\begin{aligned}
\operatorname{Var} A= & -\frac{1}{\beta \pi^{2}} \int_{-\infty}^{\infty} d \lambda \int_{-\infty}^{\infty} d \lambda^{\prime}\left(\frac{d a(\lambda)}{d \lambda}\right)\left(\frac{d a\left(\lambda^{\prime}\right)}{d \lambda^{\prime}}\right) \\
& \times \ln \left|\lambda-\lambda^{\prime}\right|,
\end{aligned}
$$

or in an equivalent Fourier representation,

$$
\begin{aligned}
& \operatorname{Var} A=\frac{1}{\beta \pi^{2}} \int_{0}^{\infty} d k|a(k)|^{2} k, \\
& a(k)=\int_{-\infty}^{\infty} d \lambda e^{i k \lambda} a(\lambda) .
\end{aligned}
$$


Equation (58) was first derived for the Gaussian ensemble, $V(\lambda) \propto \lambda^{2}$, by Dyson and Mehta (1963; Mehta, 1991). Note that $\operatorname{Var} A$ diverges logarithmically for a step function $a(\lambda)=\theta\left(\lambda-\lambda_{\mathrm{c}}\right)$. More generally, if $a(\lambda)$ changes abruptly on the scale of the eigenvalue spacing, its variance does not have a universal $N \rightarrow \infty$ limit. All physical quantities which we will consider, however, are smooth functions of $\lambda$.

The second example is the ensemble (42) of Muttalib, Pichard, and Stone (1987), relevant for transport properties. The repulsion is still logarithmic, but the eigenvalues are constrained by $\lambda_{n}>0$. Thus $\lambda_{-}=0$ is a fixed lower bound of the spectrum. There is also a free upper bound at some $\lambda_{+} \gg 1$, which does not affect transport properties and can be ignored. (Recall that large $\lambda$ corresponds to small $T$.) Instead of Eq. (56) we now have the integral equation

$$
-\int_{0}^{\infty} d \lambda^{\prime \prime} \ln \left|\lambda-\lambda^{\prime \prime}\right| u^{\mathrm{inv}}\left(\lambda^{\prime \prime}, \lambda^{\prime}\right)=\delta\left(\lambda-\lambda^{\prime}\right)
$$

which can be solved by Mellin transformation. (The Mellin transform is a Fourier transform with respect to the variable $\ln \lambda$.) The result is

$$
u^{\text {inv }}\left(\lambda, \lambda^{\prime}\right)=-\frac{1}{\pi^{2}} \frac{\partial}{\partial \lambda} \frac{\partial}{\partial \lambda^{\prime}} \ln \left|\frac{\sqrt{\lambda}-\sqrt{\lambda^{\prime}}}{\sqrt{\lambda}+\sqrt{\lambda^{\prime}}}\right| .
$$

Instead of Eq. (58) we obtain the formula (Beenakker, 1993a, 1993c; see also Basor and Tracy, 1993; Jancovici and Forrester, 1994)

$$
\begin{aligned}
\operatorname{Var} A= & -\frac{1}{\beta \pi^{2}} \int_{0}^{\infty} d \lambda \int_{0}^{\infty} d \lambda^{\prime}\left(\frac{d a(\lambda)}{d \lambda}\right) \\
& \times\left(\frac{d a\left(\lambda^{\prime}\right)}{d \lambda^{\prime}}\right) \ln \left|\frac{\sqrt{\lambda}-\sqrt{\lambda^{\prime}}}{\sqrt{\lambda}+\sqrt{\lambda^{\prime}}}\right|,
\end{aligned}
$$

or equivalently,

$$
\begin{aligned}
& \operatorname{Var} A=\frac{1}{\beta \pi^{2}} \int_{0}^{\infty} d k|\tilde{a}(k)|^{2} k \tanh (\pi k), \\
& \tilde{a}(k)=\int_{0}^{\infty} d \lambda \lambda^{i k-1} a(\lambda) .
\end{aligned}
$$

The difference between Eqs. (58) and (61) originates entirely from the positivity constraint on $\lambda$ in the transport problem.

Substitution of $a(\lambda)=(1+\lambda)^{-1}$ into Eq. (61) yields the variance of the conductance

$$
\operatorname{Var} G / G_{0}=\frac{1}{8} \beta^{-1}
$$

which differs slightly, but significantly, from Eq. (51). This was the first demonstration that the eigenvalue repulsion in a disordered wire could not be precisely logarithmic (Beenakker, 1993a).

The variance $\operatorname{Var} A$ is the second cumulant of the distribution function $P(A)$. What about higher-order cumulants? Politzer (1989) has shown that the cumulants of order three and higher of a linear statistic $A$ vanish in the large- $N$ limit. This means that $P(A)$ tends to a Gaussian distribution in that limit. Politzer's argument is that the linearity of the relation (47) between $\rho$ and $V$ implies that for each $p \geqslant 3$ the functional derivative $\delta^{p-1} \rho / \delta V^{p-1}$ vanishes, and hence that the $p$-point correlation function as well as the $p$ th cumulant vanish. Only the two-point correlation function (proportional to $\delta \rho / \delta V$ ) and the second cumulant survive the large- $N$ limit.

\section{E. Overview}

The two questions that the random-matrix theory of quantum transport addresses are the following. What is the ensemble of scattering matrices? How to obtain from it the statistics of transport properties? In this article we review the answer to both questions for the two geometries where the answer is known: a quantum dot and a disordered wire.

The quantum dot is the easiest of the two geometries. For the first question we rely on Efetov's demonstration that the Hamiltonian of a disordered metal grain is distributed according to the Wigner-Dyson ensemble (1). The corresponding distribution of scattering matrices follows upon coupling the bound states inside the grain to propagating modes outside. If the coupling is via quantum point contacts, the scattering matrix is distributed according to the circular ensemble. (A quantum point contact is a narrow opening, much smaller than the mean free path, with a quantized conductance of $N G_{0}$.) The circular ensemble is defined by

$$
P(S)=\text { constant, }
$$

that is to say, the scattering matrix $S$ is uniformly distributed in the unitary group, subject only to the constraints imposed by time-reversal and/or spin-rotation symmetry. The corresponding distribution of the transmission eigenvalues is of the form of Eq. (40), with $f\left(T_{n}\right) \equiv 0$. Hence the eigenvalue repulsion in a quantum dot is logarithmic. Correlation functions of the transmission eigenvalues can be computed either by exact integration over the unitary group (which is practical for small $N$ ), or using the large- $N$ method of Sec. I.D.1. In particular, the limit $N \rightarrow \infty$ of the variance of a linear statistic is given by Eq. (61). The circular ensemble does not say how the scattering matrices at different energies or magnetic-field values are correlated. For that information one needs to return to the underlying Hamiltonian ensemble.

Historically, the latter approach came first: Verbaarschot, Weidenmüller, and Zirnbauer (1985), and Iida, Weidenmüller, and Zuk (1990a, 1990b) computed correlators of scattering matrix elements and moments of the conductance directly from the Hamiltonian ensemble. More recently, the entire distribution function of the transmission eigenvalues was determined starting from the ensemble of scattering matrices (Baranger and Mello, 1994; Jalabert, Pichard, and Beenakker, 1994). The equivalence of the two approaches has been established by Brouwer (1995). Both random-matrix ap- 


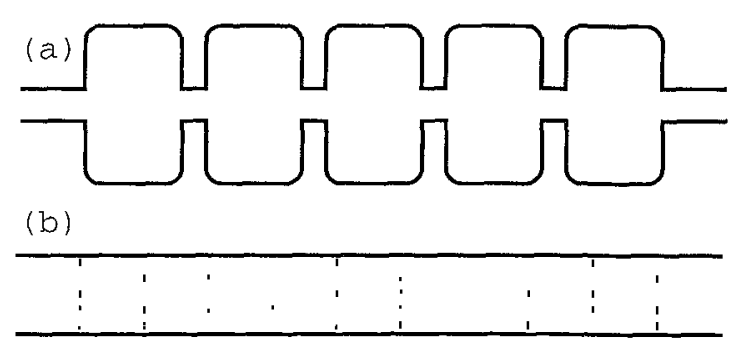

FIG. 5. Two ways to construct a conductor with the geometry of a wire: (a) Strongly scattering cavities, coupled in series via ideal leads, (b) weakly disordered segments in series. On long length scales, the two geometries have equivalent statistical properties. The number of scattering channels $N$ is determined by the width of the ideal leads in case (a) and by the width of the disordered segments in case (b).

proaches agree with Efetov's $(1982,1983)$ supersymmetric field theory of a disordered metal grain. There is considerable numerical and analytical evidence that they apply generically to any chaotic cavity, regardless of whether the chaos is due to impurity or boundary scattering (Bohigas, Giannoni, and Schmit, 1984; Andreev et al., 1996).

Once transport through a single quantum dot is understood, a logical next step is to connect many quantum dots in series, so that they form a wire [Fig. 5(a)]. Iida, Weidenmüller, and Zuk (1990a, 1990b), Weidenmüller (1990) and Altland (1991) computed the mean and variance of the conductance for such a model. An altogether different approach was taken earlier by Dorokhov (1982) and by Mello, Pereyra, and Kumar (1988). The wire is divided into weakly scattering segments (short compared to the mean free path $l$ ), so that the effect of adding a new segment can be determined by perturbation theory [Fig. 5(b)]. The result is a differential equation for the evolution with increasing wire length $L$ of the distribution function of the variables $\lambda_{n}=\left(1-T_{n}\right) / T_{n}$ :

$$
\begin{aligned}
l \frac{\partial}{\partial L} & P\left(\lambda_{1}, \lambda_{2}, \ldots, \lambda_{N}, L\right) \\
\quad= & \frac{2}{\beta N+2-\beta} \sum_{n=1}^{N} \frac{\partial}{\partial \lambda_{n}} \lambda_{n}\left(1+\lambda_{n}\right) J \frac{\partial}{\partial \lambda_{n}} \frac{P}{J} .
\end{aligned}
$$

The Jacobian

$$
J\left(\left\{\lambda_{n}\right\}\right)=\prod_{l<J}\left|\lambda_{l}-\lambda_{J}\right|^{\beta}
$$

relates volume elements in the polar decomposition (28) of the transfer matrix,

$$
d \mu(M)=J \prod_{\alpha} d \mu\left(U_{\alpha}\right) \prod_{l} d \lambda_{l} .
$$

The evolution equation (64) is known as the DorokhovMello-Pereyra-Kumar (DMPK) equation. For some time it was believed that the solution to Eq. (64) was of the form of Eq. (42). The exact solution (Beenakker and Rejaei, 1993, 1994a) of the DMPK equation for $\beta=2$

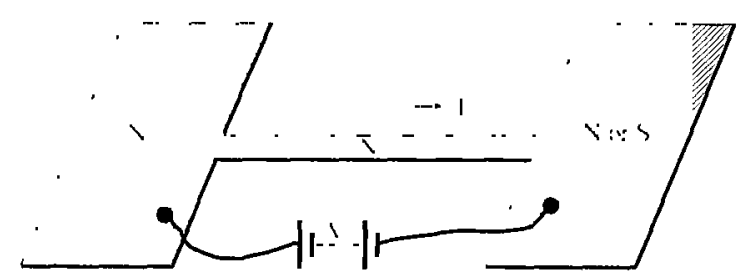

FIG. 6. A current $I$ is passed through a conductor by connecting it to two electron reservoirs (shaded) at a voltage difference $V$. The conductor and one of the two reservoirs are normal metals $(\mathrm{N})$, while the other reservoir may be in the superconducting state (S).

showed that this is not the case, and that the eigenvalue repulsion implied by Eq. (64) is not logarithmic, as it is in Eq. (42).

The model of quantum dots in series of Iida, Weidenmüller, and Zuk (1990a, 1990b) reduces on large length scales to a supersymmetric field theory known as the one-dimensional nonlinear $\sigma$ model (Mirlin, MüllerGroeling, and Zirnbauer, 1994). This model was originally derived by Efetov and Larkin (1983), starting from a Hamiltonian with randomly distributed impurities. A later derivation, due to Fyodorov and Mirlin (1991, 1994), uses a banded random matrix to model the Hamiltonian of the disordered wire. The DMPK equation and the $\sigma$ model of one-dimensional localization originated almost simultaneously in the early eighties, and at the same institute (Dorokhov, 1982, 1983; Efetov and Larkin, 1983). Nevertheless, work on both approaches proceeded independently over the next decade. The equivalence of the DMPK equation and the $\sigma$ model was finally demonstrated in 1996, by Brouwer and Frahm. This review is based on the DMPK equation. The $\sigma$ model is reviewed extensively in a monograph by Efetov (1996).

In order to study electronic transport through a quantum dot or a disordered wire, it has to be connected to two electron reservoirs (see Fig. 6). A current is passed through the system by bringing the reservoirs out of equilibrium. In Secs. II and III we assume that both reservoirs are in the normal state. In Sec. IV we consider the case that one of the two reservoirs is a superconductor. At the interface between the normal metal and the superconductor a peculiar scattering process occurs, discovered in 1964 by Andreev. This scattering process, known as Andreev reflection, converts dissipative current in the normal metal into dissipationless supercurrent in the superconductor. Andreev reflection modifies the quantum-interference effects existing in the normal state and introduces new effects as well. Random-matrix theory is particularly suited to contrast the two cases of normal and superconducting reservoirs, because the same scattering-matrix ensembles can be used. [For reviews devoted solely to normal-metal-superconductor junctions, see Klapwijk (1994), Beenakker (1995), and Van Wees and Takayanagi (1997).] If both reservoirs are superconductors, then the system is a Josephson junction, which supports a current in equilibrium. This 
current is a thermodynamic, rather than a (nonequilibrium) transport property, and will not be considered here. [For a review of the scattering-matrix approach to the theory of the Josephson effect, see Beenakker (1992b).]

This review was written in an attempt to provide a complete coverage of the present status of the randommatrix theory of quantum transport. Mindful of my own limitations, I apologize to those whose works I have overlooked or not sufficiently appreciated. No attempt was made to include other theories of transport, nor random-matrix theories of other than transport properties. Moreover, the adjective "quantum" is meant to exclude classical waves. Many of the effects described here have optical analogues that can be studied by the same random-matrix techniques. This provides an interesting opportunity for future research, which we will touch on in Sec. V.

\section{QUANTUM DOTS}

A cavity of submicron dimensions, etched in a semiconductor, is called a quantum dot. Quantummechanical phase coherence strongly affects its electronic properties, hence the adjective quantum. We consider the generic case that the classical motion in the cavity can be regarded as chaotic on time scales long compared to the ergodic time $\tau_{\text {ergodic. As discussed in }}$ Sec. I.B, the Hamiltonian of this closed system is then distributed according to the Wigner-Dyson ensemble, on energy scales small compared to the Thouless energy $E_{\text {c,closed }} \simeq \hbar / \tau_{\text {ergodic }}$. In order of magnitude, $E_{\text {c,closed }} \simeq\left(\hbar v_{\mathrm{F}} / L^{2}\right)$ min $(l, L)$ in a cavity of linear dimension $L$, mean free path $l$, and Fermi velocity $v_{\mathrm{F}}$. It does not matter for Wigner-Dyson statistics whether motion inside the cavity is ballistic $(L \ll l)$ or diffusive $(L \gg l)$. The material inside the quantum dot is assumed to be a good metal, which means that $E_{\mathrm{c}, \text { closed }}$ should be much greater than the mean level spacing $\delta$. The Fermi wavelength $\lambda_{\mathrm{F}}$ in a good metal is much smaller than $l$, so that the wave functions are extended rather than localized.

The transport properties of the quantum dot can be measured by coupling it to two electron reservoirs and bringing them out of equilibrium. This open system can still be regarded as chaotic, if the coupling is sufficiently weak that the mean dwell time ${ }^{10} \tau_{\text {dwell }}$ of an electron exceeds $\tau_{\text {ergodic }}$. In terms of energies, this condition can be written as $E_{\text {c,open }} \ll E_{\text {c,closed }}$, where $E_{\text {c,open }} \simeq \hbar / \tau_{\text {dwell }}$ is the Thouless energy of the open system. The ratio $E_{\text {c,open }} / \delta$ is of the order of the conductance $G$ of the quantum dot in units of $e^{2} / h$. While we do require $\delta \ll E_{\text {c,closed }}$, we do not restrict the relative magnitude of $\delta$ and $E_{\text {c,open }}$. Under the condition $E_{\text {c,open }} \ll E_{\text {c,closed }}$,

\footnotetext{
${ }^{10}$ The mean dwell time in a chaotic cavity is given by $2 \pi \hbar / \tau_{\text {dwell }}=\delta \Sigma_{n} \Gamma_{n}$, where $\Gamma_{n}$ is the tunnel probability of mode $n$ through a point contact. For example, in the case of two ballistic point contacts containing $N_{1}, N_{2}$ modes, one has $2 \pi \hbar / \tau_{\text {dwell }}=\left(N_{1}+N_{2}\right) \delta$.
}

transport quantities are insensitive to microscopic properties of the quantum dot, such as the shape of the cavity and the degree of disorder. In particular, just as in the closed system, it does not matter whether the motion is ballistic or diffusive inside the cavity. This universality does not extend to the contacts: it matters whether the coupling to the reservoirs is via ballistic point contacts or via tunnel barriers. We will see that the distribution of the scattering matrix is given by the circular ensemble for ballistic contacts and the Poisson kernel for tunneling contacts.

Throughout most of this section we will assume noninteracting electrons. This is justified if capacitive charging of the quantum dot relative to the reservoirs is insignificant, which it is if the coupling is via ballistic point contacts, but usually not if the coupling is via tunnel barriers.

\section{A. Transport theory of a chaotic cavity}

A random-matrix theory of transport through a chaotic cavity can be based either on an ensemble of scattering matrices or on an ensemble of Hamiltonians. We introduce these two approaches separately and then discuss their relationship and microscopic justification.

\section{Circular ensemble of scattering matrices}

Blümel and Smilansky (1990) found that the correlations of the phase shifts $\phi_{n}$ for chaotic scattering are well described by the distribution function

$$
P\left(\left\{\phi_{n}\right\}\right) \propto \prod_{n<m}\left|\exp \left(i \phi_{n}\right)-\exp \left(i \phi_{m}\right)\right|^{\beta}
$$

of the circular ensemble (for a review, see Smilansky, 1990). The circular ensemble was introduced by Dyson (1962a) as a mathematically more tractable alternative to the Gaussian ensemble. Baranger and Mello (1994) and Jalabert, Pichard, and Beenakker (1994) based a transport theory on the circular ensemble. For this purpose one needs to know the statistics of the transmission eigenvalues $T_{n}$, which are not directly related to the scattering phase shifts $\phi_{n}$. (The relationship involves both the eigenvalues and the eigenfunctions of the scattering matrix.)

The calculation of $P\left(\left\{T_{n}\right\}\right)$ starts from the defining property of the circular ensemble, that is that the scattering matrix $S$ is uniformly distributed in the unitary group, subject only to the symmetry and self-duality constraints imposed by time-reversal and spin-rotation symmetry (see Sec. I.C.1). Uniformity is defined with respect to a measure $d \mu(S)$ that is invariant under multiplication: $d \mu(S)=d \mu(U S V)$ for arbitrary unitary matrices $U, V$ such that the product $U S V$ still satisfies the constraints imposed on $S$. (This requires $V=U^{\mathrm{T}}$ for $\beta=1$ and $V=U^{\mathrm{R}}$ for $\beta=4$.) This measure is known as the "invariant measure" or "Haar measure" (Hamermesh, 1962). The probability distribution in the circular ensemble is thus given by 


$$
P\left(\left\{x_{n}\right\}\right) \prod_{l} d x_{l}=\frac{1}{\mathcal{V}} d \mu(S),
$$

where $\mathcal{V}=\int d \mu(S)$ is the volume of the matrix space and $\left\{x_{n}\right\}$ is a set of independent variables that parametrizes $S$. The general method to compute the invariant measure in a given parametrization is to consider the change $d S$ associated with an infinitesimal change $d x_{n}$ in the $x_{n}$ 's. The invariant arclength $\operatorname{Tr} d S d S^{\dagger}$ defines the metric tensor $g_{l j}$ according to

$$
\operatorname{Tr} d S d S^{\dagger}=\sum_{l, j} g_{l j} d x_{t} d x_{l} .
$$

The determinant Det $g$ then yields the invariant measure

$$
d \mu(S)=|\operatorname{Det} g|^{1 / 2} \prod_{l} d x_{l}
$$

and hence the distribution $P\left(\left\{x_{n}\right\}\right) \propto \mid$ Det $\left.g\right|^{1 / 2}$.

In the scattering phase-shift representation the measure takes the form (Dyson, 1962a)

$$
d \mu(S)=\prod_{n<m}\left|\exp \left(i \phi_{n}\right)-\exp \left(i \phi_{m}\right)\right|^{\beta} d \mu(U) \prod_{l} d \phi_{l},
$$

where $U$ is the matrix of eigenvectors that diagonalizes the scattering matrix: $\left(U^{-1} S U\right)_{n m}=\delta_{n m} \exp \left(i \phi_{n}\right)$. The matrix $U$ is orthogonal, unitary, or symplectic, for $\beta=1,2$, or 4 , respectively. [If $\beta=4$ each eigenvalue is twofold degenerate, and the products in Eq. (71) include only the distinct eigenvalues.] The invariant measure (71) implies that the eigenvectors and eigenvalues of $S$ are distributed independently. The matrix of eigenvectors $U$ is uniformly distributed in the orthogonal, unitary, or symplectic group. The eigenvalues $\exp \left(i \phi_{n}\right)$ are distributed according to Eq. (67). This ensemble is called "circular" because the eigenvalue density is constant on the unit circle in the complex plane. The adjective orthogonal, unitary, or symplectic is added to distinguish the cases $\beta=1,2$, or 4 . Note that this name derives from the matrix of eigenvectors $U$, not from the scattering matrix $S$ (see Table II). For example, the circular orthogonal ensemble for $\beta=1$ (COE) is the ensemble of uniformly distributed, unitary symmetric matrices. The circular symplectic ensemble (CSE, $\beta=4$ ) contains the unitary self-dual matrices, and the circular unitary ensemble (CUE, $\beta=2$ ) contains all unitary matrices.

It is sometimes useful to be able to write averages over the COE and CSE as averages over the CUE. ${ }^{11}$ For the COE, consisting of unitary symmetric matrices, this is achieved by the representation $S=U U^{\mathrm{T}}$. Averaging $S$ over the COE is then equivalent to averaging $U$ over the CUE:

\footnotetext{
${ }^{11}$ There also exists a relationship between averages over the COE and CSE, which is described by Brouwer and Beenakker (1996a).
}

$$
\langle f(S)\rangle_{S \in \mathrm{COE}}=\left\langle f\left(U U^{\mathrm{T}}\right)\right\rangle_{U \in \mathrm{CUE}} .
$$

For the CSE one first needs to represent the $N \times N$ quaternion matrix $S$ by a $2 N \times 2 N$ complex matrix $U$. We denote this representation by $S \cong U$. The dual of $S$ is $S^{\mathrm{R}} \cong \mathcal{C}^{\mathrm{T}} U^{\mathrm{T}} \mathcal{C}$, where $\mathcal{C}$ is a $2 N \times 2 N$ matrix with zero elements, except for $\mathcal{C}_{2 \imath-1,2 t}=1, \quad \mathcal{C}_{2 \imath, 2 l-1}=-1$ $(i=1,2, \ldots, N)$ :

$$
\mathcal{C}=\left(\begin{array}{cccccccc}
0 & 1 & & & & & & \\
-1 & 0 & 0 & & & & & \\
& 0 & 0 & 1 & & & & \\
& & -1 & 0 & 0 & & & \\
& & & \cdot & \cdot & . & & \\
& & & & \cdot & . & . & \\
& & & & & 0 & 0 & 1 \\
& & & & & & -1 & 0
\end{array}\right) .
$$

Note that $\mathcal{C}^{\mathrm{T}}=-\mathcal{C}$ and $\mathcal{C}^{2}=-1$. A self-dual matrix is represented by $S \cong U \mathcal{C}^{\mathrm{T}} U^{\mathrm{T}} \mathcal{C}$. Averaging $S$ over the CSE is equivalent to averaging $U$ over the CUE:

$$
\langle f(S)\rangle_{S \in \mathrm{CSE}}=\left\langle f\left(U \mathcal{C}^{\mathrm{T}} U^{\mathrm{T}} \mathcal{C}\right)\right\rangle_{U \in \mathrm{CUE}} .
$$

Averages over the CUE amount to an integration over the unitary group. A few integration formulas which we will need are collected in Appendix B.

The representation of $S$ in terms of the set of transmission eigenvalues $\left\{T_{n}\right\}$ is the polar decomposition (24). The corresponding measure is (Baranger and Mello, 1994; Jalabert, Pichard, and Beenakker, 1994)

$$
d \mu(S)=\prod_{n<m}\left|T_{n}-T_{m}\right|^{\beta} \prod_{k} T_{k}^{-1+\beta / 2} \prod_{\alpha} d \mu\left(U_{\alpha}\right) \prod_{l} d T_{\imath},
$$

where $\left\{U_{\alpha}\right\}$ is the set of independent unitary matrices in Eq. (24). The polar decomposition (24) assumes that the two leads attached to the cavity support the same number of transverse modes, so that the transmission matrices $t$ and $t^{\prime}$ are square matrices. More generally, one can consider the case that the number of modes $N_{1}$ and $N_{2}$ in the two leads is different, so that $t$ and $t^{\prime}$ are rectangular matrices. The two matrix products $t t^{\dagger}$ and $t^{\prime} t^{\prime \dagger}$ contain a common set of $\min \left(N_{1}, N_{2}\right)$ nonzero transmission eigenvalues. Only these appear in the invariant measure, which in comparison with Eq. (75) contains an extra factor $\Pi_{k} T_{k}^{(1 / 2) \beta\left|N_{2}-N_{1}\right|}$ in the exponent (Brouwer, 1994). The resulting probability distribution of the transmission eigenvalues is

$$
P\left(\left\{T_{n}\right\}\right) \propto \prod_{n<m}\left|T_{n}-T_{m}\right|^{\beta} \prod_{k} T_{k}^{(1 / 2) \beta\left(\left|N_{2}-N_{1}\right|+1-2 / \beta\right)} .
$$

\section{Poisson kernel}

The circular ensemble is the "most random" ensemble of scattering matrices. It would seem a natural choice for a chaotic cavity, which one could call the "most random" conductor. As we will see in Sec. II.A.4, 
this choice has a justification from microscopic theory. The notion of a "most random" ensemble can be made quantitative by associating an information entropy $\mathcal{S}=-\int d \mu(S) P(S) \ln P(S)$ with the probability distribution $P(S)$. The most random ensemble is then the ensemble which maximizes $\mathcal{S}$, subject to certain constraints (Balian, 1968). For the circular ensemble the only constraints are the symmetry $(\beta=1)$ or self-duality $(\beta=4)$ of $S$. Mello, Pereyra, and Seligman (1985), and more recently Baranger and Mello (1996a), have considered the additional set of constraints

$$
\int d \mu(S) S^{p} P(S)=\bar{S}^{p}, \quad p=1,2, \ldots
$$

where $\bar{S}$ is a given subunitary matrix. (Subunitary means that the eigenvalues of $\bar{S} \bar{S}^{\dagger}$ are $\leqslant 1$.) The distribution which maximizes the entropy subject to these constraints is

$$
P(S) \propto\left|\operatorname{Det}\left(1-\bar{S}^{\dagger} S\right)\right|^{-\beta\left(N_{1}+N_{2}-1+2 / \beta\right)} .
$$

The circular ensemble is the special case $\bar{S}=0$, so that $P(S)=$ constant. Equation (78) generalizes the circular ensemble to nonzero-average scattering matrix $\bar{S}$.

The distribution (78) is known in the mathematical literature as the Poisson kernel (Hua, 1963). It was introduced into random-matrix theory by Krieger (1965) and first applied to a chaotic cavity by Doron and Smilansky (1992). The name originates from the problem of determining the analytic function $V(\bar{S})$ of subunitary matrices $\bar{S}$, from the knowledge of $V(S)$ for unitary $S$. This problem is the multidimensional generalization of the two-dimensional electrostatic problem of computing the potential inside a cylinder from the values it takes on the surface. The solution $f(\bar{S})=\int d \mu(S) f(S) P(S)$ is called Poisson's formula in the electrostatic context. Equation (77) is known as the analyticity-ergodicity constraint (Mello, 1995). The name refers to the analyticity requirement that $S$ has poles only in the lower half of the complex-energy plane and to the ergodicity assumption that ensemble averages equal spectral averages. Together, these two conditions imply $\left\langle S^{p}\right\rangle=\langle S\rangle^{p}$ (with $p$ a positive integer), so that a single matrix $\bar{S}$ determines all positive moments of $S$.

The circular ensemble is appropriate for a chaotic cavity that is coupled to the leads by means of ballistic point contacts ("ideal" leads), since the only property of the coupling which enters is the number of modes $N_{1}, N_{2}$. More generally, one can consider nonideal leads, containing tunnel barriers (see Fig. 7). Assume that the segment of the lead between the tunnel barrier and the cavity is long enough, so that the scattering matrices $S_{1}, S_{2}$ of barriers 1 and 2, as well as the scattering matrix $S_{0}$ of the cavity, are well defined. The scattering matrix $S$ of the whole structure is obtained by multiplying the three transfer matrices corresponding to $S_{1}, S_{0}$, and $S_{2}$. Brouwer (1995) has shown that if $S_{0}$ is distributed according to the circular ensemble, then $S$ is distributed according to the Poisson kernel. The average scattering matrix in this case is

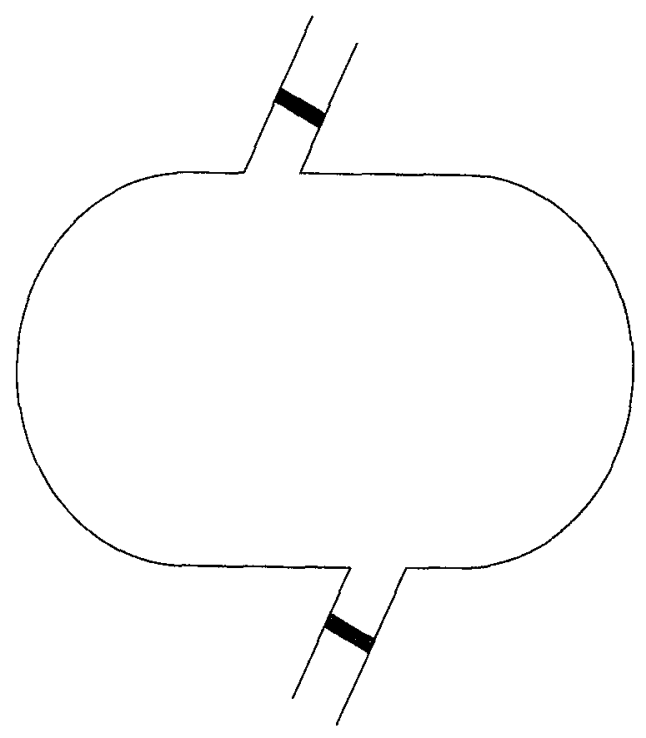

FIG. 7. Chaotic cavity (the stadium billiard) coupled to two reservoirs via narrow leads containing tunnel barriers. The distribution of the scattering matrix is given by the Poisson kernel (78), which reduces to the circular ensemble in the absence of tunnel barriers in the leads.

$$
\bar{S}=\left(\begin{array}{cc}
r_{1} & 0 \\
0 & r_{2}
\end{array}\right)
$$

with $r_{1}$ and $r_{2}$ the reflection matrices of barriers 1 and 2 for electrons incident from the reservoirs. The eigenvalues of $1-\bar{S} \bar{S}^{\dagger}$ are the tunnel probabilities $\Gamma_{n}$ through the leads.

\section{Gaussian ensemble of Hamiltonians}

The Hamiltonian approach to transport through a chaotic cavity goes back to work in the sixties on nuclear reactions (Mahaux and Weidenmüller, 1969). The Hamiltonian of the cavity connected to leads by tunnel barriers is represented by

$$
\begin{aligned}
H= & \sum_{a}|a\rangle E_{\mathrm{F}}\left\langle a\left|+\sum_{\mu, \nu}\right| \mu\right\rangle \mathcal{H}_{\mu \nu}\langle\nu| \\
& +\sum_{\mu, a}\left(|\mu\rangle W_{\mu a}\langle a|+| a\rangle W_{\mu a}^{*}\langle\mu|\right) .
\end{aligned}
$$

The set $\{|a\rangle\}\left(a=1,2, \ldots, N\right.$, with $N=N_{1}+N_{2}$ the total number of propagating modes in the leads) forms a basis of scattering states in the leads at the Fermi energy $E_{\mathrm{F}}$. The set of bound states in the isolated cavity is denoted by $\{|\mu\rangle\}(\mu=1,2, \ldots, M)$. The finite number $M$ is artificial and will eventually be taken to infinity. The matrix elements $\mathcal{H}_{\mu \nu}$ form a Hermitian $M \times M$ matrix $\mathcal{H}$, with real $(\beta=1)$, complex $(\beta=2)$, or real quaternion $(\beta=4)$ elements. The coupling constants $W_{\mu a}$ form a real (complex, real quaternion) $M \times N$ matrix $W$, which is assumed to be independent of energy. The $N \times N$ scat 
tering matrix $S$ associated with the Hamiltonian $H$ equals $^{12}$

$$
\begin{aligned}
S & =1-2 \pi i W^{\dagger}\left(E_{\mathrm{F}}-\mathcal{H}+i \pi W W^{\dagger}\right)^{-1} W \\
& =\frac{1+i \pi W^{\dagger}\left(\mathcal{H}-E_{\mathrm{F}}\right)^{-1} W}{1-i \pi W^{\dagger}\left(\mathcal{H}-E_{\mathrm{F}}\right)^{-1} W} .
\end{aligned}
$$

One verifies that, for $\beta=1,2$, and 4 , the matrix $S$ is unitary symmetric, unitary, and unitary self-dual, respectively.

The Hamiltonian $\mathcal{H}$ of a chaotic cavity is distributed according to the Gaussian ensemble,

$$
P(\mathcal{H}) \propto \exp \left[-\beta(\pi / 2 \delta)^{2} M^{-1} \operatorname{Tr} \mathcal{H}^{2}\right] .
$$

The coefficient $\delta$ equals the mean level spacing at the Fermi level in the limit $M \rightarrow \infty$ (Mehta, 1991). The average scattering matrix $\bar{S}$ in this limit is given by (Verbaarschot, Weidenmüller, and Zirnbauer, 1985)

$$
\bar{S}=\frac{M \delta-\pi^{2} W^{\dagger} W}{M \delta+\pi^{2} W^{\dagger} W} .
$$

Comparison with Eq. (79) shows that the eigenvalue $w_{n}$ of the coupling-matrix product $W^{\dagger} W$ is related to the tunnel probability $\Gamma_{n}$ of mode $n$ in the lead by

$$
\begin{aligned}
\Gamma_{n} & =\frac{4 M \delta \pi^{2} w_{n}}{\left(M \delta+\pi^{2} w_{n}\right)^{2}}, \\
w_{n} & =\frac{M \delta}{\pi^{2} \Gamma_{n}}\left(2-\Gamma_{n} \pm 2 \sqrt{1-\Gamma_{n}}\right) .
\end{aligned}
$$

Notice that $\Gamma_{n}$ does not determine $w_{n}$ uniquely.

The approach of coupling $M$ eigenstates of the cavity to $N$ scattering channels in the leads introduces a large number of coupling constants $W_{\mu a}$, while a much smaller number of parameters $\Gamma_{n}$ determine the transport properties at the Fermi level. This is why the scattering-matrix approach is more convenient than the Hamiltonian approach in cases that the energy dependence of the transport properties is not required. The equivalence of the two approaches is discussed in the next subsection, together with the microscopic justification.

\section{Justification from microscopic theory}

A microscopic justification for the Gaussian ensemble has been provided by Efetov $(1982,1983)$ for a disordered metal grain, and by Andreev et al. (1996) for a chaotic billiard (see Sec. I.B.2). A microscopic justification for the circular ensemble and the Poisson kernel has been provided indirectly by a demonstration of the

\footnotetext{
${ }^{12}$ The Hamiltonian represented by Eq. (80) gives $S=1$ in the case $W=0$ of an isolated cavity. A more general Hamiltonian would give $S=S_{0}$, with $S_{0}$ a "background" scattering matrix that does not couple to the cavity (Nishioka and Weidenmüller, 1985). This more general case amounts to the transformation $S \rightarrow U S V$, where the unitary matrices $U$ and $V$ are independent of $\mathcal{H}$.
}

equivalence with the Gaussian ensemble (Brouwer, 1995; see also Lewenkopf and Weidenmüller, 1991). We present an outline of Brouwer's equivalence proof. It proceeds in two steps. The first step is to show that, in the limit $M \rightarrow \infty$, the Gaussian distribution (82) can be replaced by the Lorentzian distribution

$$
P(\mathcal{H}) \propto \operatorname{Det}\left[(M \delta / \pi)^{2}+\mathcal{H}^{2}\right]^{-(\beta M+2-\beta) / 2} .
$$

The second step is to show that, for any $M \geqslant N$, the distribution $P(S)$ of the scattering matrix obtained from Eq. (85) is the Poisson kernel (78).

The replacement of Eq. (82) by Eq. (85) is allowed because the eigenvector and eigenvalue distributions of the Gaussian and the Lorentzian ensemble are equal on a fixed energy scale, in the limit $M \rightarrow \infty$. The equivalence of the eigenvector distributions is obvious: the distribution of $\mathcal{H}$ depends solely on the eigenvalues for both the Lorentzian and the Gaussian ensemble, so that the eigenvector distribution is uniform for both ensembles. The equivalence of the distribution of the eigenvalues is proven by an explicit comparison of the $p$-point correlation functions. (These can be computed exactly in both the Gaussian and the Lorentzian ensembles, using the method of orthogonal polynomials.)

The technical reason for working with the Lorentzian ensemble instead of the Gaussian ensemble is the invariance property that, if $\mathcal{H}$ has a Lorentzian distribution, then its inverse $\mathcal{H}^{-1}$ as well as any submatrix of $\mathcal{H}$ have a Lorentzian distribution. This property makes it particularly easy to compute the distribution of the scattering matrix, for any $M \geqslant N$. The resulting distribution has the form of a Poisson kernel,

$$
\begin{aligned}
& P(S) \propto\left|\operatorname{Det}\left(1-\bar{S}^{\dagger} S\right)\right|^{-\beta N-2+\beta}, \\
& \bar{S}=\frac{M \delta-i \pi E_{\mathrm{F}}-\pi^{2} W^{\dagger} W}{M \delta-i \pi E_{\mathrm{F}}+\pi^{2} W^{\dagger} W} .
\end{aligned}
$$

Equation (83) for the average scattering matrix is recovered from Eq. (87) in the limit $M \rightarrow \infty$.

The conclusion is that the Poisson kernel for the distribution of scattering matrices (and in particular the circular ensemble, to which the Poisson kernel reduces for $\bar{S}=0$ ) is equivalent to the Lorentzian ensemble of Hamiltonians for any $M \geqslant N$. The Lorentzian ensemble, in turn, is equivalent in the limit $M \rightarrow \infty$ to the Gaussian ensemble, which for a chaotic cavity has been derived from microscopic theory. This provides the microscopic justification for the random-matrix theory of transport through a quantum dot.

\section{B. Weak localization}

Consider a chaotic cavity with two small holes of the same size. An electron which is injected through one of the holes will exit either through the same hole (reflection) or through the other hole (transmission). Classically, chaotic motion in the cavity implies that the transmission and reflection probabilities are equal. Quantum mechanically, the transmission probability is slightly smaller than the reflection probability. This effect is 
known as "weak localization," after the analogous effect in disordered metals. In a semiclassical formulation, the enhancement of the reflection probability is due to the constructive interference of pairs of time-reversed trajectories (Baranger, Jalabert, and Stone, 1993a, 1993b; Argaman, 1995, 1996; Aleiner and Larkin, 1997). A magnetic field breaks the time-reversal symmetry, thereby destroying the constructive interference and equalizing the transmission and reflection probabilities. The magnitude of the weak-localization effect in a quantum dot was first computed by Iida, Weidenmüller, and Zuk (1990a, 1990b), using the Hamiltonian approach described in Sec. II.A.3. The calculation is easier using the scattering-matrix approach of Sec. II.A.1 (Baranger and Mello, 1994; Jalabert, Pichard, and Beenakker, 1994). Using the latter approach, we discuss the weaklocalization correction to the conductance and the generalization to other transport properties.

\section{Conductance}

In the absence of time-reversal symmetry, the scattering matrix $S$ of a chaotic cavity is uniformly distributed over the unitary group. This is the circular unitary ensemble (CUE, $\beta=2)$. The average of the scattering probability $\left|S_{n m}\right|^{2}$ follows from Eq. (B4),

$$
\left\langle\left|S_{n m}\right|^{2}\right\rangle_{\mathrm{CUE}}=\int d \mu(S) S_{n m} S_{n m}^{*}=\frac{1}{N_{1}+N_{2}} .
$$

[The integral is over $\mathcal{U}\left(N_{1}+N_{2}\right)$ with invariant measure $d \mu(S)$, normalized such that $\int d \mu(S)=1$, where $N_{1}$ and $N_{2}$ are the number of modes in the leads connected to contacts 1 and 2.] In the CUE, scattering between two different modes $(n \neq m)$ is equally probable as between two identical modes $(n=m)$. In the presence of timereversal symmetry, $S$ is unitary and symmetric (assuming no spin-orbit scattering). This is the circular orthogonal ensemble (COE, $\beta=1$ ). The average of $\left|S_{n m}\right|^{2}$ follows from Eqs. (72) and (B5),

$$
\begin{aligned}
\left\langle\left|S_{n m}\right|^{2}\right\rangle_{\mathrm{COE}} & =\int d \mu(U) \sum_{k, k^{\prime}=1}^{N_{1}+N_{2}} U_{n k} U_{m k} U_{n k^{\prime}}^{*} U_{m k^{\prime}}^{*} \\
& =\frac{1+\delta_{n m}}{N_{1}+N_{2}+1} .
\end{aligned}
$$

Scattering from mode $n$ back to mode $n$ is now twice as probable as from mode $n$ into another mode $m$. The absence of spin-orbit scattering is essential. In the circular symplectic ensemble (CSE, $\beta=4$ ) one obtains from Eqs. (74) and (B5) the average ${ }^{13}$

$$
\begin{aligned}
\left\langle\left|S_{n m}\right|^{2}\right\rangle_{\mathrm{CSE}}= & \frac{1}{2} \sum_{p, q=0}^{1} \int d \mu(U)\left(U \mathcal{C} U^{\mathrm{T}} \mathcal{C}\right)_{2 n-p, 2 m-q} \\
& \times\left(U^{*} \mathcal{C} U^{\dagger} \mathcal{C}\right)_{2 n-p, 2 m-q}
\end{aligned}
$$

\footnotetext{
${ }^{13}$ The absolute value $|Q|$ of a quaternion number $Q$ (represented by a $2 \times 2$ matrix) is defined by $|Q|^{2}=\frac{1}{2} \operatorname{Tr} Q Q^{\dagger}$.
}

$$
=\frac{2-\delta_{n m}}{2 N_{1}+2 N_{2}-1} .
$$

[The integration is over $\mathcal{U}\left(2 N_{1}+2 N_{2}\right)$.] Scattering between the same mode is now less probable than between different modes. Equations (88)-(90) can be summarized in one $\beta$-dependent expression,

$$
\left\langle\left|S_{n m}\right|^{2}\right\rangle=\frac{1-(1-2 / \beta) \delta_{n m}}{N_{1}+N_{2}-1+2 / \beta}
$$

According to the Landauer formula (33), the conductance $G$ of the quantum dot is obtained from the scattering probabilities $\left|S_{n m}\right|^{2}$ by summing $n$ over all $N_{1}$ modes in lead 1 and summing $m$ over all $N_{2}$ modes in lead 2,

$$
G=G_{0} \sum_{n=1}^{N_{1}} \sum_{m=N_{1}+1}^{N_{1}+N_{2}}\left|S_{n m}\right|^{2},
$$

where $G_{0}=2 e^{2} / h$. Substitution of Eq. (91) into Eq. (92) yields the average conductance (Baranger and Mello, 1994)

$$
\left\langle G / G_{0}\right\rangle=\frac{N_{1} N_{2}}{N_{1}+N_{2}-1+2 / \beta} .
$$

For $N_{1} \gg 1, N_{2} \gg 1$ we may expand

$$
\left\langle G / G_{0}\right\rangle=\frac{N_{1} N_{2}}{N_{1}+N_{2}}+\left(1-\frac{2}{\beta}\right) \frac{N_{1} N_{2}}{\left(N_{1}+N_{2}\right)^{2}} .
$$

The first term in Eq. (94) is the classical series conductance $G_{\text {series }}=G_{0}\left(N_{1}^{-1}+N_{2}^{-1}\right)^{-1}$ of the two contact conductances $N_{1} G_{0}$ and $N_{2} G_{0}$. The second term is the weak-localization correction $\delta G$. For the case of two identical contacts one has simply

$$
\delta G / G_{0}=\frac{1}{4}\left(1-\frac{2}{\beta}\right), \quad N_{1}=N_{2} \gg 1 .
$$

\section{Other transport properties}

To compute the weak-localization correction for other transport properties, one needs the density of the transmission eigenvalues. We use the parametrization $T_{n}=1 /\left(1+\lambda_{n}\right)$ and write the probability distribution (76) of the $N_{\min } \equiv \min \left(N_{1}, N_{2}\right)$ nonzero transmission eigenvalues in the form of a Gibbs distribution:

$$
\begin{aligned}
& P\left(\left\{\lambda_{n}\right\}\right) \propto \exp \left[\beta \sum_{i<j} \ln \left|\lambda_{i}-\lambda_{j}\right|-\beta \sum_{i} V\left(\lambda_{i}\right)\right], \\
& V(\lambda)=\frac{1}{2}\left(N_{1}+N_{2}-1+2 / \beta\right) \ln (1+\lambda) .
\end{aligned}
$$

The density $\rho(\lambda)=\left\langle\Sigma_{n} \delta\left(\lambda-\lambda_{n}\right)\right\rangle$ of the $\lambda$ 's is determined for $N_{\min } \gg 1$ by the integral equation (A1). We decompose $\rho=\rho_{0}+\delta \rho$ into a contribution $\rho_{0}$ of order $N_{\min }$ and a correction $\delta \rho$ of order unity. Similarly, we decompose the potential $V=V_{0}+\delta V$ into two terms: $V_{0}=\frac{1}{2}\left(N_{1}+N_{2}\right) \ln (1+\lambda), \delta V=-\frac{1}{2}(1-2 / \beta) \ln (1+\lambda)$.

The leading-order contribution $\rho_{0}$ satisfies

$$
\int_{0}^{\lambda_{c}} d \lambda^{\prime} \frac{\rho_{0}\left(\lambda^{\prime}\right)}{\lambda-\lambda^{\prime}}=\frac{d}{d \lambda} V_{0}(\lambda)
$$


where the singular integral is the principal value. [Equation (97) is the derivative with respect to $\lambda$ of Eq. (47).] The density $\rho_{0}$ vanishes for $\lambda \geqslant \lambda_{c}$. The general solution of this integral equation, with normalization $\int \rho_{0} d \lambda=N_{\min }$, is (Mikhlin, 1964)

$$
\begin{aligned}
\rho_{0}(\lambda)= & \pi^{-2}\left[\lambda\left(\lambda_{c}-\lambda\right)\right]^{-1 / 2}\left(\pi N_{\min }-\int_{0}^{\lambda_{c}} d \lambda^{\prime}\right. \\
& \left.\times \frac{\left[\lambda^{\prime}\left(\lambda_{c}-\lambda^{\prime}\right)\right]^{1 / 2}}{\lambda-\lambda^{\prime}} \frac{d}{d \lambda^{\prime}} V_{0}\left(\lambda^{\prime}\right)\right) .
\end{aligned}
$$

The free boundary $\lambda_{\mathrm{c}}$ is to be determined from $\rho_{0}\left(\lambda_{\mathrm{c}}\right)=0$. The resulting density is

$$
\begin{aligned}
& \rho_{0}(\lambda)=\frac{1}{\pi}\left(N_{1} N_{2}\right)^{1 / 2} \frac{1}{1+\lambda}\left(\frac{1}{\lambda}-\frac{1}{\lambda_{\mathrm{c}}}\right)^{1 / 2}, \\
& \lambda_{\mathrm{c}}=\frac{4 N_{1} N_{2}}{\left(N_{1}-N_{2}\right)^{2}},
\end{aligned}
$$

in agreement with a calculation using a different method (Nazarov, 1995a). For $N_{1}=N_{2}$ one may put $\lambda_{c} \rightarrow \infty$, and the density simplifies to (Baranger and Mello, 1994; Jalabert, Pichard, and Beenakker, 1994)

$$
\rho_{0}(\lambda)=\left(N_{1} / \pi\right)(1+\lambda)^{-1} \lambda^{-1 / 2} \text {. }
$$

Linearization of Eq. (A1) around $\rho_{0}$ yields an equation for $\delta \rho$,

$$
\begin{aligned}
\int_{0}^{\lambda_{c}} d \lambda^{\prime} \frac{\delta \rho\left(\lambda^{\prime}\right)}{\lambda-\lambda^{\prime}} & =\frac{d}{d \lambda} \delta V(\lambda)-\frac{1}{2}(1-2 / \beta) \frac{d}{d \lambda} \ln \rho_{0}(\lambda) \\
& =\frac{1}{4}(1-2 / \beta)\left[\lambda^{-1}-\left(\lambda-\lambda_{c}\right)^{-1}\right] .
\end{aligned}
$$

The solution is a delta-function peak at the two ends of the spectrum, with the same weight but opposite sign,

$$
\delta \rho(\lambda)=\frac{1}{4}(1-2 / \beta)\left[\delta\left(\lambda-0^{+}\right)-\delta\left(\lambda-\lambda_{\mathrm{c}}+0^{+}\right)\right] .
$$

Transforming back from $\lambda$ to $T$, one obtains the density of transmission eigenvalues $\rho(T)=\rho(\lambda) d \lambda / d T$. The average of a linear statistic $A=\Sigma_{n} a\left(T_{n}\right)$ then follows upon integration,

$$
\begin{aligned}
& \langle A\rangle=\frac{N_{1}+N_{2}}{2 \pi} \int_{T_{\mathrm{c}}}^{1} d T\left(\frac{T-T_{\mathrm{c}}}{1-T}\right)^{1 / 2} \frac{a(T)}{T}+\frac{1}{4}(1-2 / \beta) \\
& \quad \times\left[a(1)-a\left(T_{\mathrm{c}}\right)\right] \\
& T_{\mathrm{c}}=\left(\frac{N_{1}-N_{2}}{N_{1}+N_{2}}\right)^{2}
\end{aligned}
$$

One verifies that the result (94) for the conductance is recovered for $a(T)=T$. As an example of another transport property, we take the shot-noise power (35). Substitution of $a(T)=T(1-T)$ into Eq. (103) yields

$$
\left\langle P / P_{0}\right\rangle=\frac{\left(N_{1} N_{2}\right)^{2}}{\left(N_{1}+N_{2}\right)^{3}}-\left(1-\frac{2}{\beta}\right) \frac{N_{1} N_{2}\left(N_{1}-N_{2}\right)^{2}}{\left(N_{1}+N_{2}\right)^{4}} .
$$

The weak-localization correction vanishes if $N_{1}=N_{2}$ (Jalabert, Pichard, and Beenakker, 1994).

\section{Tunnel barriers}

In the presence of tunnel barriers in the leads, the distribution of the scattering matrix $S$ is given by the Poisson kernel (78). Equation (79) relates the ensembleaveraged scattering matrix $\bar{S}$ that appears in the Poisson kernel to the reflection matrices of the tunnel barriers. (The circular ensemble corresponds to $\bar{S}=0$.) The eigenvalue $\Gamma_{n}$ of $1-\bar{S} \bar{S}^{\dagger}$ is the tunnel probability of mode $n$ in the lead. The fluctuating part $\delta S \equiv S-\bar{S}$ of $S$ can be parametrized as

$$
\delta S=A(1-U B)^{-1} U C,
$$

where $U$ is a unitary matrix and the matrices $A, B$, and $C$ are such that the matrix

$$
S_{\text {barrier }}=\left(\begin{array}{ll}
\bar{S} & A \\
C & B
\end{array}\right)
$$

is unitary. In zero magnetic field one should require furthermore that $U$ and $S_{\text {barrier }}$ are symmetric (or self-dual in the presence of spin-orbit scattering). The usefulness of the parametrization (105) is that $U$ is distributed according to the circular ensemble, for any choice of $A$, $B$, and $C$ (Hua, 1963; Friedman and Mello, 1985a; Brouwer, 1995). Physically, $U$ corresponds to the scattering matrix of the cavity without the tunnel barriers in the leads, and $S_{\text {barricr }}$ corresponds to the scattering matrix of the tunnel barriers in the absence of the cavity.

The parametrization (105) reduces the problem of computing the average conductance to an integration of $U$ over the unitary group. The result of the integration will depend on $\bar{S}$, but not on $A, B$, or $C$. Because the conductance (92) is a rational function of $U$, the integration cannot be carried out in closed form. For $N \Gamma \gg 1$ a perturbative calculation is possible (Brouwer and Beenakker, 1996a). The result is

$$
\begin{aligned}
& \left\langle G / G_{0}\right\rangle=\frac{g_{1} g_{1}^{\prime}}{g_{1}+g_{1}^{\prime}}+\left(1-\frac{2}{\beta}\right) \frac{g_{2} g_{1}^{\prime 2}+g_{2}^{\prime} g_{1}^{2}}{\left(g_{1}+g_{1}^{\prime}\right)^{3}}, \\
& g_{p}=\sum_{n=1}^{N_{1}} \Gamma_{n}^{p}, \quad g_{p}^{\prime}=\sum_{n=N_{1}+1}^{N_{1}+N_{2}} \Gamma_{n}^{p} .
\end{aligned}
$$

The first term in Eq. (107) is the classical series conductance of the two tunnel conductances $G_{0} g_{1}$ and $G_{0} g_{1}^{\prime}$. The term proportional to $1-2 / \beta$ is the weak-localization correction. In the absence of tunnel barriers one has $g_{p}=N_{1}, g_{p}^{\prime}=N_{2}$, and Eq. (94) is recovered. In the case of two identical tunnel barriers $\left(N_{1}=N_{2}, \Gamma_{n}=\Gamma_{n+N_{1}}\right.$ for $n=1,2, \ldots, N_{1}$ ), Eq. (107) simplifies to (Iida, Weidenmüller, and Zuk, 1990a, 1990b)

$$
\left\langle G / G_{0}\right\rangle=\frac{g_{1}}{2}+\left(1-\frac{2}{\beta}\right) \frac{g_{2}}{4 g_{1}} .
$$

If all $\Gamma_{n}$ 's are equal to $\Gamma$, Eq. (109) simplifies further to $\left\langle G / G_{0}\right\rangle=\frac{1}{2} N_{1} \Gamma+\frac{1}{4}(1-2 / \beta) \Gamma$.

\section{Magnetoconductance}

A weak magnetic field suppresses the weaklocalization correction to the average conductance. In 
the absence (presence) of spin-orbit scattering, the magnetoconductance consists of a dip (peak) around $B=0$ of magnitude $\delta G \approx e^{2} / h$ and width $B_{\mathrm{c}}$. The flux $\Phi_{\mathrm{c}}$ through the particle at magnetic field $B_{\mathrm{c}}$ is of order $\Phi_{\mathrm{c}} \simeq(h / e)\left(E_{\mathrm{c}, \text { open }} / E_{\mathrm{c}, \text { closed }}\right)^{1 / 2}$. This is the flux at which time-reversal symmetry is broken on the energy scale $E_{\text {c,open }}$ (see Sec. I.B.3). Up to a numerical coefficient of order unity, one has

$$
\Phi_{\mathrm{c}} \simeq \frac{h}{e}\left(\frac{\tau_{\text {ergodic }}}{\tau_{\text {dwell }}}\right)^{1 / 2} \simeq \frac{h}{e}\left(\frac{N \Gamma L^{2} \delta}{h v_{\mathrm{F}} \min (l, L)}\right)^{1 / 2},
$$

where $N$ is the total number of modes in the point contacts, $\Gamma$ the tunnel probability per mode, $L$ the size of the particle, $\delta$ its level spacing, $v_{\mathrm{F}}$ the Fermi velocity, and $l$ the mean free path.

The magnetoconductance has been calculated in the Hamiltonian approach of Sec. II.A.3, by replacing the distribution (82) of the $M \times M$ matrix $\mathcal{H}$ by the distribution (7) of the Pandey-Mehta Hamiltonian $\mathcal{H}=\mathcal{H}_{0}+\mathrm{i} \alpha \mathcal{A}$. In the absence of spin-orbit scattering and $\Gamma=1, N_{1}=N_{2} \gg 1$, the result is (Pluhar et al., 1994, 1995; Frahm, 1995b)

$$
\begin{aligned}
& \left\langle G / G_{0}\right\rangle=\frac{1}{2} N_{1}-\frac{1}{4}\left[1+\left(\Phi / \Phi_{\mathrm{c}}\right)^{2}\right]^{-1}, \\
& \Phi / \Phi_{\mathrm{c}}=2 \alpha \sqrt{M / N_{1}},
\end{aligned}
$$

in agreement with Efetov's (1995) calculation starting from a microscopic Hamiltonian for a disordered metal grain. ${ }^{14}$ The Lorentzian flux dependence in Eq. (111) was first obtained by Baranger, Jalabert, and Stone (1993a, 1993b), using a semiclassical theory (reviewed by Baranger, 1996). [They could not derive the prefactor 1/4 of the Lorentzian, because of an inconsistency in the semiclassical approximation resolved later by Argaman (1995, 1996).] Unlike random-matrix theory, the semiclassical theory can also be applied to the case that the classical motion in the cavity is integrable rather than chaotic. In the integrable case, Baranger, Jalabert, and Stone find a magnetoconductance which is linear, $G(B)-G(0) \propto|B|$, rather than Lorentzian.

The different flux dependence in the chaotic and integrable cases has been observed by Chang et al. (1994). They measured transport through an array of $48 \mathrm{nomi-}$ nally identical quantum dots connected in series and parallel. The quantum dots were fabricated in the twodimensional electron gas of a GaAs/AlGaAs heterostructure. By measuring on an array, the ensemble average of the conductance is obtained directly.

\footnotetext{
${ }^{14}$ Efetov finds for a disordered grain (volume $V$, diffusion coefficient $D$ ) the relation$$
\left(\Phi / \Phi_{c}\right)^{2}=16 \pi^{3}\left(\hbar D / N_{1} \delta\right)(e / h)^{2} V^{-1} \int_{V} d \vec{r}|\vec{A}|^{2}
$$

The gauge of the vector potential $\vec{A}$ is such that the normal component $\hat{n} \cdot \vec{A}$ on the surface of the grain vanishes. This relation agrees with Eqs. (10) and (111b) for a disordered disk or sphere.
}

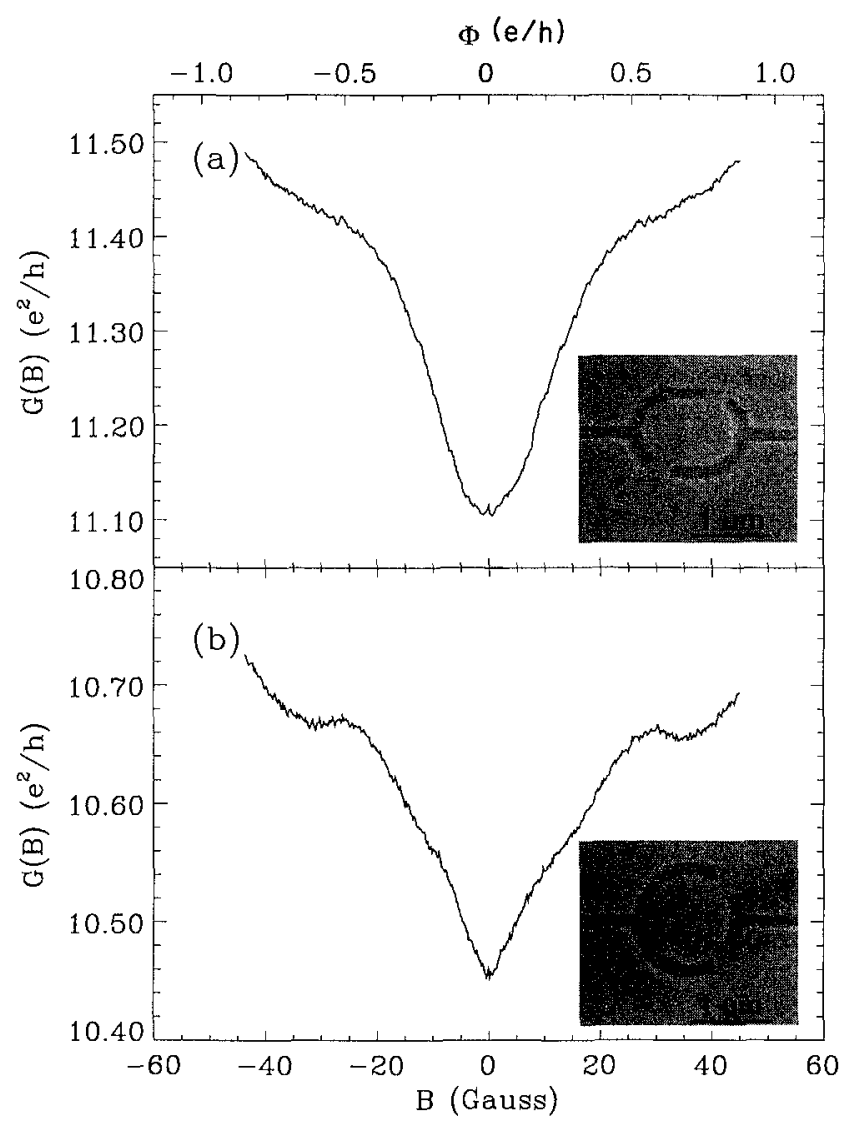

FIG. 8. Magnetoconductance at $50 \mathrm{mK}$, averaged over (a) 48 stadium-shaped cavities and (b) 48 circular-shaped cavities. Insets show the geometry of the cavities, which are fabricated in the two-dimensional electron gas of a GaAs/AlGaAs heterostructure. The weak-localization peak has a Lorentzian shape for the stadium and a triangular shape (linearly decreasing) for the circle, as expected theoretically for, respectively, chaotic and integrable billiards. After Chang et al. (1994).

[Magnetoconductance experiments on a single quantum dot were done by Marcus et al. (1992) and Keller et al. (1994); ensemble averaging by varying the shape of the cavity was done by Chan et al. (1995) and by varying the Fermi energy by Keller et al. (1996).] Two types of arrays were studied, those containing quantum dots in the shape of a stadium, or a circle. (Their area was the same, about $0.8 \mu \mathrm{m}^{2}$, and $N_{1} \approx N_{2} \approx 10$ in both cases.) The classical ballistic motion is chaotic in the stadium and integrable in the circle. The magnetic-field dependence of the conductance in the two cases is shown in Fig. 8. (The conductance of the array has been normalized to that of a single cavity.) The shape of the weaklocalization peak is strikingly different, consistent with the theoretical prediction. Good agreement could be obtained with a numerical simulation that included smallangle scattering by a smooth disorder potential. The measured magnitude $\delta G$ of the peak in the chaotic case is $0.2 G_{0}$, somewhat smaller than the theoretical value of $\frac{1}{4} G_{0}$. (The discrepancy can be accounted for by inelastic scattering, see Sec. II.E.) 


\section{Universal conductance fluctuations}

\section{Conductance}

Weak localization is a quantum correction of order $e^{2} / h$ to the ensemble-averaged conductance. The fluctuations of the conductance from one member of the ensemble to the other are also of order $e^{2} / h$. These fluctuations are known as "universal conductance fluctuations" (see Sec. I.D.2). The magnitude of the conductance fluctuations in a chaotic cavity has been calculated in the Hamiltonian approach by Iida, Weidenmüller, and Zuk (1990a, 1990b), and in the scattering-matrix approach by Baranger and Mello (1994) and Jalabert, Pichard, and Beenakker (1994).

The variance $\operatorname{Var} G=\left\langle G^{2}\right\rangle-\langle G\rangle^{2}$ of the conductance which results from averaging over the circular ensemble of scattering matrices is

$$
\begin{aligned}
\operatorname{Var} G / G_{0}= & 2 \beta^{-1} N_{1} N_{2}\left(N_{1}-1+2 / \beta\right)\left(N_{2}-1+2 / \beta\right) \\
& \times\left(N_{1}+N_{2}-2+2 / \beta\right)^{-1}\left(N_{1}+N_{2}-1+4 / \beta\right)^{-1} \\
& \times\left(N_{1}+N_{2}-1+2 / \beta\right)^{-2} .
\end{aligned}
$$

For $N_{1}, N_{2} \gg 1$ we may expand

$$
\operatorname{Var} G / G_{0}=\frac{2\left(N_{1} N_{2}\right)^{2}}{\beta\left(N_{1}+N_{2}\right)^{4}},
$$

which for two identical contacts simplifies further to

$$
\operatorname{Var} G / G_{0}=\frac{1}{8} \beta^{-1}, N_{1}=N_{2} \gg 1 \text {. }
$$

In Fig. 9 we show experimental data by Chan et al. (1995) for the variance of the conductance of a quantum dot in the two-dimensional electron gas of a GaAs/ AlGaAs heterostructure. An ensemble was constructed by slightly distorting the shape of the quantum dot by means of a gate electrode. (The area of the dot varied by less than $5 \%$ around $2.4 \mu \mathrm{m}^{2}$.) The reduction of the conductance fluctuations by a magnetic field is clearly visible. The two point contacts were adjusted such that $N_{1}=N_{2}=2$. Equation (112) predicts for this case a variance of the conductance equal to $72 / 175 \approx 0.41 \times\left(e^{2} / h\right)^{2}$ for $\beta=1$ and $4 / 15 \approx 0.27 \times\left(e^{2} /\right.$ $h)^{2}$ for $\beta=2$. The experimental values are considerably smaller, mainly as a result of inelastic scattering (see Sec. II.E).

\section{Other transport properties}

To compute the variance of other transport properties than the conductance, one needs the two-point correlation function of the transmission eigenvalues. In the limit $N \rightarrow \infty$ we can use the method of functional derivatives explained in Sec. I.D.1.

The two-point correlation function $K\left(\lambda, \lambda^{\prime}\right)$ $=-\beta^{-1} \delta \rho(\lambda) / \delta V\left(\lambda^{\prime}\right)$ is obtained by variation of the relation between density and potential [Eq. (98)]:

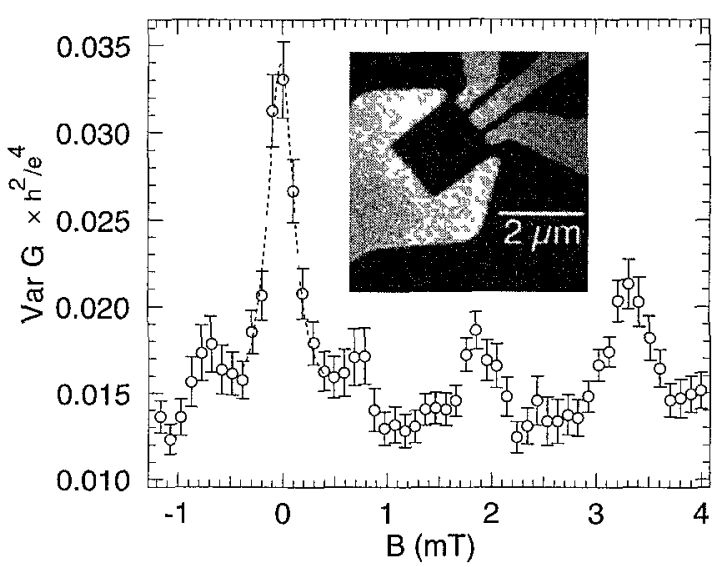

FIG. 9. Variance of the conductance of a quantum dot at $30 \mathrm{mK}$ as a function of magnetic field. The variance is the mean squared of the fluctuation in the conductance as the shape of the quantum dot is distorted. The variance decreases when time-reversal symmetry is broken by a magnetic field. The dashed curve is a fit to a squared Lorentzian. The inset shows an electron micrograph of the device, fabricated in the two-dimensional electron gas of a GaAs/AlGaAs heterostructure. The black rectangle at the center of the inset is the quantum dot, and the gray regions are the gate electrodes on top of the heterostructure. Electrons can enter and exit the quantum dot through point contacts at the top and right corner of the rectangle. The side of the rectangle between these two corners is distorted to generate conductance fluctuations. (The two small openings in the gate along this side are effectively closed in the electron gas.) After Chan et al. (1995).

$$
\begin{aligned}
\delta \rho(\lambda)= & \pi^{-2}\left[\lambda\left(\lambda_{\mathrm{c}}-\lambda\right)\right]^{-1 / 2} \int_{0}^{\lambda_{\mathrm{c}}} d \lambda^{\prime} \delta V\left(\lambda^{\prime}\right) \\
& \times \frac{d}{d \lambda^{\prime}} \frac{\left[\lambda^{\prime}\left(\lambda_{\mathrm{c}}-\lambda^{\prime}\right)\right]^{1 / 2}}{\lambda-\lambda^{\prime}},
\end{aligned}
$$

where we used the parametrization $T_{n}=\left(1+\lambda_{n}\right)^{-1}$. As mentioned in Sec. I.D.1 (footnote), there is no contribution from variation of the boundary $\lambda_{c}=4 N_{1} N_{2}\left(N_{1}-N_{2}\right)^{-2}$ of the spectrum. The variance of the linear statistic $A=\Sigma_{n} a\left(\lambda_{n}\right)$ follows upon integration over the two-point correlation function,

$$
\begin{aligned}
\operatorname{Var} A= & \frac{1}{\beta \pi^{2}} \int_{0}^{\lambda_{\mathrm{c}}} d \lambda \int_{0}^{\lambda_{\mathrm{c}}} \lambda^{\prime} \\
& \times\left(\frac{\lambda^{\prime}\left(\lambda_{\mathrm{c}}-\lambda^{\prime}\right)}{\lambda\left(\lambda_{\mathrm{c}}-\lambda\right)}\right)^{1 / 2} \frac{a(\lambda)}{\lambda-\lambda^{\prime}} \frac{d a\left(\lambda^{\prime}\right)}{d \lambda^{\prime}},
\end{aligned}
$$

where the singular integral is the principal value. For $N_{1}=N_{2}$ one has $\lambda_{\mathrm{c}} \rightarrow \infty$, and Eq. (116) reduces to the result for a logarithmic eigenvalue repulsion in the interval $(0, \infty)$ [Eq. (61)].

\section{Tunnel barriers}

We briefly consider the effect of tunnel barriers on the variance of the conductance. The distribution of the scattering matrix is now the Poisson kernel instead of the circular ensemble. The parametrization of Eq. (105) 
reduces the problem to an integration over the unitary group, which can be done perturbatively for $N \Gamma \gg 1$. The result is (Efetov, 1995; Brouwer and Beenakker, 1996a)

$$
\begin{aligned}
\operatorname{Var} G / G_{0}= & 2 \beta^{-1}\left(g_{1}+g_{1}^{\prime}\right)^{-6}\left(2 g_{1}^{4} g_{1}^{\prime 2}+4 g_{1}^{3} g_{1}^{\prime 3}\right. \\
& -4 g_{1}^{2} g_{2} g_{1}^{\prime 3}+2 g_{1}^{2} g_{1}^{\prime 4}-2 g_{1} g_{2} g_{1}^{\prime 4}+3 g_{2}^{2} g_{1}^{\prime 4} \\
& -2 g_{1} g_{3} g_{1}^{\prime 4}+2 g_{2} g_{1}^{\prime 5}-2 g_{3} g_{1}^{\prime 5}+2 g_{1}^{5} g_{2}^{\prime} \\
& -2 g_{1}^{4} g_{1}^{\prime} g_{2}^{\prime}-4 g_{1}^{3} g_{1}^{\prime 2} g_{2}^{\prime}+6 g_{1}^{2} g_{2} g_{1}^{\prime 2} g_{2}^{\prime} \\
& \left.+3 g_{1}^{4} g_{2}^{\prime 2}-2 g_{1}^{5} g_{3}^{\prime}-2 g_{1}^{4} g_{1}^{\prime} g_{3}^{\prime}\right) .
\end{aligned}
$$

One verifies that Eq. (113) is recovered in the absence of tunnel barriers. For the special case of two identical tunnel barriers $\left(g_{p}=g_{p}^{\prime}\right)$, Eq. (117) reduces to (Iida, Weidenmüller, and Zuk, 1990a, 1990b)

$$
\text { Var } G / G_{0}=\left(8 \beta g_{1}^{2}\right)^{-1}\left(2 g_{1}^{2}-2 g_{1} g_{2}+3 g_{2}^{2}-2 g_{1} g_{3}\right) \text {. }
$$

Another special case is that of high tunnel barriers, $\Gamma_{n} \ll 1$ for all $n$, when Eq. (117) simplifies to (Zirnbauer, 1993)

$$
\operatorname{Var} G / G_{0}=4 \beta^{-1}\left(g_{1}+g_{1}^{\prime}\right)^{-4} g_{1}^{2} g_{1}^{\prime 2} .
$$

Finally, if all transmission eigenvalues $\Gamma_{n} \equiv \Gamma$ are equal, one has $\operatorname{Var} G / G_{0}=(8 \beta)^{-1}\left[1+(1-\Gamma)^{2}\right]$. A high tunnel barrier $(1 / N \ll \Gamma \ll 1)$ doubles the variance.

\section{Magnetoconductance}

A weak magnetic field reduces the variance of the conductance by a factor of two if $N \gg 1$. The dashed line in Fig. 9 is a fit to a squared Lorentzian, which is the theoretical result of Frahm (1995b),

$$
\text { Var } G / G_{0}=\frac{1}{16}+\frac{1}{16}\left[1+\left(\Phi / \Phi_{c}\right)^{2}\right]^{-2}
$$

This result was obtained in the same way as Eq. (111), for a system without spin-orbit scattering and assuming $\Gamma=1, N_{1}=N_{2} \gg 1$. The characteristic flux $\Phi_{\mathrm{c}}$ is related to the parameter $\alpha$ in the Pandey-Mehta Hamiltonian by Eq. (111b), which in turn is related to microscopic parameters by Eqs. (10) and (11). Up to a numerical coefficient of order unity, $\Phi_{c}$ is given by Eq. (110).

Once $\Phi$ is much greater than $\Phi_{c}$, time-reversal symmetry is effectively broken and the variance of the conductance becomes independent of the magnetic field. The conductance of a specific sample fluctuates in a random but reproducible way as a function of magnetic field (see Fig. 4). These magnetoconductance fluctuations (or "magnetofingerprints") are characterized by the correlator

$$
C(\Delta \Phi)=\langle G(\Phi) G(\Phi+\Delta \Phi)\rangle-\langle G(\Phi)\rangle\langle G(\Phi+\Delta \Phi)\rangle,
$$

where $\langle\cdots\rangle$ represents either an ensemble average or an average over $\Phi\left(\gg \Phi_{c}\right)$. This correlator is given by (Efetov, 1995; Frahm, 1995b)

$$
C(\Delta \Phi)=\frac{1}{16} G_{0}^{2}\left[1+\left(\Delta \Phi / 2 \Phi_{\mathrm{c}}\right)^{2}\right]^{-2} \text {. }
$$

The Lorentzian-squared decay of $C(\Delta \Phi)$ was first derived from semiclassical theory by Jalabert, Baranger, and Stone (1990) (reviewed by Baranger, 1996). Experiments by Marcus et al. (1992) (reviewed by Westervelt, 1996) on the magnetoconductance fluctuations of a (chaotic) stadium-shaped quantum dot are in agreement with Eq. (122) and also show the more rapid decay of the correlator for a (nonchaotic) circular geometry predicted by the semiclassical theory.

Efetov (1995) has generalized the zero-temperature result (122) for the correlator to nonzero temperatures. Thermal smearing of the Fermi-Dirac distribution function reduces the magnitude of the magnetoconductance fluctuations, once the thermal energy $k_{\mathrm{B}} T$ becomes greater than the Thouless energy $E_{\mathrm{c} \text {,open }} \simeq N \Gamma \delta$ of the open system. (This is in contrast to the weak-localization effect, which is not influenced by thermal smearing.) In the high-temperature limit $k_{\mathrm{B}} T \gg E_{\mathrm{c}, \text { open }}$ (and for the case $\Gamma=1, \quad N_{1}=N_{2} \gg 1$ ), the correlator becomes a Lorentzian,

$$
C(\Delta \Phi)=\frac{G_{0}^{2}}{96} \frac{N_{1} \delta}{k_{\mathrm{B}} T}\left[1+\left(\Delta \Phi / 2 \Phi_{\mathrm{c}}\right)^{2}\right]^{-1},
$$

instead of a squared Lorentzian.

The results in this subsection all follow from the correlator

$$
\begin{aligned}
C\left(\Phi_{1}, \Phi_{2}, E_{1}, E_{2}\right)= & \left\langle G\left(\Phi_{1}, E_{1}\right) G\left(\Phi_{2}, E_{2}\right)\right\rangle \\
& -\left\langle G\left(\Phi_{1}, E_{1}\right)\right\rangle\left\langle G\left(\Phi_{2}, E_{2}\right)\right\rangle
\end{aligned}
$$

of the (zero-temperature) conductance at two different magnetic fluxes $\Phi_{1}, \Phi_{2}$ and two different values $E_{1}, E_{2}$ of the Fermi energy. This correlator has been derived from the Pandey-Mehta Hamiltonian (Frahm, 1995b) and from a microscopic Hamiltonian (Efetov, 1995). It also follows from semiclassical periodic-orbit theory (Blümel and Smilansky, 1988; Jalabert, Baranger, and Stone, 1990). The result (for $\Gamma=1, N_{1}=N_{2} \gg 1$ )

$$
\begin{aligned}
& C=\frac{1}{16} G_{0}^{2}\left[f\left(\Phi_{\text {tot }}, \Delta E\right)+f(\Delta \Phi, \Delta E)\right], \\
& f(\Phi, E)=\frac{1}{\left[1+\left(\Phi / 2 \Phi_{\mathrm{c}}\right)^{2}\right]^{2}+\left(\pi E / N_{1} \delta\right)^{2}},
\end{aligned}
$$

is a Lorentzian in energy differences $\Delta E=E_{1}-E_{2}$ and a squared Lorentzian in magnetic-flux differences $\Delta \Phi=\Phi_{1}-\Phi_{2}$. The flux dependence also contains a term that depends on the total flux $\Phi_{\text {tot }}=\Phi_{1}+\Phi_{2}$, to ensure that $C$ is an even function of the individual fluxes $\Phi_{1}, \Phi_{2}$.

The correlator (122) in the absence of time-reversal symmetry can also be obtained from the Brownianmotion model for the Hamiltonian of Sec. I.B.4. An altogether different issue is the question of whether a Brownian-motion model for the scattering matrix can describe the magnetoconductance of a chaotic cavity. This issue has been addressed by several authors (Macêdo, 1994b, 1996; Frahm and Pichard, 1995a, 1995b; Rau, 1995). The answer appears to be negative. Rau and Frahm and Pichard find that the effect of a magnetic field on the scattering matrix is a Brownian-motion process only for small flux increments $\Delta \Phi \ll \Phi_{c}$. Macêdo 


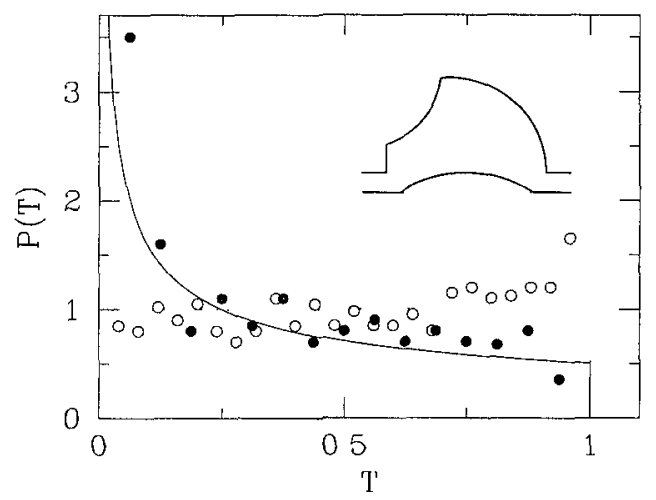

FIG. 10. Distribution of the transmission probability $T$ through a chaotic billiard with two ballistıc single-mode point contacts. Data points are numerical results for the billiard shown in the inset, averaged over a range of Fermi energies and small variations in shape. Filled data points are for $B=0$, open points for $B \neq 0$ (a few flux quanta through the billiard). The solid and dotted curves are the prediction of the circular ensemble for $\beta=1,2$, as given by $\mathrm{Eq}$. (126). After Baranger and Mello (1994).

obtains a Lorentzian-squared decay of the magnetocorrelator from a Brownian-motion model that is not invariant under unitary transformations of the scattering matrix $(S \rightarrow U S)$ and therefore seems unjustifiable.

\section{Conductance distribution}

The conductance of the quantum dot has a Gaussian distribution if the number of modes in the point contacts is large. Deviations from a Gaussian become significant when the fluctuations $\simeq e^{2} / h$ of the conductance become greater than the mean $\approx N \Gamma e^{2} / h$, i.e., when $N \leqslant 1 / \Gamma$.

Consider, as an extreme example, the case $\Gamma=1$, $N_{1}=N_{2}=1$ of two ballistic single-mode point contacts (Baranger and Mello, 1994; Jalabert, Pichard, and Beenakker, 1994). According to Eq. (76), the single transmission eigenvalue $T$ of the quantum dot has probability distribution

$$
P(T)=\frac{1}{2} \beta T^{-1+\beta / 2}, \quad 0<T<1 .
$$

In the presence of a magnetic field $(\beta=2)$, any value of the conductance $G=G_{0} T$ between 0 and $G_{0}\left(=2 e^{2} / h\right)$ is equally probable. In a nonzero field it is more probable to find a small than a large conductance, provided that the scattering preserves spin-rotation symmetry $(\beta=1)$. In the presence of spin-orbit scattering $(\beta=4)$, a large conductance is more probable than a small one. In Fig. 10 we show numerical calculations of transmission through a chaotic billiard by Baranger and Mello (1994, see also Ishio, 1995; Yang, Ishio, and Burgdörfer, 1995), which confirm this remarkable sensitivity of the conductance distribution to a magnetic field.

If the point contacts contain a tunnel barrier, the distribution remains strongly non-Gaussian but becomes less sensitive to a magnetic field. In Fig. 11 we show $P(T)$ for the case of two identical single-mode point

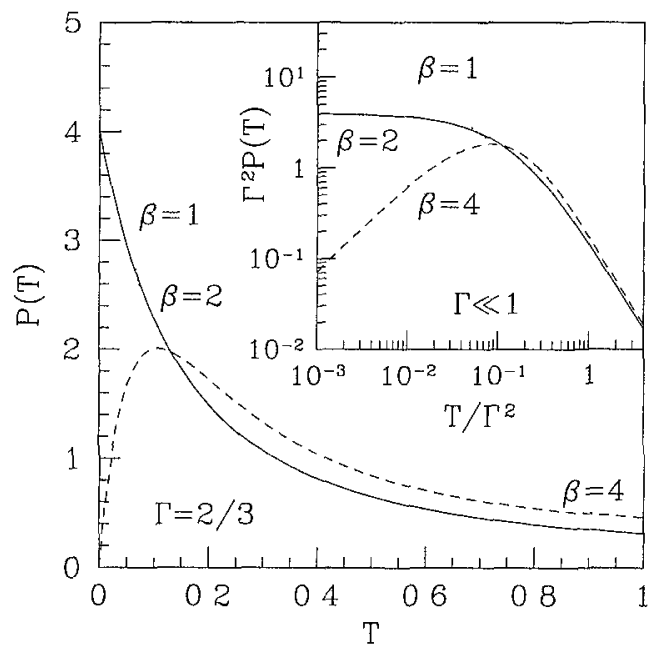

FIG. 11. Distribution of the transmission probability $T$ through a chaotic billiard with two single-mode point contacts containing a tunnel barrier $\left(\Gamma_{1}=\Gamma_{2} \equiv \Gamma\right)$. The curves are computed by integrating over the Poisson kernel, for the three symmetry classes, $\beta=1,2,4$. The main plot is for $\Gamma=2 / 3$, and the inset shows the asymptotic behavior of $P(T)$ for $\Gamma \ll 1$ on a $\log \log$ scale. Notice that the result $P \propto T^{-1+\beta / 2}$ for ballistic point contacts is recovered if $T \ll \Gamma^{2}$. After Brouwer and Beenakker (1994).

contacts with $\Gamma_{1}=\Gamma_{2} \equiv \Gamma=2 / 3$. The inset shows the limit $\Gamma \ll 1$. For $T \gtrsim \Gamma^{2}$ the tunnel barriers dominate the transmission through the entire system, thereby suppressing the $\beta$ dependence of the distribution. For $T \ll \Gamma^{2}$ the presence of tunnel barriers is of less importance, and the $\beta$ dependence remains significant. The curves in Fig. 11 were computed using the Poisson kernel for the distribution of scattering matrices (Brouwer and Beenakker, 1994; Baranger and Mello, 1996a) and agree with results obtained from a tunnel Hamiltonian with disorder (Prigodin, Efetov, and Iida, 1993, 1995). Qualitatively similar results have been obtained by Kamenev and Gefen (1995) for the real part of the frequency-dependent conductance of an isolated metal ring.

Tunnel barriers give a nonzero ensemble-averaged scattering matrix $\bar{S}$ in the Poisson kernel because of direct reflection at a point contact ("direct" meaning without scattering in the cavity). Baranger and Mello (1996a) consider also the case that $\bar{S} \neq 0$ because of direct transmission between the two point contacts. Direct transmission can be achieved by bringing the point contacts close together or by increasing the magnetic field so that electrons can skip along a boundary from one point contact to the other. Good agreement between the Poisson kernel and numerical simulations is found in both cases, if the energy-averaged scattering matrix computed numerically is used as input in the Poisson kernel (see Fig. 12).

Another generalization, considered by Gopar et al. (1996) and Baranger and Mello (1996b), is to quantum dots with a reflection symmetry. (Disordered conductors with a reflection symmetry had been studied earlier by Hastings, Stone, and Baranger, 1994.) The scattering 


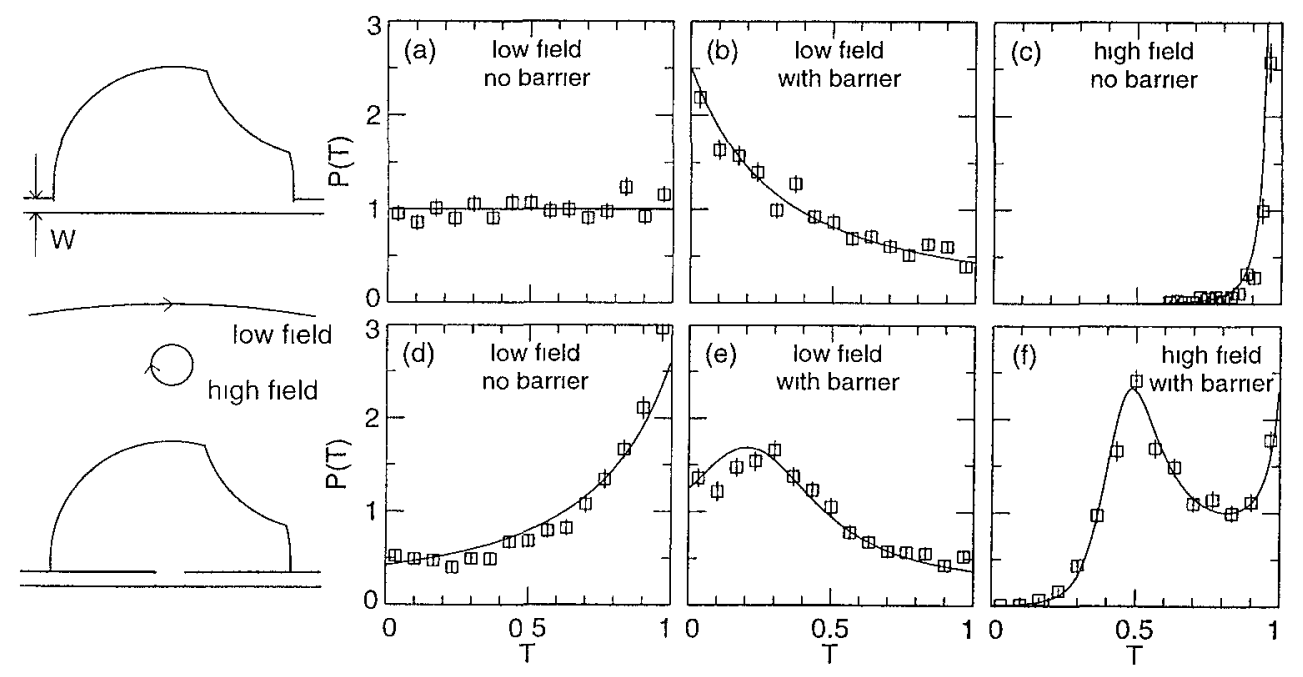

FIG. 12. Distrubution of the transmission probability $T$ for the geometry of Fig. 10 (top row) and for the same geometry with leads extended into the cavity (bottom row). The magnitude of the magnetic field ("low" and "high" corresponding to 2 and 80 flux quanta through the billiard, respectively) and the presence or absence of a tunnel barrier at the entrance to the leads (marked by dotted lines in the sketches of the structures) are noted in each panel. Cyclotron orbits for both fields, drawn to scale, are shown on left. The data points with statistical error bars are numerical results; the curves are the predictions of the Poisson kernel for $\beta=2$, with $\bar{S}$ extracted from the numerical data. After Baranger and Mello (1996a).

matrix for a symmetric geometry decomposes into blocks in a basis of definite parity with respect to the symmetry operator. The blocks have independent distributions in the circular ensemble. Because the conductance couples different blocks, its distribution differs from the result for the circular ensemble [Eq. (126)].

Experiments by Chan et al. (1995) on quantum dots with ballistic point contacts $\left(N_{1}=N_{2}=2\right)$ find a probability distribution for the conductance which is well described by a Gaussian, presumably as a result of inelastic scattering (see Sec. II.E). More recent data by the same group (Marcus, 1996) shows significant deviations from a Gaussian, in particular a distribution which is skewed towards small conductance in zero magnetic field.

\section{E. Phase breaking}

Quantum-interference effects in the conductance require phase coherence of the electron wave function to persist on the time scale $\hbar / E_{\mathrm{c} \text {,open. }}$. If phase coherence is broken after a time $\tau_{\phi}$, then transport becomes classical if $\hbar / \tau_{\phi} \geq E_{\mathrm{c} \text {,open }}$. We discuss two phase-breaking mechanisms-coupling of the quantum dot to the outside through a voltage probe, and inelastic scattering inside the quantum dot. In the latter case, phase breaking occurs uniformly throughout the quantum dot, while in the former case it occurs locally at the voltage probe.

\section{Invasive voltage probe}

The measurement of a voltage at some point in the sample is an invasive act that may destroy the phase coherence throughout the whole sample. The reason is that electrons which enter the voltage lead are reinjected into the system without any phase relationship
(Büttiker, 1986a, 1988a). The phase-breaking effects of a voltage probe on the conductance of a chaotic cavity have been investigated by Baranger and Mello (1995) and Brouwer and Beenakker (1995a, 1997a).

The model consists of a quantum dot that is coupled by two leads to source and drain reservoirs at voltages $V_{1}$ and $V_{2}$. A current $I=I_{1}=-I_{2}$ is passed from source to drain via leads 1 and 2 . A third lead is attached to the quantum dot and connected to a third reservoir at voltage $V_{3}$. This third lead is a voltage probe, which means that $V_{3}$ is adjusted in such a way that no current is drawn $\left(I_{3}=0\right)$. We denote by $N_{l}$ the number of modes in lead $i$, and we assume for simplicity that there are no tunnel barriers in any of the leads.

The scattering matrix $S$ of the system can be written as

$$
S=\left(\begin{array}{lll}
r_{11} & t_{12} & t_{13} \\
t_{21} & r_{22} & t_{23} \\
t_{31} & t_{32} & r_{33}
\end{array}\right),
$$

in terms of reflection and transmission matrices $r_{l}$ and $t_{l j}$ between leads $i$ and $j$. The currents and voltages satisfy (Büttiker, 1986b, 1988b)

$$
\begin{aligned}
& \frac{h}{2 e^{2}} I_{k}=\left(N_{k}-R_{k k}\right) V_{k}-\sum_{l \neq k} T_{k l} V_{l}, \quad k=1,2,3, \\
& R_{k k}=\operatorname{Tr} r_{k k} r_{k k}^{p}, \quad T_{k l}=\operatorname{Tr} t_{k l} t_{k l}^{!} .
\end{aligned}
$$

The two-terminal conductance $G=I /\left(V_{1}-V_{2}\right)$ follows from Eq. (128) with $I_{1}=-I_{2}=I, I_{3}=0$ :

$$
G=\frac{2 e^{2}}{h}\left(T_{12}+\frac{T_{13} T_{32}}{T_{31}+T_{32}}\right) .
$$




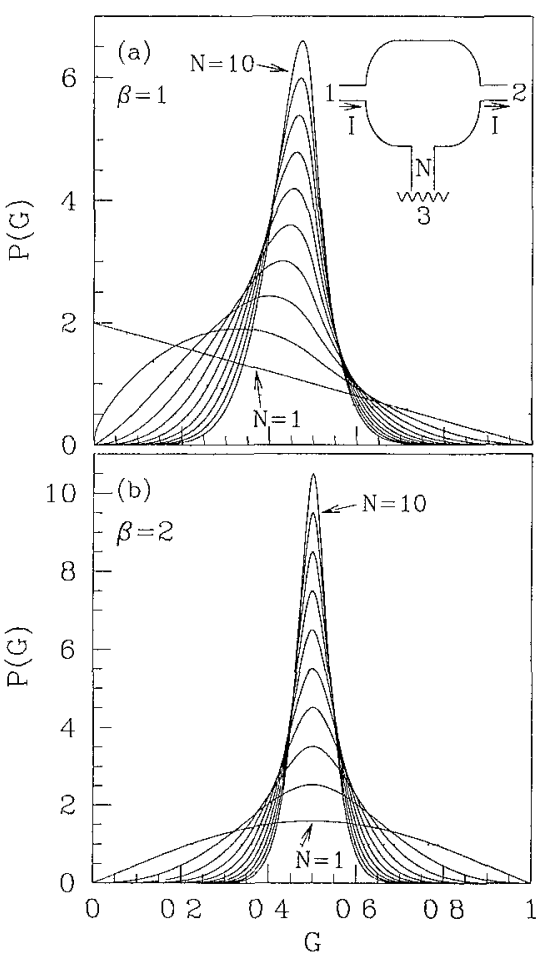

FIG. 13. Effect of an invasive voltage probe on the distribution of the conductance (in units of $2 e^{2} / h$ ). The current-carrying leads 1 and 2 contain a single mode each, while the number of modes $N$ in the voltage lead (labeled 3 in the inset) varies from 1 to 10 with increments of 1 (solid curves). The dotted curve is the distribution (126) in the absence of a voltage lead. The top panel is for $\beta=1$, the bottom panel for $\beta=2$. After Brouwer and Beenakker (1995a).

Analytical results for $P(G)$ can be obtained for $N_{1}=N_{2}=1$ and $N_{3}$ arbitrary (Brouwer and Beenakker, 1995a, 1997a). Because of current conservation

$$
\begin{aligned}
& T_{13}=1-R_{11}-T_{12}=1-\left|S_{11}\right|^{2}-\left|S_{12}\right|^{2}, \\
& T_{31}=1-R_{11}-T_{21}=1-\left|S_{11}\right|^{2}-\left|S_{21}\right|^{2}, \\
& T_{32}=1-R_{22}-T_{12}=1-\left|S_{22}\right|^{2}-\left|S_{12}\right|^{2},
\end{aligned}
$$

so that it sulfices to know the marginal distribution of the matrix elements $S_{k l}$ with $k, l \leqslant 2$. This distribution has been computed by Pereyra and Mello (1983) and Friedman and Mello (1985b). The resulting $P(G)$ is plotted in Fig. 13 for $\beta=1,2$ and $N_{3}$ ranging from 1 to 10. Notice the particularly simple result $P\left(G / G_{0}\right)=2-2 G / G_{0}$ in the case $\beta=1, N_{3}=1$. As $N_{3}$ increases, $P(G)$ becomes more and more sharply peaked around $e^{2} / h$. The limiting distribution as $N_{3} \rightarrow \infty$ is

$$
P\left(G / G_{0}\right)=\frac{1}{2} \beta N_{3}[1+|y|+(1-2 / \beta) y] e^{-|y|},
$$

where we have abbreviated $y=2 \beta N_{3}\left(G / G_{0}-\frac{1}{2}\right)$. Surprisingly enough, the distribution remains non-Gaussian for arbitrarily strong dephasing.

The mean and variance for $N_{3} \gg 1$ can be computed analytically for any $N_{1}=N_{2}$ (Baranger and Mello, 1995),

$$
\left\langle G / G_{0}\right\rangle=\frac{1}{2} N_{1}+\frac{1}{2}(1-2 / \beta) N_{1} / N_{3},
$$

$$
\operatorname{Var} G / G_{0}=\frac{2 N_{1}+2-\beta}{4 \beta N_{1}}\left(N_{1} / N_{3}\right)^{2} .
$$

The variance of $G$ is reduced by a factor of $2+1 / N_{1}$ when time-reversal symmetry is broken in the limit $N_{3} \rightarrow \infty$. The offset of $\left\langle G / G_{0}\right\rangle$ from $\frac{1}{2} N_{1}$ when $\beta=1$ is a remnant of the weak-localization effect.

\section{Inelastic scattering}

The phase-breaking effects of inelastic scattering (scattering rate $1 / \tau_{\phi}$ ) can be modeled by an imaginary voltage lead with $N_{3}=2 \pi \hbar / \tau_{\phi} \delta$ (Marcus $e t$ al., 1993). In this way, Baranger and Mello (1995) have been able to account for the discrepancies between the predicted and measured magnitude of quantum corrections mentioned in Secs. II.B.4, II.C.1, and II.D. An alternative model, which includes a (spatially uniform) imaginary potential in the Hamiltonian, equal to $-\frac{1}{2} i \hbar / \tau_{\phi}$, was used by Efetov (1995) and McCann and Lerner (1996). The two models give very different results for the distribution of the conductance, in particular in the case that the current through the quantum dot flows through singlemode point contacts. While the distribution $P(G)$ becomes a delta peak at the classical conductance for very strong dephasing $\left(\tau_{\phi} \rightarrow 0\right)$ in the voltage-probe model, $P(G)$ peaks at zero conductance in the imaginarypotential model.

The origin of the difference lies with certain shortcomings of each model. On the one hand, the imaginarypotential model does not conserve the number of electrons. On the other hand, the voltage-probe model describes spatially localized instead of spatially uniform dephasing. There exists a limit of the voltage-probe model that applies to dephasing processes occurring uniformly in space (Brouwer and Beenakker, 1997a). This limit is equivalent to a particle-conserving version of the imaginary-potential model. What one needs to do is introduce a tunnel barrier (transparency $\Gamma_{3}$ ) in the voltage probe and take the limit $N_{3} \rightarrow \infty, \Gamma_{3} \rightarrow 0$ at fixed $N_{3} \Gamma_{3}=2 \pi \hbar / \tau_{\phi} \delta$. The resulting conductance distribution narrows around the classical series conductance of the two point contacts when $\tau_{\phi} \rightarrow 0$, in a way which is similar, but not precisely identical, to the voltage-probe model with $\Gamma_{3}=1$.

Neither the voltage-probe model nor the imaginarypotential model provides a microscopic description of electron-electron scattering, which is the main source of inelastic scattering at low temperatures. At present there exists a microscopic theory for dephasing by electron-electron interactions in closed systems (Sivan, Imry, and Aronov, 1994; Altshuler, Gefen, Kamenev, and Levitov, 1997), but not yet in open systems.

\section{F. Coulomb blockade}

So far we have ignored the Coulomb repulsion of electrons in the quantum dot. A measure of the importance of Coulomb repulsion is the charging energy $e^{2} / 2 C$ of a single electron in the quantum dot (capaci- 
tance $C$ ). The charging energy plays no role if the quantum dot is strongly coupled to the reservoirs, but it does if the coupling is weak. The strength of the coupling is determined by whether the broadening $\gamma$ of the energy levels in the quantum dot is large or small compared to their spacing $\delta$. The ratio $\gamma / \delta$ is of the order of the conductance $G$ of the quantum dot in units of $e^{2} / h$, so that Coulomb repulsion is important if $G \leqslant e^{2} / h$. In addition, the charging energy should be large compared to the thermal energy $k_{\mathrm{B}} T$. If both of these conditions are met, the conductance oscillates as a function of the Fermi energy, with periodicity $e^{2} / C$ (Shekhter, 1972; Kulik and Shekhter, 1975). The periodic suppression of the conductance is known as the Coulomb blockade. There exist several reviews devoted entirely to this phenomenon (Averin and Likharev, 1991; Van Houten, Beenakker, and Staring, 1992; Meirav and Foxman, 1995). Here we discuss one aspect of it, for which random-matrix theory is relevant (Jalabert, Stone, and Alhassid, 1992).

If $k_{\mathrm{B}} T \ll e^{2} / C$ the oscillations of the conductance develop into a sequence of well-resolved peaks. If, moreover, $k_{\mathrm{B}} T \ll \delta$, a single energy level $E_{i}$ in the quantum dot contributes to each peak. The amplitude of the peaks fluctuates because of fluctuations in the wave functions of subsequent levels. ${ }^{15}$ If $k_{\mathrm{B}} T \gg \gamma$ the peak amplitude can be calculated using rate equations (Averin, Korotkov, and Likharev, 1991; Beenakker, 1991). [At lower temperatures complications arise because of the Kondo effect ( $\mathrm{Ng}$ and Lee, 1988; Meir, Wingreen, and Lee, 1991).] The result for the height $G_{\max }$ of the $i$ th conductance peak is

$$
G_{\max }=\frac{e^{2}}{h} \frac{\pi}{2 k_{\mathrm{B}} T} \frac{\sum_{n=1}^{N_{1}} \sum_{n^{\prime}=N_{1}+1}^{N_{1}+N_{2}} \gamma_{n}^{(i)} \gamma_{n^{\prime}}^{(i)}}{\sum_{n=1}^{N_{1}+N_{2}} \gamma_{n}^{(i)}},
$$

where $\gamma_{n}^{(i)} / \hbar$ is the tunnel rate from level $i$ in the quantum dot to mode $n$ in one of the two leads. In terms of the Hamiltonian (80), the tunnel rate $\gamma_{n}^{(i)}$ is determined by the eigenvalue $w_{n}$ of the coupling-matrix product $W W^{\dagger}$ and by the matrix $U$ that diagonalizes the Hamiltonian $\mathcal{H}=U$ diag $\left(E_{1}, E_{2}, \ldots, E_{M}\right) U^{\dagger}$ of the isolated quantum dot. In a basis in which $W W^{\dagger}$ is diagonal, the relation reads

$$
\gamma_{n}^{(i)}=2 \pi w_{n}\left|U_{n i}\right|^{2}=\frac{\Gamma_{n} M \delta}{2 \pi}\left|U_{n i}\right|^{2} .
$$

Equation (135) follows from the scattering matrix (81), under the assumption that $w_{n} \ll M \delta$, which in view of Eq. (84) implies $\Gamma_{n} \ll 1$. Since $\left|U_{n i}\right|^{2} \simeq 1 / M$, this also implies $\gamma_{n}^{(i)} \ll \delta$. Substitution of Eq. (135) into Eq. (134) gives

\footnotetext{
${ }^{15}$ The amplitude of the minima of the conductance oscillations also fluctuates. These fluctuations involve virtual transitions to excited states in the quantum dot (Averin and Nazarov, 1990) and hence depend on the statistics of the superposition of a large number of wave functions (Aleiner and Glazman, 1996).
}

$$
G_{\max }=\frac{e^{2}}{h} \frac{M \delta}{4 k_{\mathrm{B}} T} \frac{\sum_{n=1}^{N_{1}} \sum_{n^{\prime}=N_{1}+1}^{N_{1}+N_{2}} \Gamma_{n} \Gamma_{n^{\prime}}\left|U_{n i}\right|^{2}\left|U_{n^{\prime} i}\right|^{2}}{\sum_{n=1}^{N_{1}+N_{2}} \Gamma_{n}\left|U_{n i}\right|^{2}} .
$$

Peak-to-peak fluctuations in $G_{\max }$ are due to level-tolevel fluctuations in the eigenfunctions of the quantum dot at the two tunnel barriers, represented by the vector $\vec{U} \equiv\left(U_{1 i}, U_{2 i}, \ldots, U_{N i}\right)$ of length $N=N_{1}+N_{2}$. The probability distribution $P\left(G_{\max }\right)$ of the peak heights follows from the distribution $P(\vec{U})$ in the limit $M \rightarrow \infty$ at fixed $N$. The distribution $P(\vec{U})$, in turn, follows from the distribution of the matrix $U$. In zero magnetic field (without spin-orbit scattering, $\beta=1$ ), the real matrix $U$ is uniformly distributed in the orthogonal group. In a magnetic field $(\beta=2)$, the complex matrix $U$ is uniformly distributed in the unitary group. The resulting distribution of $\vec{U}$ factorizes for $M \gg N$ into independent Gaussian distributions with zero mean and variance $1 / \beta M$ (see Appendix B):

$$
P(\vec{U})=(\beta M / 2 \pi)^{\beta N / 2} \exp \left(-\frac{1}{2} \beta M \sum_{n=1}^{N}\left|U_{n i}\right|^{2}\right) .
$$

The distribution $P\left(G_{\max }\right)$, which follows from Eqs. (136) and (137), takes on a simple form if $N_{1}=N_{2}=1$, $\Gamma_{1}=\Gamma_{2} \equiv \Gamma$ (Jalabert, Stone, and Alhassid, 1992; Prigodin, Efetov, and Iida, 1993),

$$
\begin{aligned}
& P(g)=\left\{\begin{array}{l}
(\pi g)^{-1 / 2} e^{-g}, \quad \beta=1, \\
g\left[K_{0}(g)+K_{1}(g)\right] e^{-g}, \quad \beta=2,
\end{array}\right. \\
& g \equiv G_{\max } \frac{h}{e^{2}} \frac{8 k_{\mathrm{B}} T}{\Gamma \delta} .
\end{aligned}
$$

Here $K_{0}$ and $K_{1}$ are Bessel functions. [The case $N_{1}, N_{2}>1$ has been considered by Mucciolo, Prigodin, and Altshuler (1995) and by Alhassid and Lewenkopf (1995).] The distribution (138) has been confirmed experimentally by Chang et al. (1996) and by Folk et al. (1996). Measurements by Chang et al. are shown in Fig. 14. Good agreement is found with Eq. (138), using a single adjustable parameter.

Folk et al. also measured the correlator

$$
C_{\max }(\Delta B)=\left\langle G_{\max }(B) G_{\max }(B+\Delta B)\right\rangle-\left\langle G_{\max }(B)\right\rangle^{2}
$$

of the height of a given peak at different magnetic fields. To obtain this correlator theoretically, one can use the Brownian-motion model described in Sec. I.B.4. If the field $B$ is large enough to break time-reversal symmetry, the matrix elements $\mathcal{H}_{n m}$ of the Hamiltonian of the closed system execute a Brownian motion in the Gaussian unitary ensemble, in the fictitious time $\tau \propto(\Delta B)^{2}$. The problem is to extract the evolution of the matrix of eigenfunctions $U$ from the Brownian motion of $\mathcal{H}$. This problem has been studied by Alhassid and Attias (1996) and by Bruus, Lewenkopf, and Mucciolo (1996). An analytical solution exists only for $\Delta B$ small compared to 


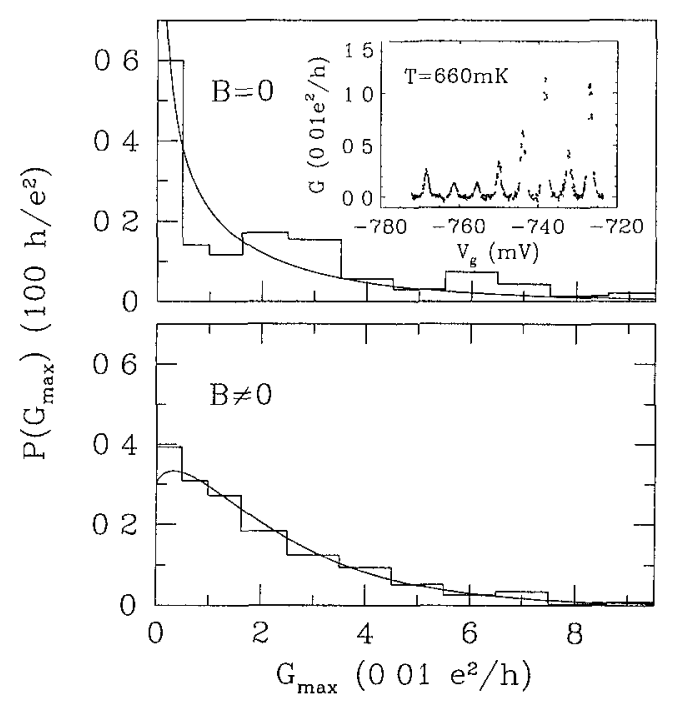

FIG. 14. Histograms measure the probability distributions of the peak heights $G_{\max }$ at $75 \mathrm{mK}$, with and without a timereversal-symmetry-breaking magnetic field. The curves are a one-parameter fit to Eq. (138) for $\beta=1,2$ (same parameter value $\Gamma \delta=0.27 k_{\mathrm{B}} T$ in both curves). Inset: Conductance at $660 \mathrm{mK}$ of a quantum dot in the two-dimensional electron gas of a GaAs/AlGaAs heterostructure as a function of the voltage on a gate electrode controlling the number of electrons in the dot in equilibrium (about 100, in an area of $0.25 \mu \mathrm{m} \times 0.25 \mu \mathrm{m}$; the system is chaotic because of weak disorder). A conductance peak occurs each time this number increases by one because then it costs no charging energy to tunnel into the dot. After Chang et al. (1996).

the correlation field $B_{\mathrm{c}}$. Numerical calculations suggest $C_{\max }(\Delta B) \propto\left[1+\left(\Delta B / B_{c}\right)^{2}\right]^{-2}$, which is roughly in agreement with experiment.

\section{G. Frequency dependence}

Throughout this review we focus on zero-frequency (DC) transport properties. The generalization to nonzero frequencies $\omega$ in the case of a quantum dot is briefly discussed in this subsection, following Gopar, Mello, and Büttiker (1996), and Brouwer and Büttiker (1997). Variations in the currents $I_{i}(\omega)$ and voltages $V_{j}(\omega)$ in the two leads $(i, j=1,2)$ are related by the conductance coefficients $G_{l j}(\omega)=\partial I_{l} / \partial V_{J}$. At zero frequency, current conservation implies that $G_{11}=G_{22}=-G_{12}=-G_{21}$ equals the DC conductance $G$. At nonzero frequency all four conductance coefficients are different in general (Büttiker, 1993; Büttiker, Prêtre, and Thomas, 1993; Büttiker and Christen, 1996). If we ignore the screening of charges accumulated temporarily in the system, the conductance coefficients are related to the scattering matrix by

$$
\begin{aligned}
G_{l j}^{\infty}= & \frac{2 e^{2}}{h} \int_{-\infty}^{\infty} \frac{d \varepsilon}{\hbar \omega}\left[f\left(\varepsilon-\frac{1}{2} \hbar \omega\right)-f\left(\varepsilon+\frac{1}{2} \hbar \omega\right)\right] \\
& \times \operatorname{Tr}\left[\delta_{l j}-S_{l j}^{\dagger}\left(\varepsilon-\frac{1}{2} \hbar \omega\right) S_{l J}\left(\varepsilon+\frac{1}{2} \hbar \omega\right)\right],
\end{aligned}
$$

where $f(\varepsilon)=\left[1+\exp \left(\varepsilon / k_{\mathrm{B}} T\right)\right]^{-1}$ is the Fermi function. The $N_{l} \times N_{j}$ matrix $S_{l j}(\varepsilon)$ contains the scattering amplitudes from lead $j$ into lead $i$ at energy $\varepsilon$, measured relative to the Fermi energy. Neglecting screening amounts to putting the capacitance $C$ of the system equal to infinity, hence the superscript $\infty$ on $G_{i j}$.

Prigodin et al. $(1994,1995)$ have computed the average $\left\langle G_{12}^{\infty}\right\rangle$ for the single-mode case $N_{1}=N_{2}=1$. The case $N_{1}, N_{2} \gg 1$ was considered by Brouwer and Büttiker (1997), who found

$$
\begin{aligned}
\frac{h}{2 e^{2}}\left\langle G_{\imath \jmath}^{\infty}\right\rangle= & \delta_{\iota \jmath} N_{\iota}-\frac{N_{l} N_{\jmath}}{N\left(1-i \omega \tau_{\mathrm{dwell}}\right)}-\frac{(1-2 / \beta) N_{l}}{N\left(1-i \omega \tau_{\mathrm{dwell}}\right)} \\
& \times\left(\frac{N_{J}\left(1-2 i \omega \tau_{\mathrm{dwell}}\right)}{N\left(1-i \omega \tau_{\mathrm{dwell}}\right)^{2}}-\delta_{\imath \jmath}\right),
\end{aligned}
$$

where $N=N_{1}+N_{2}$ and $\tau_{\text {dwell }}=2 \pi \hbar / N \delta$ is the mean dwell time of an electron in the quantum dot. One can check that Eq. (94) is recovered in the limit $\omega \rightarrow 0$.

Screening is irrelevant for the DC conductance but has an essential effect on the frequency dependence. If the potential inside the quantum dot can be assumed to be spatially uniform, the conductance coefficients take the form (Büttiker, Prêtre, and Thomas, 1993)

$$
G_{l l}=G_{l j}^{\infty}+\frac{\sum_{k, l=1}^{2} G_{l k}^{\infty} G_{l j}^{\infty}}{i \omega C-\sum_{k, l=1}^{2} G_{k l}^{\infty}},
$$

so that Eq. (140) is recovered in the limit $C \rightarrow \infty$. Since fluctuations in $G_{l j}^{\infty}$ are of relative order $N^{-2}$, we may directly substitute Eq. (141) into Eq. (142), to obtain the average

$$
\begin{aligned}
\frac{h}{2 e^{2}}\left\langle G_{\imath \jmath}\right\rangle= & \delta_{l j} N_{\iota}-\frac{N_{l} N_{\jmath}}{N\left(1-i \omega \tau_{C}\right)}-\frac{(1-2 / \beta) N_{\imath}}{N\left(1-i \omega \tau_{\mathrm{dwell}}\right)} \\
& \times\left(\frac{N_{\jmath}\left(1-2 i \omega \tau_{C}\right)}{N\left(1-i \omega \tau_{C}\right)^{2}}-\delta_{\imath \jmath}\right)
\end{aligned}
$$

with $1 / \tau_{C}=1 / \tau_{\text {dwell }}+2 e^{2} N / h C$. The $\mathcal{O}(N)$ term in Eq. (143) is the classical Drude conductance, with $\tau_{C}$ playing the role of the $R C$ time of the circuit. The $\beta$-dependent $\mathcal{O}(1)$ term is the frequency-dependent weak-localization correction. For $C \rightarrow 0$, the $R C$ time $\tau_{C}$ vanishes. In this limit all four conductance coefficients are the same, $G_{11}=G_{22}=-G_{12}=-G_{21} \equiv G$, with average

$$
\frac{h}{2 e^{2}}\langle G\rangle=\frac{N_{1} N_{2}}{N}+\frac{(1-2 / \beta) N_{1} N_{2}}{N^{2}\left(1-i \omega \tau_{\text {dwell }}\right)} .
$$

The frequency dependence of the conductance is now due entirely to the weak-localization effect.

\section{DISORDERED WIRES}

\section{A. Dorokhov-Mello-Pereyra-Kumar equation}

\section{Scaling approach to localization}

The scaling approach to localization (Abrahams et al., 1979) studies the limiting behavior of the conductance as 
one or more of the dimensions of the system tends toward infinity. Classically, Ohm's law tells us that the conductance $G \propto L^{d-2}$ in an $L \times L \times L$ cube $(d=3)$, an $L \times L$ square $(d=2)$, or a chain of length $L(d=1)$. The fundamental result of Abrahams et al. (1979) is that this classical scaling is only valid in three dimensions and for sufficiently weak disorder. For $d=3$ and strong disorder, or for $d=1$ and any disorder strength, the conductance $G \propto \exp (-L / \xi)$ decays exponentially for large $L$. Two dimensions is the marginal case $(G \rightarrow 0$ as $L \rightarrow \infty$ for $d=2$ in the absence of spin-orbit scattering, but the decay is not necessarily exponential). The localization length $\xi$ depends on the mean free path $l$. For a chain, $\xi \leqq l$. For a cube, $\xi$ diverges with some power of $1 /\left(l_{\mathrm{c}}-l\right)$ as $l$ increases towards a critical value $l_{\mathrm{c}}$, which is of the order of the Fermi wavelength $\lambda_{\mathrm{F}}$. At $l=l_{\mathrm{c}}$ a transition occurs from a metal $\left(l>l_{\mathrm{c}}\right)$ to an insulator $\left(l<l_{\mathrm{c}}\right)$. This disorder-induced metal-insulator transition is known as the Anderson transition (for reviews, see Lee and Ramakrishnan, 1985; Brezini and Zekri, 1992; Vollhardt and Wölfle, 1992; Kramer and MacKinnon, 1993). No metal-insulator transition occurs for $d=1$ (or for $d=2$ in the absence of spin-orbit scattering). In one dimension the system scales towards an insulator even in the case $l \gg \lambda_{F}$ of weak disorder.

The 1979 paper of Abrahams et al. was based on a qualitative relationship between the conductance of an open system and the response to a change in boundary conditions of eigenstates of the corresponding closed system (Edwards and Thouless, 1972; Thouless, 1977). In 1980, Anderson et al. proposed a "New method for a scaling theory of localization," based on the more precise relationship between the conductance and the scattering states of the open system (Landauer, 1957, 1970). They considered a one-dimensional (1D) chain with weak scattering $\left(l \gg \lambda_{F}\right)$ and computed how the transmission probability $T$ (and hence the conductance $\left.G=T \times 2 e^{2} / h\right)$ scales with the chain length $L$. For $L>l$ an exponential decay was obtained, demonstrating localization. In the following decade the scaling theory of 1D localization was developed in great detail (Abrikosov, 1981; Mel'nikov, 1981; Kirkman and Pendry, 1984; Kumar, 1985; Mello, 1986) and the complete distribution $P(T, L)$ of the transmission probability was found. This solved the problem of $1 \mathrm{D}$ localization due to weak disorder (for the opposite regime of strong disorder, see the reviews by Erdös and Herndon, 1982 and Pendry, 1994).

A real metal wire is not one dimensional. Typically, the width $W$ is much greater than $\lambda_{F}$, so that the number $N$ of transverse modes at the Fermi level is much greater than one. Instead of a single transmission probability $T$, one now has the $N$ eigenvalues $T_{n}$ of the transmission matrix product $t t^{\dagger}$. To obtain the distribution of the conductance $G=\left(2 e^{2} / h\right) \Sigma_{n} T_{n}$ one now needs the joint probability distribution $P\left(T_{1}, T_{2}, \ldots, T_{N}, L\right)$. This distribution differs essentially from the distribution in the $1 \mathrm{D}$ chain, because of correlations induced by the repulsion of nearby eigenvalues. As a consequence of the eigenvalue repulsion, the localization length $\xi \simeq N l$ is increased by a factor of $N$ in comparison to the 1D case (Thouless, 1977). One can therefore distinguish a metallic and an insulating regime. On length scales $l \ll L \ll N l$ the conductance decreases linearly rather than exponentially with $L$. This is the (diffusive) metallic regime, where mesoscopic effects such as weak localization and universal conductance fluctuations occur. The insulating regime of exponentially small conductance is entered for wire lengths $L \geqq N l$.

A scaling theory of localization in multimode wires was pioneered by Dorokhov (1982) and independently by Mello, Pereyra, and Kumar (1988). The DMPK equation,

$$
\begin{aligned}
& l \frac{\partial P}{\partial L}=\frac{2}{\beta N+2-\beta} \sum_{n=1}^{N} \frac{\partial}{\partial \lambda_{n}} \lambda_{n}\left(1+\lambda_{n}\right) J \frac{\partial}{\partial \lambda_{n}} \frac{P}{J}, \\
& J=\prod_{l=1}^{N} \prod_{J=l+1}^{N}\left|\lambda_{l}-\lambda_{l}\right|^{\beta},
\end{aligned}
$$

describes the evolution with increasing wire length of the distribution function $P\left(\lambda_{1}, \lambda_{2}, \ldots, \lambda_{N}, L\right)$. [We recall the definition of the variables $\lambda_{n}=\left(1-T_{n}\right) / T_{n}$.] Equation (145) is known as a Fokker-Planck equation (or generalized diffusion equation) in the theory of Brownian motion (Van Kampen, 1981). One can say that the DMPK equation is a description of scaling in a multimode wire as the Brownian motion of transmission eigenvalues.

For a $1 \mathrm{D}$ chain $(N=1)$ the Jacobian $J \equiv 1$, and Eq. (145) simplifies to

$$
l \frac{\partial}{\partial L} P(\lambda, L)=\frac{\partial}{\partial \lambda} \lambda(1+\lambda) \frac{\partial}{\partial \lambda} P(\lambda, L)
$$

independent of the symmetry index $\beta$. The diffusion equation (146) was derived and solved as early as 1959 by Gertsenshtein and Vasil'ev in an article entitled "Waveguides with random inhomogeneities and Brownian motion in the Lobachevsky plane." This remarkable paper on the exponential decay of radio waves due to weak disorder contains many of the results that were rediscovered in the eighties for the problem of $1 \mathrm{D}$ localization of electrons (see the references listed above). The paper was noticed in the literature on classical wave propagation (Gazaryan, 1969; Papanicolaou, 1971) but apparently not among solid-state physicists.

\section{Brownian motion of transmission eigenvalues}

Equation (145) was derived by Dorokhov (1982, for $\beta=2$ ), and by Mello, Pereyra, and Kumar (1988, for $\beta=1$, with generalizations to $\beta=2,4$ by Mello and Stone, 1991, and Macêdo and Chalker, 1992), by computing the incremental change of the transmission eigenvalues upon attachment of a thin slice to the wire. It is assumed that the conductor is weakly disordered $\left(l \gg \lambda_{\mathrm{F}}\right)$, so that the scattering in the thin slice can be treated perturbatively. A key simplification is the isotropy assumption that the flux incident in one scattering channel is, on 


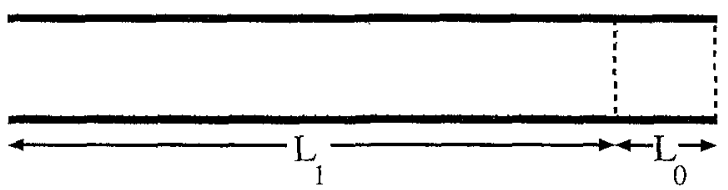

FIG. 15. Disordered wire of length $L_{1}$ to which a segment of length $L_{0}$ is attached. This scaling operation leads to a Brownian motion of the transmission eigenvalues.

average, equally distributed among all outgoing channels. This assumption restricts the applicability of the DMPK equation to a wire geometry $(L \gg W)$, since it ignores the finite time scale for transverse diffusion. The derivation of the DMPK equation given in this subsection emphasizes the fact that it holds on length scales $\gg l$ regardless of the microscopic scattering properties of the conductor. It is similar in spirit to the derivation given by Mello and Shapiro (1988). An altogether different derivation has been given by Tartakovski (1995).

We consider a wire of length $L_{1}$, to which we attach a segment of length $L_{0}$ (see Fig. 15). The combined system, of length $L_{2}=L_{1}+L_{0}$, has transmission matrix

$$
t_{2}=t_{1}\left(1-r_{0} r_{1}\right)^{-1} t_{0}
$$

where $t_{l}$ and $r_{l}$ are $N \times N$ transmission and reflection matrices of the segment of length $L_{i}$. Equation (147) ignores the propagation of evanescent modes, which is justified if $L_{0} \gg \lambda_{\mathrm{F}}$. We denote by $T_{n}(n=1,2, \ldots, N)$ the eigenvalues of the transmission-matrix product $t_{1} t_{1}^{\dagger}$, and by $T_{n}+\delta T_{n}$ the eigenvalues of $t_{2} t_{2}^{\dagger}$. If $L_{0} \ll l$, the change $\delta T_{n}$ of the transmission eigenvalues can be computed by perturbation theory. In view of the earlier requirement $L_{0} \gg \lambda_{\mathrm{F}}$, this implies a restriction to weak scattering, $l \gg \lambda_{\mathrm{F}}$.

To second order in perturbation theory one has

$$
\delta T_{n}=w_{n n}+\sum_{m(\neq n)} \frac{\left|w_{n m}\right|^{2}}{T_{n}-T_{m}}+\mathcal{O}\left(L_{0} / l\right)^{3 / 2}
$$

The matrix element $w_{n m}$ [of order $\left.\left(L_{0} / l\right)^{1 / 2}\right]$ is an element of the Hermitian matrix $w=t_{2} t_{2}^{\dagger}-t_{1} t_{1}^{\dagger}$ in the basis where $t_{1} t_{1}^{b}$ is diagonal. To determine $w$ in this basis we use a polar decomposition of the transmission and reflection matrices [see Eq. (24)],

$$
\begin{array}{ll}
t_{0}=U_{0} \sqrt{\mathcal{T}_{0}} V_{0}^{\prime}, & r_{0}=U_{0} \sqrt{1-\mathcal{T}_{0}} U_{0}^{\prime}, \\
t_{1}=U_{1} \sqrt{\mathcal{T}_{1}} V_{1}^{\prime}, & r_{1}=V_{1} \sqrt{1-\overline{\mathcal{T}_{1}}} V_{1}^{\prime} .
\end{array}
$$

The $U$ 's and $V$ 's are $N \times N$ unitary matrices, and the diagonal matrices $\mathcal{T}_{l}$ contain the $N$ transmission eigenvalues of segment $i$. Combining Eqs. (147) and (149), and noting that $1-\mathcal{T}_{0}=\mathcal{O}\left(L_{0} / l\right)$, one obtains the expansion

$$
\begin{aligned}
w= & \sqrt{\mathcal{T}_{1}} V \sqrt{1-\mathcal{T}_{0}} U \sqrt{1-\mathcal{T}_{1}} \sqrt{\mathcal{T}_{1}}+\text { H.c. } \\
& +\sqrt{\mathcal{T}_{1}}\left(V \sqrt{1-\mathcal{T}_{0}} U \sqrt{1-\mathcal{T}_{1}}\right)^{2} \sqrt{\mathcal{T}_{1}}+\text { H.c. } \\
& +\sqrt{\mathcal{T}_{1}} V \sqrt{1-\mathcal{T}_{0}} U\left(1-\mathcal{T}_{1}\right) U^{\dagger} \sqrt{1-\mathcal{T}_{0}} V^{\dagger} \sqrt{\mathcal{T}_{1}} \\
& -\sqrt{\mathcal{T}_{1}} V\left(1-\mathcal{T}_{0}\right) V^{\dagger} \sqrt{\mathcal{T}_{1}}+\mathcal{O}\left(L_{0} / l\right)^{3 / 2}
\end{aligned}
$$

with the definitions $U=U_{0}^{\prime} V_{1}, V=V_{1}^{\prime} U_{0}$. The abbreviation H.c. stands for Hermitian conjugate.

We now assume that the segment of length $L_{0}$ is taken from an ensemble with an isotropically distributed scattering matrix. This means that the unitary matrices in the polar decomposition (149a) are uniformly distributed in the unitary group. The matrix of transmission eigenvalues $\mathcal{T}_{0}$ may have an arbitrary distribution. The mean free path $l$ is defined in terms of its first moment,

$$
\left\langle\operatorname{Tr} \mathcal{T}_{0}\right\rangle=N\left(1-L_{0} / l\right) .
$$

We will see in Sec. III.B.1 that this definition differs by a numerical coefficient (dependent on the dimensionality of the Fermi surface) from that of the transport mean free path $l_{\mathrm{tr}}$ of kinetic theory:

$$
\frac{l}{l_{\mathrm{tr}}}= \begin{cases}2 & (1 \mathrm{D} \text { chain }) \\ \pi / 2 & (\text { Fermi circle }) \\ 4 / 3 & (\text { Fermi sphere })\end{cases}
$$

The ensemble average $\langle\cdots\rangle$ can be performed in two steps, first averaging over the unitary matrices and then over the transmission eigenvalues. In the absence of time-reversal symmetry $(\beta=2)$, the matrices $U$ and $V$ are independent, hence $\langle f(U, V)\rangle$ $=\int d \mu(U) \int d \mu(V) f(U, V)$. In the presence of timereversal symmetry $(\beta=1$; the case $\beta=4$ requires separate treatment $\left.{ }^{16}\right)$, the matrices $U$ and $V$ are each others transpose, hence $\langle f(U, V)\rangle=\int d \mu(U) f\left(U, U^{\mathrm{T}}\right)$. The integrals over the unitary group [with invariant measure $d \mu(U)]$ can be performed with the help of the formulas in Appendix B.

From Eqs. (148) and (150) we compute the moments of $\delta T_{n}$ to first order in $\delta s=L_{0} / l$,

$$
\begin{aligned}
\frac{1}{\delta s}\left\langle\delta T_{n}\right\rangle= & -T_{n}+\frac{2 T_{n}}{\beta N+2-\beta} \\
& \times\left(1-T_{n}+\frac{\beta}{2} \sum_{m(\neq n)} \frac{T_{n}+T_{m}-2 T_{n} T_{m}}{T_{n}-T_{m}}\right), \\
\frac{1}{\delta s}\left\langle\delta T_{n} \delta T_{m}\right\rangle= & \delta_{n m} \frac{4 T_{n}^{2}\left(1-T_{n}\right)}{\beta N+2-\beta} .
\end{aligned}
$$

The third and higher moments vanish to first order in $\delta s$. It follows from the theory of Brownian motion (Van Kampen, 1981) that the probability distribution $P\left(T_{1}, T_{2}, \ldots, T_{N}, s\right)$ of the transmission eigenvalues evolves with increasing $s=L / l$ according to the FokkerPlanck equation ${ }^{17}$

\footnotetext{
${ }^{16}$ If $\beta=4$ the quaternion matrices $U$ and $V$ are each others dual, $V=U^{\mathrm{R}}$. To apply the formulas of Appendix B, we represent the $N \times N$ quaternion matrix $U$ by a $2 N \times 2 N$ complex matrix $\mathcal{U}$. Then $\langle f(U, V)\rangle=\int d \mu(\mathcal{U}) f\left(\mathcal{U}, \mathcal{C}^{\mathrm{T}} \mathcal{U}^{\mathrm{T}} \mathcal{C}\right)$, where the matrix $\mathcal{C}$ is defined in Eq. (73).

${ }^{17}$ The Itô-Stratonovich ambiguity (Van Kampen, 1981) of Brownian motion with a position-dependent diffusion coefficient does not arise, because Eq. (153) explicitly relates the change in the variables $T_{n}$ to their value prior to the change.
} 
$\frac{\partial P}{\partial S}=\frac{1}{\delta s} \sum_{n=1}^{N} \frac{\partial}{\partial T_{n}}\left(-\left\langle\delta T_{n}\right\rangle P+\frac{1}{2} \sum_{m=1}^{N} \frac{\partial}{\partial T_{m}}\left\langle\delta T_{n} \delta T_{m}\right\rangle P\right)$.

Equation (154) becomes the DMPK equation (145) upon a change of variables from $T_{n}$ to $\lambda_{n}=(1$ $\left.-T_{n}\right) / T_{n}$.

The derivation of the DMPK equation given here rests on the assumption of isotropy of the distribution of scattering matrices. It is possible to replace the isotropy assumption by the weaker assumption of equivalent scattering channels (Mello and Tomsovic, 1991, 1992). This is the assumption that the first two moments of the reflection matrix $r_{0}$ of the thin slice are the same as one would obtain for an isotropic distribution. For example, for $\beta=2$ the requirement of equivalent channels is

$$
\begin{aligned}
& \left\langle\left(r_{0}\right)_{l j}\right\rangle=0, \quad\left\langle\left(r_{0}\right)_{l j}\left(r_{0}\right)_{k l}\right\rangle=0, \\
& \left\langle\left(r_{0}\right)_{l j}\left(r_{0}^{*}\right)_{k l}\right\rangle=N^{-2}\left\langle\operatorname{Tr}\left(1-\mathcal{T}_{0}\right)\right\rangle \delta_{l k} \delta_{l l} .
\end{aligned}
$$

One can see that Eq. (155) is a weaker assumption than the isotropy assumption by considering the case of a thin slice without scattering. Then $r_{0}=0$ and $\mathcal{T}_{0}=1$, so that Eq. (155) is trivially satisfied, but the scattering matrix of the thin slice has a delta-function rather than an isotropic distribution. Dorokhov (1988) has constructed a model of $N$ weakly coupled chains for which the equivalent-channel assumption is exact. This is a special model. More generally, neither the isotropy nor the equivalent-channel assumption hold exactly. For example, the transmission probability which follows from the Boltzmann equation with isotropic impurity scattering is about twice as large for normal incidence than it is for grazing incidence (Nieuwenhuizen and Luck, 1993), simply because about half of the electrons at grazing incidence are scattered back before penetrating a meanfree-path deep into the disordered region. [More subtle, quantum-mechanical deviations have been noticed in simulations of the Anderson model by Jalabert and Pichard (1995).] Still, as we will see, the DMPK equation provides a remarkably accurate description of the distribution of the transmission eigenvalues of a disordered wire, on length scales ranging from below the mean free path to above the localization length. Moreover, in the metallic regime the restriction $L \gg W$ to a wire geometry can be relaxed considerably. It is only when the transverse dimension $W$ becomes comparable to the localization length that the DMPK equation breaks down completely.

\section{Mapping to a free-fermion model}

In the absence of time-reversal symmetry, the DMPK equation is equivalent to a Schrödinger equation for $N$ noninteracting fermions in one dimension. This formal correspondence permits an exact solution of the DMPK equation for $\beta=2$ (Beenakker and Rejaei, 1993, 1994a).

To carry out the mapping it is convenient to first write the DMPK equation in terms of a new set of variables $x_{n}$, related to $\lambda_{n}$ and $T_{n}$ by

$$
\lambda_{n}=\sinh ^{2} x_{n}, \quad T_{n}=1 / \cosh ^{2} x_{n}, \quad x_{n} \geqslant 0 .
$$

As discussed in Sec. I.C.1, $\exp \left( \pm 2 x_{n}\right)$ is an eigenvalue of the transfer-matrix product $M M^{\dagger}$. By applying this change of variables to Eq. (145), one finds that the distribution $P\left(x_{1}, x_{2}, \ldots, x_{N}, s\right)$ of the $x_{n}$ 's evolves with increasing $s=L / l$ according to a Fokker-Planck equation with a constant diffusion coefficient,

$$
\begin{aligned}
& \frac{\partial P}{\partial s}=\frac{1}{2 \gamma} \sum_{n=1}^{N} \frac{\partial}{\partial x_{n}}\left(\frac{\partial P}{\partial x_{n}}+\beta P \frac{\partial \Omega}{\partial x_{n}}\right) \\
& \Omega=-\sum_{l=1}^{N} \sum_{j=l+1}^{N} \ln \left|\sinh ^{2} x_{j}-\sinh ^{2} x_{l}\right|-\frac{1}{\beta} \sum_{l=1}^{N} \ln \left|\sinh 2 x_{l}\right|
\end{aligned}
$$

We have abbreviated

$$
\gamma=\beta N+2-\beta \text {. }
$$

The probability distribution $P\left(\left\{x_{n}\right\}, s\right)$ is related to a wave function $\Psi\left(\left\{x_{n}\right\}, s\right)$ by the transformation

$$
P=\Psi e^{-\beta \Omega / 2}
$$

originally introduced by Sutherland (1972) to solve the Fokker-Planck equation of Dyson's Brownian-motion model [given by Eq. (15) for $\beta=2$ ]. Substitution into Eq. (157) yields for $\Psi$ a Schrödinger equation in imaginary time,

$$
\begin{aligned}
-\frac{\partial \Psi}{\partial s} & =\mathcal{H} \Psi, \\
\mathcal{H}= & -\frac{1}{2 \gamma} \sum_{t}\left(\frac{\partial^{2}}{\partial x_{l}^{2}}+\frac{1}{\sinh ^{2} 2 x_{l}}\right) \\
& +\frac{\beta(\beta-2)}{2 \gamma} \sum_{l<l} \frac{\sinh ^{2} 2 x_{j}+\sinh ^{2} 2 x_{l}}{\left(\cosh 2 x_{j}-\cosh 2 x_{l}\right)^{2}} .
\end{aligned}
$$

The Fokker-Planck equation considered by Sutherland maps onto a Hamiltonian with a translationally invariant interaction potential $\left(x-x^{\prime}\right)^{-2}$ (the CalogeroSutherland Hamiltonian, Calogero, 1969; Sutherland, 1971). The interaction potential in Eq. (160) is not translationally invariant. Using a trigonometric identity it can be rewritten as $\sinh ^{-2}\left(x-x^{\prime}\right)+\sinh ^{-2}\left(x+x^{\prime}\right)$, to show that the breaking of translational invariance is due to the interaction between $x$ and an "image charge" at $-x$ '. Caselle (1995) has pointed out that the Hamiltonian (160) belongs to the same family as the CalogeroSutherland Hamiltonian, in the sense that both represent the Laplacian on a certain curved space (first identified by Hüffmann, 1990).

For $\beta=2$ the interaction vanishes identically, reducing $\mathcal{H}$ to a sum of single-particle Hamiltonians $\mathcal{H}_{0}$,

$$
\mathcal{H}_{0}=-\frac{1}{4 N} \frac{\partial^{2}}{\partial x^{2}}-\frac{1}{4 N \sinh ^{2} 2 x} .
$$

The spectrum of $\mathcal{H}_{0}$ is continuous, with eigenvalues $\varepsilon=\frac{1}{4} k^{2} / N$ and eigenfunctions (scattering states)

$$
\psi_{k}(x)=\left[\pi k \tanh \left(\frac{1}{2} \pi k\right) \sinh (2 x)\right]^{1 / 2} \mathrm{P}_{(1 / 2)(k-1)}(\cosh 2 x)
$$


labeled by a wave number $k>0$. (The Legendre functions $\mathrm{P}_{(1 / 2)(\imath k-1)}$ are known as "toroidal functions," because they appear as solutions to the Laplace equation in toroidal coordinates.) An antisymmetric $N$-fermion wave function $\Psi$ can be constructed from a Slater determinant of single-particle scattering states. The transformation

$$
P=\Psi \times \prod_{l<J}\left(\sinh ^{2} x_{j}-\sinh ^{2} x_{l}\right) \prod_{l}\left(\sinh 2 x_{l}\right)^{1 / 2}
$$

then yields a symmetric probability distribution $P$.

We conclude that the Brownian motion of $N$ transmission eigenvalues in the absence of time-reversal symmetry is equivalent to a scattering problem of $N$ noninteracting fermions in one dimension. The correlations due to eigenvalue repulsion are fully accounted for by the requirement of an antisymmetric $N$-fermion wave function. An exact solution for $P$ can be written down for arbitrary initial conditions. For the application to an ensemble of disordered wires we need the ballistic initial condition ${ }^{18}$

$$
\lim _{s \rightarrow 0} P\left(\left\{x_{n}\right\}, s\right)=\prod_{\imath=1}^{N} \delta\left(x_{\imath}-0^{+}\right),
$$

which says that all $T_{n}$ 's are equal to 1 if $L \ll l$. The exact solution of the DMPK equation (145) with $\beta=2$ for the initial condition (164) is (Beenakker and Rejaei, 1993, 1994a)

$$
\begin{aligned}
P= & C(s) \prod_{l<j}\left(\sinh ^{2} x_{j}-\sinh ^{2} x_{l}\right) \prod_{l} \sinh 2 x_{l} \\
& \times \operatorname{Det}\left[\int_{0}^{\infty} d k e^{-k^{2} s / 4 N} \tanh \left(\frac{1}{2} \pi k\right) k^{2 m-1}\right. \\
& \left.\times \mathrm{P}_{(1 / 2)(l k-1)}\left(\cosh 2 x_{n}\right)\right] .
\end{aligned}
$$

Here $C(s)$ is an $x$-independent normalization factor and Det $a_{n m}$ denotes the determinant of the matrix with elements $a_{n m}(1 \leqslant n, m \leqslant N)$. For $N=1$, Eq. (165) reduces to the $\beta$-independent solution of the scaling equation (146) for a 1D chain (first obtained by Gertsenshtein and Vasil'ev, 1959). Equation (165) generalizes the 1Dchain solution to arbitrary $N$, for the case $\beta=2$.

In the presence of time-reversal symmetry, for $\beta=1$ or 4 , there exists no exact solution of the DMPK equation in terms of functions of a single variable [such as the Legendre functions in Eq. (165)]. The interaction potential in the Hamiltonian (160) leads to correlations between the $x_{n}$ 's which cannot be represented by a Slater determinant. Caselle (1995) has shown that these correlations are described by functions of $N$ variables known in the mathematical literature as "zonal spherical func-

\footnotetext{
${ }^{18}$ Other initial conditions have been considered by Beenakker and Melsen (1994), see Sec. III.D.1, and by Frahm and Müller-Groeling (1996), to compute the correlator $\langle G(s) G(s+\Delta s)\rangle$.
}

tions." It is only for $\beta=2$ that these functions factorize into functions of a single variable. For $\beta=1$ or 4 , an expression for $P$ in terms of functions of a single variable can be obtained in the metallic regime $s \ll N$ (Sec. III.B.5) and also in the insulating regime $s \gg N$ (Sec. III.C.1) - but not in the crossover regime.

\section{Equivalence to a supersymmetric field theory}

A field theory for localization in disordered wires has been developed by Efetov and Larkin (1983), building on work by Wegner (1979). (For reviews, see Efetov, 1983, 1996.) The diffusion modes are represented by matrix fields $Q$ containing an equal number of commuting and anticommuting elements. By analogy with the supersymmetry between bosons and fermions in particle physics, such matrices are called supersymmetric matrices, or supermatrices. The interaction between the diffusion modes is described by a model known in quantum field theory as the nonlinear $\sigma$ model (Itzykson and Zuber, 1980). The adjective nonlinear refers to the constraint $Q^{2}=1$, and the letter $\sigma$ originates from an early notation for the fields. The restriction to a wire geometry makes the fields one-dimensional (1D) in the spatial coordinate. It has been demonstrated by Brouwer and Frahm (1996) (following up on a paper by Rejaei, 1996) that the $1 \mathrm{D} \sigma$ model is equivalent to the thick-wire limit of the DMPK equation. The thick-wire limit is defined by $N \rightarrow \infty, L / l \rightarrow \infty$ at constant ratio $N l / L$. The equivalence holds for an arbitrary $p$-point correlation function of the transmission eigenvalues. Here we give an outline of the equivalence proof for the simplest case $p=1$. It is then sufficient to consider $8 \times 8$ supermatrices in the $\sigma$ model.

The quantity which relates the $1 \mathrm{D} \sigma$ model to the DMPK equation is the generating function $F\left(\theta_{1}, \theta_{2}, \theta_{3}, \theta_{4} ; T_{1}, T_{2}, \ldots, T_{N}\right)$, defined by

$$
\begin{aligned}
F\left(\left\{\theta_{l}\right\} ;\left\{T_{n}\right\}\right)= & \prod_{n=1}^{N}\left(\frac{1-T_{n} \sin ^{2}\left[\frac{1}{2}\left(\theta_{3}+\theta_{4}\right)\right]}{1+T_{n} \sinh ^{2}\left[\frac{1}{2}\left(\theta_{1}+\theta_{2}\right)\right]}\right. \\
& \left.\times \frac{1-T_{n} \sin ^{2}\left[\frac{1}{2}\left(\theta_{3}-\theta_{4}\right)\right]}{1+T_{n} \sinh ^{2}\left[\frac{1}{2}\left(\theta_{1}-\theta_{2}\right)\right]}\right)^{(1 / 2)\left(1+\delta_{\beta, 4}\right)},
\end{aligned}
$$

$\theta_{2} \equiv 0$ if $\beta \in\{2,4\} ; \quad \theta_{4} \equiv 0$ if $\beta \in\{1,2\}$.

The doubling of the exponent in Eq. (166a) for $\beta=4$ originates from Kramers' degeneracy of the transmission eigenvalues $T_{n}$. (The product over $n$ runs only over the $N$ distinct eigenvalues.) The function $F$ is called a generating function because its ensemble average yields the density $\rho(T)$ of the transmission eigenvalues,

$$
\rho(T)=-(\pi T \sqrt{1-T})^{-1} \operatorname{Re} \frac{\partial}{\partial \theta_{3}}\langle F\rangle,
$$

evaluated at $\theta_{2}=\theta_{4}=0, \quad \sin ^{2} \frac{1}{2} \theta_{3}=-\sinh ^{2} \frac{1}{2} \theta_{1}=(T$ $\left.+i 0^{+}\right)^{-1}$. The angles $\theta_{t}$ parametrize the supermatrices of the nonlinear $\sigma$ model. There are three independent 
$\theta_{l}$ 's if $\beta=1,4$, and two if $\beta=2$. For example, if $\beta=2$ the parametrization is (Efetov, 1983, 1996)

$$
Q=\left(\begin{array}{ll}
u & 0 \\
0 & v
\end{array}\right)\left(\begin{array}{cc}
\cos \hat{\theta} & i \sin \hat{\theta} \\
-i \sin \hat{\theta} & -\cos \hat{\theta}
\end{array}\right)\left(\begin{array}{cc}
u^{-1} & 0 \\
0 & v^{-1}
\end{array}\right) .
$$

Here $u$ and $v$ are $4 \times 4$ unitary supermatrices and $\hat{\theta}$ is a $4 \times 4$ diagonal matrix with elements $\theta_{3}, \theta_{3}, i \theta_{1}, i \theta_{1}$ on the diagonal. The variable $\theta_{1} \in(0, \infty)$ is called a noncompact angle, and $\theta_{3} \in(0, \pi)$ is called a compact angle. A similar parametrization of $Q$ exists for $\beta=1$ (with two noncompact angles $\theta_{1}, \theta_{2}$ and one compact angle $\theta_{3}$ ), and for $\beta=4$ (with two compact angles $\theta_{3}, \theta_{4}$ and one noncompact angle $\theta_{1}$ ).

A remarkable property of the function $F$ is that the DMPK equation yields a closed evolution equation for its ensemble average (Brouwer and Frahm, 1996):

$$
l \frac{\partial\langle F\rangle}{\partial L}=\frac{\beta\left(1+\delta_{\beta, 4}\right)^{-1}}{\beta N+2-\beta} \sum_{l} \frac{1}{J_{\theta}} \frac{\partial}{\partial \theta_{l}} J_{\theta} \frac{\partial}{\partial \theta_{l}}\langle F\rangle .
$$

The sum over $i$ runs over the two $(\beta=2)$ or three $(\beta=1,4)$ independent angles. The factor $J_{\theta}$ is the Jacobian from the space of supermatrices $Q$ to the space of angles $\theta_{t}$, given by

$$
J_{\theta}= \begin{cases}\frac{\sinh \theta_{1} \sinh \theta_{2} \sin ^{3} \theta_{3}}{\Pi_{\sigma_{1}, \sigma_{2}= \pm 1} \sinh ^{2}\left[\frac{1}{2}\left(\theta_{1}+\sigma_{1} \theta_{2}+i \sigma_{2} \theta_{3}\right)\right]} & \text { if } \beta=1, \\ \frac{\sinh \theta_{1} \sin \theta_{3}}{\Pi_{\sigma_{1}= \pm 1} \sinh ^{2}\left[\frac{1}{2}\left(\theta_{1}+i \sigma_{1} \theta_{3}\right)\right]} & \text { if } \beta=2, \\ \frac{\sinh ^{3} \theta_{1} \sin \theta_{3} \sin \theta_{4}}{\Pi_{\sigma_{1}, \sigma_{2}= \pm 1} \sinh ^{2}\left[\frac{1}{2}\left(\theta_{1}+i \sigma_{1} \theta_{3}+i \sigma_{2} \theta_{4}\right)\right]} & \text { if } \beta=4 .\end{cases}
$$

The practical importance of Eq. (169) is that it allows one to compute the ensemble average of $F$ (and hence the eigenvalue density) by solving a partial differential equation involving only two or three variables-in contrast to the $N$ variables in the DMPK equation (145). [A similar method exists for Dyson's Brownian-motion model (Guhr, 1996).] The conceptual importance of Eq. (169) is that (for $N \gg 1$ ) the same evolution equation is obtained if one computes $\langle F\rangle$ from the $1 D \sigma$ model. This was shown by Rejaei (1996; for $\beta=2)$ and by Brouwer and Frahm (1996; for $\beta=1,4)$. The conclusion is that $\rho(T)$ is the same whether computed from the 1D $\sigma$ model or from the DMPK equation. This equivalence can be generalized to all $p$-point correlation functions. It holds for arbitrary $N l / L$ if the same initial conditions are chosen in both descriptions but requires the thickwire limit $N \gg 1$ (since the $\sigma$ model can only be formulated in this limit).

The $1 \mathrm{D} \sigma$ model has been derived from three microscopic descriptions of the conductor. Efetov and Larkin (1983) started from a homogeneous wire with a whitenoise potential. Iida, Weidenmüller, and Zuk (1990a, 1990b) studied a chain of disordered grains, each grain having a random Hamiltonian drawn from the Gaussian ensemble. Fyodorov and Mirlin (1991, 1994) considered a tight-binding Hamiltonian, whose nonzero elements lie in a band around the diagonal (a so-called banded random matrix). Because of the equivalence discussed above, each of these three models can also be considered as being a microscopic model for the DMPK equation.

\section{B. Metallic regime}

\section{Conductance}

In the metallic regime, for wire lengths $L$ much less than the localization length $N l$, the conductance is known to decrease linearly with $L$ (Ohm's law). Let us verify that the DMPK equation correctly describes this classical scaling for $N l / L \gg 1$.

We use the method of moments of Mello and Stone (Mello, 1988; Mello and Stone, 1991). This is a method for computing the moments of

$$
M_{q}=\sum_{n=1}^{N} T_{n}^{q}, \quad q=1,2, \ldots,
$$

as an expansion in inverse powers of $N$. From the DMPK equation (145) one derives a hierarchy of coupled evolution equations for moments of $M_{q}$. For example, the evolution of $M_{1}, M_{2}$, and $M_{3}$ is coupled by the equation

$$
\begin{aligned}
\frac{\partial}{\partial s}\left\langle M_{1}^{p}\right\rangle= & \frac{-p \beta}{\beta N+2-\beta}\left\langle M_{1}^{p+1}-(1-2 / \beta) M_{1}^{p-1} M_{2}\right. \\
& \left.-2(p-1) \beta^{-1} M_{1}^{p-2}\left(M_{2}-M_{3}\right)\right\rangle .
\end{aligned}
$$

The hierarchy closes order by order in the large- $N$ expansion. Indeed, since $M_{q}^{p}=\mathcal{O}\left(N^{p}\right)$, Eq. (172) reduces to

$$
\frac{\partial}{\partial s}\left\langle M_{1}^{p}\right\rangle=-p N^{-1}\left\langle M_{1}^{p+1}\right\rangle+\mathcal{O}\left(N^{p-1}\right),
$$

to leading order in $N$. Notice that the symmetry index $\beta$ has dropped out in this order. The ballistic initial condition $\left(T_{n}=1\right.$ for all $n$ if $\left.s=0\right)$ implies

$$
\lim _{s \rightarrow 0}\left\langle M_{1}^{p}\right\rangle=N^{p}
$$

Equation (173), with $p=1,2, \ldots$, forms a recursive set of differential equations. The solution with initial condition (174) is

$$
\left\langle M_{1}^{p}\right\rangle=N^{p}(1+s)^{-p}+\mathcal{O}\left(N^{p-1}\right) .
$$

In view of the Landauer formula (33), the average conductance is $\langle G\rangle=G_{0}\left\langle M_{1}\right\rangle$ (with $G_{0}=2 e^{2} / h$ ), hence

$$
\left\langle G / G_{0}\right\rangle=N(1+s)^{-1}+\mathcal{O}\left(N^{0}\right) .
$$

In the diffusive limit $(s=L / l \gg 1)$ the conductance decreases linearly with $L,\left\langle G / G_{0}\right\rangle \rightarrow N l / L$, as expected. Furthermore, comparison with the Drude formula (Ashcroft and Mermin, 1976) shows that the mean free path $l$ of the scaling theory [defined in Eq. (151)] is related to the transport mean free path $l_{\mathrm{tr}}$ of kinetic theory by the numerical coefficient given in Eq. (152). In the ballistic 
limit $(s \ll 1)$ the conductance $\left\langle G / G_{0}\right\rangle \rightarrow N$ reaches the contact conductance of an $N$-mode wire between wide reservoirs. The crossover from the ballistic to the diffusive limit, for $s \simeq 1$, is not described exactly by Eq. (176), but the error is small [about 3\% for isotropic impurity scattering (De Jong, 1994)].

By carrying out the expansion of the moments to order $N^{0}$, one can compute the weak-localization correction $\delta G=\langle G\rangle-G_{0}(1+s)^{-1}$ to the average conductance given by Eq. (176), as well as the variance Var $G=\left\langle G^{2}\right\rangle-\langle G\rangle^{2}$. The results are (Mello, 1988)

$$
\begin{aligned}
& \delta G / G_{0}=\frac{1}{3}(1-2 / \beta) \frac{s^{3}}{(1+s)^{3}}+\mathcal{O}\left(N^{-1}\right), \\
& \operatorname{Var} G / G_{0}=\frac{2}{15} \beta^{-1}\left(1-\frac{1+6 s}{(1+s)^{6}}\right)+\mathcal{O}\left(N^{-1}\right) .
\end{aligned}
$$

The diffusive limits $s \rightarrow \infty$ of Eqs. (177) and (178),

$$
\delta G / G_{0} \rightarrow \frac{1}{3}(1-2 / \beta), \quad \text { Var } G / G_{0} \rightarrow \frac{2}{15} \beta^{-1},
$$

agree precisely with diagrammatic perturbation theory (Lee and Stone, 1985; Mello and Stone, 1991).

The method of moments can in principle be applied to all polynomial linear statistics, i.e., transport properties of the form $A=\Sigma_{n} a\left(T_{n}\right)$ with $a(T)$ a polynomial in $T$. It is an efficient way to compute the mean and variance of the conductance [for which $a(T)=T$ ], since only a few levels of the hierarchy of evolution equations have to be considered. With a great deal of effort it is possible to apply the method of moments to the shot-noise power (De Jong and Beenakker, 1992), for which $a(T)=T-T^{2}$. Other transport properties, for which $a(T)$ is not a polynomial, require the more general method discussed in the next subsection.

In experiments on disordered wires, phase coherence is in general not maintained throughout the whole wire length. The numerical coefficients in Eq. (179) are therefore much larger than measured (for a review, see Beenakker and Van Houten, 1991). The $\beta$ dependence is insensitive, however, to phase-breaking processes. Moreover, while the numerical coefficients are specific for a wire geometry, the $\beta$ dependence is the same in wires, thin films, and bulk samples. In the absence of spin-orbit scattering, application of a magnetic field induces a $\beta=1 \rightarrow \beta=2$ transition, leading to an increase of the average conductance and a reduction of the variance by a factor of two. Measurements by Mailly and Sanquer (1992) of this symmetry-class transition are shown in Fig. 16. The typical field scale for the transition is one flux quantum through a phase coherent region. ${ }^{19}$ In the presence of strong spin-orbit scattering, a magnetic field induces a $\beta=4 \rightarrow \beta=2$ transition, leading to a decrease of the average conductance. The change in $\beta$ is accompanied by a removal of Kramers' degeneracy of the

\footnotetext{
${ }^{19}$ At much higher fields (when the Zeeman energy becomes larger than the Thouless energy), a further reduction of the variance by a factor of two takes place, associated with the removal of spin degeneracy (measured by Debray et al., 1989).
}

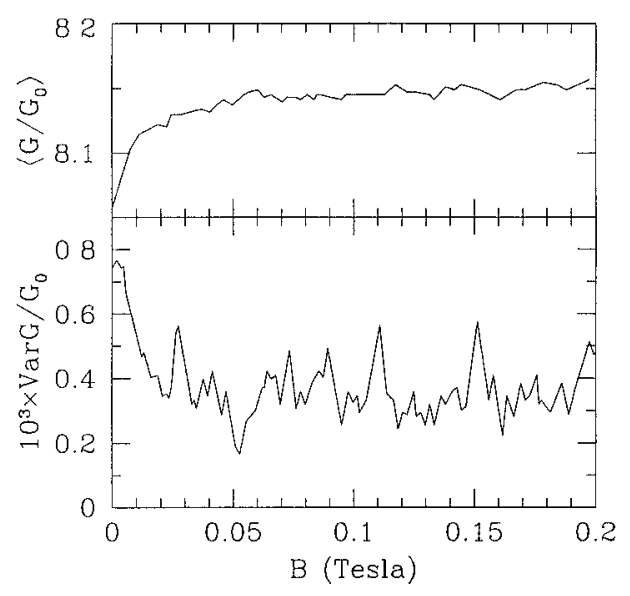

FIG. 16. Mean and variance of the conductance as a function of magnetic field, measured by averaging over 50 impurity configurations in a single Si-doped $\mathrm{GaAs}$ wire $(T=45 \mathrm{mK}$, $W=0.09 \mu \mathrm{m}, L=10 \mu \mathrm{m})$. Uncorrelated impurity configurations were generated by thermal cycling to room temperature. A short phase-coherence length $l_{\phi} \leqslant L$ reduces the zero-field weak-localization correction and variance to $\delta G / G_{0}=-l_{\phi} / L$, $\operatorname{Var} G / G_{0}=3\left(l_{\phi} / L\right)^{3}$. Comparing with the data we estimate $l_{\phi} \approx 0.65 \mu \mathrm{m}$. The $\beta=1 \rightarrow \beta=2$ transition leads to an increase of the average conductance and to a halving of the variance, as observed in the experiment. After Mailly and Sanquer (1992).

transmission eigenvalues. The net result is that $\operatorname{Var} G$ is reduced by a factor of two, just as in the absence of spin-orbit scattering (Altshuler and Shklovskiř, 1986; measured by Birge, Golding, and Haemmerle, 1989; Millo et al., 1990).

\section{Other transport properties}

To compute the mean and variance of arbitrary linear statistics $A=\Sigma_{n} a\left(x_{n}\right)$, one needs the density of transmission eigenvalues

$$
\rho(x)=\left\langle\sum_{i} \delta\left(x-x_{i}\right)\right\rangle
$$

and the two-point correlation function

$$
K\left(x, x^{\prime}\right)=\left\langle\sum_{i, j} \delta\left(x-x_{i}\right) \delta\left(x^{\prime}-x_{j}\right)\right\rangle-\rho(x) \rho\left(x^{\prime}\right) .
$$

(We recall the parametrization $T_{n}=1 / \cosh ^{2} x_{n}, x_{n} \geqslant 0$.) In the metallic regime it is sufficient to know $\rho$ and $K$ to order $N^{0}$. Dorokhov (1984) and Mello and Pichard (1989) computed the leading-order term in $\rho$ (which is of order $N$ ), while Beenakker (1994b) and Macêdo and Chalker (1994) computed the next term (of order $N^{0}$ ). The leading-order term in $K$ (which is of order $N^{0}$ ) was computed by Chalker and Macêdo (1993) and by Beenakker and Rejaei (1993). The derivations are given in the following subsections. The results are (in the diffusive limit $L \gg l$ )

$$
\rho(x)=\frac{N l}{L}+\left(1-\frac{2}{\beta}\right)\left[\frac{1}{4} \delta\left(x-0^{+}\right)+\frac{1}{4 x^{2}+\pi^{2}}\right],
$$




$$
\begin{aligned}
& K\left(x, x^{\prime}\right)=\mathcal{K}\left(x-x^{\prime}\right)+\mathcal{K}\left(x+x^{\prime}\right), \\
& \mathcal{K}(x)=-\frac{1}{2 \beta \pi^{2}} \frac{d^{2}}{d x^{2}} \ln \left[1+(\pi / x)^{2}\right] .
\end{aligned}
$$

The density $\rho(x)$ has a cutoff at $x \simeq L / l$, such that $\int_{0}^{\infty} d x \rho(x)=N$. Since only the range $x \leqq 1$ contributes to transport properties, this large- $x$ cutoff need not be specified more accurately. The integrable singularities at $x=0$ in both $\rho$ and $\mathcal{K}$ are in reality smeared out over a few eigenvalue spacings. Equations (182) and (183) are sufficiently accurate if $a(x)$ is smooth on the scale $\delta x$ of the eigenvalue spacing. Since $\delta x \simeq L / N l \ll 1$ in the metallic regime, this is not a strong requirement.

The mean and variance of $A=\Sigma_{n} a\left(x_{n}\right)$ follow upon integration,

$$
\begin{aligned}
\langle A\rangle= & \frac{N l}{L} \int_{0}^{\infty} d x a(x)+\left(1-\frac{2}{\beta}\right) \\
& \times\left[\frac{1}{4} a(0)+\int_{0}^{\infty} d x \frac{a(x)}{4 x^{2}+\pi^{2}}\right] \\
\operatorname{Var} A= & \frac{1}{2 \beta \pi^{2}} \int_{0}^{\infty} d x \int_{0}^{\infty} d x^{\prime}\left(\frac{d a(x)}{d x}\right) \\
& \times\left(\frac{d a\left(x^{\prime}\right)}{d x^{\prime}}\right) \ln \left(\frac{1+\pi^{2}\left(x-x^{\prime}\right)^{-2}}{1+\pi^{2}\left(x+x^{\prime}\right)^{-2}}\right)
\end{aligned}
$$

The double integral in Eq. (185a) reduces to a single integral if the Fourier transform $a(k)$ $=2 \int_{0}^{\infty} d x a(x) \cos k x$ is known,

$$
\operatorname{Var} A=\frac{1}{2 \beta \pi^{2}} \int_{0}^{\infty} d k\left(1-\mathrm{e}^{-\pi k}\right) k|a(k)|^{2} .
$$

The first term in Eq. (184) is the semiclassical value of $A$, which is of order $N$ and $\beta$ independent. The second term is the weak-localization correction, which is of order $N^{0}$ and has a $1-2 / \beta$ dependence on the symmetry index. The variance (185) has no order- $N$ contribution (universality). The leading-order term is of order $N^{0}$ and is inversely proportional to $\beta$.

Equations (184) and (185) reduce the computation of $\langle A\rangle$ and $\operatorname{Var} A$ to a quadrature, regardless of the complexity of the function $a(x)$. Let us check these formulas for the case that $A$ is the conductance, $G / G_{0}=\Sigma_{n} 1 / \cosh ^{2} x_{n}$. Substitution of $a(x)=1 / \cosh ^{2} x$ into Eq. (184) yields $\left\langle G / G_{0}\right\rangle=N l / L+\frac{1}{3}(1-2 / \beta)$, in agreement with Eqs. (176) and (177) in the diffusive limit $(s \gg 1)$. Similarly, substitution of the Fourier transform $a(k)=\pi k / \sinh \left(\frac{1}{2} \pi k\right)$ into Eq. (185b) yields $\operatorname{Var} G / G_{0}=\frac{2}{15} \beta^{-1}$, in agreement with Eq. (178).

\section{Transmission eigenvalue density}

The derivation that we present of the eigenvalue density in the metallic regime is based on Mello and Pichard (1989) for the $\mathcal{O}(N)$ term and Beenakker (1994b) for the $\mathcal{O}\left(N^{0}\right)$ correction. The starting point is the DMPK equation (145) for the probability distribution $P\left(\left\{\lambda_{n}\right\}, s\right)$ of the $\lambda$ variables. We seek to reduce it to an equation for the density $\rho(\lambda, s)=\left\langle\Sigma_{n} \delta\left(\lambda-\lambda_{n}\right)\right\rangle$. Multiplying both sides of Eq. (145) by $\Sigma_{n} \delta\left(\lambda-\lambda_{n}\right)$ and integrating over $\lambda_{1}, \lambda_{2}, \ldots, \lambda_{N}$, one obtains

$$
\begin{aligned}
& \frac{\partial \rho}{\partial s}=\frac{2}{\gamma} \frac{\partial}{\partial \lambda} \lambda(1+\lambda)\left(\frac{\partial \rho}{\partial \lambda}-\beta I\right), \\
& I(\lambda, s)=\int_{0}^{\infty} \frac{d \lambda^{\prime}}{\lambda-\lambda^{\prime}}\left(\sum_{l \neq j} \delta\left(\lambda-\lambda_{l}\right) \delta\left(\lambda^{\prime}-\lambda_{\jmath}\right)\right) .
\end{aligned}
$$

(Recall the definition $\gamma=\beta N+2-\beta$.) The integral over the pair distribution function has the large- $N$ expansion (Dyson, 1972; see Appendix A for a derivation)

$$
\frac{I(\lambda, s)}{\rho(\lambda, s)}=\int_{0}^{\infty} d \lambda^{\prime} \frac{\rho\left(\lambda^{\prime}, s\right)}{\lambda-\lambda^{\prime}}+\frac{1}{2} \frac{\partial}{\partial \lambda} \ln \rho(\lambda, s)+\mathcal{O}\left(N^{-1}\right) .
$$

Substitution into Eq. (186) gives a nonlinear evolution equation for the eigenvalue density,

$$
\begin{aligned}
\frac{\partial \rho}{\partial s}= & \frac{1}{\gamma} \frac{\partial}{\partial \lambda} \lambda(1+\lambda) \rho \frac{\partial}{\partial \lambda}((2-\beta) \ln \rho \\
& \left.-2 \beta \int_{0}^{\infty} d \lambda^{\prime} \rho\left(\lambda^{\prime}, s\right) \ln \left|\lambda-\lambda^{\prime}\right|\right) .
\end{aligned}
$$

At this point it is convenient to switch from the $\lambda$ to the $x$ variables (defined by $\lambda_{n}=\sinh ^{2} x_{n}$ ). The densities are related by $\rho(x, s)=\rho(\lambda, s) d \lambda / d x$. In terms of the $x$ variables, Eq. (188) takes the form

$$
\begin{aligned}
\frac{\partial \rho}{\partial s}= & \frac{1}{4 \gamma} \frac{\partial}{\partial x} \rho \frac{\partial}{\partial x}[(2-\beta)(\ln \rho-\ln |\sinh 2 x|) \\
& \left.-2 \beta \int_{0}^{\infty} d x^{\prime} \rho\left(x^{\prime}, s\right) \ln \left|\sinh ^{2} x-\sinh ^{2} x^{\prime}\right|\right]
\end{aligned}
$$

We need to solve Eq. (189) to the same order in $N$ as the expansion (187), i.e., neglecting terms of order $N^{-1}$. To this end we decompose $\rho=\rho_{0}+\delta \rho$, with $\rho_{0}$ of order $N$ and $\delta \rho$ of order $N^{0}$. Substitution into Eq. (189) yields to order $N$ an equation for $\rho_{0}$,

$$
\frac{\partial \rho_{0}}{\partial s}=-\frac{1}{2 N} \frac{\partial}{\partial x} \rho_{0} \frac{\partial}{\partial x} \int_{0}^{\infty} d x^{\prime} \rho_{0}\left(x^{\prime}, s\right) \ln \left|\sinh ^{2} x-\sinh ^{2} x^{\prime}\right|
$$

It is possible to solve this evolution equation for all $s$, as we will discuss in the next subsection. Here we only need the solution in the diffusive limit $s \gg 1$. Then the $x$ variables have the uniform density

$$
\rho_{0}(x, s)=\left\{\begin{array}{l}
N / s \text { if } x \leqslant s, \\
0 \text { if } x \geqslant s
\end{array}\right.
$$

as one can verify by substitution into Eq. (190).

Linearization of Eq. (189) around $\rho_{0}$ yields an equation for $\delta \rho$, 


$$
\begin{aligned}
& \frac{1}{2} \frac{d^{2}}{d x^{2}} \int_{0}^{\infty} d x^{\prime} \delta \rho\left(x^{\prime}\right) \ln \left|\sinh ^{2} x-\sinh ^{2} x^{\prime}\right|+\frac{d}{d x}(x \delta \rho) \\
& \quad=\frac{1}{4}\left(1-\frac{2}{\beta}\right) \frac{d^{2}}{d x^{2}} \ln |\sinh 2 x| .
\end{aligned}
$$

The $s$ dependence has dropped out in the limit $s \gg 1$. The integro-differential equation (192) can be solved by means of the identity

$$
\begin{aligned}
& \int_{0}^{\infty} d x^{\prime} f\left(x^{\prime}\right) \ln \left|\sinh ^{2} x-\sinh ^{2} x^{\prime}\right| \\
& \quad=\int_{-\infty}^{\infty} d x^{\prime} f\left(\left|x^{\prime}\right|\right) \ln \left|\sinh \left(x-x^{\prime}\right)\right|,
\end{aligned}
$$

which simplifies the integration to a convolution. The Fourier transform of $\delta \rho(x)$ then satisfies an ordinary differential equation, which is easily solved. The result is

$$
\delta \rho(x)=(1-2 / \beta)\left[\frac{1}{4} \delta\left(x-0^{+}\right)+\left(4 x^{2}+\pi^{2}\right)^{-1}\right] .
$$

The correction (194) to the uniform density (191) takes the form of a deficit (for $\beta=1$ ) or an excess (for $\beta=4$ ), concentrated in the region $x \leqslant 1$. Equations (191) and (194) together form Eq. (182), which was used in the previous subsection.

\section{Scaling as a hydrodynamic flow}

The uniform eigenvalue density (191) is the large-s limit of the solution $\rho_{0}(x, s)$ of the evolution equation (190). Let us investigate how this limit is reached starting from an initially nonuniform density. It turns out that the nonlinear integro-differential equation (190) can be solved exactly for arbitrary initial condition (Beenakker, Rejaei, and Melsen, 1994). The solution is based on a mapping of Eq. (190) onto Euler's equation of hydrodynamics. A similar mapping exists for Dyson's Brownian-motion model (Pandey and Shukla, 1991). For notational simplicity we will write $\rho$ instead of $\rho_{0}$ in this subsection, being only concerned here with the leading-order contribution in powers of $N$.

We begin by rewriting Eq. (190) in terms of the $\lambda$ variables,

$$
\frac{\partial \rho}{\partial s}=-\frac{2}{N} \frac{\partial}{\partial \lambda} \lambda(1+\lambda) \rho \frac{\partial}{\partial \lambda} \int_{0}^{\infty} d \lambda^{\prime} \rho\left(\lambda^{\prime}, s\right) \ln \left|\lambda-\lambda^{\prime}\right| .
$$

The density $\rho(\lambda, s)$ has the Stieltjes transform

$$
F(z, s)=\int_{0}^{\infty} d \lambda^{\prime} \frac{\rho\left(\lambda^{\prime}, s\right)}{z-\lambda^{\prime}} .
$$

The function $F(z, s)$ is an analytic function of $z$ in the complex plane cut by the positive real axis, which vanishes for large $|z|$ as

$$
\lim _{|z| \rightarrow \infty} F(z, s)=N / z
$$

It has a discontinuity for $z=\lambda \pm i 0^{+}(\lambda>0)$. The limiting values $F_{ \pm}(\lambda, s) \equiv F\left(\lambda \pm i 0^{+}, s\right)$ are

$$
F_{ \pm}= \pm \frac{\pi}{i} \rho(\lambda, s)+\frac{\partial}{\partial \lambda} \int_{0}^{\infty} d \lambda^{\prime} \rho\left(\lambda^{\prime}, s\right) \ln \left|\lambda-\lambda^{\prime}\right| .
$$

Combination of Eqs. (195) and (198) gives

$$
N \frac{\partial}{\partial s}\left(F_{+}-F_{-}\right)=-\frac{\partial}{\partial \lambda} \lambda(1+\lambda)\left(F_{+}^{2}-F_{-}^{2}\right),
$$

which implies that the function

$$
\mathcal{F}(z, s)=N \frac{\partial}{\partial s} F(z, s)+\frac{\partial}{\partial z} z(1+z) F^{2}(z, s)
$$

is analytic in the whole complex plane, including the real axis. Moreover, $\mathcal{F} \rightarrow 0$ for $|z| \rightarrow \infty$, in view of Eq. (197). We conclude that $\mathcal{F} \equiv 0$, since the only analytic function that vanishes at infinity is identically zero.

We now return from the $\lambda$ to the $x$ variables. The mapping $z=\sinh ^{2} \zeta$ maps the $z$ plane onto the strip in the $\zeta$ plane between the lines $y=0$ and $y=-\pi / 2$, where $\zeta=x+i y$. The mapping is conformal if we cut the $z$ plane by the two half lines $\lambda>0$ and $\lambda<-1$ on the real axis. On this strip we define the auxiliary function $U=U_{x}+i U_{y}$ by

$$
U(\zeta, s) \equiv \frac{F}{2 N} \frac{d z}{d \zeta}=\frac{\sinh 2 \zeta}{2 N} \int_{0}^{\infty} d x^{\prime} \frac{\rho\left(x^{\prime}, s\right)}{\sinh ^{2} \zeta-\sinh ^{2} x^{\prime}} .
$$

The equation $\mathcal{F} \equiv 0$ then takes the form

$$
\frac{\partial}{\partial s} U(\zeta, s)+U(\zeta, s) \frac{\partial}{\partial \zeta} U(\zeta, s)=0,
$$

which we recognize as Euler's equation of hydrodynamics: $\left(U_{x}, U_{y}\right)$ is the velocity field in the $(x, y)$ plane of a two-dimensional ideal fluid at constant pressure. Euler's equation is easily solved. For initial condition $U(\zeta, 0)=U_{0}(\zeta)$ the solution to Eq. (202) is

$$
U(\zeta, s)=U_{0}(\zeta-s U(\zeta, s))
$$

From $U$ we obtain the eigenvalue density

$$
\rho(x, s)=(2 N / \pi) \operatorname{Im} U\left(x-i 0^{+}, s\right) .
$$

The ballistic initial condition $\rho(x, 0)=N \delta\left(x-0^{+}\right)$corresponds to $U_{0}(\zeta)=\operatorname{coth} \zeta$. The solution of the implicit equation (203) is plotted in Fig. 17 for several values of $s=L / l$. With increasing disorder the eigenvalue density spreads along the $x$ axis, such that $0<\rho \leqslant N / s$ for $x<x_{\max }$ and $\rho \equiv 0$ for $x \geqslant x_{\max }$. The edge $x_{\max }$ of the density profile is located at

$$
\begin{aligned}
x_{\max } & =\frac{1}{2} \operatorname{arcosh}(1+2 s)+\frac{1}{2} \sqrt{(1+2 s)^{2}-1} \\
& =s+\frac{1}{2} \ln 4 s+\frac{1}{2}+\mathcal{O}(1 / s),
\end{aligned}
$$

with $\rho \propto \sqrt{x_{\max }-x}$ for $x$ close to $x_{\max }$. For $s \gg 1$ the density tends to the diffusive limit (191) of a step-function profile: $\rho(x, s) \rightarrow N / s$ for $s \rightarrow \infty$ at fixed $x / s<1$. In fact, this limit is reached regardless of the particular initial condition because of the fixed point $\zeta=-\frac{1}{2} i \pi$, at which $U=0 \quad$ [see Eq. (201)]. To see this, define $\zeta_{0}=\zeta-s U(\zeta, s)$ and write Eq. (203) in the form $\zeta-\zeta_{0}=s U_{0}\left(\zeta_{0}\right)$. For $s \rightarrow \infty, \zeta_{0} \rightarrow-\frac{1}{2} i \pi$ so that the prod- 


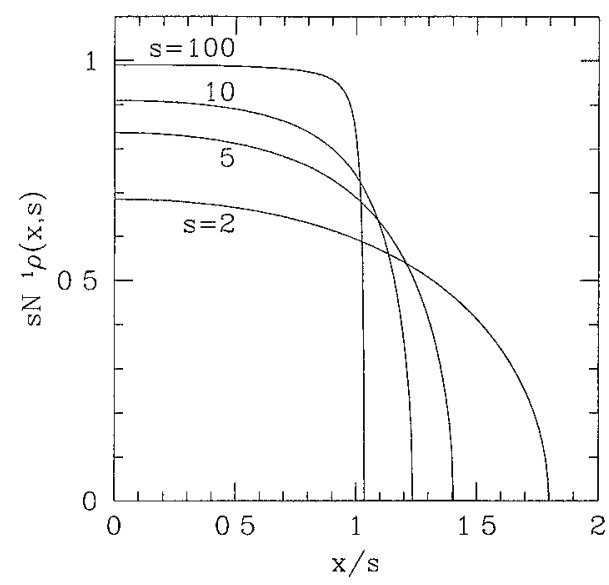

FIG. 17. Eigenvalue density in the metallic regime. The variable $x$ is related to the transmission eigenvalue by $T=1 / \cosh ^{2} x$. Curves are computed from Eq. (203) for four values of $s=L / l$, with the ballistic initial condition for the eigenvalue density $\rho(x, 0)=N \delta\left(x-0^{+}\right)$. For $s \gg 1$ the density tends to the limit (191) of a step-function profile.

uct $s U_{0}\left(\zeta_{0}\right)$ remains finite. It follows that $U(\zeta, s) \rightarrow\left(\zeta+\frac{1}{2} i \pi\right) / s$, hence $\rho(x, s) \rightarrow N / s$.

\section{Nonlogarithmic eigenvalue repulsion}

The tiny difference between the variance of the conductance in a quantum dot and in a disordered wire has a fundamental implication for the repulsion of the transmission eigenvalues (see Sec. I.D.2). Since a logarithmic repulsion of the $\lambda$ variables implies that $\beta \operatorname{Var} G / G_{0}$ $=\frac{1}{8}$, while in a wire one has $\beta \operatorname{Var} G / G_{0}=\frac{2}{15}$, it follows that the repulsion of the $\lambda$ 's cannot be precisely logarithmic (Beenakker, 1993a, 1993c). Recall that a logarithmic repulsion follows from the Jacobian from matrix to eigenvalue space. A nonlogarithmic repulsion means that there exists correlations between the eigenvalues that do not have a geometric origin. To determine the eigenvalue repulsion in a disordered wire in the metallic regime, we consider the exact solution (165) of the DMPK equation for $\beta=2$.

If $1 \ll s \ll N$ the dominant contribution to the integral over $k$ in Eq. (165) comes from the range $k \geq(N / s)^{1 / 2} \gg 1$. In this range $\tanh \left(\frac{1}{2} \pi k\right) \rightarrow 1$ and the Legendre function simplifies to a Bessel function,

$$
\mathrm{P}_{(1 / 2)(l k-1)}(\cosh 2 x) \rightarrow J_{0}(k x)(2 x / \sinh 2 x)^{1 / 2} .
$$

The $k$ integration can now be carried out analytically,

$$
\begin{aligned}
& \int_{0}^{\infty} d k e^{-k^{2} s / 4 N} k^{2 m-1} J_{0}\left(k x_{n}\right) \\
& \quad=\frac{1}{2}(m-1) !(4 N / s)^{m} e^{-x_{n}^{2} N / s} L_{m-1}\left(x_{n}^{2} N / s\right),
\end{aligned}
$$

with $L_{m-1}$ a Laguerre polynomial. We then apply the determinantal identity

$$
\text { Det } L_{m-1}\left(x_{n}^{2} N / s\right)=c \operatorname{Det}\left(x_{n}^{2}\right)^{m-1}=c \prod_{l<j}\left(x_{j}^{2}-x_{\imath}^{2}\right),
$$

with $c$ an $x$-independent number [which can be absorbed in $C(s)]$. The first equality in Eq. (208) holds because the determinant of a matrix is unchanged if any one column of the matrix is added to any other column, so that we can reduce the Laguerre polynomial in $x^{2}$ of degree $m-1$ to just its highest-order term $x^{2(m-1)}$ times a numerical coefficient. The second equality expands the Vandermonde determinant.

Collecting results, we find that the solution (165) of the DMPK equation for $\beta=2$ simplifies in the metallic regime to (Beenakker and Rejaei, 1993, 1994a)

$$
\begin{aligned}
P= & C(s) \prod_{l<J}\left[\left(\sinh ^{2} x_{J}-\sinh ^{2} x_{l}\right)\left(x_{J}^{2}-x_{l}^{2}\right)\right] \\
& \times \prod_{l}\left[\exp \left(-x_{l}^{2} N / s\right)\left(x_{l} \sinh 2 x_{l}\right)^{1 / 2}\right] .
\end{aligned}
$$

Caselle (1995) has generalized Eq. (209) to $\beta=1$ and 4. The result for the three values of $\beta$ can be written in the form of a Gibbs distribution,

$$
\begin{aligned}
& P=C(s) \exp \left[-\beta\left(\sum_{l<j} u\left(x_{l}, x_{j}\right)+\sum_{l} V\left(x_{l}\right)\right)\right] \\
& u\left(x_{l}, x_{J}\right)=-\frac{1}{2} \ln \left|\sinh ^{2} x_{j}-\sinh ^{2} x_{l}\right|-\frac{1}{2} \ln \left|x_{j}^{2}-x_{l}^{2}\right| \\
& V(x)=\frac{1}{2}(\gamma / s) \beta^{-1} x^{2}-\frac{1}{2} \beta^{-1} \ln |x \sinh 2 x| .
\end{aligned}
$$

In terms of the $\lambda$ variables $\left(\lambda=\sinh ^{2} x\right)$, the interaction takes the form

$u\left(\lambda_{l}, \lambda_{j}\right)=-\frac{1}{2} \ln \left|\operatorname{arsinh}^{2} \lambda_{J}^{1 / 2}-\operatorname{arsinh}^{2} \lambda_{l}^{1 / 2}\right|-\frac{1}{2} \ln \left|\lambda_{J}-\lambda_{l}\right|$.

For $\lambda \ll 1$ [i.e., for $T=(1+\lambda)^{-1}$ close to unity] $u\left(\lambda_{l}, \lambda_{J}\right) \rightarrow-\ln \left|\lambda_{J}-\lambda_{l}\right|$, so we obtain a logarithmic repulsion for the strongly transmitting scattering channels. However, for $\lambda \approx 1$ the interaction (211) is nonlogarithmic. For fixed $\lambda_{l} \ll 1, u\left(\lambda_{l}, \lambda_{J}\right)$ as a function of $\lambda_{j}$ crosses over from $-\ln \left|\lambda_{J}-\lambda_{t}\right|$ to $-\frac{1}{2} \ln \left|\lambda_{J}-\lambda_{t}\right|$ as $\lambda_{J} \rightarrow \infty$ (see Fig. 18). We conclude that, for weakly transmitting channels, the interaction is twice as small as predicted by considerations based solely on the Jacobian.

The reduced level repulsion for weakly transmitting channels enhances the variance of the conductance above the result $\beta$ Var $G / G_{0}=\frac{1}{8}$ for a purely logarithmic repulsion. To see this, we compute the two-point correlation function $K\left(x, x^{\prime}\right)$ from the interaction potential $u\left(x, x^{\prime}\right)$, using the property that $K$ and $u$ are each others functional inverse in the large- $N$ limit (Beenakker, 1993a, 1993c; see Sec. I.D.1):

$$
\beta \int_{0}^{\infty} d x^{\prime \prime} u\left(x, x^{\prime \prime}\right) K\left(x^{\prime \prime}, x^{\prime}\right)=\delta\left(x-x^{\prime}\right), \quad x, x^{\prime}>0 .
$$

To solve this integral equation, we note that the decomposition of the interaction potential (210b) into

$$
\begin{aligned}
& u\left(x, x^{\prime}\right)=\mathcal{U}\left(x-x^{\prime}\right)+\mathcal{U}\left(x+x^{\prime}\right)+\text { constant, } \\
& \mathcal{U}(x)=-\frac{1}{2} \ln |2 x \sinh x|
\end{aligned}
$$




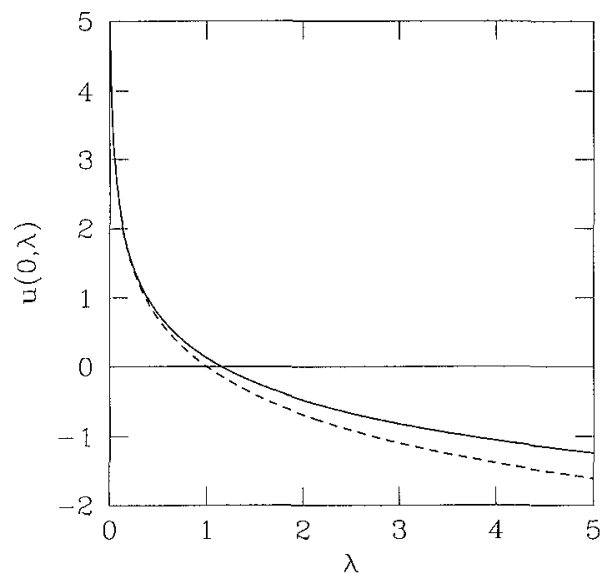

FIG. 18. Eigenvalue interaction potential $u\left(\lambda_{i}, \lambda_{j}\right)$ for $\lambda_{i}=0$ as a function of $\lambda_{1} \equiv \lambda$. The solid curve is the result (211) from the DMPK equation in the metallic regime. The dashed curve is the logarithmic repulsion $-\ln |\lambda|$ dictated by the Jacobian from matrix to eigenvalue space. For $\lambda \ll 1$ the two curves coincide. For $\lambda \rightarrow \infty$ their ratio approaches a factor of two. After Beenakker and Rejaei (1994a).

implies for the two-point correlation function the decomposition

$$
K\left(x, x^{\prime}\right)=\mathcal{K}\left(x-x^{\prime}\right)+\mathcal{K}\left(x+x^{\prime}\right) .
$$

[The additive constant in Eq. (213a) is irrelevant because of the sum rule $\int_{0}^{\infty} d x^{\prime \prime} K\left(x^{\prime \prime}, x^{\prime}\right)=0$ implied by the definition (181) of $K$.] The functions $\mathcal{U}(x)$ and $\mathcal{K}(x)$, defined for both positive and negative $x$, are related by the convolution

$\beta \int_{-\infty}^{\infty} d x^{\prime \prime} \mathcal{U}\left(x-x^{\prime \prime}\right) \mathcal{K}\left(x^{\prime \prime}-x^{\prime}\right)=\delta\left(x-x^{\prime}\right)+$ constant

which is readily inverted by Fourier transformation:

$$
\mathcal{K}(k)=\frac{|k|}{\beta \pi}\left(1-e^{-\pi|k|}\right) .
$$

Transforming back from $k$ to $x$ one finds the two-point correlation function (183) used in Sec. III.B.2 to compute the variance of the conductance. The result $\beta$ Var $G / G_{0}=\frac{2}{15}$ is only slightly larger than the value $\frac{1}{8}$ for a logarithmic repulsion, because only the weakly transmitting channels (which contribute little to the conductance) are affected by the nonlogarithmic interaction.

The derivation of the two-point correlation function presented here emphasizes the relationship with the eigenvalue interaction (Beenakker and Rejaei, 1993, 1994a; Caselle, 1995). There exists an alternative derivation (Chalker and Macêdo, 1993; Macêdo and Chalker, 1994), which starts directly from the DMPK equation and reduces it to an evolution equation for $K$ in the metallic regime. The diffusive limit $s \rightarrow \infty$ then leads to Eq. (183). We discussed a similar approach in Sec. III.B.3, in connection with the eigenvalue density. It is worthwhile to check that the result of Eq. (194) for $\delta \rho$ obtained there agrees with the density implied by the distribution (210). This alternative route to the eigenvalue density is described in Appendix C.

\section{Localized regime}

\section{Log-normal distribution of the conductance}

In the metallic regime, the root-mean-square fluctuations of the conductance are a factor $L / N l$ smaller than the average conductance. The sample-to-sample fluctuations are therefore relatively unimportant, since $L \ll N l$. As the length $L$ of the wire increases beyond the localization length $\xi \simeq N l$, the localized regime is entered. Then fluctuations become as large as the average, which is no longer representative for the conductance of a single sample. The conductance distribution $P(G)$, which was well approximated by a Gaussian in the metallic regime, ${ }^{20}$ becomes very broad and asymmetric, with a peak at small $G$ and a long tail towards large $G$.

It follows from general properties of products of random matrices that $P(G)$ is log-normal in the limit $L / N l \rightarrow \infty$, that is to say, $\ln G$ has a Gaussian distribution (Imry, 1986a). To see this, note that the scaling operation of Fig. 15 corresponds to the multiplication of transfer matrices: $M=\Pi_{i} M_{i}$, where $M_{i}$ is the transfer matrix of segment $i$ and $M$ is the transfer matrix of the entire wire. The limit $L \rightarrow \infty$, at fixed $N$ and $l$, corresponds to the multiplication of an infinite number of random matrices, drawn independently from the same ensemble. In this limit, the $2 N$ random eigenvalues $\exp \left( \pm 2 x_{n}\right)$ of $M M^{\dagger}$ tend to the nonrandom values $\exp \left( \pm 2 L / \xi_{n}\right)$, with $\xi_{n}$ independent of $L$ (Pichard and Sarma, 1981; Pichard and André, 1986). This is known as the "multiplicative ergodic theorem" (Oseledec, 1968; Crisanti, Paladin, and Vulpiani, 1993). The inverse localization lengths $1 / \xi_{n}$ are referred to as the Lyapunov exponents of the random-matrix product. For large but finite $L$, the $x_{n}$ 's have small Gaussian fluctuations around their asymptotic limit $L / \xi_{n}$. The conductance $G=G_{0} \Sigma_{n} \cosh ^{-2} x_{n} \approx 4 G_{0} \exp \left(-2 x_{1}\right)$ is dominated by the smallest $x_{n}$, say $x_{1}$. The conclusion is that $-\frac{1}{2} \ln \left(G / 4 G_{0}\right)$ has the same Gaussian distribution as $x_{1}$.

The mean and variance of the log-normal distribution of the conductance follow directly from the DMPK equation (157) for the probability distribution $P\left(x_{1}, x_{2}, \ldots, x_{N}, s\right)$ (Dorokhov, 1982, 1983; Pichard, 1991). In the limit $L / N l \equiv S / N \rightarrow \infty$ the variables $x_{1} \ll x_{2} \ll \ldots \ll x_{N}$ become widely separated and $\gg 1$, so that the term $\Omega$ in Eq. (157) may be approximated by

$$
\Omega \approx-2 \beta^{-1} \sum_{n=1}^{N}(1+\beta n-\beta) x_{n}+\text { constant. }
$$

The solution of Eq. (157) then factorizes into a product of Gaussians,

\footnotetext{
${ }^{20}$ The third cumulant of $G$ is of order $(L / N l)^{2}\left(e^{2} / h\right)^{3}$ (Macêdo, 1994a; Gopar, Martínez, and Mello, 1995).
} 


$$
\begin{aligned}
& P \approx\left(\frac{\gamma l}{2 \pi L}\right)^{N / 2} \prod_{n-1}^{N} \exp \left[-\frac{\gamma l}{2 L}\left(x_{n}-L / \xi_{n}\right)^{2}\right], \\
& \xi_{n}=\gamma l(1+\beta n-\beta)^{-1}
\end{aligned}
$$

The root-mean-square fluctuation of the $x_{n}$ 's equals $\sqrt{L / \gamma l}$, which is indeed much smaller than their spacing $\beta L / \gamma l$ The conductance is dominated by $x_{1}$, which has a mean $L / \gamma l$ equal to its varıance The Gaussian distribution of $-\ln \left(G / G_{0}\right) \approx 2 x_{1}+\mathcal{O}(1)$ therefore has a mean that is half its variance,

$$
-\left\langle\ln \left(G / G_{0}\right)\right\rangle=\frac{1}{2} \operatorname{Var}\left[\ln \left(G / G_{0}\right)\right]=2 L / \gamma l
$$

The localization length $\xi$ is obtained from the exponential decay of the typical conductance, by identifying $\exp \left\langle\ln \left(G / G_{0}\right)\right\rangle \equiv \exp (-2 L / \xi)$ Hence

$$
\xi=\gamma l=(\beta N+2-\beta) l
$$

The average conductance $\langle G\rangle$ decays more slowly than the typical conductance $\exp \langle\ln G\rangle$

$$
\begin{aligned}
\left\langle G / G_{0}\right\rangle & \propto \int_{0}^{\infty} d x e^{-2 x} \exp \left[-\frac{\gamma l}{2 L}(x-L / \gamma l)^{2}\right] \\
& \propto \exp (-L / 2 \xi)
\end{aligned}
$$

For $N \gg 1$ the localization length $\xi \approx \beta N l$ becomes proportional to the symmetry index $\beta$ This $\beta$ dependence can be measured by studying the effect of a magnetic field on the conductance (Pichard, Sanquer, et al 1990) In the absence of spin-orbit scattering, a timereversal symmetry-breaking magnetic field induces a transition from $\beta=1$ to $\beta=2$, and hence a doubling of $\xi$ The spin degeneracy of the $N$ scattering channels is not broken In the case of strong spin-orbit scattering, breaking of time-reversal symmetry induces a transition from $\beta=4$ to $\beta=2$ and also breaks Kramers' degeneracy of the scattering channels The combined result of $\beta=4 \rightarrow \beta=2$ and $N \rightarrow 2 N$ is that $\xi$ remains unchanged (Efetov and Larkin, 1983, the role of Kramers' degeneracy has been emphasized by Mrrlin, 1994) To observe the doubling of $\xi$ induced by a magnetic field in the absence of spin-orbit scattering requires field strengths $B \geq h / e \xi^{2}$ In weaker fields the magnetoconductance is dominated by thermally actıvated processes (Mott hopping), which leads to an increase of $G$ with $B$ both in the absence and presence of spin-orbit scattering (Nguyen, Spivak, and Shklovskĭ, 1985, Meir et al 1991, Meir and Entın-Wohlman, 1993, for reviews, see Shklovskır and Spivak, 1990, Imry, 1995) It is this positive magnetoconductance that is usually measured in insulators ${ }^{21}$ The simple and universal $\beta$ dependence of the localization length [Eq (220)] is special for a wire geometry (quasione-dimensional sample) and has not yet been observed

\footnotetext{
${ }^{21}$ An exception is formed by the experiments reported by P1chard, Sanquer, et al (1990) on an insulating amorphous Y/S1 alloy with strong spin orbit scattering, which show a negative magnetoconductance ( $G$ decreases with $B$ ) It is not clear how to reconcile this with a theory that properly accounts for Kramers' degeneracy
}

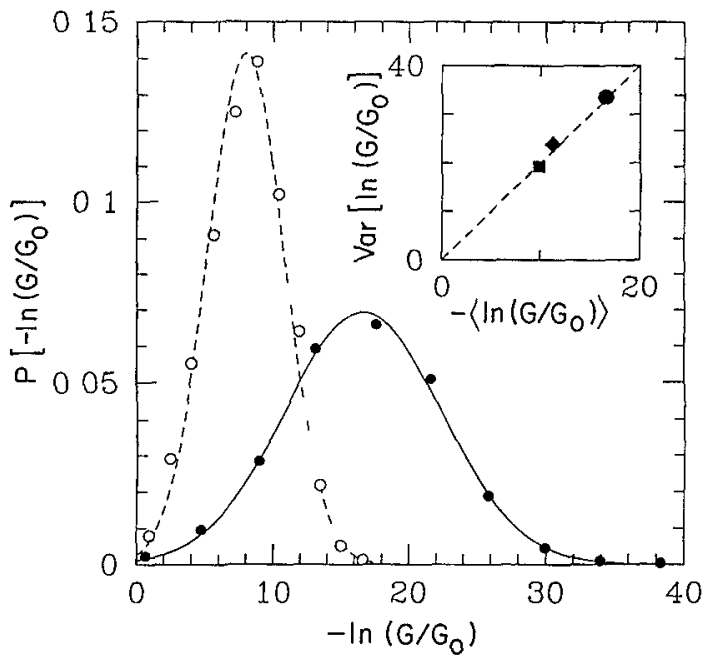

FIG 19 Distribution of $-\ln \left(G / G_{0}\right)$ from a numerical simulation of the Anderson model on a $10 \times 250$ strip (filled dots) and a $10 \times 10$ square (open dots), in zero magnetic field The dashed and solid curves are Gaussians with variance equal to the mean and to twice the mean, respectively The results for a strip are as expected from the Dorokhov-Mello-PereyraKumar equation, those for a square have no known explana tion The inset shows the mean and variance for the strip for three values of the magnetic field circle, diamond, and square correspond, respectively, to $B=0,3 \times 10^{-4}$, and $3 \times 10^{-2} \times h / e a^{2}$ The localization length $\xi=-2 L /\left\langle\ln \left(G / G_{0}\right)\right\rangle$ increases by a factor close to the value $20 / 11$ predicted by $\mathrm{Eq}$ (220) for the $\beta=1 \rightarrow \beta=2$ transition with $N=10$ The dashed line indicates the predicted factor of two between mean and variance After Pichard (1991)

experimentally In two- and three-dimensional samples the $B$ dependence of $\xi$ is more complicated and not untversal (Lerner and Imry, 1995)

The log-normal distribution of the conductance in the localized regime has been verified in numerical simulations of the Anderson model (Pichard, 1991) The disordered region is modeled by a tight-binding Hamiltonian on a two-dimensional square lattice (lattice constant $a$, width $W$, length $L$ ), with a constant hopping term $U_{0}=\hbar^{2} / 2 m a^{2}$ between neighboring sites and with a random impurity potential at each site (uniformly distributed between $\pm \frac{1}{2} U_{\mathrm{d}}$ ) The Fermi level is chosen at the center of the tight-binding band $\left(4 U_{0}\right.$ from the band bottom), at which the number $N$ of propagating modes equals the number W/a of sites in a row (for hard-wall boundary conditions at the two ends of the row) Results for a $10 \times 250$ strip are shown in Fig 19 (filled dots) The disorder is sufficiently strong $\left(U_{\mathrm{d}}=3 U_{0}\right)$ that the wire is deep in the localized regime $(L \approx 8 \xi)$ The distribution of $-\ln \left(G / G_{0}\right)$ is well fitted by a Gaussian (solid curve), with a variance equal to twice the mean The inset shows that a magnetic field significantly increases the localization length (there is no spin-orbit scattering in the simulation) At $B=003 \mathrm{~h} / e \mathrm{a}^{2}$ the increase by a factor of 17 1s close to the factor $20 / 11 \approx 18$ predicted by $\mathrm{Eq}(220)$ for $N=10$ Note also that the factor of two between mean and variance is observed to be $B$ independent, as 
expected. The open dots and dashed curve in Fig. 19 show a still unexplained feature of two-dimensional insulators. The simulation of a $10 \times 10$ square with $U_{\mathrm{d}}=12 U_{0}$ yields a log-normal distribution of the conductance with a variance equal to the mean - not twice the mean as in a quasi-one-dimensional insulator.

\section{Crystallization of transmission eigenvalues}

The exponential decay with increasing wire length of the conductance in the localized regime is associated with a "crystallization" of the transmission eigenvalues (Muttalib, 1990; Pichard, Zanon, et al. 1990; Stone, et al. 1991). In the limit $L / N l \rightarrow \infty$, the $x_{n}$ 's form a onedimensional lattice with spacing $\delta x=L / N l$ (for $N \gg 1$ ). The fluctuations of the $x_{n}$ 's around their lattice positions grow as $L$ decreases and become comparable to the lattice spacing when $L \simeq N l$. If $L \ll N l$ the density is nearly constant, with small ripples of periodicity $\delta x$, reminiscent of a liquid. This is the metallic regime, in which the conductance scales linearly with $L$.

The transition from a liquidlike to a crystallike eigenvalue density can be obtained from the exact solution (165) of the DMPK equation for $\beta=2$, by integrating out $N-1$ of the $x_{n}$ 's:

$$
\rho(x, s)=N \int_{0}^{\infty} d x_{2} \ldots \int_{0}^{\infty} d x_{N} P\left(x, x_{2}, \ldots, x_{N}, s\right) .
$$

The calculation was carried out by Frahm (1995a), using a generalization of the method of orthogonal polynomials suggested by Muttalib (1995). (The same result was obtained from the 1D $\sigma$ model by Rejaei, 1996.) The idea is to write the probability distribution as the product of two determinants,

$$
P=\left(\text { Det } a_{m}\left(x_{n}, s\right)\right)\left(\text { Det } b_{m}\left(x_{n}, s\right)\right),
$$

in such a way that the functions $a$ and $b$ are biorthogonal:

$$
\int_{0}^{\infty} d x a_{n}(x, s) b_{m}(x, s)=\delta_{n m}, \quad 1 \leqslant n, m \leqslant N .
$$

Then the integrals in Eq. (222) reduce to a finite series,

$$
\rho(x, s)=\sum_{n=1}^{N} a_{n}(x, s) b_{n}(x, s),
$$

and similar series exist for the correlation functions of the $x_{n}$ 's.

The exact solution (165) is of the form of Eq. (223) [since the term $\Pi_{l<j}$ in Eq. (165) is a Vandermonde determinant], but the functions in the determinants are not bi-orthogonal. Frahm (1995a) constructed a linear combination of these functions such that Eq. (224) is realized:

$$
a_{n}(x, s)=\exp \left[-(2 n-1)^{2} s / 4 N\right] \mathrm{P}_{n-1}(\cosh 2 x),
$$

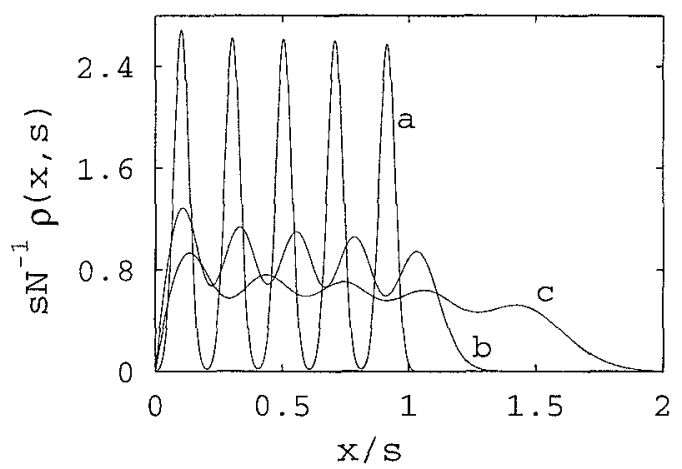

FIG. 20. Exact eigenvalue density for $\beta=2$, computed from Eqs. (225) and (226) for $N=5$ and three values of $s=L / l$ $(s=100,10$, and 2 , respectively, for curves $\mathbf{a}, \mathbf{b}$, and $\mathrm{c})$. The density of the $x_{n}$ 's is not quite uniform in the metallic regime (curve c), because of the relatively small value of $N$ (compare with Fig. 17 for the large- $N$ limit). In the insulating regime the eigenvalues "crystallize" at equally spaced average positions, with small Gaussian fluctuations around the average (curve a). $\rho(x, s)$ is the eigenvalue density, and $x$ is related to the transmission eigenvalue $T$ by $T=1 / \cosh ^{2} x$. After Frahm (1995a).

$$
\begin{aligned}
b_{n}(x, s)= & \frac{1}{2} \sinh 2 x \int_{0}^{\infty} d k e^{-k^{2} s / 4 N} k \tanh \left(\frac{1}{2} \pi k\right) \\
& \times Q_{n}\left(k^{2}\right) \mathrm{P}_{(1 / 2)(i k-1)}(\cosh 2 x),
\end{aligned}
$$

where $\mathrm{P}_{n}$ is a Legendre polynomial and $Q_{n}$ is the interpolation polynomial of Lagrange,

$$
Q_{n}\left(k^{2}\right)=\prod_{p=1(p \neq n)}^{N} \frac{k^{2}+(2 p-1)^{2}}{(2 p-1)^{2}-(2 n-1)^{2}} .
$$

The resulting eigenvalue density is plotted in Fig. 20 , for $N=5$ and values of $s$ in the metallic, crossover, and insulating regimes. One recognizes the appearance of deep minima in the density due to eigenvalue repulsion, upon entering the localized regime.

This nonperturbative result for the density of transmission eigenvalues is for the case $\beta=2$ of broken timereversal symmetry (Frahm, 1995a; Rejaei, 1996). The result for $\beta=1,4$ is not known. What is known nonperturbatively for any $\beta$ is the first and second moment of the conductance. See Zirnbauer (1992) and Mirlin, Müller-Groeling, and Zirnbauer (1994) for the cases $\beta=1,2$, and Brouwer and Frahm (1996) for the case $\beta=4$. Nonperturbative results for entire distributions (rather than moments) exist for the distributions of $\left|t_{n m}\right|^{2}$ and $\Sigma_{n}\left|t_{n m}\right|^{2}$, in the case $\beta=2$ (Van Langen, Brouwer, and Beenakker, 1996). The distribution of the conductance $G / G_{0}=\Sigma_{n, m}\left|t_{n m}\right|^{2}$ is not known exactly for any $\beta$.

\section{Disordered wire with obstacles}

\section{Obstacle as initial condition for scaling}

So far we have concentrated on the DMPK equation with the ballistic initial condition (164). This means that all scattering in the wire is due to disorder, so that for 


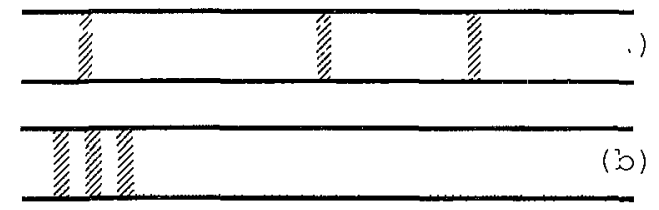

FIG. 21. Two wires containing obstacles (shaded) in series with disordered segments (dotted). For isotropic probability distributions the geometries of (a) and (b) are equivalent. This permits one to treat the effect of the obstacles as an initial condition on the DMPK equation.

$L / l \rightarrow 0$ all transmission eigenvalues $T_{n}$ are equal to 1 . In this subsection we consider the case that the disordered wire contains obstacles, such as point contacts or tunnel barriers, which provide additional scattering. As we will discuss below, if the scattering matrix of each obstacle has an isotropic distribution, then the presence of the obstacles can be accounted for by a nonballistic initial condition on the DMPK equation (Beenakker and Melsen, 1994).

The wire geometry we have in mind is sketched in Fig. 21(a). Disordered segments (dotted) alternate with obstacles (shaded). The disordered segments have $N$ propagating modes, mean free path $l$, and a total length $L$. We model the scattering from the impurities and obstacles by independent and isotropic transfer matrices. That is to say, we write the transfer matrix $M$ of the whole system as the product $M=\Pi_{l} M_{l}$ of the transfer matrices $M_{l}$ of its segments, and then we assume that the $M_{l}$ 's are distributed according to independent and isotropic distributions $p_{l}\left(M_{i}\right)$. A distribution $p(M)$ is called isotropic if it is only a function of the eigenvalues of $M M^{\dagger}$. Under these assumptions, the geometry of Fig. 21(a) is equivalent to that of Fig. 21(b), where the obstacles are in series with a disordered segment of length $L$. To see this, note that the transfer matrices $M$ and $M^{\prime}$ of Figs. 21(a) and 21(b) differ by a permutation of the $M_{l}$ 's. The probability distribution $p(M)$ is given by

$$
p=p_{1} * p_{2} * p_{3} * \cdots,
$$

where the symbol $*$ denotes a convolution,

$$
p_{\imath} * p_{J}(M)=\int d \mu\left(M_{\jmath}\right) p_{l}\left(M M_{\jmath}^{-1}\right) p_{l}\left(M_{\jmath}\right)
$$

and $d \mu(M)$ is the invariant measure on the group of transfer matrices (Mello, Pereyra, and Kumar, 1988). The probability distribution $p^{\prime}\left(M^{\prime}\right)$ is also given by a convolution of the $p_{l}$ 's, but in a different order. It is a property of isotropic distributions that their convolution does not depend on the order: $p_{t}^{*} p_{l}=p_{l}^{*} p_{t}$ if both $p_{t}$ and $p_{,}$are isotropic. It follows that $p=p^{\prime}$, and hence that the geometries of Figs. 21(a) and 21(b) are equivalent.

The obstacles in Fig. 21(b) form an initial condition on the DMPK equation,

$$
\lim _{L \rightarrow 0} P\left(\lambda_{1}, \lambda_{2}, \ldots, \lambda_{N}, L\right)=P_{0}\left(\lambda_{1}, \lambda_{2}, \ldots, \lambda_{N}\right) .
$$

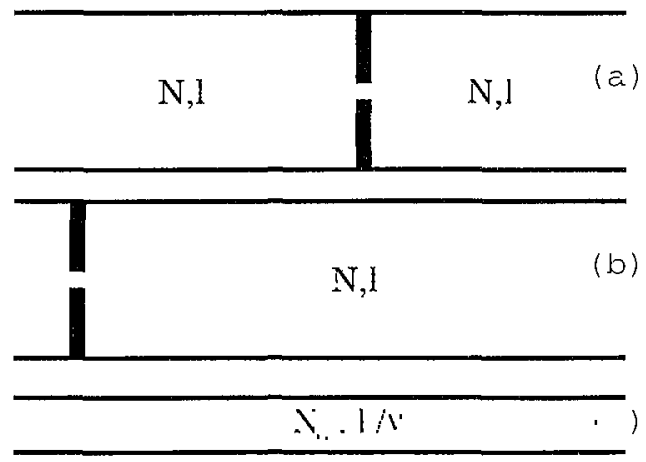

FIG. 22. Equivalence of constricted and unconstricted geometries. (a) Sketch of a ballistic constriction (with conductance $N_{0} G_{0}$ ) in a disordered wire (with mean free path $l$ and $N$ transverse modes). (b) Constricted geometry with all disorder at one side of the point contact, equivalent to (a) for isotropic transfer matrices. (c) Unconstricted geometry, with mean free path $l / \nu$ and $N_{0}$ transverse modes, equivalent to (a) and (b) for $\nu$ given by Eq. (231). After Beenakker and Melsen (1994).

Here $P_{0}$ is the probability distribution of the obstacles in the absence of disordered segments. By solving the DMPK equation with initial condition (230), one determines how disordered segments between the obstacles affect the distribution of the transmission eigenvalues. In the following subsections we will apply this general method to point contacts and tunnel barriers.

\section{Point contact}

As a first application, we consider a disordered wire containing a constriction much narrower than the mean free path (Beenakker and Melsen, 1994). This is known as a ballistic point contact, or a "Sharvin" point contact (Sharvin, 1965). [The opposite limit is known as a diffusive or "Maxwell" point contact (Maxwell, 1891).] The assumption of independent transfer matrices for the constriction and the disordered regions requires a spatial separation of scattering by the impurities and the point contact. This is justified if the mean separation $d_{\text {imp }}$ of the impurities is much greater than the width $W_{0}$ of the constriction. Since $d_{\mathrm{mmp}}$ is much smaller than the mean free path $l$, the condition $d_{1 \mathrm{mp}} \gg W_{0}$ is stronger than the condition $l \gg W_{0}$ for a ballistic point contact. The isotropy assumption for the transfer matrix of the constriction is a simple but realistic model of the coupling between wide and narrow regions, which implies that all $N$ transverse modes in the wide regions (of width $W$ ) to the left and right of the constriction (of width $W_{0}$ ) are equally coupled to each other (Szafer and Stone, 1989). The isotropy assumption for the transfer matrices of the disordered regions (of length $L_{1}$ and $L_{2}$ ) requires aspect ratios $L_{1} / W, L_{2} / W \gg 1$, corresponding to a wire geometry. As argued in the previous subsection, the geometry of Fig. 22(a), with lengths $L_{1}$ and $L_{2}$ of disordered wire to the left and right of the point contact, is equivalent to the geometry of Fig. 22(b), with a length $L=L_{1}+L_{2}$ of disordered wire to one side only. We will now argue that the constricted geometry of Fig. 22(b) is, 
in turn, equivalent to the unconstricted geometry of Fig. 22(c), consisting of a disordered wire with $N_{0}$ transverse modes and mean free path $l / \nu$. The number $N_{0}$ is determined by the conductance $N_{0}\left(2 e^{2} / h\right)$ of the point contact. The fraction $\nu$ is defined by

$$
\nu=\frac{\beta N_{0}+2-\beta}{\beta N+2-\beta} .
$$

The argument goes as follows. A ballistic point contact has, to a good approximation, $T_{n}=1\left(\lambda_{n}=0\right)$ for $1 \leqslant n \leqslant N_{0}$, and $T_{n}=0\left(\lambda_{n} \rightarrow \infty\right)$ for $N_{0}+1 \leqslant n \leqslant N$. (This is a statement about transmission eigenvalues, not about the transmission probabilities of individual modes, which are all of order $N_{0} / N$.) The initial condition (230) becomes

$$
\lim _{L \rightarrow 0} P=\lim _{\Lambda \rightarrow \infty} \prod_{n=1}^{N_{0}} \delta\left(\lambda_{n}\right) \prod_{n=N_{0}+1}^{N} \delta\left(\lambda_{n}-\Lambda\right) .
$$

The closed channels $N_{0}+1 \leqslant n \leqslant N$ are irrelevant for conduction and can be integrated out. The reduced distribution function $\tilde{P}\left(\lambda_{1}, \lambda_{2}, \ldots, \lambda_{N_{0}}, L\right)$ is defined by

$$
\tilde{P}=\int_{0}^{\infty} d \lambda_{N_{0}+1} \int_{0}^{\infty} d \lambda_{N_{0}+2} \cdots \int_{0}^{\infty} d \lambda_{N} P
$$

and satisfies the following evolution equation plus initial condition [see Eqs. (145) and (232)]:

$$
\begin{aligned}
& \frac{l}{2}(\beta N+2-\beta) \frac{\partial \tilde{P}}{\partial L}=\sum_{n=1}^{N_{0}} \frac{\partial}{\partial \lambda_{n}} \lambda_{n}\left(1+\lambda_{n}\right) \tilde{J} \frac{\partial}{\partial \lambda_{n}} \frac{\tilde{P}}{\tilde{J}} \\
& \tilde{J}=\prod_{i=1}^{N_{0}} \prod_{j=i+1}^{N_{0}}\left|\lambda_{j}-\lambda_{i}\right|^{\beta} \\
& \tilde{P}\left(\lambda_{1}, \lambda_{2}, \ldots \lambda_{N_{0}}, 0\right)=\prod_{n=1}^{N_{0}} \delta\left(\lambda_{n}\right) .
\end{aligned}
$$

We now compare with the unconstricted geometry of Fig. 22(c), with $N_{0}$ modes and mean free path $l / \nu$. The probability distribution $P_{\nu}\left(\lambda_{1}, \lambda_{2}, \ldots, \lambda_{N_{0}}, L\right)$ for this geometry is determined by

$$
\begin{aligned}
\frac{l}{2 \nu}\left(\beta N_{0}+2-\beta\right) \frac{\partial P_{\nu}}{\partial L} & =\sum_{n=1}^{N_{0}} \frac{\partial}{\partial \lambda_{n}} \lambda_{n}\left(1+\lambda_{n}\right) \tilde{J} \frac{\partial}{\partial \lambda_{n}} \frac{P_{\nu}}{\tilde{J}}, \\
P_{\nu}\left(\lambda_{1}, \lambda_{2}, \ldots, \lambda_{N_{0}}, 0\right) & =\prod_{n=1}^{N_{0}} \delta\left(\lambda_{n}\right) .
\end{aligned}
$$

Comparison of Eqs. (234) and (235) shows that $\tilde{P}=P_{\nu}$ if $\nu$ is given by Eq. (231).

The mapping between constricted and unconstricted geometries allows us to obtain the effect of the point contact on the conductance directly from the results for disordered wires in Sec. III.B.1. Let us first assume that the individual conductances of the point contact and of the disordered region are both $\gg G_{0}$, or in other words, that $N_{0}$ and $N / s$ are both $\gg 1$. (Recall the definitions $G_{0}=2 e^{2} / h, s=L / l$.) Substitution of $N \rightarrow N_{0}$ and $s \rightarrow \nu s$

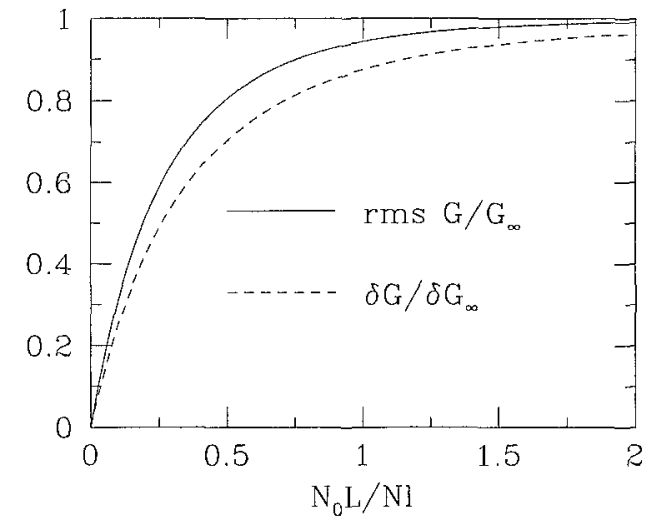

FIG. 23. Suppression by the point contact of the weaklocalization correction $\delta G$ and the root-mean-square conductance fluctuations $\operatorname{rms} G=(\operatorname{Var} G)^{1 / 2}$. The dashed and solid curves are from Eqs. (236) and (237), respectively. [The limit $N_{0} / N \rightarrow 0$ is taken in Eq. (236).] For $N_{0} L / N l \gg 1$ the curves approach the values $\delta G_{\infty}$ and $\mathrm{rms} G_{\infty}$ of an unconstricted disordered wire (normalized to unity in the plot). After Beenakker and Melsen (1994).

in Eqs. (176) and (177) yields the average conductance $\left\langle G / G_{0}\right\rangle=G_{\text {series }}+\delta G$, with $G_{\text {series }}=G_{0}\left(N_{0}^{-1}+s / N\right)^{-1}$ and

$$
\begin{aligned}
\delta G / G_{0}= & (1-2 / \beta)\left[\frac{1}{3}\left(\frac{N_{0} s / N}{1+N_{0} s / N}\right)^{3}\right. \\
& \left.+\left(1-\frac{N_{0}}{N}\right) \frac{N_{0} s / N}{\left(1+N_{0} s / N\right)^{2}}\right] .
\end{aligned}
$$

Similarly, from Eq. (178) one finds the variance

$$
\operatorname{Var} G / G_{0}=\frac{2}{15} \beta^{-1}\left(1-\frac{1+6 N_{0} s / N}{\left(1+N_{0} s / N\right)^{6}}\right) \text {. }
$$

The term $G_{\text {series }}$ is the series addition of the Sharvin conductance $G_{0} N_{0}$ of the ballistic point contact and the Drude conductance $G_{0} N / s$ of the disordered region. The term $\delta G$ is the weak-localization correction to the classical series conductance. This term depends on the ratio $N_{0} s / N$ of the Sharvin and Drude conductances as well as on the ratio $N_{0} / N$ of the width of the point contact and the wide regions. The variance $\operatorname{Var} G$ of the conductance depends only on $N_{0} s / N$. In Fig. 23 we have plotted $\delta G$ and $(\operatorname{Var} G)^{1 / 2}$ as a function of $N_{0} s / N$. (The limit $N_{0} / N \rightarrow 0$ is assumed for $\delta G$.) For large $N_{0} s / N$ the curves tend to $\delta G_{\infty}=\frac{1}{3}(1-2 / \beta) G_{0}$ and $\operatorname{Var} G_{\infty}$ $=\frac{2}{15} \beta^{-1} G_{0}^{2}$, which are the results (179) for weak localization and universal conductance fluctuations in a wire geometry without a point contact. These values are universal to the extent that they are independent of wire length and mean free path. The presence of a point contact breaks this universality, but only if the Sharvin conductance is smaller than the Drude conductance. For $N_{0}>N / s$ the universality is quickly restored. For $N_{0}<N / s$ both $\delta G$ and $\operatorname{Var} G$ are suppressed by the presence of the point contact,

$$
\delta G / G_{0}=(1-2 / \beta) N_{0} s / N+\mathcal{O}\left(N_{0} s / N\right)^{2},
$$




$$
\operatorname{Var} G / G_{0}=2 \beta^{-1}\left(N_{0} s / N\right)^{2}+\mathcal{O}\left(N_{0} s / N\right)^{3},
$$

as first noticed by Maslov, Barnes, and Kirczenow (1993a, 1993b) in a study of the quasiballistic regime $l \gg L$. For a $d$-dimensional point contact, $N_{0} \propto W_{0}^{d-1}$, so that Eq. (239) implies that the root-meansquare fluctuations scale as $\mathrm{rms} G \propto W_{0}^{d-1}$ with the point contact width. A classical argument of series addition of a nonfluctuating contact resistance with a fluctuating background would instead imply the much stronger suppression $\mathrm{rmsG} \propto W_{0}^{2 d-2}$.

Holweg et al. (1991) have measured magnetoconductance fluctuations of a three-dimensional point contact between two $\mathrm{Ag}$ films. The mean free path $l \simeq 200-240 \mathrm{~nm}$ is comparable to the thickness of the metal films and much larger than the diameter $W_{0} \simeq 10-30 \mathrm{~nm}$ of the point contact. The mean separation of the impurities $d_{1 \mathrm{mp}} \simeq\left(l \lambda_{\mathrm{F}}\right)^{1 / 3} \simeq 3 \mathrm{~nm}$ is, however, considerably smaller than $W_{0}$. The root-mean-square conductance fluctuations in the experiment are much larger than predicted by Eq. (239) and moreover scale linearly rather than quadratically with $W_{0}$. Kozub, Caro, and Holweg (1996) have argued that the presence of impurities near the opening of the constriction leads to a substantial enhancement of the conductance fluctuations and to a linear $W_{0}$ dependence, in agreement with their experiment. Possibly, experiments on point contacts in a two-dimensional electron gas (with much larger $\lambda_{F}$ and l) can reach the regime $d_{\text {imp }} \gg W_{0}$, where Eq. (239) is expected to apply. In a numerical simulation of such a system, Maslov, Barnes, and Kirczenow (1993a, 1993b) have indeed obtained results consistent with Eq. (239). ${ }^{22}$

\section{Single-channel limit}

Equations (236) and (237) require $N_{0} \gg 1$, which means that the width $W_{0}$ of the point contact should be much greater than the Fermi wavelength $\lambda_{F}$. Such a point contact is called "classical." A "quantum" point contact has $W_{0}$ comparable to $\lambda_{F}$, so that $N_{0}$ is a small integer. Let us consider the single-channel limit $N_{0}=1$. We assume $N \gg 1$, hence $\nu=2 / \beta N$. The evolution equation for the distribution $\tilde{P}\left(\lambda_{1}, L\right)$ of the single transmitted channel is given by Eq. (146) with a rescaled mean free path $\left(l \rightarrow \frac{1}{2} \beta N l\right)$ and a ballistic initial condition,

$$
\begin{aligned}
& \frac{1}{2} \beta N l \frac{\partial \tilde{P}}{\partial L}=\frac{\partial}{\partial \lambda_{1}} \lambda_{1}\left(1+\lambda_{1}\right) \frac{\partial}{\partial \lambda_{1}} \tilde{P}, \\
& \tilde{P}\left(\lambda_{1}, 0\right)=\delta\left(\lambda_{1}\right) .
\end{aligned}
$$

The solution is (Gertsenshtein and Vasil'ev, 1959; Abrikosov, 1981)

\footnotetext{
${ }^{22}$ Maslov, Barnes, and Kirczenow (1993a, 1993b) consider a geometry as in Fig. 22(a), with $L_{1}=L_{2}=\frac{1}{2} L$, and relate the variance $\operatorname{Var} G$ of the whole system to the variance $\operatorname{Var} G_{1}$ of one of the two disordered segments of length $\frac{1}{2} L$. Their result is $\operatorname{Var} G=\left(N_{0} s / N\right)^{2}\left(l / L_{1}\right)^{2} \operatorname{Var} G_{1}$, in agreement with Eq. (239) [since $\operatorname{Var} G_{1}=2 \beta^{-1}\left(L_{1} / l\right)^{2}$ for $\left.L_{1} \ll l\right]$.
}

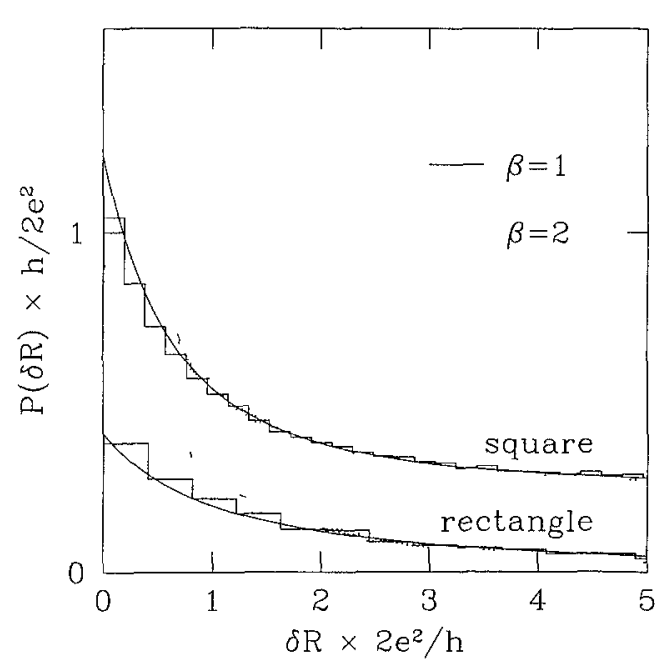

FIG. 24. Probability distribution $P$ of the excess resistance $\delta R=R-h / 2 e^{2}$ of a single-channel point contact in series with a disordered region (square: $N=20, L=W=8.3 l$; rectangle: $N=10, L=2 W=8.3 \mathrm{l}$ ). The histograms are the numerical data (averaged over $10^{4}$ impurity configurations), and the smooth curves are computed from Eq. (241) (with $\delta R=\lambda_{1} h / 2 e^{2}$ ). Solid curves are for zero magnetic field $(\beta=1)$, dotted curves for a flux of $50 \mathrm{~h} / \mathrm{e}$ through the disordered region $(\beta=2)$. For clarity, the curves for the square geometry are offset vertically by 0.25 . After Beenakker and Melsen (1994).

$$
\begin{aligned}
\tilde{P}\left(\lambda_{1}, L\right)= & (2 \pi)^{-1 / 2}(\beta N l / 2 L)^{3 / 2} \exp (-L / 2 \beta N l) \\
& \times \int_{\operatorname{arcosh}\left(1+2 \lambda_{1}\right)}^{\infty} d u \frac{u \exp \left(-u^{2} \beta N l / 8 L\right)}{\left(\cosh u-1-2 \lambda_{1}\right)^{1 / 2}} .
\end{aligned}
$$

[This solution is the single-channel limit of Eq. (165), with an integral representation for the Legendre function.] From Eq. (241) we obtain immediately the distribution $P(\delta R)$ of the excess resistance $\delta R=1 / G-1 / G_{0}=\lambda_{1} / G_{0}$. In the metallic regime $N l / L \gg 1$ the integral over $u$ can be carried out analytically, with the result (Beenakker and Melsen, 1994)

$$
P(\delta R)=\frac{G_{0} \beta N l}{2 L} \exp \left(-\frac{G_{0} \beta N l}{2 L} \delta R\right), \delta R \geqslant 0 .
$$

The width $2 L / \beta N l$ of this exponential distribution decreases by a factor of two upon breaking time-reversal symmetry in the absence of spin-orbit scattering $(\beta=1 \rightarrow \beta=2)$.

A comparison with numerical simulations is shown in Fig. 24 for the Anderson model on a two-dimensional square lattice, as described in Sec. III.C.1. The singlechannel point contact is introduced by assigning a large potential energy to sites at one end of the lattice, so as to create a nearly impenetrable barrier with a narrow opening in the center. The Fermi energy is chosen at $E_{\mathrm{F}}=1.5 U_{0}$ from the band bottom. Two geometries are considered for the wide disordered region: a square geometry ( $L=W=47 a$, corresponding to $N=20$ ), and a rectangular geometry ( $L=2 W=47 a$, corresponding to $N=10)$. The mean free path $l$ which appears in the 


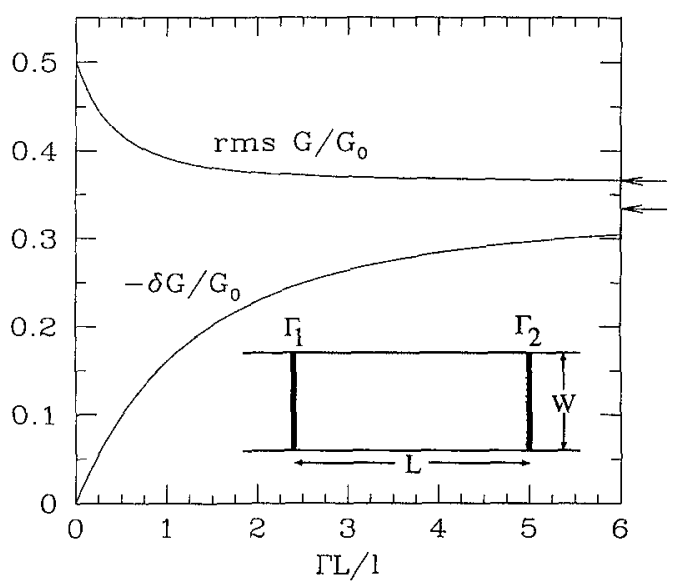

FIG. 25. Weak-localization correction $\delta G$ to the average conductance and root-mean-square fluctuations $\mathrm{rms} G$ (in units of $\left.G_{0}=2 e^{2} / h\right)$, computed from Eqs. (249) and (250) for $\beta=1$. The arrows give the limit $\Gamma L / l \gg 1$. The inset shows the geometry of the double-barrier junction (the disordered region is dotted). The curves plotted in the figure are for a symmetric junction, $\Gamma_{1}=\Gamma_{2} \equiv \Gamma \ll 1$. After Melsen and Beenakker (1995).

DMPK equation is computed numerically from $\operatorname{Tr} t_{\mathrm{d}} t_{\mathrm{d}}^{\dagger}=N(1+L / l)^{-1}$, with $t_{\mathrm{d}}$ the transmission matrix of the wide disordered region without the constriction. [We recall that this mean free path differs by a numerical coefficient from the transport mean free path of kinetic theory, cf. Eqs. (152) and (176).] The results for $P(\delta R)$ plotted in Fig. 24 are for $L / l=8.3$ (disorder strength $\left.U_{\mathrm{d}}=3 U_{0}\right)$. To compare the cases $\beta=1$ and $\beta=2$, the simulations are repeated in the presence of a magnetic flux of $50 \mathrm{~h} / \mathrm{e}$ through the disordered region. The numerical results (histograms) are seen to be in good agreement with the theoretical predictions (smooth curves), without any adjustable parameters. The theory agrees comparably well with the simulations for the square and rectangular geometries, which shows that the condition $L \gg W$ for the validity of the DMPK equation can be relaxed to a considerable extent.

An experimental observation of the exponential distribution of the excess resistance in a quantum point contact is still lacking.

\section{Double-barrier junction}

As a second application, we consider the case that the obstacles in the disordered wire are formed by two tunnel barriers (Melsen and Beenakker, 1995). The geometry is shown in the inset of Fig. 25. A disordered region (length $L$, mean free path $l$, width $W$ ) is separated from ideal $N$-mode leads by two tunnel barriers, with conductances $N \Gamma_{i} G_{0}(i=1,2)$. We assume $N \Gamma_{i} \gg 1$, so that the transmission in the absence of disorder occurs via a large number of overlapping resonances. (For the opposite regime of isolated transmission resonances, see Fertig and Das Sarma, 1989; Leo and MacDonald, 1990; Berkovits and Feng, 1992; Lerner and Raikh, 1992.) Two types of disorder can play a role in a double-barrier junction, interface roughness at the barriers and impurities be- tween the barriers. Interface roughness leads to mesoscopic (sample-to-sample) fluctuations in the conductance even in the absence of any phase coherence, because the tunnel probability $\Gamma$ of a single barrier depends strongly on its thickness. Conductance fluctuations for a single rough tunnel barrier have been studied by Raikh and Ruzin (1991). Here we discuss the case of impurity scattering in the absence of interface roughness. Phase coherence is then essential.

We first assume that the disorder is weak enough that its effect on the average conductance is negligibly small $(l \gg \Gamma L)$, but strong enough to fully mix the transverse modes in the interbarrier region $(l, W \ll L / \Gamma)$. We may then describe the disorder-induced mode mixing by a random $N \times N$ unitary matrix $\Omega$, distributed according to the circular ensemble. The transmission eigenvalues $T_{n}$ are related to the eigenvalues $\exp \left(i \phi_{n}\right)$ of $\Omega$ by

$$
\begin{aligned}
& T_{n}=\left(a+b \cos \phi_{n}\right)^{-1}, \\
& a=\left[1+\left(1-\Gamma_{1}\right)\left(1-\Gamma_{2}\right)\right] / \Gamma_{1} \Gamma_{2}, \\
& b=2 \sqrt{\left(1-\Gamma_{1}\right)\left(1-\Gamma_{2}\right)} / \Gamma_{1} \Gamma_{2} .
\end{aligned}
$$

The statistics of the conductance $G=G_{0} \Sigma_{n} T_{n}$ follows from the probability distribution (67) of the $\phi_{n}$ 's in the circular ensemble.

We seek the average $\langle A\rangle$ and variance $\operatorname{Var} A$ of linear statistics $A=\sum_{n=1}^{N} a\left(\phi_{n}\right)$ on the eigenphases $\phi_{n}$. Since in the circular ensemble the $\phi_{n}$ 's are uniformly distributed in $(0,2 \pi)$, the average is exactly equal to

$$
\langle A\rangle=\frac{N}{2 \pi} \int_{0}^{2 \pi} d \phi a(\phi) .
$$

An exact expression for the variance can also be given (Mehta, 1991) but is cumbersome to evaluate. For $N \gg 1$ we can use a formula analogous to Eq. (58) (Forrester, 1995; Melsen and Beenakker, 1995):

$$
\begin{aligned}
& \operatorname{Var} A=\frac{1}{\beta \pi^{2}} \sum_{n=1}^{\infty} n\left|a_{n}\right|^{2}+\mathcal{O}\left(N^{-1}\right), \\
& a_{n}=\int_{0}^{2 \pi} d \phi e^{i n \phi} a(\phi) .
\end{aligned}
$$

For the conductance we substitute $a(\phi)=(a$ $+b \cos \phi)^{-1}$, with Fourier coefficients $a_{n}=2 \pi\left(a^{2}\right.$ $\left.-b^{2}\right)^{-1 / 2} b^{-n}\left[\left(a^{2}-b^{2}\right)^{1 / 2}-a\right]^{n}$. The results are (assuming $\left.\Gamma_{i} \ll 1\right)$

$$
\begin{aligned}
& \left\langle G / G_{0}\right\rangle=N\left(1 / \Gamma_{1}+1 / \Gamma_{2}\right)^{-1}, \\
& \operatorname{Var} G / G_{0}=\frac{4}{\beta} \frac{\Gamma_{1}^{2} \Gamma_{2}^{2}}{\left(\Gamma_{1}+\Gamma_{2}\right)^{4}} .
\end{aligned}
$$

Equation (246) for the average conductance is what one would expect from classical addition of the resistances $\left(N \Gamma_{i} G_{0}\right)^{-1}$ of the individual barriers. Each member of the ensemble contains a different set of overlapping transmission resonances, and the ensemble average removes any trace of resonant tunneling in $\langle G\rangle$. Equation (247) for the conductance fluctuations tells us that Var $G$ becomes completely independent of $N$ in the 
limit $N \rightarrow \infty$. [More precisely, corrections to Eq. (247) are of order $\left\langle G / G_{0}\right\rangle^{-1}$, which is $\ll 1$ if $N \Gamma_{i} \gg 1$.] The variance reaches a $\Gamma$-independent maximum for two equal barriers: $\operatorname{Var} G / G_{0}=\frac{1}{4} \beta^{-1}$, if $\Gamma_{1}=\Gamma_{2}$. A smaller numerical coefficient $\left(\frac{3}{16}\right.$ instead of $\left.\frac{1}{4}\right)$ has been obtained by Fal'ko (1995), using a different method. The origin of the difference is not yet understood.

We now relax the assumption $l \gg \Gamma L$ to include the case that the impurity scattering is sufficiently strong to affect the average conductance. The $L$ dependence of the distribution of the transmission eigenvalues is governed by the DMPK equation, with the circular ensemble as initial condition. The mean and variance of the conductance can be computed using the method of moments described in Sec. III.B.1. The results for $\Gamma_{1}=\Gamma_{2} \equiv \Gamma \ll 1$ are

$$
\begin{aligned}
& \langle G\rangle=N G_{0}\left(s+1 / \Gamma_{1}+1 / \Gamma_{2}\right)^{-1}+\delta G \\
& \delta G / G_{0}=\frac{1}{3}(1-2 / \beta)-\frac{1-2 / \beta}{(2+\Gamma s)^{3}}\left(\frac{8}{3}+2 \Gamma s\right), \\
& \operatorname{Var} G / G_{0}=\frac{2}{15 \beta}+\frac{4}{\beta(2+\Gamma s)^{6}}\left(\Gamma^{2} s^{2}+\frac{8}{5} \Gamma s+\frac{28}{15}\right) .
\end{aligned}
$$

Equations (249) and (250) are plotted in Fig. 25, for the case $\beta=1$. We see that impurity scattering leads to the appearance of a weak-localization effect on the average conductance. The conductance fluctuations become universal (i.e., independent of $\Gamma$ ) if $L$ exceeds a length $l / \Gamma$, which is parametrically greater than the mean free path. A similar conclusion has been reached by Iida, Weidenmüller, and Zuk (1990a, 1990b), who used the supersymmetry technique to study the conductance fluctuations of a chain of disordered grains as a function of the coupling strength to two electron reservoirs. Their model [which has also been studied by Argaman (1995, 1996) using a semiclassical method] is qualitatively similar but different in detail from the homogeneously disordered conductor considered here (cf. Sec. I.E).

Experimentally, the effects of disorder on tunneling through double-barrier junctions have been studied mainly in semiconductor quantum wells, where the resonances are widely separated because of the small barrier separation $L$ relative to the Fermi wavelength $\lambda_{F}$. Conductance fluctuations of order $e^{2} / h$ in such a structure have been observed by Ghenim et al. (1996). The results presented above apply to the opposite regime of strongly overlapping resonances, relevant to metal structures (where $\lambda_{\mathrm{F}}$ is very short, comparable to the interatomic separation), or to tunneling in the plane of a twodimensional electron gas (where $L$ can be quite long, because of the large phase-coherence length).

\section{E. Shot noise}

The shot-noise power $P$, defined in Eq. (34), contains information on temporal correlations in the current that are not contained in the conductance. A familiar example is a tunnel diode, where $P=2 e \bar{I} \equiv P_{\text {Poisson }}$, with
$\bar{I}$ the time-averaged current. This tells us that the electrons traverse the conductor in completely uncorrelated fashion, as in a Poisson process. In a degenerate electron gas the shot noise can be smaller than $P_{\text {Poisson }}$, due to correlations in the electron transmission imposed by the Pauli principle (Kulik and Omel'yanchuk, 1984; Khlus, 1987; Lesovik, 1989; Büttiker, 1990, 1992; Yurke and Kochanski, 1990; Martin and Landauer, 1992). Here we consider the sub-Poissonian shot noise in a metallic diffusive conductor, relevant for random-matrix theory. For reviews specifically devoted to shot noise, see Martin (1994) and De Jong and Beenakker (1997).

Recall is the relationship (35) between the zerotemperature, zero-frequency shot-noise power $P$ and the transmission eigenvalues $T_{n}$. One sees that $P=2 e V G=P_{\text {Poisson }}$ for a conductor, where all $T_{n} \ll 1$ (such as a high tunnel barrier). However, if some $T_{n}$ are near 1 (open channels), then the shot noise is reduced below $P_{\text {Poisson. }}$. In the metallic diffusive regime $(l \ll L \ll N l)$ the variables $x_{n}$ have the uniform density (191). This means that the transmission eigenvalues $T_{n}=1 / \cosh ^{2} x_{n}$ have a bimodal distribution,

$$
\rho_{0}(T)=\frac{N l}{2 L} \frac{1}{T \sqrt{1-T}}, \quad e^{-2 L / l} \leqslant T \leqslant 1,
$$

with a peak at unit transmission and a peak at exponentially small transmission (Dorokhov, 1984; Imry, 1986a; Pendry, MacKinnon, and Roberts, 1992). Averaging Eq. (35) with the density (251) yields

$$
\langle P\rangle=P_{0} \int_{0}^{1} d T T(1-T) \rho_{0}(T)=P_{0} \frac{N l}{3 L}=\frac{1}{3} P_{\text {Poisson }},
$$

where we have used $P_{0} N l / L=2 e V\langle G\rangle=P_{\text {Poisson }}$. The bimodal distribution of the transmission eigenvalues causes a one-third suppression of the shot noise (Beenakker and Büttiker, 1992).

Although the derivation of the eigenvalue density in Sec. III.B.3 is based on the DMPK equation, and hence requires a wire geometry, its validity is independent of the dimensionality of the conductor (Altshuler, Levitov, and Yakovets, 1994; Nazarov, 1994a). ${ }^{23}$ Furthermore, although the concept of a transmission eigenvalue requires phase coherence, this is not required for the onethird suppression. An alternative derivation exists that starts from a semiclassical kinetic equation in which the Pauli principle is accounted for but the electron motion is treated classically (Nagaev, 1992; De Jong and Beenakker, 1995, 1996). The one-third suppression thus applies regardless of whether $L$ is long or short compared to the phase-coherence length $l_{\phi}$.

Loss of phase coherence is one consequence of electron-electron interactions. Another consequence is

\footnotetext{
${ }^{23}$ Corrections to Eq. (252) from the weak-localization effect do depend on the dimensionality. In a wire geometry the weak-localization correction is $\delta P=-\frac{4}{45} P_{0}$ in zero magnetic field (De Jong and Beenakker, 1992).
} 


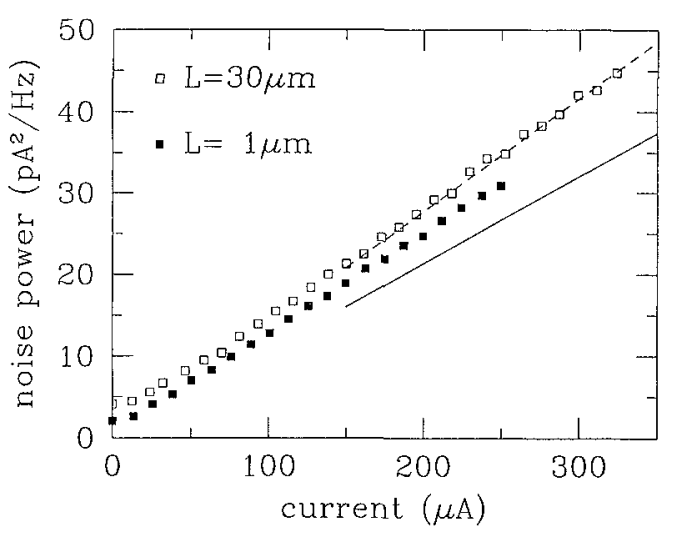

FIG. 26. Current dependence of the noise power at $50 \mathrm{mK}$ in two thin-film $\mathrm{Ag}$ wires of different length $L$. The noise power is linear in the current, indicating shot noise, except at the lowest currents, where thermal noise $P_{\text {thermal }}=4 k_{\mathrm{B}} T G$ takes over. (The conductance $G=0.76 \Omega^{-1}$ for $L=1 \mu \mathrm{m}$ and $1.47 \Omega^{-1}$ for $L=30 \mu \mathrm{m}$.) A current- and temperatureindependent background noise has been subtracted from the data. The lines indicate the two theoretical predictions $P / P_{\text {Poisson }}=\frac{1}{3}$ for short wires (solid) and $P / P_{\text {Poisson }}=\frac{1}{4} \sqrt{3}$ for long wires (dashed). After Steinbach, Martinis, and Devoret (1996).

thermalization of the distribution of the electrons among the available energy levels. The thermalization length $l_{\text {th }}$ is generally much greater than $l_{\phi}$. [Thermalization requires interactions with large transfer of energy, while a small transfer of energy is sufficient to destroy phase coherence (Altshuler and Aronov, 1985; Imry, 1996).] If $L$ becomes greater than $l_{\text {th }}$ the shot-noise power increases slightly, from $P=\frac{1}{3} P_{\text {Poisson }}=0.67 e \bar{I}$ to $P=\frac{1}{4}$ $\sqrt{3} P_{\text {Poisson }}=0.87 e \bar{l}$ (Kozub and Rudin, 1995; Nagaev, 1995; Steinbach, Martinis, and Devoret, 1995; De Jong and Beenakker, 1996). On longer length scales $L>l_{\text {in }}$, inelastic electron-phonon scattering equilibrates the electron gas with the lattice, thereby averaging the shot noise out to zero.

The length-scale dependence of the shot-noise power has been studied experimentally by Steinbach, Martinis, and Devoret (1996) on Ag thin-film wires of different lengths. (Sub-Poissonian shot noise had earlier been measured by Liefrink et al. (1994), on a narrow twodimensional electron gas in a GaAs/AlGaAs heterostructure.) The data for $L=1 \mu \mathrm{m}$ and $30 \mu \mathrm{m}$ at temperature $T=50 \mathrm{mK}$ is shown in Fig. 26. The noise power is linear in the average current for $\bar{I} \geq 50 \mu \mathrm{A}$. (The saturation at smaller currents is due to the residual thermal noise $P_{\text {thermal }}=4 k_{\mathrm{B}} T G$.) The ratio $P / 2 e \bar{I}$ is close to $\frac{1}{4} \sqrt{3}$ (dashed line) for the $30 \mu \mathrm{m}$ wire, indicating that this length is in the range $l_{\mathrm{th}} \ll L \ll l_{\text {in }}$. For the $1 \mu \mathrm{m}$ wire the slope is clearly smaller than in the longer wire but still above the $\frac{1}{3}$ prediction for $L \ll l_{\text {th }}$ - presumably because $L$ is not quite small enough in the experiment.
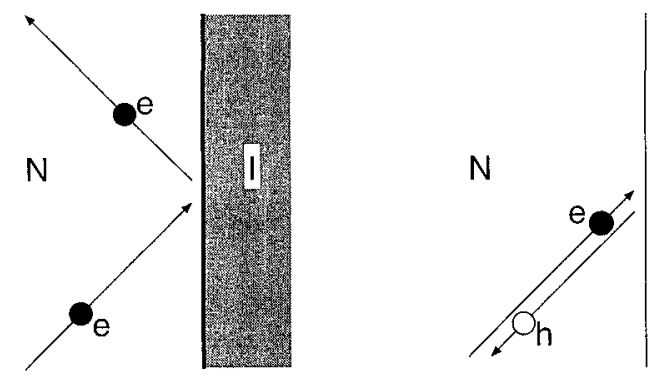

$\mathrm{S}$

FIG. 27. Normal reflection by an insulator (I) versus Andreev reflection by a superconductor $(S)$ of an electron excitation in a normal metal $(\mathrm{N})$ near the Fermi level. Normal reflection (left) conserves charge but does not conserve momentum. Andreev reflection (right) conserves momentum but does not conserve charge: The electron $(e)$ is reflected as a hole (h) with the same momentum and opposite velocity. The missing charge of $2 e$ is absorbed as a Cooper pair by the superconducting condensate.

\section{NORMAL-METAL-SUPERCONDUCTOR JUNCTIONS}

\section{A. Scattering theory}

\section{Andreev reflection}

At the interface between a normal metal and a superconductor, dissipative electrical current is converted into dissipationless supercurrent. The mechanism for this conversion was discovered in 1964 by Andreev-an electron excitation slightly above the Fermi level in the normal metal is reflected at the interface as a hole excitation slightly below the Fermi level (see Fig. 27). The missing charge of $2 e$ is removed as a Cooper pair. The reflected hole has (approximately) the same momentum as the incident electron, but with a velocity in the opposite direction. (The two momenta are precisely equal at the Fermi level.) This curious scattering process is known as retroreflection or Andreev reflection.

The early theoretical work on the conductance of a normal-metal-superconductor (NS) junction treats the dynamics of the quasiparticle excitations semiclassically, as is appropriate for macroscopic junctions. Phase coherence of the electrons and the Andreev-reflected holes is ignored. Interest in "mesoscopic" NS junctions, where phase coherence plays an important role, is a recent development. Significant advances have been made in our understanding of quantum-interference effects due to phase-coherent Andreev reflection. Much of the motivation has come from the technological advances in the fabrication of a highly transparent contact between a superconducting film and the two-dimensional electron gas in a semiconductor heterostructure. The advantages of a two-dimensional electron gas over a metal are the large Fermi wavelength, large mean free path, and the possibility to confine the electrons electrostatically by means of gate electrodes. Andreev reflection requires relatively transparent NS interfaces. Semiconductorsuperconductor junctions are convenient, since the 


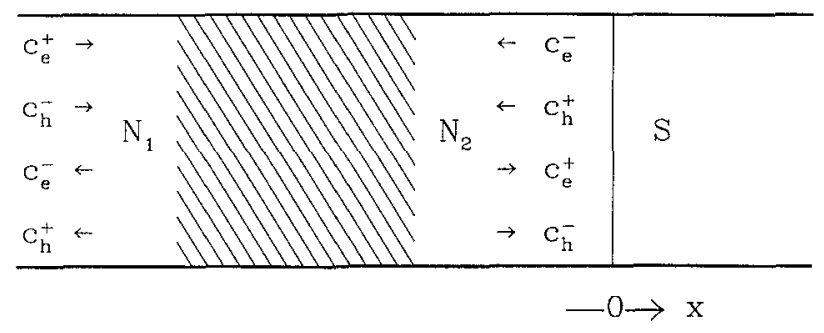

FIG. 28. Normal-metal-superconductor (NS) junction containing a disordered normal region (shaded). Scattering states $c$ in the two normal leads $N_{1}$ and $N_{2}$ are indicated schematically.

Schottky barrier at the interface is much more transparent than a typical dielectric tunnel barrier. The technological effort is directed towards making the interface as transparent as possible.

The random-matrix theory of phase-coherent Andreev reflection is based on a scattering formulation in which the conductance $G_{\mathrm{NS}}$ of the NS junction is related to the transmission matrix $t$ in the normal state. In the limit of zero temperature, zero voltage, and zero magnetic field, the relationship is (Beenakker, 1992a)

$$
G_{\mathrm{NS}}=\frac{4 e^{2}}{h} \sum_{n=1}^{N} \frac{T_{n}^{2}}{\left(2-T_{n}\right)^{2}},
$$

where the transmission eigenvalue $T_{n}$ is an eigenvalue of the matrix product $t t^{\dagger}$. The same numbers $T_{n}$ $(n=1,2, \ldots, N)$ determine the conductance $G_{\mathrm{N}}$ in the normal state, according to the Landauer formula (33). (In this section we append the subscript $\mathrm{N}$ to the normal-state conductance, to help distinguish it from $G_{N S}$.) The fact that the same eigenvalues determine both $G_{N}$ and $G_{\mathrm{NS}}$ means that one can use the same random-matrix ensembles as in the normal state. This is a substantial technical and conceptual simplification.

Let us discuss how Eq. (253) is obtained.

\section{Bogoliubov-De Gennes equation}

The model considered is illustrated in Fig. 28. It consists of a disordered normal region (shaded) adjacent to a superconductor (S). The disordered region may also contain a geometrical constriction or a tunnel barrier. To obtain a well-defined scattering problem we insert ideal (impurity-free) normal leads $\mathrm{N}_{1}$ and $\mathrm{N}_{2}$ to the left and right of the disordered region. The NS interface is located at $x=0$. We assume that the only scattering in the superconductor consists of Andreev reflection at the NS interface, i.e., we consider the case that the disorder is contained entirely within the normal region. The spatial separation of Andreev and normal scattering is the key simplification that allows us to relate the conductance directly to the normal-state scattering matrix. The model is directly applicable to a superconductor in the clean limit (mean free path in $S$ large compared to the superconducting coherence length $\xi$ ), or to a pointcontact junction (formed by a constriction that is narrow compared to $\xi$ ). In both cases the contribution of scattering within the superconductor to the junction resistance can be neglected.

The scattering states at energy $\varepsilon$ are eigenfunctions of an equation called the Bogoliubov-De Gennes equation (De Gennes, 1966) - although historically it made its first appearance in a paper by Andreev (1964). This equation has the form of two Schrödinger equations for electron and hole wave functions $u(\vec{r})$ and $v(\vec{r})$, coupled by the pair potential $\Delta(\vec{r})$ :

$$
\left(\begin{array}{cc}
\mathcal{H}_{0} & \Delta \\
\Delta^{*} & -\mathcal{H}_{0}^{*}
\end{array}\right)\left(\begin{array}{l}
u \\
v
\end{array}\right)=\varepsilon\left(\begin{array}{l}
u \\
v
\end{array}\right) .
$$

Here $\mathcal{H}_{0}$ is the single-electron Hamiltonian, which we assume to be independent of the electron spin. (We will include spin-orbit scattering later.) The excitation energy $\varepsilon$ is measured relative to the Fermi energy $E_{\mathrm{F}}$. To simplify construction of the scattering basis we assume that the magnetic field $\vec{B}$ (in the $z$ direction) vanishes outside the disordered region.

The pair potential in the bulk of the superconductor $(x \geqslant \xi)$ has amplitude $\Delta_{0}$ and phase $\phi$. The spatial dependence of $\Delta(\vec{r})$ near the NS interface is determined by the self consistency relation

$$
\Delta(\vec{r})=g(\vec{r}) \sum_{\varepsilon>0} v^{*}(\vec{r}) u(\vec{r})[1-2 f(\varepsilon)]
$$

where the sum is over all states with positive eigenvalue, ${ }^{24}$ and $f(\varepsilon)=\left[1+\exp \left(\varepsilon / k_{\mathrm{B}} T\right)\right]^{-1}$ is the Fermi function. The coefficient $g$ is the interaction constant of the BCS theory of superconductivity. At an NS interface, $g$ drops abruptly (over atomic distances) to zero, in the assumed absence of any pairing interaction in the normal region. Therefore, $\Delta(\vec{r}) \equiv 0$ for $x<0$. At the superconducting side of the NS interface, $\Delta(\vec{r})$ recovers its bulk value $\Delta_{0} e^{i \phi}$ only at some distance from the interface. We will neglect the suppression of $\Delta(\vec{r})$ on approaching the NS interface and use the step-function model

$$
\Delta(\vec{r})=\Delta_{0} e^{i \phi} \theta(x) .
$$

This model is also referred to in the literature as a "rigid boundary condition." Likharev (1979) discusses in detail the conditions for its validity. If the width $W$ of the NS junction is small compared to $\xi$, the nonuniformities in $\Delta(\vec{r})$ extend only over a distance of order $W$ from the junction (because of "geometrical dilution" of the influence of the narrow junction in the wide superconductor). Since nonuniformities on length scales $\ll \xi$ do not affect the dynamics of the quasiparticles, these can be neglected, and the step-function model holds. A point contact or microbridge belongs in general to this class of junctions. Alternatively, the step-function model also holds for a wide junction if the resistivity of the junction

\footnotetext{
${ }^{24} \mathrm{~A}$ cutoff at $\hbar \omega_{\mathrm{D}}$, with $\omega_{\mathrm{D}}$ the Debye frequency, has to be introduced as usual in the BCS theory.
} 
region is much bigger than the resistivity of the bulk superconductor. A semiconductor-superconductor junction is typically in this second category. Note that both cases are consistent with our assumption that the disorder is contained entirely within the normal region.

It is worth emphasizing that the absence of a pairing interaction in the normal region $[g(\vec{r}) \equiv 0$ for $x<0] \mathrm{im}$ plies a vanishing pair potential $\Delta(\vec{r})$, according to Eq. (255), but does not imply a vanishing order parameter $\Psi(\vec{r})$, which is given by

$$
\Psi(\vec{r})=\sum_{\varepsilon>0} v^{*}(\vec{r}) u(\vec{r})[1-2 f(\varepsilon)] .
$$

Phase coherence between the electron and hole wave functions $u$ and $v$ leads to $\Psi(\vec{r}) \neq 0$ for $x<0$. The term "proximity effect" can therefore mean two different things. One is the suppression of the pair potential $\Delta$ at the superconducting side of the NS interface. This is a small effect that we neglect. The other is the induction of a nonzero order parameter $\Psi$ at the normal side of the NS interface. This effect is fully included, even though $\Psi$ does not appear explicitly in the expressions which follow. The reason is that the order parameter quantifies the degree of phase coherence between electrons and holes but does not itself affect the dynamics of the quasiparticles. [The Bogoliubov-De Gennes equation (254) contains $\Delta$ not $\Psi$.]

\section{Scattering formula for the conductance}

We now construct a basis for the scattering matrix. In the normal lead $\mathrm{N}_{2}$ the eigenfunctions of the Bogoliubov-De Gennes equation (254) can be written in the form

$$
\begin{aligned}
& \psi_{n, \mathrm{e}}^{ \pm}\left(\mathrm{N}_{2}\right)=\left(\begin{array}{l}
1 \\
0
\end{array}\right) \Phi_{n}(y, z) \exp \left( \pm i k_{n}^{\mathrm{e}} x\right), \\
& \psi_{n, \mathrm{~h}}^{ \pm}\left(\mathrm{N}_{2}\right)=\left(\begin{array}{l}
0 \\
1
\end{array}\right) \Phi_{n}(y, z) \exp \left( \pm i k_{n}^{\mathrm{h}} x\right),
\end{aligned}
$$

where the wave numbers $k_{n}^{\mathrm{e}}$ and $k_{n}^{\mathrm{h}}$ are given by

$$
k_{n}^{\mathrm{e}, \mathrm{h}} \equiv\left(2 m / \hbar^{2}\right)^{1 / 2}\left(E_{\mathrm{F}}-E_{n}+\sigma^{\mathrm{e}, \mathrm{h}} \varepsilon\right)^{1 / 2}
$$

and we have defined $\sigma^{\mathrm{e}} \equiv 1, \sigma^{\mathrm{h}} \equiv-1$. The labels e and h indicate the electron or hole character of the wave function. The index $n$ labels the modes, $\Phi_{n}(y, z)$ is the transverse wave function of the $n$th mode and $E_{n}$ its threshold energy. The $\Phi_{n}$ 's are normalized such that each wave function in the basis (258) carries the same amount of quasiparticle current. The eigenfunctions in lead $\mathrm{N}_{1}$ are chosen similarly.

A wave incident on the disordered normal region is described in the basis (258) by a vector of coefficients

$$
c_{\mathrm{N}}^{\mathrm{in}} \equiv\left(c_{\mathrm{e}}^{+}\left(\mathrm{N}_{1}\right), c_{\mathrm{e}}^{-}\left(\mathrm{N}_{2}\right), c_{\mathrm{h}}^{-}\left(\mathrm{N}_{1}\right), c_{\mathrm{h}}^{+}\left(\mathrm{N}_{2}\right)\right)
$$

(The mode index $n$ has been suppressed for simplicity of notation.) The reflected and transmitted wave has vector of coefficients

$$
c_{\mathrm{N}}^{\text {out }} \equiv\left(c_{\mathrm{e}}^{-}\left(\mathrm{N}_{1}\right), c_{\mathrm{e}}^{+}\left(\mathrm{N}_{2}\right), c_{\mathrm{h}}^{+}\left(\mathrm{N}_{1}\right), c_{\mathrm{h}}^{-}\left(\mathrm{N}_{2}\right)\right) .
$$

The scattering matrix $s_{\mathrm{N}}$ of the normal region relates these two vectors,

$$
c_{\mathrm{N}}^{\text {out }}=s_{\mathrm{N}} c_{\mathrm{N}}^{\mathrm{in}} \text {. }
$$

Because the normal region does not couple electrons and holes, this matrix has the block-diagonal form

$$
\begin{aligned}
& s_{\mathrm{N}}(\varepsilon)=\left(\begin{array}{cc}
s_{0}(\varepsilon) & 0 \\
0 & s_{0}(-\varepsilon)^{*}
\end{array}\right), \\
& s_{0} \equiv\left(\begin{array}{ll}
r_{11} & t_{12} \\
t_{21} & r_{22}
\end{array}\right) .
\end{aligned}
$$

Here $s_{0}$ is the unitary scattering matrix associated with the single-electron Hamiltonian $\mathcal{H}_{0}$. The reflection and transmission matrices $r(\varepsilon)$ and $t(\varepsilon)$ are $N \times N$ matrices, $N(\varepsilon)$ being the number of propagating modes at energy $\varepsilon$. (We assume for simplicity that the number of modes in leads $\mathrm{N}_{1}$ and $\mathrm{N}_{2}$ is the same.) The matrix $s_{0}$ is unitary $\left(s_{0} s_{0}^{\dagger}=1\right)$ and satisfies the symmetry relation $s_{0}(\varepsilon, B)_{l j}=s_{0}(\varepsilon,-B)_{l}$.

For energies $0<\varepsilon<\Delta_{0}$ there are no propagating modes in the superconductor. We can then define a scattering matrix for Andreev reflection at the NS interface that relates the vector of coefficients $\left(c_{\mathrm{e}}^{-}\left(\mathrm{N}_{2}\right), c_{\mathrm{h}}^{+}\left(\mathrm{N}_{2}\right)\right)$ to $\left(c_{\mathrm{e}}^{+}\left(\mathrm{N}_{2}\right), c_{\mathrm{h}}^{-}\left(\mathrm{N}_{2}\right)\right)$. The elements of this scattering matrix can be obtained by matching the wave functions (258) at $x=0$ to the decaying solutions in $S$ of the Bogoliubov-De Gennes equation. If terms of order $\Delta_{0} / E_{\mathrm{F}}$ are neglected (the so-called Andreev approximation), the result is simply

$$
\begin{aligned}
& c_{\mathrm{e}}^{-}\left(\mathrm{N}_{2}\right)=\alpha e^{l \phi} c_{\mathrm{h}}^{-}\left(\mathrm{N}_{2}\right), \\
& c_{\mathrm{h}}^{+}\left(\mathrm{N}_{2}\right)=\alpha e^{-\imath \phi} c_{\mathrm{e}}^{+}\left(\mathrm{N}_{2}\right),
\end{aligned}
$$

where $\alpha \equiv \exp \left[-i \arccos \left(\varepsilon / \Delta_{0}\right)\right]$. Andreev reflection transforms an electron mode into a hole mode, without change of mode index. The transformation is accompanied by a phase shift, which consists of two parts: (1) a phase shift $-\arccos \left(\varepsilon / \Delta_{0}\right)$ due to the penetration of the wave function into the superconductor; (2) a phase shift equal to plus or minus the phase $\phi$ of the pair potential in the superconductor (plus for reflection from hole to electron, minus for the reverse process).

We can combine the $2 N$ linear relations of Eq. (264) with the $4 N$ relations of Eq. (262) to obtain a set of $2 N$ linear relations between the incident wave in lead $\mathrm{N}_{1}$ and the reflected wave in the same lead:

$$
\begin{aligned}
& c_{\mathrm{e}}^{-}\left(\mathrm{N}_{1}\right)=s_{\mathrm{ee}} c_{\mathrm{e}}^{+}\left(\mathrm{N}_{1}\right)+s_{\mathrm{eh}} c_{\mathrm{h}}^{-}\left(\mathrm{N}_{1}\right), \\
& c_{\mathrm{h}}^{+}\left(\mathrm{N}_{1}\right)=s_{\mathrm{he}} c_{\mathrm{e}}^{+}\left(\mathrm{N}_{1}\right)+s_{\mathrm{hh}} c_{\mathrm{h}}^{-}\left(\mathrm{N}_{1}\right) .
\end{aligned}
$$

The four $N \times N$ matrices $s_{\mathrm{ee}}, s_{\mathrm{hh}}, s_{\mathrm{eh}}$, and $s_{\mathrm{he}}$ together form the scattering matrix $s$ of the whole system for energies $0<\varepsilon<\Delta_{0}$. An electron incident in lead $\mathrm{N}_{1}$ is reflected either as an electron (with scattering amplitudes $s_{\text {ee }}$ ) or as a hole (with scattering amplitudes $s_{\text {he }}$ ). Similarly, the matrices $s_{\mathrm{hh}}$ and $s_{\mathrm{eh}}$ contain the scattering 
amplitudes for reflection of a hole as a hole or as an electron. After some algebra we find for these matrices the expressions

$$
\begin{aligned}
& s_{\mathrm{ee}}(\varepsilon)=r_{11}(\varepsilon)+\alpha^{2} t_{12}(\varepsilon) r_{22}^{*}(-\varepsilon) M_{\mathrm{e}} t_{21}(\varepsilon) \\
& s_{\mathrm{hh}}(\varepsilon)=r_{11}^{*}(-\varepsilon)+\alpha^{2} t_{12}^{*}(-\varepsilon) r_{22}(\varepsilon) M_{\mathrm{h}} t_{21}^{*}(-\varepsilon) \\
& s_{\mathrm{eh}}(\varepsilon)=\alpha e^{\imath \phi} t_{12}(\varepsilon) M_{\mathrm{h}} t_{21}^{*}(-\varepsilon) \\
& s_{\mathrm{he}}(\varepsilon)=\alpha e^{-\imath \phi} t_{12}^{*}(-\varepsilon) M_{e} t_{21}(\varepsilon)
\end{aligned}
$$

where we have defined the matrices

$$
\begin{aligned}
& M_{\mathrm{e}} \equiv\left[1-\alpha^{2} r_{22}(\varepsilon) r_{22}^{*}(-\varepsilon)\right]^{-1}, \\
& M_{\mathrm{h}} \equiv\left[1-\alpha^{2} r_{22}^{*}(-\varepsilon) r_{22}(\varepsilon)\right]^{-1} .
\end{aligned}
$$

One can verify that the scattering matrix constructed from these four submatrices satisfies unitarity $\left(s s^{\dagger}\right.$ $=1)$ and the symmetry relation $s(\varepsilon, B, \phi)_{l}$ $=s(\varepsilon,-B,-\phi)_{l l}$, as required by quasiparticle-current conservation and by time-reversal invariance, respectively.

The differential conductance $G_{\mathrm{NS}}$ of the NS junction at zero temperature and subgap voltage $V \leqslant \Delta_{0} / e$ is given by (Blonder, Tinkham, and Klapwijk, 1982; Lambert, 1991; Takane and Ebisawa, 1992a)

$$
\begin{aligned}
G_{\mathrm{NS}} & =G_{0} \operatorname{Tr}\left(1-s_{\mathrm{ee}} s_{\mathrm{ee}}^{\dagger}+s_{\mathrm{he}} s_{\mathrm{he}}^{\dagger}\right) \\
& =2 G_{0} \operatorname{Tr} s_{\mathrm{he}} s_{\mathrm{he}}^{\dagger}=2 G_{0} \operatorname{Tr} s_{\mathrm{eh}} s_{\mathrm{el}}^{\dagger} .
\end{aligned}
$$

(The second and third equalities follow from unitarity of s.) The conductance quantum $G_{0}=2 e^{2} / h$, the factor of two being due to spin degeneracy. The scattering matrix elements are to be evaluated at energy $\varepsilon=e V$. We now substitute Eq. (266c) into Eq. (268) and obtain the expression (Beenakker, 1992a)

$$
\begin{aligned}
& G_{\mathrm{NS}}=2 G_{0} \operatorname{Tr} m(e V) m^{\dagger}(e V), \\
& m(\varepsilon)=t_{12}(\varepsilon)\left[1-\alpha^{2} r_{22}^{*}(-\varepsilon) r_{22}(\varepsilon)\right]^{-1} t_{21}^{*}(-\varepsilon) .
\end{aligned}
$$

The advantage of Eq. (269) over Eq. (268) is that it can be evaluated with the same techniques developed for quantum transport in the normal-state, since the only input is the normal-state scattering matrix. The effects of multiple Andreev reflections are fully incorporated by the matrix inversion in Eq. (269b).

In the limit $V \rightarrow 0$ of linear response we only need the scattering matrix elements at the Fermi level, i.e., at $\varepsilon=0$. We will restrict ourselves to this limit in most of what follows and omit the argument $\varepsilon$. Note that $\alpha=-i$ for $\varepsilon=0$. In the absence of a magnetic field, the Eq. (269) simplifies considerably. Since the scattering matrix $s_{0}$ of the normal region is symmetric for $B=0$, one has $r_{22}^{*}=r_{22}^{1}$ and $t_{21}^{*}=t_{12}^{\dagger}$. Equation (269) then takes the form

$$
\begin{aligned}
G_{\mathrm{NS}} & =2 G_{0} \operatorname{Tr} t_{12}\left(1+r_{22}^{\dagger} r_{22}\right)^{-1} t_{12}^{\dagger} t_{12}\left(1+r_{22}^{\dagger} r_{22}\right)^{-1} t_{12}^{\dagger} \\
& =2 G_{0} \operatorname{Tr}\left(t_{12}^{\dagger} t_{12}\left(2-t_{12}^{\dagger} t_{12}\right)^{-1}\right)^{2} .
\end{aligned}
$$

In the second equality we have used the unitarity relation $r_{22}^{\dagger} r_{22}+t_{12}^{\dagger} t_{12}=1$. The trace (270) depends only on the eigenvalues $T_{n}(n=1,2, \ldots, N)$ of the transmission matrix product $t_{12}^{\dagger} t_{12}$. We thus obtain the relation between the conductance and the transmission eigenvalues given in Eq. (253). This relation holds for an arbitrary transmission matrix $t$, i.e., for arbitrary disorder potential. It is the multichannel generalization of a formula first obtained by Blonder, Tinkham, and Klapwijk (1982) (and subsequently by Shelankov, 1984, and Zar̆tsev, 1984) for the single-channel case (appropriate for a geometry such as a planar tunnel barrier, where the different scattering channels are uncoupled).

Slevin, Pichard, and Mello (1996; Altland and Zirnbauer, 1996b; Brouwer and Beenakker, 1996a) have considered the modifications required by the inclusion of spin-orbit scattering. The scattering matrix elements are then quaternion numbers. The complex conjugate $Q^{*}$ and the Hermitian conjugate $Q^{i}$ of a matrix $Q$ with quaternion elements $Q_{n m}=a_{n m} \mathbb{1}+i b_{n m} \sigma_{x}+i c_{n m} \sigma_{y}$ $+i d_{n m} \sigma_{z}$ have matrix elements

$$
\begin{aligned}
& Q_{n m}^{*}=a_{n m}^{*} 1+i b_{n m}^{*} \sigma_{x}+i c_{n m}^{*} \sigma_{y}+i d_{n m}^{*} \sigma_{z}, \\
& Q_{n m}^{\prime}=a_{m n}^{*} 1-i b_{m n}^{*} \sigma_{x}-i c_{m n}^{*} \sigma_{y}-i d_{m n}^{*} \sigma_{z} .
\end{aligned}
$$

Notice that the definition of the Hermitian conjugate of the $N \times N$ quaternion matrix $Q$ is the same as for the corresponding $2 N \times 2 N$ complex matrix, while the definition of the complex conjugate is different. The dual $Q^{\mathrm{R}}$ of a quaternion matrix is defined by $Q^{\mathrm{R}}=\left(Q^{*}\right)^{\dagger}$, which differs from the transpose of a complex matrix. The trace of $Q$ is defined by $\operatorname{Tr} Q=\Sigma_{n} a_{n n}$, which is half the trace of the corresponding $2 N \times 2 N$ matrix. With these definitions Eq. (269) remains valid in the presence of spin-orbit scattering. What about Eq. (253)? In zero magnetic field, $s_{0}$ is a self-dual matrix: $s_{0}=s_{0}^{\mathrm{R}}$. Hence $r_{22}^{*}=r_{22}^{\dagger}$ and $t_{21}^{*}=t_{12}^{\dagger}$, which, combined with unitarity, implies Eq. (270). The linear-response conductance in zero magnetic field is therefore still given by Eq. (253).

In summary, the linear-response conductance of an NS junction is a linear statistic on the transmission eigenvalues for $\beta=1$ or 4 , given by Eq. (253). For $\beta=2$, or for finite voltage, the more general Eq. (269) is required, which is not a linear statistic.

\section{B. Ideal normal-metal-superconductor interface}

In this subsection we investigate the case of an ideal (i.e., perfectly transparent) interface between the normal metal and the superconductor. The effect of a tunnel barrier at the NS interface will be considered in the next subsection. The disordered normal region is supposed to have a length $L$ much greater than its width $W$ (see Fig. 6). We concentrate on the metallic diffusive regime, in which $L$ is greater than the mean free path $l$ for elastic impurity scattering but smaller than the localization length $N l$. 


\section{Average conductance}

Let us begin by calculating the average conductance of the junction, averaged over an ensemble of impurity configurations (Beenakker, 1992a). According to Eq. (253), this average is given in zero magnetic field by

$$
\begin{aligned}
\left\langle G_{\mathrm{NS}}\right\rangle & =\frac{4 e^{2}}{h} \int_{0}^{1} d T \rho(T) \frac{T^{2}}{(2-T)^{2}} \\
& =\frac{4 e^{2}}{h} \int_{0}^{\infty} d x \rho(x) \frac{1}{\cosh ^{2} 2 x},
\end{aligned}
$$

where in the second equality we have substituted the parametrization $T=1 / \cosh ^{2} x$ introduced in Sec. I.C.1. Equation (273) is to be compared with the equation for the average conductance in the normal state,

$$
\left\langle G_{\mathrm{N}}\right\rangle=\frac{2 e^{2}}{h} \int_{0}^{\infty} d x \rho(x) \frac{1}{\cosh ^{2} x},
$$

which follows from the Landauer formula (33). As we discussed in Sec. III.B.3, the density $\rho(x)=N l / L$ $+\mathcal{O}\left(N^{0}\right)$ is uniform in the metallic diffusive regime, up to weak-localization corrections. Ignoring these corrections for the moment, we find that

$$
\left\langle G_{\mathrm{NS}}\right\rangle=\frac{2 e^{2}}{h} \frac{N l}{L}+\mathcal{O}\left(N^{0}\right)=\left\langle G_{\mathrm{N}}\right\rangle .
$$

We conclude that, although $G_{\mathrm{NS}}$ according to Eq. (253) is of second order in the transmission eigenvalues $T_{n}$, the ensemble average $\left\langle G_{\mathrm{NS}}\right\rangle$ is of first order in $l / L$. The resolution of this paradox is that the $T_{n}$ 's are not distributed uniformly but are either exponentially small or of order unity (see Sec. III.E). Hence the average of $T_{n}^{2}$ is of the same order as the average of $T_{n}$.

Differences between $\left\langle G_{\mathrm{NS}}\right\rangle$ and $\left\langle G_{\mathrm{N}}\right\rangle$ may appear because of several effects. One effect is that of a finite temperature. Equation (273) holds if the thermal energy $k_{\mathrm{B}} T$ is much smaller than the Thouless energy $E_{\mathrm{c}}=\hbar D / L^{2}$ (with $D$ the diffusion coefficient). Nazarov and Stoof (1996; see also Golubov, Wilhelm, and Zaikin, 1997; Stoof and Nazarov, 1996a) have calculated that $\left\langle G_{\mathrm{NS}}\right\rangle$ increases by $10 \%$ as $k_{\mathrm{B}} T$ is raised to $E_{\mathrm{c}}$ and then drops back to $\left\langle G_{\mathrm{N}}\right\rangle$ at higher temperatures. Such a nonmonotonic temperature dependence was first predicted for a point-contact geometry by Artemenko, Volkov, and Zaĭtsev (1979). Experimental confirmation has been provided by Charlat et al. (1996). Nazarov and Stoof have also shown that at zero temperature, attractive (repulsive) interactions between the quasiparticles in the normal metal lead to an increase (decrease) of $\left\langle G_{\mathrm{NS}}\right\rangle$ relative to $\left\langle G_{\mathrm{N}}\right\rangle$.

Contact resistances are yet another effect. As discussed in Sec. III.B.1, the contact resistance in the normal state is $h /\left(2 N e^{2}\right)$, so that

$$
\frac{2 N e^{2}}{h}\left\langle G_{\mathrm{N}}\right\rangle^{-1}=\frac{L}{l}+1 .
$$

In an NS junction, the contact resistance is voltage and magnetic-field dependent (Brouwer and Beenakker, 1995b):

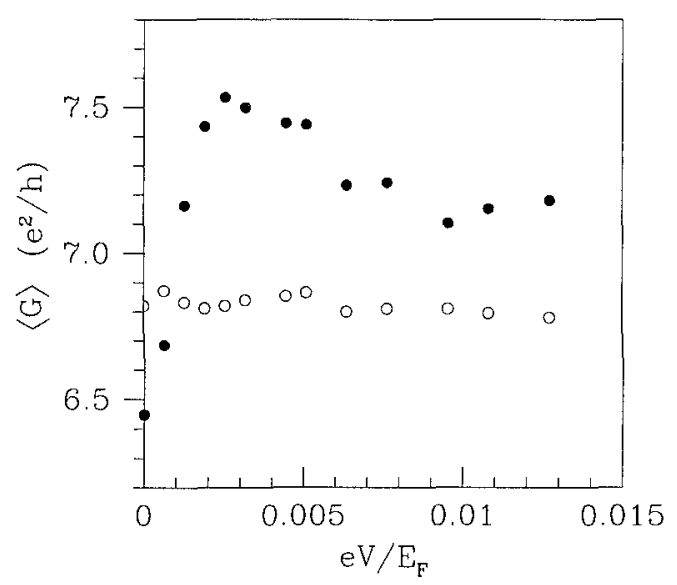

FIG. 29. Numerical simulation of the voltage dependence of the average differential conductance of a two-dimensional wire $(L / W=4.8, N=15, l / L=0.31)$, in zero magnetic field. The filled circles represent $\left\langle G_{\mathrm{NS}}\right\rangle$, for the case that the wire is connected to a superconducting reservoir; the open circles represent the $V$-independent conductance $\left\langle G_{\mathrm{N}}\right\rangle$ in the normal state. At zero voltage, $\left\langle G_{\mathrm{NS}}\right\rangle$ is smaller than $\left\langle G_{\mathrm{N}}\right\rangle$ because of the weak-localization effect. At high voltage, $\left\langle G_{\mathrm{NS}}\right\rangle$ is larger than $\left\langle G_{\mathrm{N}}\right\rangle$ because of the contact-resistance effect. After Brouwer and Beenakker (1995b).

$$
\frac{2 N e^{2}}{h}\left\langle G_{\mathrm{NS}}\right\rangle^{-1}=\left\{\begin{array}{l}
\frac{L}{l}+1 \text { if } B=0 \text { and } V=0, \\
\frac{L}{l}+\frac{1}{2} \text { if } B \gg B_{\mathrm{c}} \text { or } V \gg E_{\mathrm{c}} / e,
\end{array}\right.
$$

where $B_{\mathrm{c}}=h /(e L W)$. At zero voltage and zero magnetic field the contact resistance of the NS junction is the same as in the normal state. Application of either a voltage or a magnetic field reduces the contact resistance in the NS junction by a factor of two. This leads to an increase of $\left\langle G_{\mathrm{NS}}\right\rangle$ by approximately $(l / L)^{2} N e^{2} / h$. A numerical simulation of this contact-resistance effect is shown in Fig. 29, where the differential conductance at zero magnetic field is plotted as a function of voltage. For $V \gg E_{\mathrm{c}} / e,\left\langle G_{\mathrm{NS}}\right\rangle$ is larger than $\left\langle G_{\mathrm{N}}\right\rangle$ because of the difference in contact resistance. The nonmonotonic $V$ dependence at intermediate voltages, observed in the simulation, has been studied theoretically by Yip (1995), Volkov, Allsopp, and Lambert (1996), and Lesovik, Fauchère, and Blatter (1997), and experimentally by Charlat et al. (1996) and Poirier, Mailly, and Sanquer (1996). (It is closely related to the nonmonotonic temperature dependence mentioned above.) The difference between $\left\langle G_{\mathrm{NS}}\right\rangle$ and $\left\langle G_{\mathrm{N}}\right\rangle$ at $V=0$ is due to the term of order $N^{0}$ in Eq. (275). This represents the weaklocalization effect, which we discuss in the following subsection.

\section{Weak localization}

In the presence of time-reversal symmetry, i.e., for $\beta=1$ or 4 , the weak-localization correction to the average conductance of the NS junction can be computed 
TABLE III. Dependence of the weak-localization correction $\delta G_{\mathrm{NS}}$ of a normal-metal wire attached to a superconductor on the presence or absence of time-reversal symmetry (TRS) and electron-hole degeneracy (ehD). The results are for a metal without spin-orbit scattering (Brouwer and Beenakker, 1995b). In the presence of strong spin-orbit scattering each entry is to be multiplied by $-1 / 2$ (Slevin, Pichard, and Mello, 1996). For comparison, the corresponding result in the normal state is listed in braces.

\begin{tabular}{lcc}
\hline \hline$\delta G_{\mathrm{NS}}\left[e^{2} / h\right]$ & TRS & no TRS \\
\hline ehD & $-2+8 / \pi^{2}\{-2 / 3\}$ & $-2 / 3\{0\}$ \\
no ehD & $-4 / 3\{-2 / 3\}$ & $0\{0\}$ \\
\hline \hline
\end{tabular}

from the $\mathcal{O}\left(N^{0}\right)$ correction to the transmissioneigenvalue density. We write $\left\langle G_{\mathrm{NS}}\right\rangle=N l / L+\delta G_{\mathrm{NS}}$ and $\rho(x)=N l / L+\delta \rho(x)$. For $\beta=1$ or 4 , the $\mathcal{O}\left(N^{0}\right)$ corrections $\delta G_{\mathrm{NS}}$ and $\delta \rho(x)$ are related by

$$
\delta G_{\mathrm{NS}}=\frac{4 e^{2}}{h} \int_{0}^{\infty} d x \delta \rho(x) \frac{1}{\cosh ^{2} 2 x},
$$

in view of Eq. (253) and the definition $T=1 / \cosh ^{2} x$. The function $\delta \rho(x)$ is given by Eq. (194). Substitution into Eq. (278) yields the result (Beenakker, 1994b; see also Macêdo and Chalker, 1994; Takane and Otani, 1994; Nazarov, 1995b)

$$
\delta G_{\mathrm{NS}}=(1-2 / \beta)\left(2-\frac{8}{\pi^{2}}\right) \frac{e^{2}}{h}, \quad \text { if } \quad \beta=1,4 .
$$

Equation (279) does not apply to $\beta=2$, because then Eq. (253) for $G_{\text {NS }}$ on which it is based does not hold. Instead, one should start from the more general expression, Eq. (269). It turns out (Brouwer and Beenakker, $1995 \mathrm{~b}$ ) that breaking time-reversal symmetry is not sufficient to suppress the weak-localization correction in an NS junction but only reduces $\delta G_{\mathrm{NS}}$ by about a factor of two (see Table III). To achieve $\delta G_{\mathrm{NS}}=0$ requires, in addition to a magnetic field, a sufficiently large voltage to break the degeneracy in energy between the electrons (occupied states at energy $e V$ above the Fermi level) and the holes (empty states at energy $e V$ below the Fermi level). The electron-hole degeneracy is effectively broken when $e V$ exceeds the Thouless energy $E_{\mathrm{c}}$. Weak localization in an NS junction coexists with a magnetic field, as long as $e V \ll E_{c}$.

All this is in marked contrast with weak localization in the normal state, where $\delta G$ vanishes in a magnetic field regardless of the voltage. (In fact, $\delta G$ is independent of $V$ on the scale of $E_{\mathrm{c}}$.) In normal metals, weak localization is understood (Bergmann, 1984; Khmel'nitskir̆, 1984) as constructive interference of pairs of timereversed Feynman paths [Fig. 30(a)]. This interference is destroyed by a magnetic field. What kind of interfering paths are responsible for weak localization in an NS junction without time-reversal symmetry? The two simplest interfering paths are shown in Fig. 30(b). Regardless of whether time-reversal symmetry is broken or not, there is an exact cancellation of the phase shifts accumulated by the electron and the hole that traverse the loop

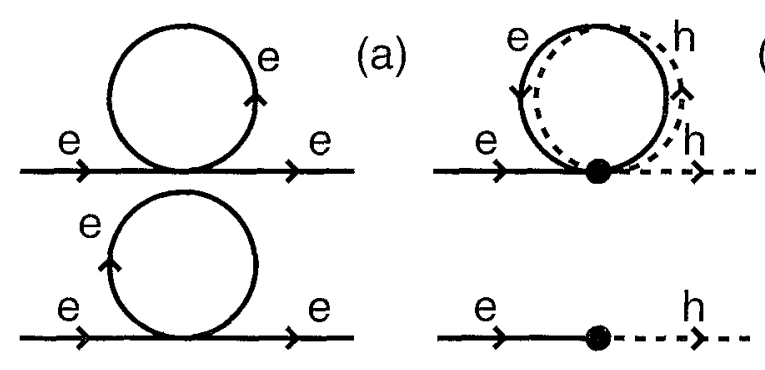

(b)

FIG. 30. Interfering Feynman paths of electrons $e$ and holes $h$. (a) Two paths interfering constructively in the presence of time-reversal symmetry. (b) Two paths involving Andreev reflection (solid dot), which interfere destructively both in the presence and absence of time-reversal symmetry. In the normal state, weak localization (meaning a negative correction to the conductance of order $e^{2} / h$ ) arises from the paths in (a). In a normal-metal-superconducting junction, weak localization coexists in the presence of a magnetic field because of the paths in (b). After Brouwer and Beenakker (1995b).

in the same direction. What remains is a phase shift of $\pi$ due to the double Andreev reflection. As a consequence, the path with the double loop interferes destructively with the path without a loop, giving rise to a negative $\delta G_{\mathrm{NS}}$. In the diagrammatic perturbation theory of weak localization (Anderson, Abrahams, and Ramakrishnan, 1979; Gor'kov, Larkin, and Khmel'nitskiř, 1979), the two interfering time-reversed paths of Fig. 30(a) correspond to a diagram known as the cooperon. The two paths involving Andreev reflection of Fig. 30(b) correspond to a new type of diagram, first identified by Altland and Zirnbauer (1996).

The interested reader is referred to Appendix $\mathrm{D}$ for the calculation of $\delta G_{\mathrm{NS}}$. The results, summarized in Table III, imply a universal $B$ and $V$ dependence of the conductance of a NS microbridge. Raising first $B$ and then $V$ leads to two subsequent increases of the conductance, while raising first $V$ and then $B$ leads first to a decrease and then to an increase. The $V$ dependence of the differential conductance in a time-reversal symmetry-breaking magnetic field is shown in Fig. 31. The dots are numerical simulations of the Anderson model, while the arrows indicate the increase of $\left\langle G_{\mathrm{NS}}\right\rangle$ by $\frac{2}{3} e^{2} / h$ predicted by Table III. The agreement is quite satisfactory. The $V$ dependence of the weak-localization correction at zero $B$ (or the $B$ dependence at zero $V$ ) is obscured by the $B$ - and $V$-dependent contact resistance of the previous subsection, which can only be neglected if $N(l / L)^{2} \ll 1$. This condition is difficult to meet in numerical simulations and possibly also in experiments. This complication was not understood in earlier simulations by Marmorkos, Beenakker, and Jalabert (1993) and experiments by Lenssen et al. (1994).

\section{Universal conductance fluctuations}

So far we have considered the ensemble average $\left\langle G_{\mathrm{NS}}\right\rangle$ of the conductance of the NS junction. In Fig. 32 we show results of numerical simulations by Marmor- 


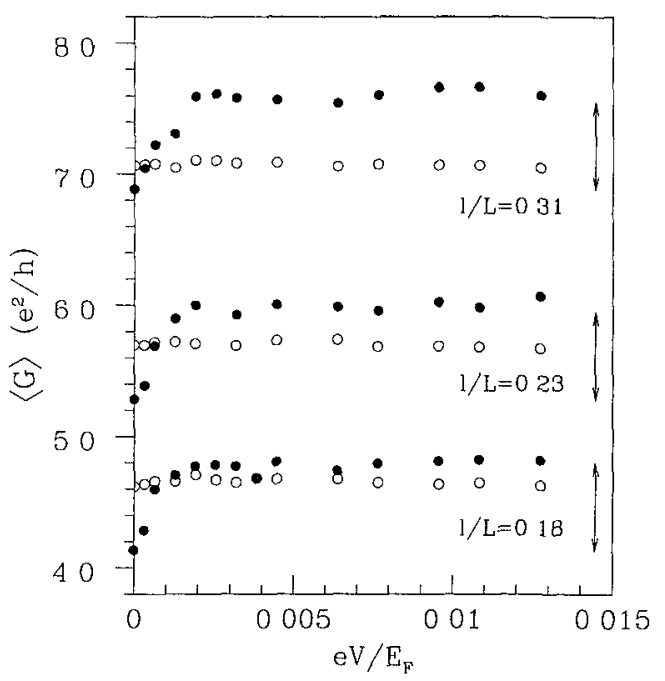

FIG. 31. Numerical simulation of the voltage dependence of the average differential conductance of a two-dimensional wire $(L / W=4.8, N=15)$, for a magnetic flux of $6 h / e$ through the disordered region. The filled circles represent $\left\langle G_{\mathrm{NS}}\right\rangle$, the open circles $\left\langle G_{N}\right\rangle$. The three sets of data points are for three different values of the ratio $l / L$. The arrows indicate the theoretically predicted increase of $G_{\mathrm{NS}}$ by $\frac{2}{3} e^{2} / h$, due to the weaklocalization effect. The contact-resistance effect is suppressed by the magnetic field. After Brouwer and Beenakker (1995b).

kos, Beenakker, and Jalabert (1993) for the sample-tosample fluctuations. A range of parameters $L, W, l$, and $N$ was used to collect this data, in the quasi-onedimensional, metallic, diffusive regime $l<W<L<N l$. The normal-state results are in accord with the prediction (51) of the Altshuler-Lee-Stone theory of "universal conductance fluctuations." As implied by the $1 / \beta$ dependence of $\operatorname{Var} G_{\mathrm{N}}$, the variance is reduced by a factor

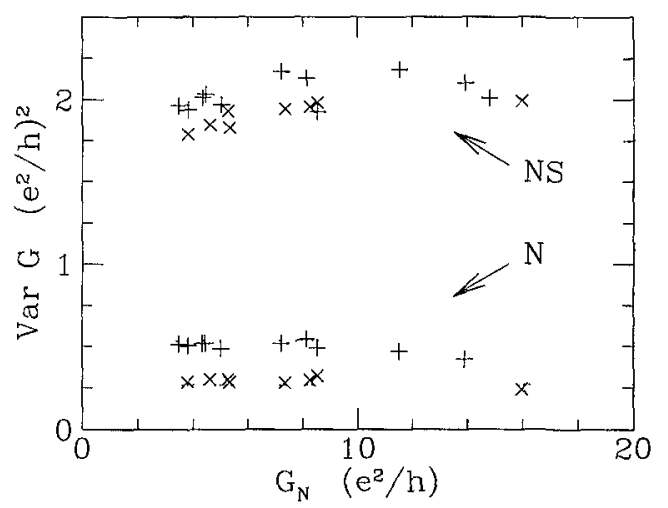

FIG. 32. Numerical simulation of the variance of the conductance of a two-dimensional wire, for different values of the average conductance ( + for $B=0 ; \times$ for a flux of $10 \mathrm{~h} / \mathrm{e}$ through the disordered region). The labels $N$ and NS indicate the case that the wire connects two normal reservoirs or one normal and one superconducting reservoir, respectively. Dotted lines are the analytical results from Eqs. (51) and (280). Note the absence of a factor-of-two reduction in $\operatorname{Var} G_{N S}$ on applying a magnetic field. After Marmorkos, Beenakker, and Jalabert (1993). of two upon application of a time-reversal symmetrybreaking magnetic field (see the two dotted lines in the lower part of Fig. 32). The data for $\operatorname{Var} G_{\mathrm{NS}}$ at $B=0$ shows approximately a fourfold increase over the normal state. For $B \neq 0$, the simulation shows that $\operatorname{Var} G_{\mathrm{NS}}$ is insensitive to a magnetic field. In contrast to the situation in the normal state, the theory for universal conductance fluctuations in an NS junction is quite different for zero and for nonzero magnetic field, as we now discuss.

In zero magnetic field, the conductance of the NS junction is a linear statistic on the transmission eigenvalues, according to Eq. (253). The variance then follows immediately from Eq. (185) for the variance of an arbitrary linear statistic in a wire geometry (Beenakker and Rejaei, 1993; Chalker and Macêdo, 1993). Substitution of $a(x)=\left(4 e^{2} / h\right) \cosh ^{-2} 2 x$ into Eq. (185) yields

$$
\begin{aligned}
\operatorname{Var} G_{\mathrm{NS}} & =\frac{64}{15}\left(1-\frac{45}{\pi^{4}}\right) \beta^{-1}\left(\frac{e^{2}}{h}\right)^{2} \\
& =4.30 \beta^{-1} \operatorname{Var} G_{\mathrm{N}}, \text { if } \beta=1,4 .
\end{aligned}
$$

A factor of four between $\operatorname{Var} G_{\mathrm{NS}}$ and $\operatorname{Var} G_{\mathrm{N}}$ was estimated by Takane and Ebisawa (1992b). [A diagrammatic calculation by the same authors-(Takane and Ebisawa, 1991) - gave a factor of six, presumably because only the dominant diagram was included.] The numerical data in Fig. 32 is within $10 \%$ of the theoretical prediction (280) for $\beta=1$ (upper dotted line). Similar numerical results for $\operatorname{Var} G_{\mathrm{NS}}$ in zero magnetic field were obtained by Takane and Ebisawa (1992b) and Bruun, Hui, and Lambert (1994).

We conclude that the phenomenon of universal conductance fluctuations in zero magnetic field is basically the same for $G_{\mathrm{N}}$ and $G_{\mathrm{NS}}$, because both quantities are linear statistics for $\beta=1,4$. If time-reversal symmetry is broken by a magnetic field, the situation is qualitatively different. For $G_{\mathrm{N}}$, breaking time-reversal symmetry does not affect the universality of the fluctuations but merely reduces the variance by a factor of two. No such simple behavior is to be expected for $G_{\mathrm{NS}}$, since it is no longer a linear statistic for $\beta=2$. Indeed, the numerical data of Fig. 32 demonstrate that $\operatorname{Var} G_{\mathrm{NS}}$ is unaffected by a magnetic field, within the $10 \%$ statistical uncertainty of the simulations. Is there some symmetry principle hidden behind these findings?

Motivated by this question, a calculation of conductance fluctuations in an NS junction for $\beta=2$ was carried out by Brouwer and Beenakker (1995c). The result is that $\operatorname{Var} G_{\mathrm{NS}}$ for a disordered wire attached to a superconductor is reduced by $\left(2-90 / \pi^{4}\right)^{-1}=0.929$ upon breaking time-reversal symmetry (see Table IV). This number is sufficiently close to 1 to be consistent with the numerical simulations but not precisely equal to 1 , so that we can be sure that no rigorous symmetry principle exists. Still, an approximate symmetry argument could be found, as we now discuss. For simplicity, we first assume zero voltage and no spin-orbit scattering.

The argument is based on Eq. (269) for the conductance $G_{\mathrm{NS}}$ of an NS junction, in terms of the scattering 
TABLE IV. Dependence of the variance of the conductance Var $G_{\mathrm{NS}}$ of a normal-metal wire attached to a superconductor on the presence or absence of time-reversal symmetry (TRS) and electron-hole degeneracy (ehD). The results are for a metal without spin-orbit scattering (Brouwer and Beenakker, 1995b). In the presence of strong spin-orbit scattering each entry is to be multiplied by $1 / 4$ (Brouwer and Beenakker, 1996a). For comparison, the corresponding result in the normal state is listed in braces.

\begin{tabular}{lcc}
\hline \hline Var $G_{\text {NS }}\left[e^{4} / h^{2}\right]$ & TRS & no TRS \\
\hline ehD & $64 / 15-192 / \pi^{4}\{8 / 15\}$ & $32 / 15\{4 / 15\}$ \\
no ehD & $32 / 15\{8 / 15\}$ & $16 / 15\{4 / 15\}$ \\
\hline
\end{tabular}

matrix $s_{0}$ of the normal region. We compare $G_{\mathrm{NS}}$ with the conductance $G_{\mathrm{NN}}$ of an entirely normal metal consisting of two segments in series (see Fig. 33). The first segment has scattering matrix $s_{0}$, the second segment is the mirror image of the first. That is to say, the disorder potential is specularly reflected, and the sign of the magnetic field is reversed. The system NN thus has a reflection symmetry (RS), both in the presence and absence of time-reversal symmetry (TRS). The scattering matrix of the second segment is $X s_{0} X$, where $X$ is a $2 N \times 2 N$ matrix with all elements equal to zero, except for $X_{i, N+i}=X_{N+i, i}=1 \quad(i=1,2, \ldots, N)$. (The matrix $X$ exchanges scattering states incident from left and right.) The conductance $G_{\mathrm{NN}}$ follows from the transmission matrix through the two segments in series by means of the Landauer formula,

$$
G_{\mathrm{NN}}(\mathrm{RS})=G_{0} \operatorname{Tr} m^{\prime} m^{\prime \dagger}, \quad m^{\prime}=t_{12}\left[1-\left(r_{22}\right)^{2}\right]^{-1} t_{21} .
$$

The difference between $r^{*} r$ in Eq. (269) and $r^{2}$ in Eq. (281) is crucial in the presence of time-reversal symmetry, but not in its absence. Indeed, an explicit calculation shows that, for broken time-reversal symmetry, the variance of $\operatorname{Tr} m m^{\dagger}$ equals that of $\operatorname{Tr} m^{\prime} m^{\prime \dagger}$, hence

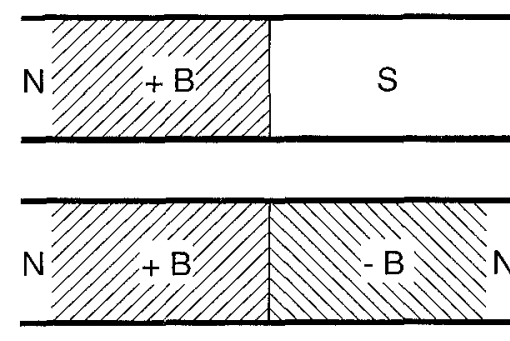

FIG. 33. Exchange of symmetries. (a) Schematic drawing of a disordered normal metal $(\mathrm{N})$ connected to a superconductor (S), in a time-reversal symmetry-breaking magnetic field $B$. In (b) the normal region is connected in series with its mirror image. As indicated, the magnetic field $B$ changes sign upon reflection. The variance of the conductance fluctuations in (a) is exactly four times the variance in (b). The variance in (b) is exactly two times the variance in the absence of the reflection symmetry. The exchange of time-reversal symmetry for reflection symmetry explains the insensitivity to a magnetic field of the conductance fluctuations in a NS junction. After Brouwer and Beenakker (1995c).

$$
\operatorname{Var} G_{\mathrm{NS}}(\text { no TRS })=4 \operatorname{Var} G_{\mathrm{NN}}(\mathrm{RS}, \text { no TRS }) .
$$

The system NN is special because it possesses a reflection symmetry. Breaking reflection symmetry amounts to the replacement of the mirror-imaged segment by a different segment, with scattering matrix $s_{0}^{\prime}$ that is independent of $s_{0}$ but drawn from the same ensemble. This reduces the variance of the conductance fluctuations by a factor of two, regardless of whether time-reversal symmetry is present or not,

$$
\operatorname{Var} G_{\mathrm{NN}}(\mathrm{RS})=2 \operatorname{Var} G_{\mathrm{NN}}(\text { no RS }) \text {. }
$$

One may check this relation by an explicit calculation, but it is intuitively obvious if one considers that the eigenstates separate into even and odd states that fluctuate independently. Since breaking time-reversal symmetry by itself reduces the variance of $G_{\mathrm{NN}}$ by a factor of two, we may write

$$
\operatorname{Var} G_{\mathrm{NN}}(\mathrm{RS}, \text { no TRS })=\operatorname{Var} G_{\mathrm{NN}}(\mathrm{TRS}, \text { no RS }) \text {. }
$$

Equations (282)-(284) are exact, for any distribution of the scattering matrix that depends only on the transmission eigenvalues. We need one more relationship, which is approximate and holds only for the case of a disordered wire,

$$
\operatorname{Var} G_{\mathrm{NS}}(\mathrm{TRS}) \approx 4 \operatorname{Var} G_{\mathrm{NN}}(\mathrm{TRS}, \text { no RS }) \text {. }
$$

Equation (285) is approximate because the correct coefficient according to Eq. (280) is 4.3 and not 4. Taken together, Eqs. (282)-(285) imply the approximate relationship $\operatorname{Var} G_{\mathrm{NS}}(\mathrm{TRS}) \approx \operatorname{Var} G_{\mathrm{NS}}($ no TRS $)$.

One can thus understand the insensitivity of the conductance fluctuations to a magnetic field as an exchange of symmetries: breaking time-reversal symmetry introduces an approximate reflection symmetry into the structure of the scattering matrix. This reflection symmetry compensates the reduction of the conductance fluctuations due to breaking of time-reversal symmetry and explains the anomalous insensitivity of the fluctuations to a magnetic field.

We conclude this subsection by mentioning the effects of a voltage and of spin-orbit scattering (Brouwer and Beenakker, 1996a). If electron-hole degeneracy (ehD) is broken by a voltage $V \gg E_{\mathrm{c}} / e$, then the NS junction is equivalent to the system NN without reflection symmetry:

$$
\operatorname{Var} G_{\mathrm{NS}}\left(\text { no ehD) }=4 \operatorname{Var} G_{\mathrm{NN}}(\text { no RS). }\right.
$$

This relationship holds regardless of whether timereversal symmetry is broken or not (see Table IV). Concerning spin-orbit scattering, we know that $\operatorname{Var} G_{N}$ is four times smaller with spin-orbit scattering than without, either because $1 / \beta=1 / 4$ instead of 1 , or because $G_{0}^{2}=\left(e^{2} / h\right)^{2}$ instead of $\left(2 e^{2} / h\right)^{2}$ (see Sec. III.B.1). The same factor of four applies to $\operatorname{Var} G_{\mathrm{NS}}$ because the relationships between the systems NS and NN, Eqs. (282)(286), hold both with and without spin-orbit scattering. 


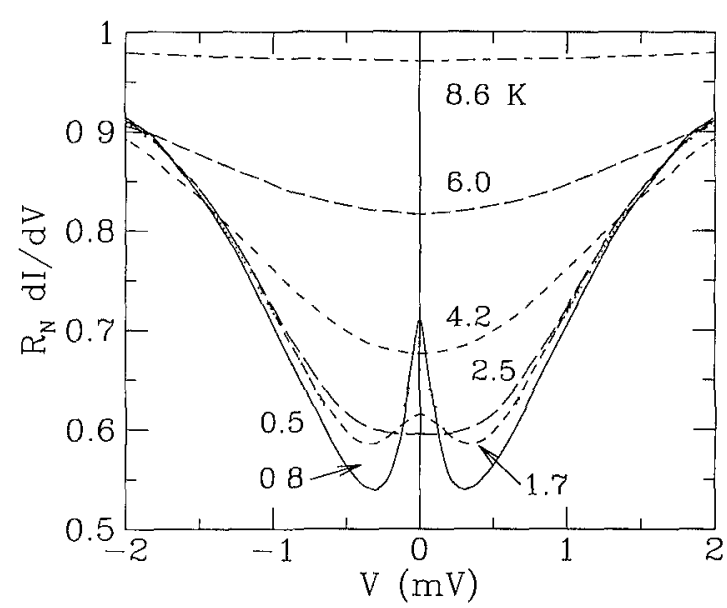

FIG. 34. Experimental data of the differential conductance $d I / d V$ (normalized by the normal-state resistance $R_{\mathrm{N}}=0.27 \Omega$ ) of a Nb-InGaAs junction, as a function of applied voltage at seven different temperatures. After Kastalsky et al. (1991).

\section{Normal-metal-superconductor junction containing a tunnel barrier}

Although the case of an ideal NS interface, considered in the previous subsection, is of considerable conceptual importance, it is more common in experiments to have a high potential barrier at the interface between the normal metal and the superconductor. The resulting interplay between normal and Andreev reflections causes a new quantum-interference effect on the conductance, as was first appreciated by Van Wees et al. (1992). The effect, now known as reflectionless tunneling, was discovered in 1991 by Kastalsky et al., as a large and narrow peak in the differential conductance of a $\mathrm{Nb}$-InGaAs junction. We reproduce their data in Fig. 34. There exists similar data from many other groups (Agraït, Rodrigo, and Vieira, 1992; Mani. Ghenim, and Theis, 1992; Nguyen, Kroemer, and Hu, 1992 ; Xiong, Xiao, and Laibowitz, 1993; Bakker et al., 1994; Lenssen et al., 1994; Magnée et al., 1994). The effect can be explained in terms of the disorder-induced opening of tunneling channels (Beenakker, Rejaei, and Melsen, 1994; Nazarov, 1994a) or equivalently as a nonequilibrium proximity effect (Volkov, Zaŭtsev, and Klapwijk, 1993; Nazarov, 1994b). To set the stage we begin by discussing the phenomenology of the effect, which gave it its name (Marmorkos, Beenakker, and Jalabert, 1993).

\section{Reflectionless tunneling}

It is instructive to first discuss the classical resistance $R_{\mathrm{NS}}^{\text {class }}$ of the NS junction. The basic approximation in $R_{\mathrm{NS}}^{\text {class }}$ is that currents rather than amplitudes are matched at the NS interface (Andreev, 1966). The result is

$$
R_{\mathrm{NS}}^{\text {class }}=\frac{h}{2 N e^{2}}\left(L / l+2 \Gamma^{-2}\right),
$$

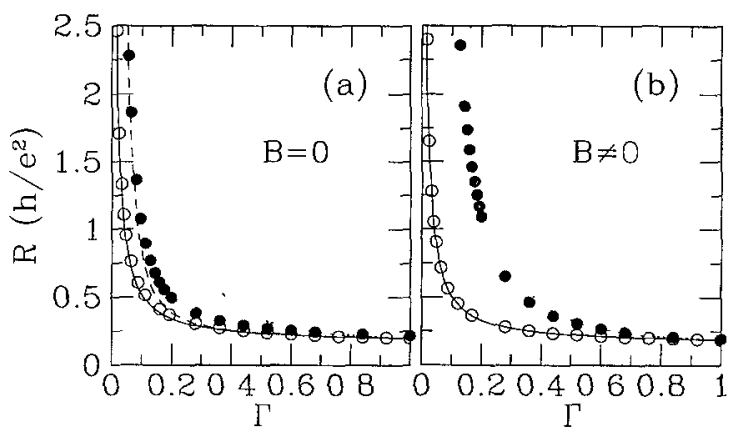

FIG. 35. Reflectionless tunneling. Filled circles: Numerically calculated resistance $R_{\mathrm{NS}}$ of a disordered NS junction, versus the transmission probability per mode $\Gamma$ of the tunnel barrier at the NS interface; open circles: resistance $R_{\mathrm{N}}$ of the same junction in the normal state; (a) is for zero magnetic field, (b) is for a flux of $10 \mathrm{~h} / \mathrm{e}$ through the disordered region. The dotted and solid curves are the classical Eqs. (287) and (288). The dashed curve is the theory of Volkov, Zaintsev, and Klapwijk (1993), which for $\Gamma \gg l / L \approx 0.12$ coincides with Eq. (289). After Marmorkos, Beenakker, and Jalabert (1993).

where $L$ is the length of the disordered region, $l$ the mean free path, $N$ the number of transverse modes, and $\Gamma \ll 1$ the tunnel probability per mode through the barrier. The contribution from the barrier to the resistance is $\propto \Gamma^{-2}$ because tunneling into a superconductor is a two-particle process (Shelankov, 1980), as both the incident electron and the Andreev-reflected hole have to tunnel through the barrier (the net result being the addition of a Cooper pair to the superconducting condensate). Equation (287) is to be contrasted with the classical resistance $R_{\mathrm{N}}^{\text {class }}$ in the normal state,

$$
R_{\mathrm{N}}^{\text {class }}=\frac{h}{2 N e^{2}}\left(L / l+\Gamma^{-1}\right),
$$

where the contribution of a resistive barrier is $\propto \Gamma^{-1}$. Let us now see how these classical results compare with the results of numerical simulations (Marmorkos, Beenakker, and Jalabert, 1993; Takane and Ebisawa, 1993).

In Fig. 35 we show the resistance (at $V=0$ ) as a function of $\Gamma$ in the absence and presence of a magnetic field. There is good agreement with the classical equations (287) and (288) for a magnetic field corresponding to 10 flux quanta through the disordered segment [Fig. $35(b)$ ]. For $B=0$, however, the situation is different [Fig. 35(a)]. The normal-state resistance (open circles) still follows approximately the classical formula (solid curve). (Deviations due to weak localization are noticeable, but small on the scale of the figure.) In contrast, the resistance of the NS junction (filled circles) lies much below the classical prediction (dotted curve). The numerical data shows that for $\Gamma \geqslant l / L$ one has approximately

$$
R_{\mathrm{NS}}(B=0, V=0) \approx R_{\mathrm{N}}^{\text {class }},
$$

which for $\Gamma \ll 1$ is much smaller than $R_{\mathrm{NS}}^{\text {class }}$. This is the phenomenon of reflectionless tunneling. In Fig. 35(a) the barrier contributes to $R_{\mathrm{NS}}$ in order $\Gamma^{-1}$, just as for 
single-particle tunneling, and not in order $\Gamma^{-2}$, as expected for two-particle tunneling. It is as if the Andreevreflected hole is not reflected by the barrier.

The numerical data of Fig. 35(a) is in good agreement with the Green's-function calculation of Volkov, Zaĭtsev, and Klapwijk (1993) (dashed curve). In the next subsection we discuss a scaling theory of reflectionless tunneling, based on the DMPK equation (Beenakker, Rejaei, and Melsen, 1994). This theory is equivalent to the Green's-function calculation but has the advantage of explicitly demonstrating how the opening of tunneling channels induces a transition from a $\Gamma^{-2}$ dependence to a $\Gamma^{-1}$ dependence as the length $L$ of the disordered region is increased beyond $l / \Gamma$.

\section{Scaling theory}

We use the parametrization $T_{n}=1 / \cosh ^{2} x_{n}$ of the transmission eigenvalues and consider the density $\rho(x, s)$ of the $x_{n}$ 's for a (dimensionless) length $s=L / l$ of the disordered region. For $s=0$, i.e., in the absence of disorder, we have the initial condition imposed by the barrier,

$$
\rho(x, 0)=N \delta\left(x-x_{0}\right)
$$

with $\Gamma=1 / \cosh ^{2} x_{0}$. The DMPK equation (157) describes how the entire distribution of the $x_{n}$ 's evolves with increasing $s$. In the large- $N$ limit, this equation reduces to the nonlinear diffusion equation (190) for the eigenvalue density (Mello and Pichard, 1989). In Sec. III.B.4 we showed how Eq. (190) can be solved exactly by a mapping onto Euler's equation of hydrodynamics. The solution is

$$
\rho(x, s)=(2 N / \pi) \operatorname{Im} U\left(x-i 0^{+}, s\right),
$$

where the complex function $U(\zeta, s)$ is determined by

$$
U(\zeta, s)=U_{0}(\zeta-s U(\zeta, s))
$$

The function $U_{0}(\zeta)$ is fixed by the initial condition (290) on $\rho$,

$$
\begin{aligned}
U_{0}(\zeta) & =\frac{\sinh 2 \zeta}{2 N} \int_{0}^{\infty} d x^{\prime} \frac{\rho\left(x^{\prime}, 0\right)}{\sinh ^{2} \zeta-\sinh ^{2} x^{\prime}} \\
& =\frac{1}{2} \sinh 2 \zeta\left(\cosh ^{2} \zeta-\Gamma^{-1}\right)^{-1} .
\end{aligned}
$$

The implicit equation (292) has multiple solutions in the entire complex plane; we need the solution for which both $\zeta$ and $\zeta-s U(\zeta, s)$ lie in the strip between the lines $y=0$ and $y=-\pi / 2$, where $\zeta=x+i y$.

The resulting density (291) is plotted in Fig. 36 (solid curves), for $\Gamma=0.1$ and several values of $s$. For $s \gg 1$ and $x \ll s$ it simplifies to

$$
\begin{aligned}
& x=\frac{1}{2} \operatorname{arccosh} \tau-\frac{1}{2} \Gamma s\left(\tau^{2}-1\right)^{1 / 2} \cos \sigma, \\
& \sigma \equiv \pi s N^{-1} \rho(x, s), \quad \tau \equiv \sigma(\Gamma s \sin \sigma)^{-1},
\end{aligned}
$$

shown by dashed curves in Fig. 36. Equation (294) agrees with the result of a Green's-function calculation by Nazarov (1994a). For $s=0$ (no disorder), $\rho$ is a delta function at $x_{0}$. On adding disorder the eigenvalue density rapidly spreads along the $x$ axis (curve a), such that

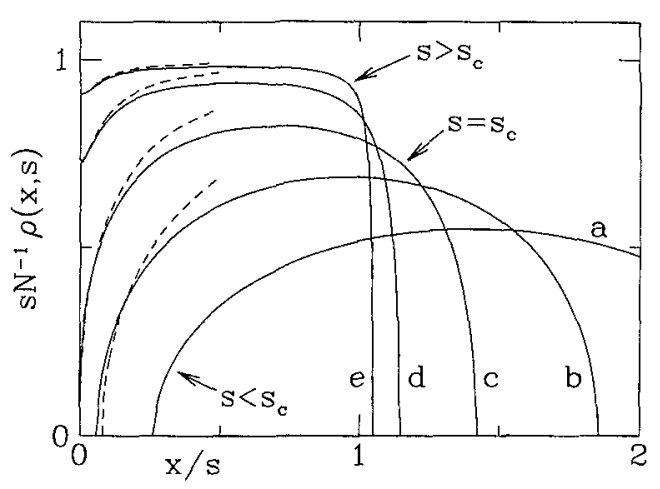

FIG. 36. Eigenvalue density $\rho(x, s)$ as a function of $x$ (in units of $s=L / l)$ for a tunnel probability per mode $\Gamma=0.1$. Curves $a, b, c, d$, and $e$ are for $s=2,4,9,30$, and 100 , respectively. The solid curves are from Eq. (291), the dashed curves from Eq. (294). The collision of the density profile with the boundary at $x=0$, for $s=s_{\mathrm{c}}=(1-\Gamma) / \Gamma$, signals the disorder-induced opening of tunneling channels responsible for the reflectionless-tunneling effect. $x$ is related to the transmission probability $T$ by $T=1 / \cosh ^{2} x$. After Beenakker, Rejaei, and Melsen (1994).

$\rho \leqslant N / s$ for $s>0$. The sharp edges of the density profile, so uncharacteristic for a diffusion profile, reveal the hydrodynamic nature of the scaling equation (190). The upper edge is at

$$
x_{\max }=s+\frac{1}{2} \ln (s / \Gamma)+\mathcal{O}(1) .
$$

Since $L / x$ has the physical significance of a localization length (see Sec. III.C.1), this upper edge corresponds to a minimum localization length $\xi_{\min }=L / x_{\max }$ of order $l$. The lower edge at $x_{\min }$ propagates from $x_{0}$ to 0 in a "time" $s_{\mathrm{c}}=(1-\Gamma) / \Gamma$. For $1 \ll s \leqslant s_{\mathrm{c}}$ one has

$$
x_{\min }=\frac{1}{2} \operatorname{arcosh}\left(s_{\mathrm{c}} / s\right)-\frac{1}{2}\left[1-\left(s / s_{\mathrm{c}}\right)^{2}\right]^{1 / 2} \text {. }
$$

It follows that the maximum localization length $\xi_{\max }=L / x_{\min }$ increases if disorder is added to a tunnel junction. This paradoxical result, that disorder enhances transmission, becomes intuitively obvious from the hydrodynamic correspondence, which implies that $\rho(x, s)$ spreads both to larger and smaller $x$ as the fictitious time $s$ progresses. When $s=s_{\mathrm{c}}$ the diffusion profile hits the boundary at $x=0$ (curve c), so that $x_{\min }=0$. This implies that for $s>s_{\mathrm{c}}$ scattering states (eigenfunctions of $t t^{\dagger}$ ) exist which tunnel through the barrier with near unit transmission probability, even if $\Gamma \ll 1$. The number $N_{\text {open }}$ of transmission eigenvalues close to one (open channels) is of the order of the number of $x_{n}$ 's in the range 0 to 1 (since $T_{n} \equiv 1 / \cosh ^{2} x_{n}$ vanishes exponentially if $x_{n}>1$ ). For $s \gg s_{\mathrm{c}}$ (curve e) we estimate

$$
N_{\text {open }} \simeq \rho(0, s)=N\left(s+\Gamma^{-1}\right)^{-1}
$$

where we have used Eq. (294). The disorder-induced opening of tunneling channels was discovered by Nazarov (1994a). It is the fundamental mechanism for the $\Gamma^{-2}$ to $\Gamma^{-1}$ transition in the conductance of an NS junction, as we now discuss.

We compare the integral equations (273) and (274) 


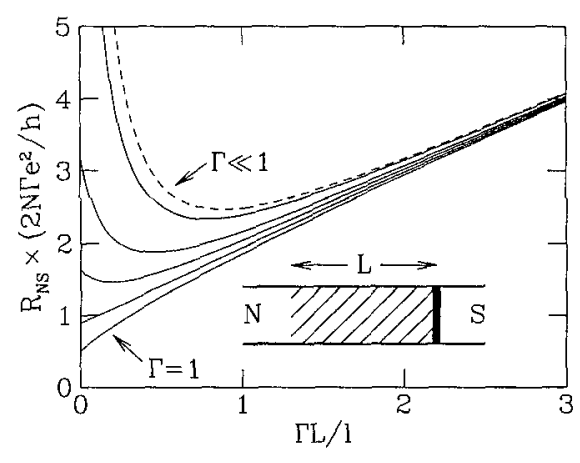

FIG. 37. Dependence of the resistance $R_{\mathrm{NS}}$ on the length $L$ of the disordered normal region (shaded in the inset), for different values of the transmittance $\Gamma$ of the normal-metalsuperconductor interface. Solid curves are computed from Eq. (299), for $\Gamma=1,0.8,0.6,0.4$, and 0.1 from bottom to top. For $\Gamma \ll 1$ the dashed curve is approached. After Beenakker, Rejaei, and Melsen (1994).

for the average conductances $\left\langle G_{\mathrm{NS}}\right\rangle$ and $\left\langle G_{\mathrm{N}}\right\rangle$. For $\Gamma \gg l / L$ one is in the regime $s \gg s_{\mathrm{c}}$ of curve e in Fig. 36 . Then the dominant contribution to the integrals over $x$ comes from the range $x / s \ll 1$, where $\rho(x, s)$ $\approx \rho(0, s)=N\left(s+\Gamma^{-1}\right)^{-1}$ is approximately independent of $x$. Substitution of $\rho(x, s)$ by $\rho(0, s)$ in Eqs. (273) and (274) yields directly

$$
\left\langle G_{\mathrm{NS}}\right\rangle \approx\left\langle G_{\mathrm{N}}\right\rangle \approx 1 / R_{\mathrm{N}}^{\text {class }}
$$

in agreement with the result (289) of the numerical simulations.

Equation (298) has the linear $\Gamma$ dependence characteristic for reflectionless tunneling. The crossover to the quadratic $\Gamma$ dependence when $\Gamma \leqslant l / L$ is obtained by evaluating the integrals (273) and (274) with the density $\rho(x, s)$ given by Eq. (291). The result is

$$
\begin{aligned}
& \left\langle G_{\mathrm{NS}}\right\rangle=\left(2 N e^{2} / h\right)\left(s+Q^{-1}\right)^{-1}, \\
& \left\langle G_{\mathrm{N}}\right\rangle=\left(2 N e^{2} / h\right)\left(s+\Gamma^{-1}\right)^{-1} .
\end{aligned}
$$

The "effective" tunnel probability $Q$ is defined by

$$
Q=\frac{\theta}{s \cos \theta}\left(\frac{\theta}{\Gamma s \cos \theta}(1+\sin \theta)-1\right),
$$

where $\theta \in(0, \pi / 2)$ is the solution of the transcendental equation

$$
\theta\left[1-\frac{1}{2} \Gamma(1-\sin \theta)\right]=\Gamma s \cos \theta
$$

For $\Gamma \ll 1$ (or $s \gg 1$ ) Eqs. (301) and (302) simplify to $Q=\Gamma \sin \theta, \theta=\Gamma s \cos \theta$, in precise agreement with the Green's-function calculation of Volkov, Zaĭtsev, and Klapwijk (1993). According to Eq. (300), the normalstate resistance increases linearly with the length $L$ of the disordered region, as expected from Ohm's law. This classical reasoning fails if one of the contacts is in the superconducting state. The scaling of the resistance $R_{\mathrm{NS}} \equiv 1 /\left\langle G_{\mathrm{NS}}\right\rangle$ with length, computed from Eq. (299), is plotted in Fig. 37. For $\Gamma=1$ the resistance increases monotonically with $L$. In the ballistic limit $L \rightarrow 0$, it equals $h / 4 N e^{2}$, half the contact resistance of a normal junction, because of Andreev reflection. For $\Gamma \leq 0.5$ a resistance minimum develops, somewhat below $L=l / \Gamma$. The resistance minimum is associated with the crossover from a quadratic to a linear dependence of $R_{\mathrm{NS}}$ on $1 / \Gamma$.

If $\Gamma s \gg 1$ one has $\theta \rightarrow \pi / 2$, hence $Q \rightarrow \Gamma$. In the opposite regime $\Gamma s \ll 1$ one has $\theta \rightarrow \Gamma s$, hence $Q \rightarrow \Gamma^{2} s$. The corresponding asymptotic expressions for $\left\langle G_{\mathrm{NS}}\right\rangle$ are, assuming $\Gamma \ll 1$ and $s \gg 1$,

$$
\begin{aligned}
& \left\langle G_{\mathrm{NS}}\right\rangle=\left(2 N e^{2} / h\right)\left(s+\Gamma^{-1}\right)^{-1}, \quad \text { if } \Gamma s \gg 1, \\
& \left\langle G_{\mathrm{NS}}\right\rangle=\left(2 N e^{2} / h\right) \Gamma^{2} s, \quad \text { if } \Gamma s \ll 1 .
\end{aligned}
$$

In either limit the conductance is greater than the classical result

$$
G_{\mathrm{NS}}^{\text {class }}=\left(2 N e^{2} / h\right)\left(s+2 \Gamma^{-2}\right)^{-1},
$$

which holds if phase coherence between electrons and holes is destroyed by a voltage or magnetic field. The peak in the conductance around $V, B=0$ is of order $\Delta G_{\mathrm{NS}}=\left\langle G_{\mathrm{NS}}\right\rangle-G_{\mathrm{NS}}^{\text {class }}$, which has the relative magnitude

$$
\frac{\Delta G_{\mathrm{NS}}}{\left\langle G_{\mathrm{NS}}\right\rangle} \approx \frac{2}{2+\Gamma^{2} s} \text {. }
$$

The scaling theory assumes zero temperature. Hekking and Nazarov $(1993,1994)$, and Zhou, Spivak, and Zyuzin (1995) have studied the conductance of a resistive NS interface at finite temperatures, when $L$ is greater than the correlation length $L_{\mathrm{c}}=\left(\hbar D / k_{\mathrm{B}} T\right)^{1 / 2}$, where $D$ is the diffusion coefficient. (This is the length scale at which the Thouless energy $E_{\mathrm{c}}$ equals the thermal energy $k_{\mathrm{B}} T$.) Their result is consistent with the limiting equation (303b), if $s=L / l$ is replaced by $L_{\mathrm{c}} / l$. The implication is that, if $L>L_{c}$, the nonlinear scaling of the resistance shown in Fig. 37 only applies to a disordered segment of length $L_{\mathrm{c}}$ adjacent to the superconductor. For the total resistance one should add the Ohmic contribution of order $\left(h / e^{2}\right)\left(L-L_{\mathrm{c}}\right) / l$ from the rest of the wire.

\section{Double-barrier junction}

In the previous subsection we have discussed how the opening of tunneling channels (i.e., the appearance of transmission eigenvalues close to one) by disorder leads to a minimum in the resistance when $L=l / \Gamma$. The minimum separates a $\Gamma^{-1}$ from a $\Gamma^{-2}$ dependence of the resistance on the transparency of the interface. We referred to the $\Gamma^{-1}$ dependence as "reflectionless tunneling," since it is as if one of the two quasiparticles which form the Cooper pair can tunnel through the barrier with probability one. In the present subsection we will show, following Melsen and Beenakker (1994), that a qualitatively similar effect occurs if the disorder in the normal region is replaced by a second tunnel barrier (tunnel probability $\Gamma^{\prime}$ ). The resistance at fixed $\Gamma$ shows a minimum as a function of $\Gamma^{\prime}$ when $\Gamma^{\prime} \simeq \Gamma$. For $\Gamma^{\prime} \leq \Gamma$ the resistance has a $\Gamma^{-1}$ dependence, so we can speak again of reflectionless tunneling. 


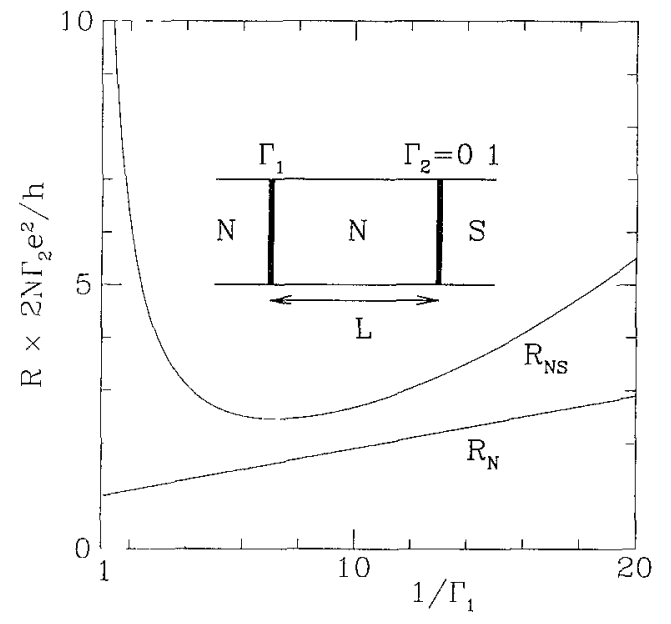

FIG. 38. Dependence of the resistances $R_{\mathrm{N}}$ and $R_{\mathrm{NS}}$ of ballistic NININ and NINIS structures, respectively, on barrier transparency $\Gamma_{1}$, while transparency $\Gamma_{2}=0.1$ is kept fixed [computed from Eqs. (307) and (308)]. The inset shows the NINIS structure considered. Here, $N$ refers to a normal metal, $I$ an insulating, and $S$ a superconducting layer. After Melsen and Beenakker (1994).

We consider an $\mathrm{NI}_{1} \mathrm{NI}_{2} \mathrm{~S}$ junction, where $\mathrm{N}=$ normal metal, $\mathrm{S}=$ superconductor, and $\mathrm{I}_{t}=$ insulator or tunnel barrier (transmission probability per mode $\left.\Gamma_{t} \equiv 1 / \cosh ^{2} \alpha_{l}\right)$. We assume ballistic motion between the barriers. (The effect of disorder is discussed later.) A straightforward calculation yields the transmission probabilities $T_{n}$ of the two barriers in series [see Eq. (243)],

$$
\begin{aligned}
& T_{n}=\left(a+b \cos \phi_{n}\right)^{-1}, \\
& a=\frac{1}{2}+\frac{1}{2} \cosh 2 \alpha_{1} \cosh 2 \alpha_{2}, \\
& b=\frac{1}{2} \sinh 2 \alpha_{1} \sinh 2 \alpha_{2},
\end{aligned}
$$

where $\phi_{n}$ is the phase accumulated between the barriers by mode $n$. We assume that $L \gg \lambda_{\mathrm{F}}$ and $N \Gamma_{l} \gg 1$, so that the conductance is not dominated by a single resonance. In this case, the phases $\phi_{n}$ are distributed uniformly in the interval $(0,2 \pi)$, and we may replace the sum over the transmission eigenvalues in Eqs. (33) and (253) by integrals over $\phi: \sum_{n=1}^{N} f\left(\phi_{n}\right) \rightarrow(N / 2 \pi) \int_{0}^{2 \pi} d \phi f(\phi)$. The result is

$$
\begin{aligned}
& G_{\mathrm{NS}}=\frac{4 N e^{2}}{h} \frac{\cosh 2 \alpha_{1} \cosh 2 \alpha_{2}}{\left(\cosh ^{2} 2 \alpha_{1}+\cosh ^{2} 2 \alpha_{2}-1\right)^{3 / 2}}, \\
& G_{\mathrm{N}}=\frac{4 N e^{2}}{h}\left(\cosh 2 \alpha_{1}+\cosh 2 \alpha_{2}\right)^{-1} .
\end{aligned}
$$

These expressions are symmetric in the indices 1 and 2, i.e., it does not matter which of the two barriers is closest to the superconductor. In the same way we can compute the entire distribution of the transmission eigenvalues, $\rho(T) \equiv \Sigma_{n} \delta\left(T-T_{n}\right) \rightarrow(N / 2 \pi) \int_{0}^{2 \pi} d \phi \delta(T-T(\phi))$. Substituting $T(\phi)=(a+b \cos \phi)^{-1}$ from Eq. (306), one finds

$$
\rho(T)=\frac{N}{\pi T}\left[b^{2} T^{2}-(a T-1)^{2}\right]^{-1 / 2} .
$$

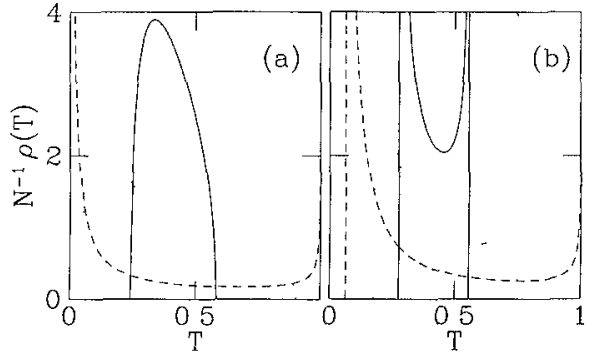

FIG. 39. Density $\rho$ of transmission eigenvalues $T$ through a normal region containing a potential barrier (transmission probability $\Gamma=0.4$ ). The left panel (a) shows the disorderinduced opening of tunneling channels (solid curve: $s=0.04$; dotted: $s=0.4$; dashed: $s=5$, where $s \equiv L / l$ ). The right panel (b) shows the opening of channels by a second tunnel barrier (transparency $\Gamma^{\prime}$; solid curve: $\Gamma^{\prime}=0.95$; dotted: $\Gamma^{\prime}=0.8$; dashed: $\Gamma^{\prime}=0.4$ ). The curves in (a) are computed from Eq. (291), the curves in (b) from Eq. (309). After Melsen and Beenakker (1994).

In Fig. 38 we plot the resistances following from Eqs. (307) and (308). Notice that $R_{\mathrm{N}}$ follows Ohm's law,

$$
R_{\mathrm{N}}=\frac{h}{2 N e^{2}}\left(1 / \Gamma_{1}+1 / \Gamma_{2}-1\right)
$$

as expected from classical considerations. In contrast, the resistance $R_{\mathrm{NS}}$ has a minimum if one of the $\Gamma$ 's is varied while keeping the other fixed. This resistance minimum cannot be explained by classical series addition of barrier resistances. If $\Gamma_{2} \ll 1$ is fixed and $\Gamma_{1}$ is varied, as in Fig. 38, the minimum occurs when $\Gamma_{1}=\sqrt{2} \Gamma_{2}$. The minimal resistance $R_{\mathrm{NS}}^{\mathrm{min}}$ is of the same order of magnitude as the resistance $R_{\mathrm{N}}$ in the normal state at the same value of $\Gamma_{1}$ and $\Gamma_{2}$. In particular, we find that $R_{\mathrm{NS}}^{\mathrm{min}}$ depends linearly on $1 / \Gamma_{l}$, whereas for a single barrier $R_{\mathrm{NS}} \propto 1 / \Gamma^{2}$.

The linear dependence on the barrier transparency shows the qualitative similarity of a ballistic NINIS junction to the disordered NIS junction considered in the previous subsection. To illustrate the similarity, we compare in Fig. 39 the densities of normal-state transmission eigenvalues. The left panel is for an NIS junction [computed using Eq. (291)], the right panel is for an NINIS junction [computed from Eq. (309)]. In the NIS junction, disorder leads to a bimodal distribution $\rho(T)$, with a peak near zero transmission and another peak near unit transmisssion (dashed curve). A similar bimodal distribution appears in the ballistic NINIS junction, for approximately equal transmission probabilities of the two barriers. There are also differences between the two cases. The NIS junction has a unimodal $\rho(T)$ if $L / l<1 / \Gamma$, while the NINIS junction has a bimodal $\rho(T)$ for any ratio of $\Gamma_{1}$ and $\Gamma_{2}$. In both cases, the opening of tunneling channels, i.e., the appearance of a peak in $\rho(T)$ near $T=1$, is the origin for the $1 / \Gamma$ dependence of the resistance.

The DMPK scaling equation can be used to investigate what happens to the resistance minimum if the region of length $L$ between the tunnel barriers contains 
impurities, with elastic mean free path $l$ (Melsen and Beenakker, 1994). In the diffusive regime $(l \ll L)$ the scaling theory is found to agree with the Green's- function calculation by Volkov, Zaŭtsev, and Klapwijk (1993) for a disordered NINIS junction. For strong barriers $\left(\Gamma_{1}, \Gamma_{2} \ll 1\right)$ and strong disorder $(L \gg l)$, one has the two asymptotic formulas

$$
\begin{aligned}
& \left\langle G_{\mathrm{NS}}\right\rangle=\frac{2 N e^{2}}{h} \frac{\Gamma_{1}^{2} \Gamma_{2}^{2}}{\left(\Gamma_{1}^{2}+\Gamma_{2}^{2}\right)^{3 / 2}}, \quad \text { if } \Gamma_{1}, \Gamma_{2} \ll l / L, \\
& \left\langle G_{\mathrm{NS}}\right\rangle=\frac{2 N e^{2}}{h}\left(L / l+1 / \Gamma_{1}+1 / \Gamma_{2}\right)^{-1}, \quad \text { if } \quad \Gamma_{1}, \Gamma_{2} \gg l / L .
\end{aligned}
$$

Equation (311a) coincides with Eq. (307) in the limit $\alpha_{1}, \alpha_{2} \gg 1$ (recall that $\Gamma_{i} \equiv 1 / \cosh ^{2} \alpha_{i}$ ). This shows that the effect of disorder on the resistance minimum can be neglected as long as the resistance of the junction is dominated by the barriers. In this case $\left\langle G_{\mathrm{NS}}\right\rangle$ depends linearly on $\Gamma_{1}$ and $\Gamma_{2}$ only if $\Gamma_{1} \approx \Gamma_{2}$. Equation (311b) shows that if the disorder dominates, $\left\langle G_{\mathrm{NS}}\right\rangle$ has a linear $\Gamma$ dependence regardless of the relative magnitude of $\Gamma_{1}$ and $\Gamma_{2}$.

The resistance minimum predicted by Eq. (307) has been observed by Takayanagi, Toyoda, and Akazaki (1996) in a junction between $\mathrm{Nb}$ and the twodimensional electron gas in an InAlAs/InGaAs heterostructure. [Similar experiments using doped GaAs instead of a heterostructure have been performed by Poirier, Mailly, and Sanquer (1996).] One of the two barriers is present naturally at the interface between $\mathrm{Nb}$ and the heterostructure. The other barrier is created electrostatically by means of a gate on top of the heterostructure, at a separation $L=0.5 \mu \mathrm{m}$ from the $\mathrm{Nb}$ interface. This separation is much less than the mean free path $l=2.8 \mu \mathrm{m}$ in the electron gas. By making the voltage $V_{\text {gatc }}$ on the gate more negative, the transparency $\Gamma_{1}$ of the tunnel barrier below the gate is reduced. The transparency of the tunnel barrier at the $\mathrm{Nb}$ interface is fixed and estimated at $\Gamma_{2}=0.7$ from the hightemperature resistance. The low-temperature resistance is plotted as a function of the gate voltage in Fig. 40 (filled circles). Also shown is the normal-state resistance (open circles), obtained by applying a voltage greater than $2 \Delta / e=3 \mathrm{mV}$ over the junction, where $\Delta$ is the energy gap of $\mathrm{Nb}$. The former has a minimum, while the latter decreases monotonically with gate voltage. A quantitative comparison with the theory needs to take into account the series resistance from a second $\mathrm{Nb}$ contact, at a distance of $3.5 \mu \mathrm{m}$ from the gate. Takayanagi et al. have found that quite a good agreement with the theoretical result (307) can be obtained.

\section{Circuit theory}

The scaling theory of reflectionless tunneling, which was the subject of Sec. IV.C.2, describes the transition from the ballistic to the diffusive regime. In the diffusive regime it is equivalent to the Green's-function theory of Volkov, Zaŭtsev, and Klapwijk (1993). A convenient for-

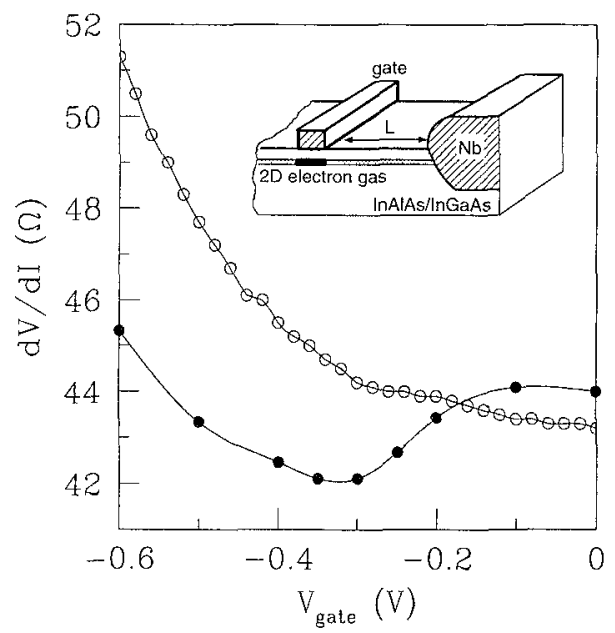

FIG. 40. Differential resistance $d V / d I$ of a gated Nb-InAlAs/ InGaAs junction as a function of the gate voltage $V_{\text {gate }}$. The more negative $V_{\text {gate }}$, the higher the tunnel barrier in the twodimensional electron gas below the gate. The gate is at a separation $L=0.5 \mu \mathrm{m}$ from the $\mathrm{Nb}$ contact (see the inset). A second $\mathrm{Nb}$ contact (not shown) is at $4 \mu \mathrm{m}$ from the first. The filled circles are for $V=0$, and the open circles are for $V>3 \mathrm{mV}$, where $V$ is the voltage between the two $\mathrm{Nb}$ contacts. (The curve through the data points is a guide to the eye.) The data for $V=0$ shows the resistance minimum expected for a ballistic double-barrier NS junction (see Fig. 38). After Takayanagi, Toyoda, and Akazaki (1996).

mulation of the Green's-function theory has been presented by Nazarov (1994b). Starting from a continuity equation for the nonequilibrium Green's function (Keldysh, 1964; Larkin and Ovchinnikov, 1975, 1977), and applying the appropriate boundary conditions, Nazarov was able to formulate a set of rules that reduce the problem of computing the resistance of an NS junction to a simple exercise in circuit theory. (An alternative derivation of these rules, using scattering matrices, has been given recently by Argaman, 1997.) The approach can be applied without further complications to multiterminal networks involving several normal and superconducting reservoirs. In this subsection we describe Nazarov's circuit theory and compare it with the results obtained from the DMPK equation.

Zero temperature is assumed, as well as infinitesimal voltage differences between the normal reservoirs (linear response). The superconducting reservoirs $S_{i}$ are all at the same voltage, because they are effectively shortcircuited by the supercurrent. The pair potential in $S_{i}$ has phase $\phi_{i}$. The reservoirs are connected by a set of diffusive normal-state conductors (length $L_{i}$, mean free path $\left.l_{i} ; s_{i} \equiv L_{i} / l_{i} \gg 1\right)$. Between the conductors there may be tunnel barriers (tunnel probability $\Gamma_{i}$ ). The presence of superconducting reservoirs has no effect on the resistance $\left(h / 2 N e^{2}\right) s_{i}$ of the diffusive conductors, but affects only the resistance $h / 2 N e^{2} \Gamma_{i}^{\text {eff }}$ of the tunnel barriers. The tunnel probability $\Gamma_{i}$ of barrier $i$ is renormalized to an effective tunnel probability $\Gamma_{i}^{\text {eff }}$, which depends on the entire circuit. 
Nazarov's rules to compute the effective tunnel probabilities are as follows. To each node and to each terminal of the circuit one assigns a vector $\vec{n}_{t}$ of unit length. For a normal reservoir, $\vec{n}_{l}=(0,0,1)$ is at the north pole, for a superconducting reservoir, $\vec{n}_{l}=\left(\cos \phi_{l}, \sin \phi_{l}, 0\right)$ is at the equator. For a node, $\vec{n}_{l}$ is somewhere on the northern hemisphere. The vector $\vec{n}_{t}$ is called a "spectral vector" because it determines the density of states. (The $z$ component of the spectral vector is the local density of states at the Fermi energy divided by the density of states in the normal reservoirs.) If the tunnel barrier is located between spectral vectors $\vec{n}_{1}$ and $\vec{n}_{2}$, its effective tunnel probability is

$$
\Gamma^{\mathrm{eff}}=\left(\vec{n}_{1} \cdot \vec{n}_{2}\right) \Gamma=\Gamma \cos \theta_{12},
$$

where $\theta_{12}$ is the angle between $\vec{n}_{1}$ and $\vec{n}_{2}$. The rule to compute the spectral vector of node $i$ follows from the continuity equation for the Green's function. Let the index $k$ label the nodes or terminals connected to node $i$ by a single tunnel barrier (with tunnel probability $\Gamma_{k}$ ). Let the index $q$ label the nodes or terminals connected to $i$ by a diffusive conductor (with $L / l \equiv s_{q}$ ). The spectral vectors then satisfy the sum rule

$$
\sum_{k}\left(\vec{n}_{l} \times \vec{n}_{k}\right) \Gamma_{k}+\sum_{q}\left(\vec{n}_{l} \times \vec{n}_{q}\right) \frac{\arccos \left(\vec{n}_{i} \cdot \vec{n}_{q}\right)}{s_{q} \sqrt{1-\left(\vec{n}_{l} \cdot \vec{n}_{q}\right)^{2}}}=0 .
$$

This is a sum rule for a set of vectors perpendicular to $\vec{n}_{l}$ of magnitude $\Gamma_{k} \sin \theta_{l k}$ or $\theta_{l q} / s_{q}$, depending on whether the element connected to node $i$ is a tunnel barrier or a diffusive conductor. There is a sum rule for each node, and together the sum rules determine the spectral vectors of the nodes.

These rules can be readily generalized (Nazarov, 1995a) to include the case that a tunnel barrier is replaced by a ballistic point contact (conductance $\left.2 N e^{2} / h\right)$. Instead of the effective tunnel probability (312) one then has the effective number of modes

$$
N^{\mathrm{eff}}=\frac{2 N}{1+\left(\vec{n}_{1} \cdot \vec{n}_{2}\right)}=N \cos ^{-2} \frac{1}{2} \theta_{12} .
$$

The corresponding replacement in the sum rule (313) is

$$
\left(\vec{n}_{\imath} \times \vec{n}_{k}\right) \Gamma_{k} \rightarrow \frac{2\left(\vec{n}_{t} \times \vec{n}_{k}\right) N_{k}}{1+\left(\vec{n}_{\imath} \cdot \vec{n}_{k}\right)} .
$$

A further generalization, to include the effect of an Aharonov-Bohm ring, has been given by Stoof and Nazarov (1996b).

As a simple example, let us consider the system of Sec. IV.C.2, consisting of one normal terminal (N), one superconducting terminal (S), one node (labeled $\mathrm{A}$ ), and two elements: a diffusive conductor (with $L / l \equiv s$ ) between $\mathrm{N}$ and $\mathrm{A}$, and a tunnel barrier (tunnel probability $\Gamma$ ) between $A$ and $S$ (see Fig. 41). There are three spectral vectors, $\vec{n}_{\mathrm{N}}, \vec{n}_{\mathrm{S}}$, and $\vec{n}_{\mathrm{A}}$. All spectral vectors lie in one plane. (This holds for any network with a single

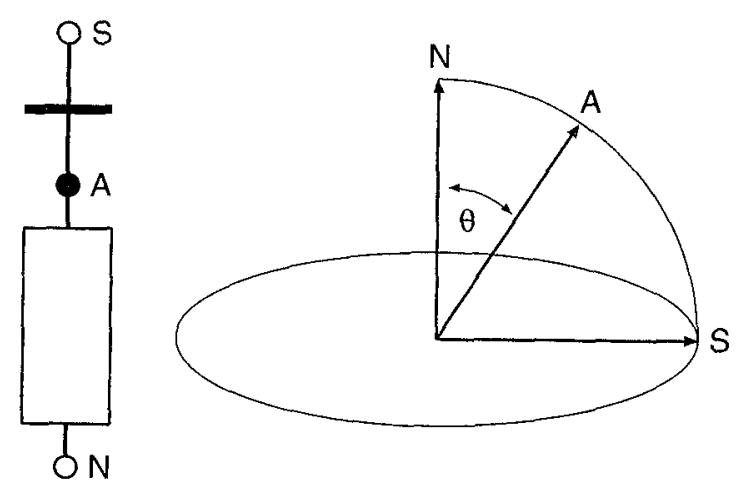

FIG. 41. Representation of a circuit by spectral vectors. At left: circuit containing two terminals (open circles), one node (filled circle), and two elements-a diffusive conductor (dotted) and a tunnel barrier (black). At right: Spectral vectors associated with the terminals N,S and with the node A.

superconducting terminal.) The resistance of the circuit is given by $R=\left(h / 2 N e^{2}\right)\left(s+1 / \Gamma^{\mathrm{eff}}\right)$, with the effective tunnel probability

$$
\Gamma^{\mathrm{eff}}=\Gamma \cos \theta_{\mathrm{AS}}=\Gamma \sin \theta .
$$

Here $\theta \in[0, \pi / 2]$ is the polar angle of $\vec{n}_{\mathrm{A}}$. This angle is determined by the sum rule (313), which in this case takes the form

$$
\Gamma \cos \theta-\theta / s=0 .
$$

Comparison with Sec. IV.C. 2 shows that $\Gamma^{\mathrm{eff}}$ coincides with the effective tunnel probability $Q$ of Eq. (301) in the limit $s \gg 1$, i.e., if one restricts oneself to the diffusive regime. That is the basic requirement for the application of the circuit theory.

Let us now consider the "fork junction" of Fig. 42, with one normal terminal $(\mathrm{N})$ and two superconducting terminals $S_{1}$ and $S_{2}$ (phases $\phi_{1} \equiv-\phi / 2$ and $\phi_{2} \equiv \phi / 2$ ). There is one node (A), which is connected to $\mathrm{N}$ by a diffusive conductor $(L / l \equiv s)$, and to $\mathrm{S}_{1}$ and $\mathrm{S}_{2}$ by tunnel barriers $\left(\Gamma_{1}\right.$ and $\left.\Gamma_{2}\right)$. This structure was studied theoretically by Hekking and Nazarov (1993) and experimentally by Pothier et al. (1994) and Dimoulas et al.

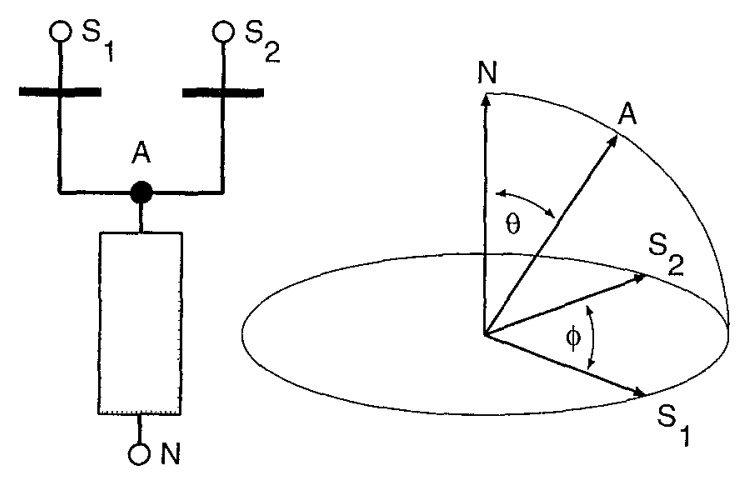

FIG. 42. Circuit diagram and spectral vectors for a structure containing one normal and two superconducting terminals (phase difference $\phi$ ). 


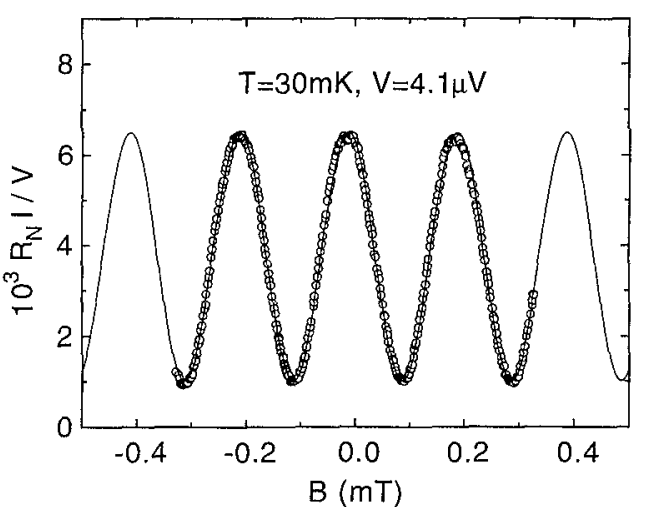

FIG. 43. Conductance of a fork junction as a function of magnetic field, which shows the dependence on the phase difference $\phi$ of the superconductor at two tunnel barriers. The circles are measurements by Pothier et al. (1994) of the current $I$ through a $\mathrm{Cu}$ wire connected to an oxidized Al fork (normalstate resistance $R_{\mathrm{N}}=1.56 \mathrm{k} \Omega$ ). The applied voltage $V$ is sufficiently low that $I / V$ is close to the linear-response conductance. (The amplitude of the oscillations at $V=0$ is $3.94 \times 10^{-6} \Omega^{-1}$, somewhat larger than in the figure.) The solid curve is a cosine fit to the data. The offset of maximum conductance from $B=0$ is attributed to a small residual field in the cryostat. After Pothier et al. (1994).

(1995). For simplicity, let us assume two identical tunnel barriers $\Gamma_{1}=\Gamma_{2} \equiv \Gamma$. Then the spectral vector $\vec{n}_{\mathrm{A}}=(\sin \theta, 0, \cos \theta)$ of node A lies symmetrically between the spectral vectors of terminals $S_{1}$ and $S_{2}$. The sum rule (313) now takes the form

$$
2 \Gamma\left|\cos \frac{1}{2} \phi\right| \cos \theta-\theta / s=0 .
$$

Its solution determines the effective tunnel rate $\Gamma^{\mathrm{eff}}=\Gamma\left|\cos \frac{1}{2} \phi\right| \sin \theta$ of each of the two barriers in parallel, and hence the conductance of the fork junction,

$$
G=\frac{2 N e^{2}}{h}\left[s+\frac{1}{2}\left(\Gamma\left|\cos \frac{1}{2} \phi\right| \sin \theta\right)^{-1}\right]^{-1} \text {. }
$$

Two limiting cases of Eqs. (318) and (319) are

$$
G=\frac{2 N e^{2}}{h}\left(s+\frac{1}{2} \Gamma^{-1}\left|\cos \frac{1}{2} \phi\right|^{-1}\right)^{-1}, \quad \text { if } \quad s \Gamma\left|\cos \frac{1}{2} \phi\right| \geqslant 1,
$$

$$
G=\frac{4 N e^{2}}{h} s \Gamma^{2}(1+\cos \phi), \quad \text { if } s \Gamma\left|\cos \frac{1}{2} \phi\right| \ll 1 .
$$

For $\phi=0$ (and $2 \Gamma \rightarrow \Gamma$ ) these expressions reduce to Eq. (303) for an NS junction with a single superconducting reservoir. The limit (320b) agrees with the finitetemperature result of Hekking and Nazarov (1993), if $s$ is replaced by $L_{\mathrm{c}} / l$ and a series resistance is added due to the normal segment, which is further than a correlation length from the NS interfaces.

Experimental data by Pothier et al. (1994) for the $\phi$ dependence of the conductance of a fork junction is shown in Fig. 43. The conductance of a $\mathrm{Cu}$ wire attached to an oxidized $\mathrm{Al}$ fork oscillates as a function of the applied magnetic field. The period corresponds to a flux

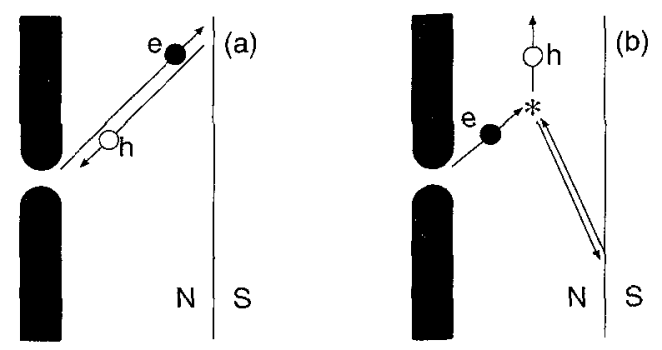

FIG. 44. Classical trajectories of an electron injected through a point contact towards superconductors, where it is Andreev reflected as a hole. In (a) the trajectory is ballistic, in (b) the electron and hole are scattered in random directions by an impurity (asterisk).

increment of $h / 2 e$ through the area enclosed by the fork and the wire and thus to $\Delta \phi=2 \pi$. The experiment is in the regime where the junction resistance is dominated by the tunnel barriers, as in Eq. (320b). [The metaloxide tunnel barriers in these structures have typically very small transmission probabilities $\Gamma \simeq 10^{-5}$, so that the regime of Eq. (320a) is not easily accessible.] Equation (320b) provides only a qualitative description of the experiment, mainly because the motion in the arms of the fork is diffusive rather than ballistic. This is why the conductance minima in Fig. 43 do not go to zero. A solution of the diffusion equation in the actual experimental geometry is required for a quantitative comparison with the theory.

\section{Normal-metal-superconductor junction containing a point contact}

Andreev reflection doubles the conductance of a point contact (Zătsev, 1980; Blonder, Tinkham, and Klapwijk, 1982; Shelankov, 1984). As illustrated in Fig. 44(a), an electron injected through the point contact is reflected back as a hole. Because electron and hole carry the same current (both in magnitude and in direction), the current through the point contact, and hence its conductance, is doubled. If $N_{0}$ is the number of transverse modes in the cross-sectional area of the point contact, then its conductance is given by

$$
G_{\mathrm{NS}}=N_{0} \frac{4 e^{2}}{h},
$$

which is twice the conductance

$$
G_{\mathrm{N}}=N_{0} \frac{2 e^{2}}{h}
$$

in the normal state (Sharvin, 1965). Equations (321) and (322) apply to ballistic transport, without scattering of the electrons by impurities. What is the effect of impurities in the region between the point contact and the superconductor? Classically, one would expect these to destroy the conductance doubling from Andreev reflection, because the hole no longer retraces the path of the electron [Fig. 44(b)]. It is therefore unlikely to find its 
way back through the point contact, so that no current doubling is to be expected. This classical picture is correct if the separation $L$ between the point contact and the NS interface is greater than the correlation length $L_{\mathrm{c}}=\left(\hbar D / k_{\mathrm{B}} T\right)^{1 / 2}$. At sufficiently low temperatures that $L \ll L_{\mathrm{c}}$, however, the conductance doubling may persist in the presence of impurity scattering (Beenakker, Melsen, and Brouwer, 1995; Golubov and Kupriyanov, 1995). To explain this effect, we first consider the angular distribution of the holes that are reflected by a disordered NS junction.

\section{Giant backscattering peak}

The angular distribution of electrons reflected by a disordered normal metal has a narrow peak at the angle of incidence. This peak has the same origin as the weaklocalization correction to the average conductance, namely the constructive interference of time-reversed sequences of multiple-scattering events (Berkovits and Feng, 1994). The peak is at most twice as high as the background. In this subsection we discuss the giant enhancement of the backscattering peak, which occurs if the normal metal is in contact with a superconductor (Beenakker, Melsen, and Brouwer, 1995). At the interface with the superconductor an electron incident from the normal metal is reflected either as an electron (normal reflection) or as a hole (Andreev reflection). Both scattering processes contribute to the backscattering peak. Normal reflection contributes a factor of two. In contrast, we will see that Andreev reflection contributes a factor $G / G_{0}$, which is $\gg 1$.

We consider a disordered normal-metal conductor which is connected at one end to a superconductor (see inset of Fig. 45). An electron at the Fermi level incident from the opposite end in mode $m$ is reflected into some other mode $n$, either as an electron or as a hole, with probability amplitudes $\left(s_{\mathrm{ee}}\right)_{n m}$ and $\left(s_{\mathrm{he}}\right)_{n m}$, respectively. The $N \times N$ matrices $s_{\text {ee }}$ and $s_{\text {he }}$ are given by Eqs. (266) and (267) (with $\varepsilon=0, \alpha=-i$ ). In terms of the polar decomposition (24) of the transmission and reflection matrices, we can write

$$
s_{\mathrm{ee}}=-2 U \frac{\sqrt{1-\mathcal{T}}}{2-\mathcal{T}} U^{\prime}, \quad s_{\mathrm{he}}=-i U^{*} \frac{\mathcal{T}}{2-\mathcal{T}} U^{\prime} .
$$

We first consider zero magnetic field $(B=0)$. Timereversal symmetry then requires that $U^{\prime}=U^{\mathrm{T}}$. We make the isotropy assumption that $U$ is uniformly distributed over the unitary group. The average over $U$ (using the formulas of Appendix B) yields

$$
\begin{aligned}
\left\langle\left|\left(s_{\mathrm{ee}}\right)_{n m}\right|^{2}\right\rangle= & \frac{\delta_{n m}+1}{N^{2}+N}\left(N-\left\langle\sum_{k} \sigma_{k}^{2}\right\rangle\right), \\
\left\langle\left|\left(s_{\mathrm{he}}\right)_{n m}\right|^{2}\right\rangle= & \frac{\delta_{n m}+1}{N^{2}+N}\left\langle\sum_{k} \sigma_{k}^{2}\right\rangle+\frac{N \delta_{n m}-1}{N^{3}-N} \\
& \times\left\langle\sum_{k \neq k^{\prime}} \sigma_{k} \sigma_{k^{\prime}}\right),
\end{aligned}
$$

where we have defined $\sigma_{k} \equiv T_{k}\left(2-T_{k}\right)^{-1}$. In the metal-

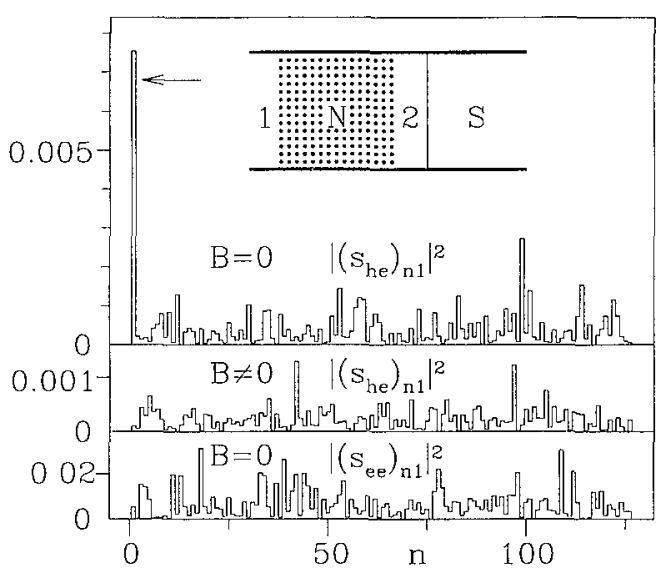

FIG. 45. Numerical simulation of a $300 \times 300$ tight-binding model for a disordered normal metal $(L=9.5 l)$, in series with a superconductor (inset). The histograms give the modal distribution for reflection of an electron at normal incidence (mode number 1). The top two panels give the distribution of reflected holes (for $B=0$ and $B=10 h / e L^{2}$ ), the bottom panel of reflected electrons (for $B=0$ ). The arrow indicates the ensemble-averaged height of the backscattering peak for Andreev reflection, predicted from Eq. (327). After Beenakker, Melsen, and Brouwer (1995). (The original figure has a mislabeled vertical axis.)

lic regime $N \gg L / l \gg 1$. In this large- $N$ limit we may factorize $\left\langle\Sigma_{k \neq k^{\prime}} \sigma_{k} \sigma_{k^{\prime}}\right\rangle$ into $\left\langle\Sigma_{k} \sigma_{k}\right\rangle^{2}$, which can be evaluated using Eq. (184):

$$
\left\langle\sum_{k} f\left(T_{k}\right)\right\rangle=\frac{N l}{L} \int_{0}^{\infty} d x f\left(1 / \cosh ^{2} x\right) .
$$

The result for normal reflection is

$$
\left\langle\left|\left(s_{\mathrm{ee}}\right)_{n m}\right|^{2}\right\rangle=\left(1+\delta_{n m}\right) \frac{1}{N}\left(1-\frac{l}{2 L}\right) .
$$

Off-diagonal $(n \neq m)$ and diagonal $(n=m)$ reflections differ by precisely a factor of two, just as in the normal state (Mello, Akkermans, and Shapiro, 1988). In contrast, for Andreev reflection we find

$$
\begin{aligned}
& \left\langle\left|\left(s_{\mathrm{he}}\right)_{n m}\right|^{2}\right\rangle=\frac{l}{2 N L} \quad(n \neq m), \\
& \left\langle\left|\left(s_{\mathrm{he}}\right)_{n n}\right|^{2}\right\rangle=\left(\frac{\pi l}{4 L}\right)^{2} .
\end{aligned}
$$

Off-diagonal and diagonal reflections now differ by an order of magnitude $N l / L \simeq G / G_{0} \gg 1$.

Equations (326) and (327) hold for $B=0$. If timereversal symmetry is broken (by a magnetic field $\left.B \gtrsim B_{\mathrm{c}} \equiv h / e L W\right)$, then the matrices $U$ and $U^{\prime}$ are independent. Carrying out the average over the unitary group in the large- $N$ limit, we find

$$
\left\langle\left|\left(s_{\mathrm{ce}}\right)_{n m}\right|^{2}\right\rangle=\frac{1}{N}\left(1-\frac{l}{2 L}\right), \quad\left\langle\left|\left(s_{\mathrm{he}}\right)_{n m}\right|^{2}\right\rangle=\frac{l}{2 N L} .
$$

Diagonal and off-diagonal reflections now occur with the same probability. 
In Fig. 45 we compare this theoretical prediction of a giant backscattering peak with a numerical simulation of the Anderson model. The results shown are raw data from a single sample. For normal reflection (bottom panel) the backscattering peak is not visible due to statistical fluctuations in the reflection probabilities (speckle noise). The backscattering peak for Andreev reflection is much larger than the fluctuations and is clearly visible (top panel). A magnetic flux of $10 \mathrm{~h} / \mathrm{e}$ through the disordered region completely destroys the peak (middle panel). The arrow in the top panel indicates the ensemble-averaged peak height from Eq. (327), consistent with the simulation within statistical fluctuations. The peak is just one mode wide, as predicted by Eq. (327). ${ }^{25}$

Coherent backscattering in the normal state is intimately related to the weak-localization correction to the average conductance. We have seen that the backscattering peak for Andreev-reflection is increased by a factor $G / G_{0}$. However, as was discussed in Sec. IV.B.2, the weak-localization correction in an NS junction remains of order $G_{0}$. The reason is that, according to Eq. (268), the conductance

$$
G_{\mathrm{NS}}=2 G_{0} \sum_{n, m}\left|\left(s_{\mathrm{he}}\right)_{n m}\right|^{2}
$$

contains the sum over all Andreev-reflection probabilities, so that the backscattering peak is averaged out. Indeed, Eqs. (327) and (328) give the same $\left\langle G_{\mathrm{NS}}\right\rangle$, up to corrections smaller by factors $1 / N$ and $l / L$. In order to observe the enhanced backscattering in a transport experiment one has to increase the sensitivity to Andreev reflection at the angle of incidence. This can be done by injecting the electrons through a point contact, as we discuss next.

\section{Conductance doubling}

The point-contact geometry is shown in the inset of Fig. 46. The point contact contains $N_{0}$ transverse modes, and the disordered region between point contact and superconductor contains $N$ transverse modes. The disordered region has length $L$ and mean free path $l$. We assume ballistic motion through the point contact, which requires that its width is much smaller than $l$. (For the opposite regime of diffusive motion through the point contact, see Volkov, 1994.) Furthermore, we assume

\footnotetext{
${ }^{25}$ The angular reflection distribution follows from the modal distribution in the large- $N$ limit. For example, in a twodimensional conductor each transverse mode $n$ is associated with angles $\pm \theta$ to the normal, such that $k_{\mathrm{F}} \sin |\theta|=n \pi / W$. One can distinguish between reflection at $\theta$ and at $-\theta$ by choosing as a new basis the sum and difference of two adjacent modes. The enhanced backscattering occurs within $\Delta \theta \simeq 2 \pi /\left(k_{\mathrm{F}} W \cos \theta\right)$ around the angle of incidence $\theta$. The decrease of $\Delta \theta$ with increasing $W$ stops when $W \simeq L$, due to the breakdown of the isotropy assumption.
}

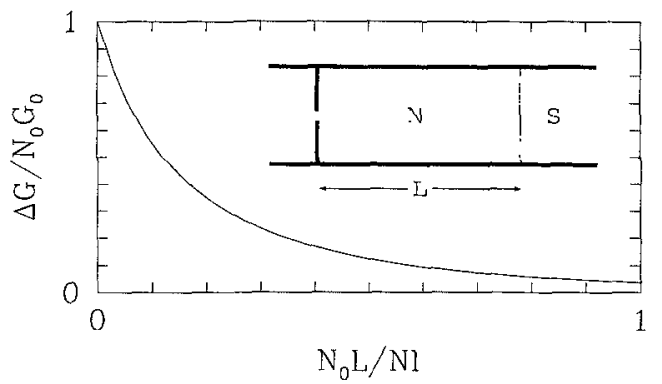

FIG. 46. Excess conductance $\Delta G=\langle G(B=0)\rangle-\left\langle G\left(B \geqslant B_{c}\right)\right\rangle$ of a point contact in series with a disordered normal-metalsuperconductor junction (inset), computed from Eqs. (331) and (333). At $B=0$ the contact conductance is twice the Sharvin conductance $N_{0} G_{0}$, provided $N_{0} L / N l \ll 1$. After Beenakker, Melsen, and Brouwer (1995).

that both $N_{0}$ and $N l / L$ are $\gg 1$, so that the conductance $G_{\text {NS }}$ of the system is much greater than the conductance quantum $G_{0} \equiv 2 e^{2} / h$.

In zero magnetic field we can compute the average conductance from Eq. (253),

$$
\left\langle G_{\mathrm{NS}}\right\rangle=2 G_{0} \int_{0}^{\infty} d x \rho(x, L) \frac{1}{\cosh ^{2} 2 x},
$$

in the parametrization $T=1 / \cosh ^{2} x$. The $L$ dependence of the density $\rho(x, L)$ follows from Eqs. (203) and (204), with the initial condition $U_{0}(\zeta)=\left(N_{0} / N\right)$ coth $\zeta$ corresponding to a point contact. The resulting conductance is

$$
\begin{aligned}
& \left\langle G_{N S}\right\rangle=G_{0}\left[\frac{1}{2}(1+\sin \theta) / N_{0}+L / N l\right]^{-1}, \\
& \frac{1}{2} \theta(1+\sin \theta)=\left(N_{0} L / N l\right) \cos \theta, \quad \theta \in(0, \pi / 2) .
\end{aligned}
$$

The implicit equation (331) has the two limiting solutions

$$
\left\langle G_{\mathrm{NS}}\right\rangle=\left\{\begin{array}{l}
G_{0}\left[1 /\left(2 N_{0}\right)+L / N l\right]^{-1} \quad \text { if } N_{0} L / N l \ll 1, \\
G_{0}\left(1 / N_{0}+L / N l\right)^{-1} \quad \text { if } N_{0} L / N l \gg 1 .
\end{array}\right.
$$

The contribution from disorder remains the same in the two limits, but the contribution from the point contact differs by a factor of two.

These results hold in zero magnetic field. A magnetic field $B$ greater than $B_{\mathrm{c}}=h /(e L W)$ effectively breaks time-reversal symmetry. Instead of Eqs. (331) and (332) one then has

$$
\left\langle G_{\mathrm{NS}}\right\rangle=G_{0}\left(1 / N_{0}+L / N l\right)^{-1},
$$

for $1 \ll N_{0} \ll N$ and $l \ll L \ll N l$, but regardless of the ratio $N_{0} L / N l$. Equation (333) is just the classical addition in series of the Sharvin conductance $N_{0} G_{0}$ of the point contact and the Drude conductance $(N l / L) G_{0}$ of the disordered region. Equation (333) applies if a voltage $V$ greater than the Thouless energy $E_{\mathrm{c}}=\hbar D / L^{2}$ breaks the electron-hole degeneracy. If both $B \ll B_{\mathrm{c}}$ and $e V \ll E_{\mathrm{c}}$, in contrast, the contribution from the point contact depends on the ratio $N_{0} L / N l$, according to Eq. (332). 


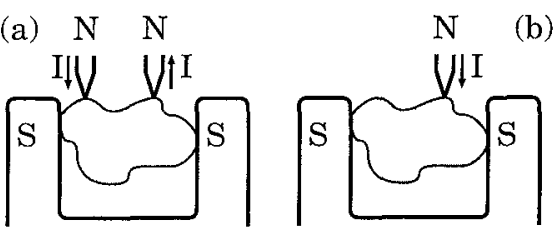

FIG. 47. Chaotic Josephson junction in a (a) four-terminal and (b) three-terminal configuration. The three-terminal configuration is equivalent as a circuit to the fork junction of Fig. 42. $N$ refers to a normal metal, $S$ a superconductor, and $I$ the current. After Brouwer and Beenakker (1996b).

In Fig. 46 we show the difference $\Delta G$ $=\left\langle G_{\mathrm{NS}}(B=0)\right\rangle-\left\langle G_{\mathrm{NS}}\left(B \gtrsim B_{\mathrm{c}}\right)\right\rangle$ of Eqs. (331) and (333). If $N_{0} / N \ll l / L \ll 1$ the conductance drops from $2 N_{0} G_{0}$ to $N_{0} G_{0}$ upon breaking time-reversal symmetry. As discussed at the beginning of this subsection, a doubling of the contact conductance at $B=0$ is a classical effect in ballistic NS junctions $(l \gg L)$ : an electron injected towards the superconductor is reflected back as a hole, doubling the current through the point contact. We now understand that the conductance doubling can survive multiple scattering in a diffusive junction $(l \ll L)$, because of the enhanced backscattering at the angle of incidence. The difference between ballistic and diffusive junctions appears in the width of the conductance peak around $B, V=0$. For a ballistic junction the width in magnetic field is $m v_{\mathrm{F}} / e L$ (determined by the curvature of the electron trajectories), and the width in voltage is the superconducting energy gap $\Delta$. These values are much greater than the values $B_{\mathrm{c}}$ and $E_{\mathrm{c}}$ for a diffusive junction. Experiments on the conductance doubling have been done by Van Son, Van Kempen, and Wyder (1987, 1988). The anomalously narrow conductance peak reported in their 1988 paper may well be due to the effects of disorder discussed here.

\section{E. Chaotic Josephson junction}

A Josephson junction is a weak link between two superconductors. The weak link could be a tunnel barrier, a point contact, or a piece of normal metal. (For a review of Josephson junctions, see Likharev, 1979.) In this subsection we will consider the special case that the weak link consists of a small (phase-coherent) metal grain (a "quantum dot"). A random-matrix theory of induced superconductivity ("proximity effect") in such a system can be constructed, based on the assumption that the classical motion in the quantum dot is chaotic (Altland and Zirnbauer, 1996; Frahm et al., 1996; Melsen et al., 1996; Zirnbauer, 1997). A phase difference $\phi$ between the superconductors induces a current through the junction. This current flows in equilibrium. Since it is a thermodynamic property of the system, it falls outside the scope of this review (we refer to Brouwer and Beenakker, 1997b).

The problem considered here is the injection of nonequilibrium quasiparticles into the Josephson junction. The system is shown schematically in Fig. 47(a). A quan- tum dot is contacted by four ballistic point contacts (with $N_{\iota}$ modes transmitted through contact $i=1,2,3,4$ ). The classical motion in the quantum dot should be chaotic on time scales greater than $\tau_{\text {ergodic }}$, and the point contacts should be sufficiently small that the dwell time $\tau_{\text {dwell }} \gg \tau_{\text {ergodic }}$ (see Sec. II). The quantum dot forms a Josephson junction in a superconducting ring. Coupling to the two superconducting banks is via point contacts 3 and 4 (phase difference $\phi$, same voltage). Contacts 1 and 2 are connected to normal metals (voltage difference $V$ ). A current $I$ is passed between contacts 1 and 2, and one measures the conductance $G=I / V$ as a function of $\phi$.

Spivak and Khmel'nitskiǔ (1982) and Altshuler, Khmel'nitskil, and Spivak (1983) computed the ensemble average $\langle G(\phi)\rangle$ in the high-temperature regime $k_{\mathrm{B}} T \gg \hbar / \tau_{\text {dwell }}$. (For more recent work in this regime, see Zhou and Spivak, 1996.) They obtained $\pi$-periodic oscillations with an amplitude of order $G_{0}$. In experiments (e.g., by De Vegvar et al., 1994) these are obscured by $2 \pi$-periodic sample-specific fluctuations of the same order of magnitude (Altshuler and Spivak, 1987). At low temperatures the fundamental periodicity of the oscillations in $\langle G(\phi)\rangle$ doubles, and their amplitude increases to become much greater than $G_{0}$ (Zaĭtsev, 1994; Beenakker, Melsen, and Brouwer, 1995; Kadigrobov et al., 1995; Allsopp et al., 1996; Claughton, Raimondi, and Lambert, 1996; Volkov and Zaŭtsev, 1996). Experiments by Petrashov et al. (1995) (and similar measurements by Courtois et al., 1996) showed such giant conductance oscillations, but these are now believed to have been caused by the thermal effect of Sec. IV.B.1 (Nazarov and Stoof, 1996). The sample-specific fluctuations remain of order $G_{0}$ at low temperatures and have been studied experimentally (Den Hartog et al., 1996) and theoretically (Brouwer and Beenakker, 1996b).

In the first part of this subsection we review the theory of the low-temperature oscillations in the ensemble-averaged conductance. Sample-specific fluctuations at low temperatures are discussed in the second part.

\section{Average conductance}

We have discussed conductance oscillations before, in the three-terminal fork junction of Sec. IV.C.4. The four-terminal Josephson junction considered here differs from the three-terminal configuration [shown in Fig. 47(b)] in the following respect: in the three-terminal configuration the current flows from a normal-metal reservoir into a superconducting reservoir, whereas in the four-terminal configuration the current flows between two normal-metal reservoirs. The four-terminal configuration shows the phase-coherent effects in a "cleaner" way, because without phase coherence in the normal metal the superconductor would have no effect at all on the conductance. In the three-terminal configuration, in contrast, there is an effect on the conductance because of the excitation gap in the bulk superconductor, even in the absence of any phase coherence between electrons and holes in the normal metal. 
The matrices $s_{\mathrm{ee}}$ and $s_{\mathrm{he}}$ [with elements $\left(s_{\mathrm{ee}}\right)_{l, n m}$ and $\left.\left(s_{\mathrm{he}}\right)_{l y, n m}\right]$ contain the combined effect of scattering in the quantum dot (described by the matrix $S$ ) and Andreev reflection at the two contacts with the superconductor. The scattering matrix $S$ of the quantum dot has submatrices $s_{l l}$, the matrix element $s_{l l, n m}$ being the scattering amplitude from mode $m$ in contact $j$ to mode $n$ in contact $i$. By summing a series of multiple Andreev reflections we obtain for $s_{\mathrm{ee}}$ and $s_{\text {he }}$ expressions analogous to Eq. (266),

$$
\begin{aligned}
& s_{\mathrm{ee}}=a-b \Omega c^{*} \Omega^{*}\left(1+c \Omega c^{*} \Omega^{*}\right)^{-1} d, \\
& s_{\mathrm{hc}}=-i b * \Omega *\left(1+c \Omega c^{*} \Omega^{*}\right)^{-1} d,
\end{aligned}
$$

where we have abbreviated

$$
\begin{array}{ll}
a=\left(\begin{array}{ll}
s_{11} & s_{12} \\
s_{21} & s_{22}
\end{array}\right), & b=\left(\begin{array}{ll}
s_{13} & s_{14} \\
s_{23} & s_{24}
\end{array}\right), \quad c=\left(\begin{array}{ll}
s_{33} & s_{34} \\
s_{43} & s_{44}
\end{array}\right), \\
d=\left(\begin{array}{ll}
s_{31} & s_{32} \\
s_{41} & s_{42}
\end{array}\right), & \Omega=\left(\begin{array}{ll}
e^{l \phi / 2} & 0 \\
0 & e^{-\imath \phi / 2}
\end{array}\right) .
\end{array}
$$

The four-terminal generalization of Eq. (268) is (Lambert, 1991, 1993; Lambert, Hui, and Robinson, 1993)

$$
\begin{aligned}
& G / G_{0}=R_{21}^{\mathrm{ec}}+R_{21}^{\mathrm{he}}+\frac{2\left(R_{11}^{\mathrm{he}} R_{22}^{\mathrm{he}}-R_{12}^{\mathrm{he}} R_{21}^{\mathrm{he}}\right)}{R_{11}^{\mathrm{he}}+R_{22}^{\mathrm{he}}+R_{12}^{\mathrm{he}}+R_{21}^{\mathrm{he}}}, \\
& R_{l l}^{\mathrm{ec}}=\sum_{n, m}\left|\left(s_{\mathrm{ee}}\right)_{l \jmath, n m}\right|^{2}, \quad R_{l J}^{\mathrm{he}}=\sum_{n, m}\left|\left(s_{\mathrm{he}}\right)_{l \jmath, n m}\right|^{2} .
\end{aligned}
$$

We evaluate the average conductance $\langle G\rangle$ by averaging $S$ over the circular ensemble (see Sec. II.A.1). At $B=0$ this means that $S=U U^{\mathrm{T}}$ with $U$ uniformly distributed in the group $\mathcal{U}(N)$ of $N \times N$ unitary matrices $\left(N=\Sigma_{l=1}^{4} N_{t}\right)$. This is the circular orthogonal ensemble (COE). If time-reversal symmetry is broken, then $S$ itself is uniformly distributed in $\mathcal{U}(N)$. This is the circular unitary ensemble (CUE). In the CUE the average can be done analytically for any $N_{l}$ and $\phi$. The result is

$$
\langle G\rangle_{\mathrm{CUE}}=G_{0} \frac{N_{1} N_{2}}{N_{1}+N_{2}}
$$

independent of $\phi$. In the COE one can do the average numerically, by generating a large number of random matrices in $\mathcal{U}(N)$. An analytical result can be obtained for $N \gg 1$. The easiest way to do this is to use Nazarov's circuit theory, described in Sec. IV.C.4. The result for the symmetric case $N_{1}=N_{2} \gg 1, N_{3}=N_{4} \equiv \rho N_{1}$ is given by

$$
\begin{aligned}
& \langle G\rangle_{\mathrm{COE}}=\frac{N_{1} G_{0}}{1+\cos \theta}, \\
& \frac{\sin \theta+\sin ^{2} \theta \cos \frac{1}{2} \phi}{\cos \theta+\cos ^{2} \theta}=\rho \cos \frac{1}{2} \phi, \quad \theta \in(0, \pi / 2) .
\end{aligned}
$$

In Fig. 48 we show the excess conductance $\Delta G=\langle G\rangle_{\mathrm{COE}}-\langle G\rangle_{\mathrm{CUE}}$ as a function of $\phi$. For $N_{l} \geqslant 10$ the numerical finite- $N$ curves (solid) are close to the analytical large- $N$ limit (337) (dotted). The excess con-

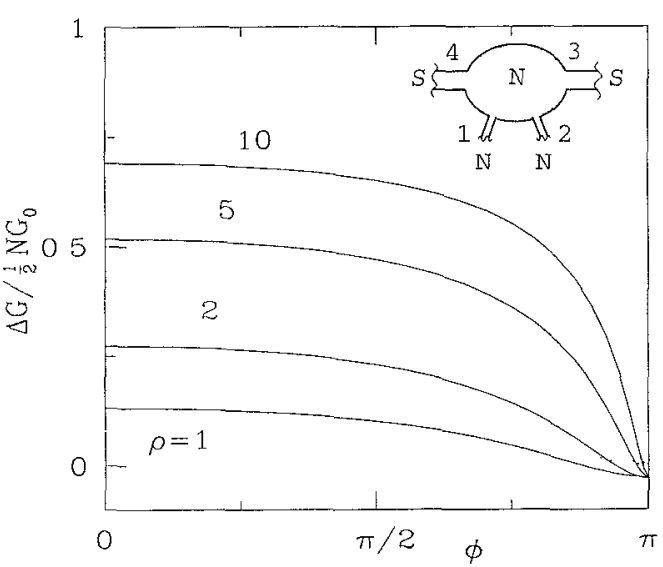

FIG. 48. Excess conductance $\Delta G=\langle G\rangle_{\mathrm{COE}}-\langle G\rangle_{\mathrm{CUE}}$ of a chaotic four-terminal Josephson junction (inset). The solid curves are computed from Eqs. (334) and (335) for $N_{1}=N_{2} \equiv N$, $N_{3}=N_{4} \equiv \rho N$, with $N=10$. The dotted curves are the large- $N$ limit given by Eq. (337). The excess conductance at $\phi=0$ is a factor $G / G_{0}=\mathcal{O}(N)$ larger than the negative weak-localization correction at $\phi=\pi$. After Beenakker, Melsen, and Brouwer (1995).

ductance $\Delta G$ is of order $N_{1} G_{0}$ and positive, except for $\phi$ close to $\pi$, where a small negative weak-localization correction of order $G_{0}$ appears.

The excess conductance is a manifestation of the giant backscattering peak of Sec. IV.D.1. To see this, note that $\left\langle R_{12}^{\text {he }}\right\rangle=\left\langle R_{21}^{\text {he }}\right\rangle,\left\langle R_{11}^{\text {he }}\right\rangle=\left\langle R_{22}^{\text {he }}\right\rangle$. (For simplicity, we assume again the symmetric case $N_{1}=N_{2}$.) Current conservation requires $R_{11}^{\mathrm{hc}}+R_{21}^{\mathrm{he}}+R_{11}^{\mathrm{ee}}+R_{21}^{\mathrm{ee}}=N_{1}$. For $N \gg 1$ we may replace $\left\langle f\left(R_{l j}\right)\right\rangle$ by $f\left(\left\langle R_{l j}\right\rangle\right)$. The average of Eq. (335) then becomes

$$
\left\langle G / G_{0}\right\rangle=\frac{1}{2} N_{1}-\frac{1}{2}\left\langle R_{11}^{\mathrm{ee}}-R_{21}^{\mathrm{ee}}\right\rangle+\frac{1}{2}\left\langle R_{11}^{\text {he }}-R_{21}^{\text {he }}\right\rangle .
$$

The first term $\frac{1}{2} N_{1}$ is the classical series conductance. The second term is the weak-localization correction due to enhanced backscattering for normal reflection. Since $\left\langle R_{11}^{\mathrm{ee}}-R_{21}^{\mathrm{ee}}\right\rangle=\mathcal{O}(1)$, this negative correction to $\frac{1}{2} N_{1}$ can be neglected if $N \gg 1$. The third term gives the excess conductance due to enhanced backscattering for Andreev reflection. Since $\left\langle R_{11}^{\text {he }}-R_{21}^{\text {he }}\right\rangle=\mathcal{O}(N)$, this positive contribution is a factor $G / G_{0}=\mathcal{O}(N)$ greater than the negative weak-localization correction.

\section{Conductance fluctuations}

The conductance of the Josephson junction contains two types of sample-specific fluctuations: aperiodic fluctuations as a function of the magnetic field $B$, and $2 \pi$-periodic fluctuations as a function of the superconducting phase difference $\phi$. To observe the fluctuations in $G(B, \phi)$, the magnetic field should be sufficiently large to break time-reversal symmetry. Otherwise the fluctuations will be obscured by the much stronger $B$ and $\phi$ dependence of the ensemble average. Den Hartog et al. (1996) have reported the experimental observation of phase-dependent magnetoconductance fluctuations in a T-shaped two-dimensional electron gas (see 

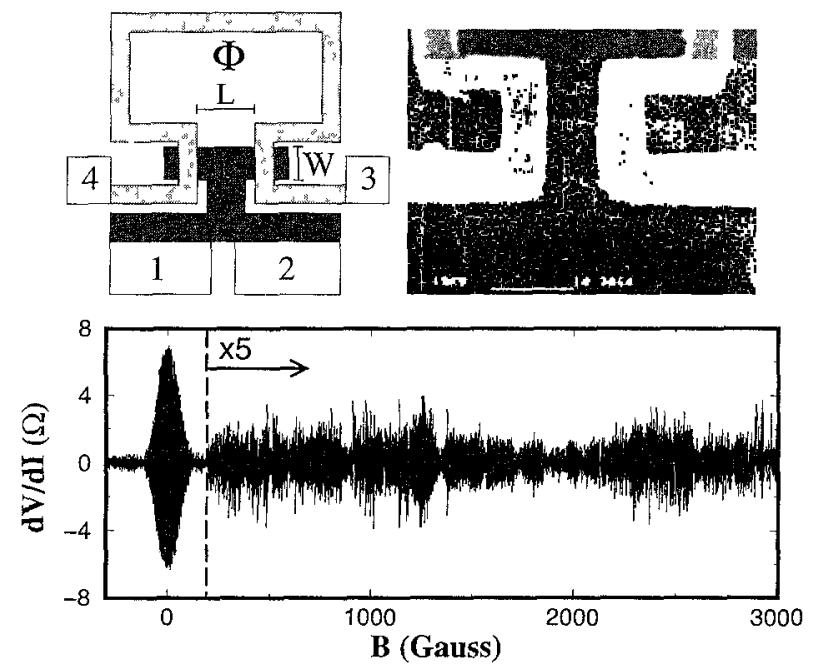

FIG. 49. Three-terminal Josephson junction. The upper-left panel shows a schematic picture of a $\mathrm{T}$-shaped $2 \mathrm{D}$ electron gas beneath a $\mathrm{Nb}$ loop. The upper-right panel shows a scanning electron micrograph of the actual device. The dimensions are $L=0.7 \mu \mathrm{m}, W=0.3 \mu \mathrm{m}$. The current $I$ flows from contacts 1 and 2 (connected to the 2D electron gas) to contacts 3 and 4 (connected to the superconducting $\mathrm{Nb}$ ). The voltage $V$ is measured from contacts 1 and 2 to 3 and 4 . The flux $\Phi \approx B \times 10.3 \mu \mathrm{m}^{2}$ through the loop determines the phase difference $\phi=(4 \pi e / h) \Phi$ of contacts 3 and 4 . The magnetoresistance oscillations at $50 \mathrm{mK}$ are plotted in the lower panel, after subtraction of a $\phi$-independent background. The amplitude near $B=0$ corresponds to a conductance of $0.1 e^{2} / h$. The amplitude for $B \geq h / e L W \equiv B_{\mathrm{c}}$ is strongly suppressed, but small oscillations remain (amplitude $\approx 0.005 e^{2} / h$ ). The envelope of these small oscillations fluctuates randomly on the scale of $B_{\text {c }}$. After Den Hartog et al. (1996).

Fig. 49). The horizontal arm of the $\mathrm{T}$ is connected to two superconductors, the vertical arm to a normal metal reservoir. The observed magnitude of the fluctuations was much smaller than $e^{2} / h$, presumably because the motion in the $\mathrm{T}$ junction was nearly ballistic. Larger fluctuations are expected if the arms of the $T$ are closed, leaving only a small opening (a point contact) for electrons to enter or leave the junction. Motion in the junction can be ballistic or diffusive. As long as it is chaotic, the statistics of the conductance fluctuations will only depend on the number of modes in the point contacts and not on the microscopic details of the junction. We review the theory of universal conductance fluctuations in a chaotic Josephson junction, following Brouwer and Beenakker (1996b).

For the conductance fluctuations there is no essential difference between the three- and four-terminal configurations of Fig. 47. We focus on the three-terminal configuration because it corresponds to the experiment of Den Hartog et al. We assume that the two point contacts to the superconductor contain $N_{3}=N_{4}$ modes each and denote by $N_{1}$ the number of modes in the contact to the normal metal. (There is only one such contact, so $N_{2} \equiv 0$.) The total number of modes in the three point contacts is $N=N_{1}+2 N_{3}$. There are two regimes, de- pending on the relative magnitude of $N_{1}$ and $N_{3}$. For $N_{1} \ll N_{3}$ the $\phi$ dependence of the conductance is strongly anharmonic. (This is the regime studied by Altshuler and Spivak, 1987.) For $N_{1} \geq N_{3}$ the oscillations are nearly sinusoidal, as observed by Den Hartog et al. The difference between the two regimes can be understood qualitatively in terms of interfering Feynman paths. In the second regime, only paths with a single Andreev reflection contribute to the conductance. Each such path depends on $\phi$ with a phase factor $e^{ \pm l \phi / 2}$. Interference of these paths yields a sinusoidal $\phi$ dependence of the conductance. In the first regime, quasiparticles undergo many Andreev reflections before leaving the junction. Hence higher harmonics appear, and the conductance becomes a random $2 \pi$-periodic function of $\phi$.

The conductance $G(B, \phi)=G_{0}(B)+G_{\phi}(B, \phi)$ consists of a $\phi$-independent background

$$
G_{0}(B)=\int_{0}^{2 \pi d \phi} \frac{d \phi}{2 \pi} G(B, \phi),
$$

plus $2 \pi$-periodic fluctuations $G_{\phi}$. In the absence of time-reversal symmetry, the ensemble average $\langle G(B, \phi)\rangle \equiv\langle G\rangle$ is independent of $B$ and $\phi$. Hence $\left\langle G_{0}(B)\right\rangle=\langle G\rangle$ and $\left\langle G_{\phi}(B, \phi)\right\rangle=0$. The correlator of $G$ is

$$
C(\delta B, \delta \phi)=\langle G(B, \phi) G(B+\delta B, \phi+\delta \phi)\rangle-\langle G\rangle^{2}
$$

Fluctuations of the background conductance are described by the correlator of $G_{0}$,

$$
\begin{aligned}
C_{0}(\delta B) & =\left\langle G_{0}(B) G_{0}(B+\delta B)\right\rangle-\langle G\rangle^{2} \\
& =\int_{0}^{2 \pi} \frac{d \delta \phi}{2 \pi} C(\delta B, \delta \phi) .
\end{aligned}
$$

(In the second equality we have used $\left\langle G_{\phi} G_{0}\right\rangle=0$.) The difference $C_{\phi}=C-C_{0}$ is the correlator of $G_{\phi}$,

$$
C_{\phi}(\delta B, \delta \phi)=\left\langle G_{\phi}(B, \phi) G_{\phi}(B+\delta B, \phi+\delta \phi)\right\rangle .
$$

For chaotic scattering without time-reversal symmetry, the scattering matrix $S$ is distributed according to the circular unitary ensemble (CUE). The CUE does not specify how $S$ at different values of $B$ is correlated. There exists a method to extend the CUE, such that it includes the parametric dependence of the scattering matrix on the magnetic field (Brouwer, 1997). The method consists of replacing the magnetic field by a time-reversal symmetry-breaking stub (see Fig. 50). This idea is similar in spirit to Büttiker's method (1986a, 1988a) of modeling inelastic scattering by a phasebreaking lead. The stub contains $N_{\text {stub }}$ modes. The end of the stub is closed, so that it conserves the number of particles without breaking phase coherence. (Buttiker's lead, in contrast, is attached to a reservoir that conserves the number of particles by matching currents, not amplitudes, and therefore breaks phase coherence.) We choose the scattering basis such that the $N_{\text {stub }} \times N_{\text {stub }}$ re- 


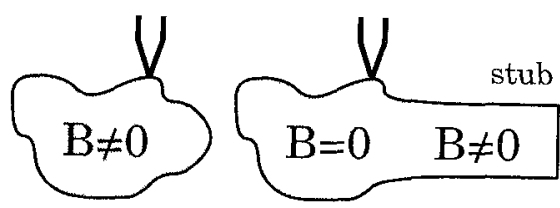

FIG. 50. Schematic picture, showing how a magnetic field can be included in the scattering-matrix ensemble. A chaotic cavity with a spatially homogeneous magnetic field (left diagram) is statistically equivalent to a chaotic cavity in zero magnetic field (right diagram), which is coupled to a closed lead (a stub) having a nonsymmetric reflection matrix. After Brouwer and Beenakker (1996b).

flection matrix $r_{\text {stub }}(B)$ of the stub equals the unit matrix at $B=0$. For nonzero magnetic fields we take

$$
r_{\text {stub }}(B)=e^{B A}, \quad a^{2} \equiv \sum_{n<m} A_{n m}^{2},
$$

where the matrix $A$ is real and antisymmetric: $A_{n m}=A_{n m}^{*}=-A_{m n}$. Particle number is conserved by the stub because $r_{\text {stub }}$ is unitary, but time-reversal symmetry is broken because $r_{\text {stub }}$ is not symmetric if $B \neq 0$. In order to model a spatially homogeneous magnetic field, it is essential that $N_{\text {stub }} \gg N$. The value of $N_{\text {stub }}$ and the precise choice of $A$ are irrelevant, all results depending only on the single parameter $a$.

The magnetic-field-dependent scattering matrix $S(B)$ in this model takes the form

$$
S(B)=U_{11}+U_{12}\left[1-r_{\text {stub }}(B) U_{22}\right]^{-1} r_{\text {stub }}(B) U_{21} \text {. }
$$

The matrices $U_{l}$ are the four blocks of a matrix $U$ representing the scattering matrix of the quantum dot at $B=0$, with the stub replaced by a regular lead. The distribution of $U$ is the circular orthogonal ensemble (COE). The distribution of $S(B)$ resulting from Eqs. (343) and (344) crosses over from the COE for $B=0$ to the CUE for $B \gg B_{\mathrm{c}}$. It is equivalent to the distribution of scattering matrices following from the Pandey-Mehta Hamiltonian (6). The parameter $a$ is related to the parameters $\alpha$ and $M$ in Eq. (6) by $B a=\alpha \sqrt{2 M}$. (The relationship between $\alpha, M$, and microscopic properties of the quantum dot was discussed in Sec. I.B.3.) The characteristic magnetic field $B_{\mathrm{c}}$ for breaking of time-reversal symmetry is $B_{\mathrm{c}}=a^{-1} \sqrt{N}$.

The correlator of the conductance can now be calculated by averaging $U$ over the COE. This can be done perturbatively if $N_{1}$ and $N_{3}$ are both $\gg 1$, for any ratio of $N_{1}$ and $N_{3}$. The result for $N_{1} \gg N_{3}$ is

$$
\begin{aligned}
& C_{0}(\delta B)=\frac{96\left(N_{3} / N_{1}\right)^{2}}{\left[1+\left(\delta B / B_{c}\right)^{2}\right]^{2}}, \\
& C_{\phi}(\delta B, \delta \phi)=\frac{1}{3} C_{0}(\delta B) \cos \delta \phi,
\end{aligned}
$$

whereas for $N_{1} \ll N_{3}$ one has

$$
C_{0}=\frac{1}{4} \sqrt{\frac{N_{1}}{N_{3}}}\left[1+\frac{2 N_{3}}{N_{1}}\left(\frac{\delta B}{B_{\mathrm{c}}}\right)^{2}\right]^{-3 / 2},
$$

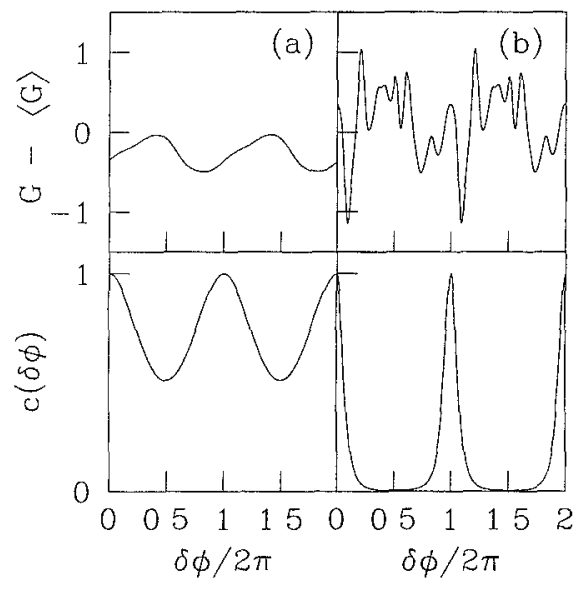

FIG. 51. Conductance fluctuations in a Josephson junction as a function of the phase difference between the superconductors, computed for a three-terminal configuration without timereversal symmetry. Top panels: Conductance minus the ensemble average (in units of $2 e^{2} / h$ ); bottom panels: normalized correlator $c(\delta \phi)=C(0, \delta \phi) / C(0,0)$, with $C(\delta B, \delta \phi)$ defined in Eq. (340). The parameters for column (a) are $N_{1}=120$, $N_{3}=N_{4}=30$; the parameters for column (b) are $N_{1}=10$, $N_{3}=N_{4}=80 .\left(N_{1}\right.$ denotes the number of modes coupling to the normal metal, and $N_{3}+N_{4}$ is the total number of modes coupling to the superconductors.) After Brouwer and Beenakker (1996b).

$$
C_{\phi}=\frac{1}{2}\left[1+\frac{2 N_{3}}{N_{1}}\left(\frac{\delta B}{B_{\mathrm{c}}}\right)^{2}+\frac{N_{3}}{4 N_{1}}(\delta \phi)^{2}\right]^{-2} .
$$

The difference between the two limiting regimes is illustrated in Fig. 51. The "sample-specific" curves in the upper panels were computed by randomly drawing a matrix $S$ from the CUE. The correlators in the lower panels were computed using large- $N$ perturbation theory. The qualitative difference between $N_{1} \geq N_{3}$ [Fig. 51(a)] and $N_{1} \ll N_{3}$ [Fig. 51(b)] is clearly visible.

\section{F. Shot noise}

If the transmission of an elementary charge $e$ can be regarded as a sequence of uncorrelated events, then the shot-noise power $P$ equals the value $2 e I \equiv P_{\text {Posson }}$ of a Poisson process (see Sec. III.E). In this subsection we discuss the enhancement of shot noise in an NS junction, following De Jong and Beenakker (1994). The enhancement originates from the fact that the current in the superconductor is carried by Cooper pairs in units of $2 e$. However, as we will see, a simple factor-of-two enhancement applies only in certain limiting cases.

In the normal state, the shot-noise power (at zero temperature and infinitesimal applied voltage) is given by (Büttiker, 1990)

$$
P_{\mathrm{N}}=P_{0} \operatorname{Tr} t t^{\dagger}\left(1-t t^{\dagger}\right)=P_{0} \sum_{n=1}^{N} T_{n}\left(1-T_{n}\right),
$$

with $P_{0} \equiv 2 e V\left(2 e^{2} / h\right)$. Closed $\left(T_{n}=0\right)$ as well as open $\left(T_{n}=1\right)$ scattering channels do not fluctuate and there- 
fore give no contribution to the shot noise. The analogue of Eq. (347) for the shot-noise power of an NS junction is

$$
\begin{aligned}
P_{\mathrm{NS}} & =4 P_{0} \operatorname{Tr} s_{\mathrm{he}} s_{\mathrm{he}}^{\dagger}\left(1-s_{\mathrm{he}} s_{\mathrm{he}}^{\dagger}\right) \\
& =P_{0} \sum_{n=1}^{N} \frac{16 T_{n}^{2}\left(1-T_{n}\right)}{\left(2-T_{n}\right)^{4}},
\end{aligned}
$$

where we have used Eq. (266) (with $\varepsilon=0$ ) to relate the scattering matrix $s_{\text {he }}$ for Andreev reflection to the transmission eigenvalues $T_{n}$ of the normal region. This requires zero magnetic field. As in the normal state, scattering channels which have $T_{n}=0$ or $T_{n}=1$ do not contribute to the shot noise. However, the way in which partially transmitting channels contribute is entirely different from the normal-state result (347).

Consider first an NS junction without disorder but with an arbitrary transmission probability $\Gamma$ per mode of the interface. In the normal state, Eq. (347) yields $P_{\mathrm{N}}=(1-\Gamma) P_{\text {Poisson }}$, implying full Poisson noise for a high tunnel barrier $(\Gamma \ll 1)$. For the NS junction we find from Eq. (348)

$$
P_{\mathrm{NS}}=P_{0} N \frac{16 \Gamma^{2}(1-\Gamma)}{(2-\Gamma)^{4}}=\frac{8(1-\Gamma)}{(2-\Gamma)^{2}} P_{\text {Poisson }},
$$

where in the second equality we have used Eq. (253). This agrees with results obtained by Khlus (1987), Muzykantskiǐ and Khmel'nitskiǐ (1994), Anantram and Datta (1996), and Martin (1996), using different methods. If $\Gamma<2(\sqrt{2}-1) \approx 0.83$, one observes a shot noise above the Poisson noise. For $\Gamma \ll 1$ one has

$$
P_{\mathrm{NS}}=4 e I=2 P_{\text {Poisson }},
$$

which is a doubling of the shot-noise power divided by the current with respect to the normal-state result. This can be interpreted as uncorrelated current pulses of $2 e$-charged particles.

Consider next an NS junction with a disordered normal region but with an ideal interface $(\Gamma=1)$. We may then apply the formula (325) for the average of a linear statistic on the transmission eigenvalues to Eqs. (253) and (348). The result is

$$
\frac{\left\langle P_{\mathrm{NS}}\right\rangle}{\left\langle G_{\mathrm{NS}}\right\rangle}=\frac{2}{3} \frac{P_{0}}{2 e^{2} / h} \Rightarrow\left\langle P_{\mathrm{NS}}\right\rangle=\frac{4}{3} e I=\frac{2}{3} P_{\text {Poisson }} .
$$

Equation (351) is twice the result in the normal state but still smaller than the Poisson noise. Corrections to Eq. (351) are of lower order in $N$ and due to quantuminterference effects.

Finally, consider an NS junction that contains a disordered normal region (length $L$, mean free path $l$ ) as well as a nonideal interface. The scaling theory of Subsection III.A. 1 can be applied to this case. Results are shown in Fig. 52, where $\left\langle P_{\text {NS }}\right\rangle / P_{\text {Poisson }}$ is plotted against $\Gamma L / l$ for various $\Gamma$. Note the crossover from the ballistic result (349) to the diffusive result (351). For a high barrier $(\Gamma \ll 1)$, the shot noise decreases from twice the Poisson noise to two-thirds the Poisson noise as the amount of disorder increases.

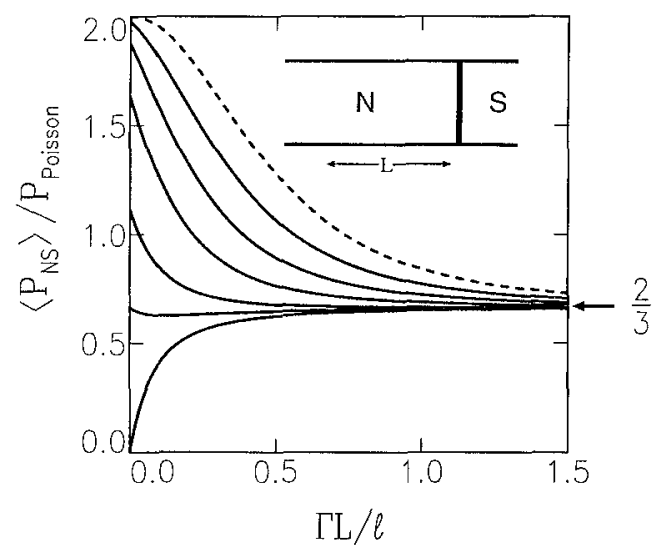

FIG. 52. The shot-noise power of a normal-metalsuperconductor junction (in units of $P_{\text {Poisson }} \equiv 2 e I$ ) as a function of the length $L$ (in units of $l / \Gamma$ ), for barrier transparencies $\Gamma=1,0.9,0.8,0.6,0.4$, and 0.2 from bottom to top. The dashed curve gives the limiting result for $\Gamma \ll 1$. For $L=0$ the noise power varies as a function of $\Gamma$ according to Eq. (349), between doubled shot noise $\left(\left\langle P_{\mathrm{NS}}\right\rangle=4 e I\right)$ for a high barrier $(\Gamma \ll 1)$ and zero in the absence of a barrier $(\Gamma=1)$. For $L \rightarrow \infty$ the noise power approaches the limiting value $\left\langle P_{\mathrm{NS}}\right\rangle=$ $\frac{4}{3} e I$ for each $\Gamma$. After De Jong and Beenakker (1994).

\section{CONCLUSION}

We conclude by identifying some open problems and directions for future research.

\section{A. Higher-dimensional geometries}

We have reviewed the random-matrix theory of quantum transport for two geometries: a quantum dot and a disordered wire. These are, essentially, zero- and onedimensional. What about higher dimensionalities? What is the statistics of the transmission eigenvalues for a twodimensional thin film or a three-dimensional cube? Here we summarize what is known.

We recall Eq. (251) for the density of transmission eigenvalues in the metallic regime,

$$
\rho_{0}(T)=\frac{N l}{2 L} \frac{1}{T \sqrt{1-T}},
$$

corresponding to the uniform density (191) in the parametrization $T=1 / \cosh ^{2} x$. [The density has a cutoff at exponentially small transmission $T \approx \exp (-2 L / l)$, which is irrelevant for transport properties.] The derivation of Sec. III.B.3 was based on the DMPK equation and hence restricted to a wire geometry (length $L$ much greater than width $W$ ). An alternative derivation by Nazarov (1994a) shows that the density (352) applies also to higher-dimensional geometries in the metallic regime. Whether or not the conductor is metallic does depend on the dimensionality. For a wire of length $L$ the condition is $L \ll N l$, while for a $L \times L$ square it is $L \ll l \exp \left(2 \pi l / \lambda_{\mathrm{F}}\right)$ (Vollhardt and Wölfle, 1992). In both cases weak disorder is assumed, meaning that the mean free path $l$ is much greater than the Fermi wavelength $\lambda_{\mathrm{F}}$. A $L \times L \times L$ cube remains in the metallic regime for 
weak disorder, regardless of how large $L$ becomes. The dimensionality independence of Eq. (352) implies, for example, that the one-third suppression of the shot noise discussed in Sec. III.E is not restricted to a wire geometry (Altshuler, Levitov, and Yakovets, 1994; Nazarov, 1994a).

A more general statement is that the nonlinear scaling equation (195) for the eigenvalue density, derived for a wire geometry, does not in fact require $L \gg W$ for its validity. It is sufficient that the conductor is in the metallic regime. We do not know of an analytical proof of this statement, but the numerical evidence for it is quite strong (Beenakker, Rejaei, and Melsen, 1994). An implication is that the scaling theory of reflectionless tunneling of Sec. IV.C.2, which is based on Eq. (195), is not restricted to a wire geometry.

Corrections $\delta \rho(T)$ to the density (352) due to weak localization are different for different dimensionalities. We recall the result (194) for a wire geometry,

$$
\begin{aligned}
\delta \rho(T)= & \frac{1}{4}(1-2 / \beta)\left[\delta\left(T-1+0^{+}\right)+2 T^{-1}\right. \\
& \left.\times(1-T)^{-1 / 2}\left(4 \ln ^{2}[\sqrt{1 / T}+\sqrt{1 / T-1}]+\pi^{2}\right)^{-1}\right],
\end{aligned}
$$

which contains a short-range (delta-function) term at unit transmission plus a long-range contribution extending down to exponentially small transmission. Nazarov (1995b) has computed $\delta \rho(T)$ for higher dimensionalities. The short-range delta-function term is always the same, but the long-range contribution depends on the geometry. This long-range contribution ensures that the weak-localization correction to the average conductance exhibits the geometry dependence known from diagrammatic perturbation theory (Lee and Ramakrishnan, 1985).

Nazarov (1996) has also shown how the geometry dependence of the variance of the conductance arises from the geometry dependence of the two-point correlation function $K\left(T, T^{\prime}\right)$. For $T, T^{\prime}$ both close to 1 the correlation function has the dimensionality-independent form

$$
\begin{aligned}
\lim _{T, T^{\prime} \rightarrow 1} K\left(T, T^{\prime}\right)= & -\frac{1}{\beta \pi^{2}} \frac{\partial}{\partial T} \frac{\partial}{\partial T^{\prime}} \\
& \times \ln \left|\frac{\sqrt{1 / T-1}-\sqrt{1 / T^{\prime}-1}}{\sqrt{1 / T-1}+\sqrt{1 / T^{\prime}-1}}\right|,
\end{aligned}
$$

corresponding to a logarithmic eigenvalue repulsion,

$$
\lim _{T, T^{\prime} \rightarrow 1} u\left(T, T^{\prime}\right)=-\ln \left|T-T^{\prime}\right|
$$

[see Eqs. (50) and (60)]. If $T$ and $T^{\prime}$ are not both close to 1 then the two-point correlation function and the interaction potential acquire contributions that depend on the dimensionality.

Nazarov's theory is a perturbation theory in the metallic regime. It requires that the conductance $G$ is much greater than $e^{2} / h$. No theory exists for the statistics of transmission eigenvalues in a square or cube geometry which extends to the insulating regime. This is the out- standing open problem of the field. So far, limited progress has been made on the extension of the DMPK equation towards higher dimensionalities (Mello and Tomsovic, 1991, 1992; Chalker and Bernhardt, 1993; Endesfelder and Kramer, 1993; Tartakovski, 1995; Endesfelder, 1996).

\section{B. Localization of interacting particles}

The interplay of interactions and localization is a formidable problem in solid-state physics. (For a review, see Belitz and Kirkpatrick, 1994.) Random-matrix techniques have given insight into the simplest case of two interacting particles (an "exciton") in a one-dimensional random potential.

Dorokhov (1990) considered a pair of harmonically bound particles moving along a disordered chain of length $L$. In the absence of disorder the total energy $2 E$ of the pair consists of kinetic energy of the center of mass plus the binding energy ${ }^{26} \quad \varepsilon_{n}=\left(n-\frac{1}{2}\right) \hbar \omega$, $n=1,2, \ldots, N$, of the harmonic interaction (frequency $\omega)$. The integer $N$ is the largest $n$ such that $\varepsilon_{n}<2 E$. The disorder potential (mean free path $l$ ) is assumed to be weak enough that it can be treated perturbatively. This requires $k l \gg N$, with $\hbar k$ the momentum of a free particle at energy $E$. Another way of stating this requirement is that the average separation $\bar{d}$ of the two particles should be much less than the mean free path.

The scattering problem of the pair, involving one propagating mode for the center-of-mass motion and $N$ bound states for the relative motion, can be mapped onto the scattering problem of a free particle with $N$ propagating modes. The probability distribution of the scattering amplitudes of the pair evolves with increasing $L$ according to a Fokker-Planck equation, analogous to the DMPK equation for a free particle. For $N=1$ the localization length $\xi_{\text {pair }}$ of the pair is of the order of the mean free path $l$, which is the single-particle localization length $\xi$ in one dimension. (The length $\xi_{\text {pair }}$ is smaller than $l$ near the ground state of the harmonic interaction and becomes larger than $l$ on approaching the first excited state.) For $N \gg 1$ the pair-localization length is greater than the mean free path by a factor $N$, analogously to Eq. (220),

$$
\xi_{\text {pair }} \simeq N l \simeq k \bar{d} \xi \text {. }
$$

The maximal enhancement $\xi_{\text {pair }} / \xi \approx k l$ is reached when $\bar{d}$ becomes comparable to $l$.

Shepelyansky (1994) studied the same problem for a weak interaction of arbitrary sign, treating the twoparticle Hamiltonian as a banded random matrix. Disorder prevents the pair to diffuse apart by more than the

\footnotetext{
${ }^{26} \mathrm{Here}$ it is assumed that the two particles are distinguishable, so that the parity of the wave function under exchange is irrelevant. If the two particles are identical, then $\varepsilon_{n}=\left(2 n-\frac{3}{2}\right) \hbar \omega$ for two bosons or for a singlet pair of electrons, while $\varepsilon_{n}=\left(2 n-\frac{1}{2}\right) \hbar \omega$ for a triplet pair of electrons.
} 
single-particle localization length, regardless of whether the interaction is attractive or repulsive. The pair localization length is greater than the single-particle localization length by a factor $\xi_{\text {pair }} / \xi \leqq k l$, independent of the sign of the interaction. This surprising result has generated a great deal of interest in the coherent propagation of correlated electron pairs. We refer to the proceedings of a recent conference for an overview (Martin, Montambaux, and Trân Thanh Vân, 1996). Much of the work is directed towards an extension of the phenomenon of interaction-assisted diffusion to spatial dimensions greater than one and to more than two particles. Whether the phenomenon is relevant for the metalinsulator transition in a disordered metal remains an open question.

\section{Localization of light}

The propagation of electromagnetic waves through a waveguide is the optical analogue of conduction through a wire. The analogy can be made more precise if the vector character of the light does not play a role, which is the case in a two-dimensional geometry with perpendicular polarization. Consider a monochromatic electric field $\overrightarrow{\mathcal{E}}(\vec{r}, t)=\hat{z} \operatorname{Re} E(x, y) \exp (i \omega t)$ (frequency $\omega$, wave number $k=\omega / c$ ), which varies only in the $x-y$ plane and is polarized in the $z$ direction. The complex scalar field $E(x, y)$ satisfies the Helmholtz equation

$$
\left[\nabla^{2}+(\omega / c)^{2} \varepsilon(x, y)\right] E(x, y)=0,
$$

with boundary condition $E=0$ at a metal surface. The (relative) dielectric constant $\varepsilon(x, y)=1+\delta \varepsilon(x, y)$ fluctuates due to disorder in the waveguide. Equation (357) is analogous to the Schrödinger equation for the wave function $\psi$ at the Fermi level of a two-dimensional electron gas. The boundary condition $\psi=0$ applies to an infinitely high potential barrier. The wavelength $\lambda=2 \pi c / \omega$ corresponds to the Fermi wavelength $\lambda_{F}$, and $(\omega / c)^{2} \delta \varepsilon(x, y)$ corresponds to the electrostatic potential $V(x, y)$ multiplied by $-2 m / \hbar^{2}$. There is also a precise correspondence between the expressions for the current density, which is $\propto \operatorname{Re} E^{*} \nabla E$ in the optical case and $\propto \operatorname{Re} \psi^{*} \nabla \psi$ in the electronic case.

The problem of localization by strong disorder is different in the two cases (John, 1984), because of the restriction $\delta \varepsilon>-1$ in the optical problem. Potentials $V$ greater than the Fermi energy have no optical analogue. As a consequence, the mean free path $l$ for light cannot be much smaller than its wavelength $\lambda$. In the case of weak disorder $l \gg \lambda$, however, optical and electronic localization are analogous. This is the relevant case for a waveguide geometry.

One new aspect of the optical problem is the absence of a conservation law if the dielectric constant has a nonzero imaginary part. The intensity of the radiation which has propagated without reflection over a distance $L$ is then multiplied by a factor $e^{\sigma L}$, with $\sigma$ negative (positive) for absorption (amplification). The growth or decay rate $\sigma$ is related to the dielectric constant by $\sigma=-2 k \operatorname{Im} \sqrt{\varepsilon}$. Absorption is present to some degree in any optical system. Amplification arises as a result of stimulated emission in a laser.

The effect of absorption or amplification on localization of light in a waveguide can be studied by a generalization of the DMPK equation. The generalization is simplest for the distribution of the reflection eigenvalues $R_{n} \equiv \lambda_{n} /\left(1+\lambda_{n}\right)$ (eigenvalues of the reflection-matrix product $\left.r r^{\dagger}\right)$. With increasing length $L$ of the waveguide, the distribution $P\left(\lambda_{1}, \lambda_{2}, \ldots, \lambda_{N}, L\right)$ evolves according to

$$
\begin{aligned}
& l \frac{\partial P}{\partial L}=\frac{2}{\beta N+2-\beta} \sum_{n=1}^{N} \frac{\partial}{\partial \lambda_{n}} \lambda_{n}\left(1+\lambda_{n}\right) \\
& \quad \times\left[J \frac{\partial}{\partial \lambda_{n}} \frac{P}{J}-\sigma l(\beta N+2-\beta) P\right], \\
& J=\prod_{i<j}^{N}\left|\lambda_{j}-\lambda_{i}\right|^{\beta} .
\end{aligned}
$$

The symmetry index $\beta$ equals 1 , unless time-reversal symmetry is broken by some magneto-optical effect. For $\sigma=0$, Eq. (358) reduces to the DMPK equation (145). The Fokker-Planck equation (358) was derived for $N=1$ (when $J \equiv 1$ ) by Gertsenshtein and Vasil'ev (1959), Kohler and Papanicolaou (1976), and Pradhan and Kumar (1994), and for $N \geqslant 1$ by Beenakker, Paasschens, and Brouwer (1996), and Bruce and Chalker (1996). The solution in the limit $L \rightarrow \infty$ is

$$
P_{\infty} \propto \prod_{i} \exp \left[\sigma l(\beta N+2-\beta) \lambda_{i}\right] \prod_{i<j}\left|\lambda_{j}-\lambda_{i}\right|^{\beta}
$$

Equation (359) holds for both positive and negative $\sigma$, but the support of $P_{\infty}$ depends on the sign of $\sigma$-all $\lambda$ 's have to be $<-1$ for $\sigma>0$ (amplification) and $>0$ for $\sigma<0$ (absorption). The distribution (359) is known in random-matrix theory as the Laguerre ensemble.

When $\sigma \neq 0$, the transmission and reflection eigenvalues are no longer related. The evolution with increasing $L$ of the $R_{n}$ 's decouples from that of the $T_{n}$ 's - but not vice versa. In fact, the evolution of the transmission eigenvalues depends not just on the reflection eigenvalues, but on all matrix elements of $r r^{\dagger}$. This is a substantial complication, and analytical progress has so far been limited to the single-mode case (Rammal and Doucot, 1987; Freilikher, Pustilnik, and Yurkevich, 1994). One exact result is the equality of the Lyapunov exponents for absorption and amplification, $\xi_{n}(\sigma)=\xi_{n}(-\sigma)$ (Paasschens, Misirpashaev, and Beenakker, 1996). [We recall that the Lyapunov exponents determine the decay of the transmission eigenvalues $T_{n} \propto \exp \left(-2 L / \xi_{n}\right)$ in the limit $L \rightarrow \infty$.] In the single-mode case, one has $\xi_{1}=2 l(1+|\sigma| l)^{-1}$ (Zhang, 1995). The multimode case has not yet been solved.

Another new aspect of the optical problem is the frequency dependence of the term $(\omega / c)^{2} \varepsilon$ in the Helmholtz equation, which for electrons would correspond to an energy-dependent potential. The frequency dependence is irrelevant for the transmission of a monochro- 
matic wave, but does affect the propagation of nonmonochromatic radiation. In particular, the velocity of propagation of a light pulse is greatly reduced near a resonance (Van Albada et al., 1991). The theory has been reviewed by Lagendijk and Van Tiggelen (1996). For a random-matrix approach to resonant multiple scattering, see Elattari, Kagalovsky, and Weidenmüller (1996).

\section{Quantum Hall effect}

The quantum Hall effect occurs in a two-dimensional electron gas in a strong perpendicular magnetic field (Prange and Girvin, 1990). (The field $B$ should be sufficiently strong that the cyclotron frequency $\omega_{\mathrm{c}}=e B / \mathrm{m}$ is much greater than the elastic-scattering rate $1 / \tau$, so that the width $\hbar / \tau$ of the Landau levels is much smaller than their spacing $\hbar \omega_{\mathrm{c}}$.) With increasing $B$, the Hall conductance decreases stepwise by $e^{2} / h$, each time a Landau level crosses the Fermi level. Each step is associated with an insulator-metal-insulator transition-the wave functions are localized on the plateaus of constant Hall conductance and extended in between the plateaus. The magnetic-field dependence of the localization length in the transition is known from numerical simulations but not yet analytically (Huckestein, 1995). An intriguing link with the random-matrix theory of a chaotic cavity has been suggested by Cobden and Kogan (1996; see also Wang, Jovanović, and Lee, 1996; Cho and Fisher, 1997).

A three-dimensional system can exhibit the quantum Hall effect if it consists of a stack of weakly coupled layers perpendicular to the magnetic field. The Bechgaard salts are a naturally occurring example. One can also grow semiconductor heterostructures containing multiple layers. This highly anisotropic system has been called a "chiral metal" (Chalker and Dohmen, 1995; Balents and Fisher, 1996; Balents, Fisher, and Zirnbauer, 1997). Conduction perpendicular to the layers occurs via overlapping states at the edges of the layers. Unlike the problem of conduction parallel to the layers, the problem of perpendicular conduction is tractable analytically. A transition from extended to localized states occurs as the number of layers is increased. Gruzberg, Read, and Sachdev (1997) have shown that this transition is governed by the DMPK equation.

\section{ACKNOWLEDGMENTS}

I am indebted to A. D. Stone for motivating me to write this review. My own research on random-matrix theory was done in collaboration with P. W. Brouwer, M. Büttiker, M. J. M. de Jong, K. M. Frahm, R. A. Jalabert, I. K. Marmorkos, J. A. Melsen, T. Sh. Misirpashaev, J. C. J. Paasschens, J.-L. Pichard, B. Rejaei, and S. A. van Langen. While writing this review, I benefitted greatly from the expert advice of P. W. Brouwer. Support by the Nederlandse organisatie voor Wetenschap- pelijk Onderzoek (NWO) and by the Stichting voor Fundamenteel Onderzoek der Materie (FOM) is gratefully acknowledged.

\section{APPENDIX A: INTEGRAL EQUATION FOR THE EIGENVALUE DENSITY}

Dyson (1972) derived the integral equation

$$
\begin{aligned}
& \int d \lambda^{\prime} \rho\left(\lambda^{\prime}\right) \ln \left|\lambda-\lambda^{\prime}\right|+\frac{1}{2}(1-2 / \beta) \ln \rho(\lambda) \\
& =V(\lambda)+\text { constant }
\end{aligned}
$$

for the eigenvalue density $\rho(\lambda)=\left\langle\Sigma_{l} \delta\left(\lambda-\lambda_{l}\right)\right\rangle$ in the Wigner-Dyson ensemble (5). The term proportional to $\ln \rho$ is an order $N^{-1} \ln N$ smaller than the other terms, and terms of still higher order in $N^{-1}$ are neglected. If the $\ln \rho$ term is neglected as well, then Eq. (A1) reduces to Wigner's integral equation (47). Equation (A1) holds for a logarithmic eigenvalue repulsion $u\left(\lambda, \lambda^{\prime}\right)$ $=-\ln \left|\lambda-\lambda^{\prime}\right|$. In this Appendix we will generalize it to a nonlogarithmic interaction.

We consider a probability distribution of the form

$$
\begin{aligned}
& P \propto e^{-\beta W}, \quad W=\sum_{l<j} u\left(\lambda_{l}, \lambda_{j}\right)+\sum_{l} V\left(\lambda_{l}\right), \\
& u\left(\lambda, \lambda^{\prime}\right)=-\ln \left|\lambda-\lambda^{\prime}\right|+\delta u\left(\lambda, \lambda^{\prime}\right),
\end{aligned}
$$

and assume that the limit $\lambda \rightarrow \lambda^{\prime}$ of $\delta u\left(\lambda, \lambda^{\prime}\right)$ exists. Note that $P$ satisfies (for each $i=1,2, \ldots, N$ ) the differential equation

$$
\frac{\partial}{\partial \lambda_{l}} P+\beta P \frac{\partial}{\partial \lambda_{l}} W=0
$$

Multiply both sides by $\delta\left(\lambda-\lambda_{l}\right)$, sum over $i$, and integrate over $\lambda_{1}, \lambda_{2}, \ldots, \lambda_{N}$. The result is

$$
\begin{aligned}
& \frac{d}{d \lambda} \rho(\lambda)+\beta \rho(\lambda) \frac{d}{d \lambda} V(\lambda)=\beta I(\lambda), \\
& I(\lambda)=-\int d \lambda^{\prime} \rho_{2}\left(\lambda, \lambda^{\prime}\right) \frac{\partial}{\partial \lambda} u\left(\lambda, \lambda^{\prime}\right) .
\end{aligned}
$$

We have defined the pair density

$$
\begin{aligned}
\rho_{2}\left(\lambda, \lambda^{\prime}\right) & \equiv\left\langle\sum_{l \neq j} \delta\left(\lambda-\lambda_{l}\right) \delta\left(\lambda^{\prime}-\lambda_{J}\right)\right\rangle \\
& =\rho(\lambda) \rho\left(\lambda^{\prime}\right)-\rho(\lambda) \delta\left(\lambda-\lambda^{\prime}\right)+K\left(\lambda, \lambda^{\prime}\right) .
\end{aligned}
$$

The two-point correlation function $K\left(\lambda, \lambda^{\prime}\right)$ was defined in Eq. (43).

We proceed with a bit of formal manipulation: 


$$
\begin{aligned}
& \int d \lambda^{\prime} \rho(\lambda) \delta\left(\lambda-\lambda^{\prime}\right) \frac{\partial}{\partial \lambda} u\left(\lambda, \lambda^{\prime}\right) \\
& =\int d \lambda^{\prime} \rho(\lambda) \delta\left(\lambda-\lambda^{\prime}\right) \frac{\partial}{\partial \lambda}\left[-\ln \left|\lambda-\lambda^{\prime}\right|+\delta u\left(\lambda, \lambda^{\prime}\right)\right] \\
& =\frac{1}{2} \rho(\lambda) \frac{d}{d \lambda} \delta u(\lambda, \lambda)-\int d \lambda^{\prime} \frac{1}{2}\left[\rho(\lambda)+\rho\left(\lambda^{\prime}\right)\right] \\
& \quad \times \frac{\delta\left(\lambda-\lambda^{\prime}\right)}{\lambda-\lambda^{\prime}} \\
& =\frac{1}{2} \rho(\lambda) \frac{d}{d \lambda} \delta u(\lambda, \lambda)+\frac{1}{2} \frac{d}{d \lambda} \rho(\lambda)
\end{aligned}
$$

Substitution into Eq. (A5) yields

$$
\begin{aligned}
\frac{I(\lambda)}{\rho(\lambda)}= & \frac{1}{2} \frac{d}{d \lambda}[\ln \rho(\lambda)+\delta u(\lambda, \lambda)] \\
& -\int d \lambda^{\prime} \rho\left(\lambda^{\prime}\right) \frac{\partial}{\partial \lambda} u\left(\lambda, \lambda^{\prime}\right) \\
& -\int d \lambda^{\prime} \frac{K\left(\lambda, \lambda^{\prime}\right)}{\rho(\lambda)} \frac{\partial}{\partial \lambda} u\left(\lambda, \lambda^{\prime}\right) .
\end{aligned}
$$

Since $K\left(\lambda, \lambda^{\prime}\right)$ is of order $N^{0}$, the last term in Eq. (A8) is a factor $N$ smaller than the other terms. We neglect this last term, substitute Eq. (A8) into Eq. (A4), divide by $\beta \rho(\lambda)$, and integrate once over $\lambda$. The result is the required generalization of Eq. (A1) to a nonlogarithmic interaction:

$$
\begin{gathered}
-\int d \lambda^{\prime} \rho\left(\lambda^{\prime}\right) u\left(\lambda, \lambda^{\prime}\right)+\frac{1}{2}(1-2 / \beta) \ln \rho(\lambda) \\
+\frac{1}{2} \delta u(\lambda, \lambda)=V(\lambda)+\text { constant. }
\end{gathered}
$$

\section{APPENDIX B: INTEGRATION OVER THE UNITARY GROUP}

Averages over the unitary group appear throughout this review. Here we collect a few results we will need repeatedly. For more extensive treatments we refer to Creutz (1978), Samuel (1980), Mello (1990), Argaman and Zee (1996), and Brouwer and Beenakker (1996a).

Let $U$ be an $N \times N$ matrix which is uniformly distributed over the group $\mathcal{U}(N)$ of $N \times N$ unitary matrices. (This is the circular unitary ensemble of Sec. II.A.1.) Averages over $\mathcal{U}(N)$ are defined as an integration with the invariant measure $d \mu(U)$,

$$
\langle f(U)\rangle=\int d \mu(U) f(U),
$$

normalized such that $\int d \mu(U)=1$. The invariance property means that

$$
\left\langle f\left(U U_{0}\right)\right\rangle=\left\langle f\left(U_{0} U\right)\right\rangle=\langle f(U)\rangle,
$$

for any fixed matrix $U_{0} \in \mathcal{U}(N)$.

The average of a polynomial function

$$
f(U)=U_{\alpha_{1} a_{1}} U_{\alpha_{2} a_{2}} \cdots U_{\alpha_{p} a_{p}} U_{\beta_{1} b_{1}}^{*} U_{\beta_{2} b_{2}}^{*} \cdots U_{\beta_{q} b_{q}}^{*}
$$

is zero unless $p=q$ and the sets $\left\{\alpha_{n}\right\}=\left\{\beta_{n}\right\}$ of left indices coincide and the sets $\left\{a_{n}\right\}=\left\{b_{n}\right\}$ of right indices coincide. The expressions for $p=1$ and 2 are

$$
\begin{aligned}
& \left\langle U_{\alpha a} U_{\beta b}^{*}\right\rangle=\frac{1}{N} \delta_{\alpha \beta} \delta_{a b}, \\
& \left\langle U_{\alpha a} U_{\alpha^{\prime} a^{\prime}} U_{\beta b}^{*} U_{\beta^{\prime} b^{\prime}}^{*}\right\rangle \\
& =\frac{1}{N^{2}-1}\left(\delta_{\alpha \beta} \delta_{a b} \delta_{\alpha^{\prime} \beta^{\prime}} \delta_{a^{\prime} b^{\prime}}+\delta_{\alpha \beta^{\prime}} \delta_{a b^{\prime}} \delta_{\alpha^{\prime} \beta^{\prime}} \delta_{a^{\prime} b}\right) \\
& \quad-\frac{1}{N\left(N^{2}-1\right)}\left(\delta_{\alpha \beta} \delta_{a b^{\prime}} \delta_{\alpha^{\prime} \beta^{\prime}} \delta_{a^{\prime} b}\right. \\
& \left.\quad+\delta_{\alpha \beta^{\prime}} \delta_{a b} \delta_{\alpha^{\prime} \beta} \delta_{a^{\prime} b^{\prime}}\right) .
\end{aligned}
$$

The leading-order term in powers of $1 / N$ in Eq. (B5) is the Gaussian approximation, which consists of replacing the real and imaginary parts of the elements of $U$ by independent Gaussian variables with zero mean and variance $1 / 2 N$. More generally, the Gaussian approximation is the leading-order term in the average

$$
\langle f(U)\rangle=N^{-p} \delta_{p q} \sum_{P} \prod_{j=1}^{p} \delta_{\alpha_{j} \beta_{P(j)}} \delta_{a_{j} b_{P(\gamma)}}+\mathcal{O}\left(N^{-p-1}\right),
$$

where the sum is over all permutations $P$ of the numbers $1,2, \ldots, p$.

APPENDIX C: HOW TO DERIVE EQ. (194) FROM EQ. (210) with

The probability distribution (210) is of the form (A2),

$$
\begin{gathered}
u\left(x, x^{\prime}\right)=-\ln \left|x-x^{\prime}\right|+\delta u\left(x, x^{\prime}\right), \\
\delta u\left(x, x^{\prime}\right)=-\frac{1}{2} \ln \left|\left(x-x^{\prime}\right)^{-1} \sinh \left(x-x^{\prime}\right)\right| \\
-\frac{1}{2} \ln \left|\left(x+x^{\prime}\right) \sinh \left(x+x^{\prime}\right)\right|, \\
V(x)=\frac{1}{2}(N-1+2 / \beta) s^{-1} x^{2}+\beta^{-1} \delta u(x, x) .
\end{gathered}
$$

(Instead of $\lambda$, we use here the variable $x \geqslant 0$.) The density $\rho(x)$ is determined to order $N^{0}$ by the integral equation (A9), which in view of Eq. (C1c) takes the form

$$
\begin{aligned}
& -\int_{0}^{\infty} d x^{\prime} \rho\left(x^{\prime}\right) u\left(x, x^{\prime}\right)+\frac{1}{2}(1-2 / \beta) \\
& \quad \times\left[\ln \rho(x)+\delta u(x, x)+s^{-1} x^{2}\right] \\
& =\frac{1}{2} N s^{-1} x^{2}+\text { constant. }
\end{aligned}
$$

We write $\rho(x)=\rho_{0}(x)+\delta \rho(x)$, with $\rho_{0}(x)$ $=N s^{-1} \theta(s-x)$ and $\delta \rho(x)$ a correction of order $N^{0}$. One can verify by substitution that $\rho_{0}$ satisfies Eq. (C2) to order $N$,

$$
-\int_{0}^{\infty} d x^{\prime} \rho_{0}\left(x^{\prime}\right) u\left(x, x^{\prime}\right)=\frac{1}{2} N s^{-1} x^{2}+\text { constant }
$$

for $s \gg 1, s \gg x$. Linearization of Eq. (C2) around $\rho_{0}$ yields an equation for $\delta \rho$, 


$$
\begin{aligned}
\int_{0}^{\infty} d x^{\prime} \delta \rho\left(x^{\prime}\right) u\left(x, x^{\prime}\right)= & \frac{1}{2}(1-2 / \beta)\left[\ln \rho_{0}(x)+\delta u(x, x)\right. \\
& \left.+s^{-1} x^{2}\right]+ \text { constant. }
\end{aligned}
$$

We substitute Eq. (C1) and extend $\rho(x)$ symmetrically to negative $x, \rho(-x) \equiv \rho(x)$. Equation (C4) becomes

$$
\begin{aligned}
& -\int_{-\infty}^{\infty} d x^{\prime} \delta \rho\left(x^{\prime}\right) \ln \left|\left(x-x^{\prime}\right) \sinh \left(x-x^{\prime}\right)\right| \\
& \quad=(1-2 / \beta)\left[\ln \rho_{0}(x)-\frac{1}{2} \ln |x \sinh (2 x)|+s^{-1} x^{2}\right]
\end{aligned}
$$

+ constant.

For $s \gg x$ the term $s^{-1} x^{2}$ may be neglected, and the term $\ln \rho_{0}$ may be absorbed into the additive constant. The remaining convolution is readily inverted by Fourier transformation,

$$
\begin{aligned}
& \delta \rho(k) \frac{2 \pi}{|k|}\left(1-e^{-\pi|k|}\right)^{-1} \\
& \quad=(1-2 / \beta) \frac{\pi}{|k|}\left(1-e^{-(1 / 2) \pi|k|}\right)^{-1} \\
& \quad \Rightarrow \delta \rho(k)=\frac{1}{2}(1-2 / \beta)\left(1+e^{-(1 / 2) \pi|k|}\right) .
\end{aligned}
$$

The inverse Fourier transform of $\delta \rho(k)$ is Eq. (194).

\section{APPENDIX D: CALCULATION OF THE WEAK-LOCALIZATION CORRECTIONS IN TABLE III}

In this Appendix we show how the weak-localization corrections $\delta G_{\mathrm{NS}}$ in a normal-metal-superconductor junction, listed in Table III, are obtained. We first consider a system without spin-orbit scattering (Brouwer and Beenakker, 1995b) and then discuss the effect of strong spin-orbit scattering (Slevin, Pichard, and Mello, 1996). The starting point of this calculation is Eq. (269). We assume that the length $L$ of the disordered normal region is much greater than the superconducting coherence length $\xi \simeq\left(\hbar v_{\mathrm{F}} l / \Delta_{0}\right)^{1 / 2}$ (with $v_{\mathrm{F}}$ the Fermi velocity and $l$ the mean free path in the normal metal). This implies that the Thouless energy $E_{\mathrm{c}} \simeq \hbar v_{\mathrm{F}} l / L^{2}$ is much smaller than the superconducting energy gap $\Delta_{0}$. In the voltage range $V \leq E_{\mathrm{c}} / e$ we may therefore assume that $e V \ll \Delta_{0}$, hence $\alpha \equiv \exp \left[-i \arccos \left(\varepsilon / \Delta_{0}\right)\right] \rightarrow-i$. Using the polar decomposition (24) of the transmission and reflection matrices, Eq. (269b) can be replaced by

$$
\begin{aligned}
m(\varepsilon)= & \sqrt{\mathcal{T}(\varepsilon)}\left[1+u(\varepsilon) \sqrt{\mathcal{R}(-\varepsilon)} u^{*}(-\varepsilon)\right. \\
& \times \sqrt{\mathcal{R}(\varepsilon)}]^{-1} u(\varepsilon) \sqrt{\mathcal{T}(-\varepsilon)}, \\
u(\varepsilon) \equiv & V^{\prime}(\varepsilon) V^{*}(-\varepsilon),
\end{aligned}
$$

where $\mathcal{T}$ is the matrix of transmission eigenvalues and $\mathcal{R}=1-\mathcal{T}$. In the presence of time-reversal symmetry, $V^{\prime}=V^{\mathrm{T}}$. If time-reversal symmetry is broken, $V$ and $V^{\prime}$ are independent. In the case of electron-hole degeneracy, the difference between $+\varepsilon$ and $-\varepsilon$ may be neglected. If electron-hole degeneracy is broken, the scattering matrices at $\pm \varepsilon$ are independent. Of the four entries in Table III, the case that both time-reversal symmetry and electron-hole degeneracy are present is the easiest, because then $u=1$ and Eq. (269) simplifies to the linear statistic (253). The result for $\delta G_{\mathrm{NS}}$ is Eq. (279). The three other entries are more difficult because we need to average over the unitary matrices as well as over the transmission eigenvalues. We will discuss the three cases separately.

\section{Broken time-reversal symmetry}

We first consider the case that electron-hole degeneracy is present but time-reversal symmetry is broken. According to the isotropy assumption in a wire geometry (see Sec. III.A.2), $V$ and $V^{\prime}$, and hence $u$, are uniformly distributed in the unitary group $\mathcal{U}(N)$. We may perform the average $\langle\cdots\rangle$ over the ensemble of scattering matrices in two steps, $\langle\cdots\rangle=\left\langle\langle\cdots\rangle_{u}\right\rangle_{\mathcal{T}}$, where $\langle\cdots\rangle_{u}$ and $\langle\cdots\rangle_{\tau}$ are, respectively, the average over the unitary matrix $u$ and over the transmission eigenvalues $T_{i}$. We compute $\langle\cdots\rangle_{u}$ by an expansion in powers of $N^{-1}$. To integrate the rational function (D1) of $u$ over $\mathcal{U}(N)$, we first expand it into a geometric series and then use the general rules for the integration of polynomials of $u$ (see Appendix B). The polynomials we need are

$$
\begin{aligned}
& \left\langle G_{\mathrm{NS}}\right\rangle_{u}=\frac{4 e^{2}}{h} \sum_{p, q=0}^{\infty}(-1)^{p+q} M_{p q}, \\
& M_{p q}=\left\langle\operatorname{Tr} \mathcal{T}\left(u \sqrt{\mathcal{R}} u^{*} \sqrt{\mathcal{R}}\right)^{p} u \mathcal{T} u^{\dagger}\left(\sqrt{\mathcal{R}} u^{\mathrm{T}} \sqrt{\mathcal{R}} u^{\dagger}\right)^{q}\right\rangle_{u} .
\end{aligned}
$$

Neglecting terms of order $N^{-1}$, we find

$$
M_{p q}=\left\{\begin{array}{l}
N \tau_{1}^{2}\left(1-\tau_{1}\right)^{2 p} \text { if } p=q, \\
\tau_{1}\left(\tau_{1}^{2}+\tau_{1}-2 \tau_{2}\right)\left(1-\tau_{1}\right)^{p+q-1} \\
-2 \min (p, q) \tau_{1}^{2}\left(\tau_{1}^{2}-\tau_{2}\right)\left(1-\tau_{1}\right)^{p+q-2} \\
\quad \text { if }|p-q| \text { odd, } \\
0 \text { if }|p-q| \text { even, and } p \neq q,
\end{array}\right.
$$

where we have defined the moment $\tau_{k}=N^{-1} \Sigma_{i} T_{i}^{k}$. The summation over $p$ and $q$ leads to

$$
\frac{h}{4 e^{2}}\left\langle G_{\mathrm{NS}}\right\rangle_{u}=\frac{N \tau_{1}}{2-\tau_{1}}-\frac{4 \tau_{1}-2 \tau_{1}^{2}+2 \tau_{1}^{3}-4 \tau_{2}}{\tau_{1}\left(2-\tau_{1}\right)^{3}} .
$$

It remains to average over the transmission eigenvalues. Since $\tau_{k}$ is a linear statistic, we know that its sample-to-sample fluctuations are order $1 / N$ smaller than the average. Hence

$$
\left\langle f\left(\tau_{k}\right)\right\rangle_{T}=f\left(\left\langle\tau_{k}\right\rangle\right)\left[1+\mathcal{O}\left(N^{-2}\right)\right],
$$

which implies that we may replace the average of the rational function (D4) of the $\tau_{k}$ 's by the rational function of the average $\left\langle\tau_{k}\right\rangle$. This average has the $1 / N$ expansion

$$
\left\langle\tau_{k}\right\rangle=\left\langle\tau_{k}\right\rangle_{0}+\mathcal{O}\left(N^{-2}\right)
$$

where $\left\langle\tau_{k}\right\rangle_{0}$ is $\mathcal{O}\left(N^{0}\right)$. There is no term of order $N^{-1}$ in 
the absence of time-reversal symmetry. From Eqs. (D4)-(D6) we obtain the $1 / N$ expansion of the average conductance,

$$
\begin{aligned}
\frac{h}{4 e^{2}}\left\langle G_{\mathrm{NS}}\right\rangle= & \frac{N\left\langle\tau_{1}\right\rangle_{0}}{2-\left\langle\tau_{1}\right\rangle_{0}}-\frac{4\left\langle\tau_{1}\right\rangle_{0}-2\left\langle\tau_{1}\right\rangle_{0}^{2}+2\left\langle\tau_{1}\right\rangle_{0}^{3}-4\left\langle\tau_{2}\right\rangle_{0}}{\left\langle\tau_{1}\right\rangle_{0}\left(2-\left\langle\tau_{1}\right\rangle_{0}\right)^{3}} \\
& +\mathcal{O}\left(N^{-1}\right) .
\end{aligned}
$$

Equation (D7) is generally valid for any distribution of the transmission eigenvalues. We apply it to the case of a disordered wire in the limit $N \rightarrow \infty, l / L \rightarrow 0$ at constant $N l / L$. The moments $\left\langle\tau_{k}\right\rangle_{0}$ are given by

$$
\left\langle\tau_{k}\right\rangle_{0}=\frac{l}{L} \int_{0}^{\infty} \frac{d x}{\cosh ^{2 k} x} \Rightarrow\left\langle\tau_{1}\right\rangle_{0}=\frac{l}{L}, \quad\left\langle\tau_{2}\right\rangle_{0}=\frac{2 l}{3 L} .
$$

Substitution into Eq. (D7) yields the weak-localization correction $\delta G_{\mathrm{NS}}=-\frac{2}{3} e^{2} / h$, see Table III.

\section{Broken electron-hole degeneracy}

If time-reversal symmetry is present but electron-hole degeneracy is broken, then one has $u^{\dagger}(-e V)=u(e V)$, with $u(e V)$ uniformly distributed in $\mathcal{U}(N)$. A calculation similar to that in the previous subsection yields for the average over $u$ :

$$
\begin{aligned}
\frac{h}{4 e^{2}}\left\langle G_{\mathrm{NS}}\right\rangle_{u}= & N \tau_{1+} \tau_{1-}\left(\tau_{1+}+\tau_{1-}-\tau_{1+} \tau_{1-}\right)^{-1} \\
& +\left(\tau_{1+}+\tau_{1-}-\tau_{1+} \tau_{1-}\right)^{-3}\left[2 \tau_{1+}^{2} \tau_{1-}^{2}\right. \\
& -\tau_{1+}^{3} \tau_{1-}^{2}-\tau_{1+}^{2} \tau_{1-}^{3}-\tau_{2+} \tau_{1-}^{2}-\tau_{1+}^{2} \tau_{2-} \\
& \left.+\tau_{2+} \tau_{1-}^{3}+\tau_{1+}^{3} \tau_{2-}\right],
\end{aligned}
$$

where we have abbreviated $\tau_{k \pm}=\tau_{k}( \pm e V)$. The next step is the average over the transmission eigenvalues. We may still use Eq. (D5), and we note that $\left\langle\tau_{k}(\varepsilon)\right\rangle \equiv\left\langle\tau_{k}\right\rangle$ is independent of $\varepsilon$. (The energy scale for variations in $\left\langle\tau_{k}(\varepsilon)\right\rangle$ is $E_{\mathrm{F}}$, which is much greater than the energy scale of interest $E_{\mathrm{c}}$.) Instead of Eq. (D6) we now have the $1 / N$ expansion

$$
\left\langle\tau_{k}\right\rangle=\left\langle\tau_{k}\right\rangle_{0}+N^{-1} \delta \tau_{k}+\mathcal{O}\left(N^{-2}\right)
$$

which contains also a term of order $N^{-1}$ because of the presence of time-reversal symmetry. The $1 / N$ expansion of $\left\langle G_{\mathrm{NS}}\right\rangle$ becomes

$$
\begin{aligned}
\frac{h}{4 e^{2}}\left\langle G_{\mathrm{NS}}\right\rangle= & \frac{N\left\langle\tau_{1}\right\rangle_{0}}{2-\left\langle\tau_{1}\right\rangle_{0}}+\frac{2 \delta \tau_{1}}{\left(2-\left\langle\tau_{1}\right\rangle_{0}\right)^{2}} \\
& +\frac{2\left\langle\tau_{1}\right\rangle_{0}^{2}-2\left\langle\tau_{1}\right\rangle_{0}^{3}-2\left\langle\tau_{2}\right\rangle_{0}+2\left\langle\tau_{1}\right\rangle_{0}\left\langle\tau_{2}\right\rangle_{0}}{\left\langle\tau_{1}\right\rangle_{0}\left(2-\left\langle\tau_{1}\right\rangle_{0}\right)^{3}} \\
& +\mathcal{O}\left(N^{-1}\right) .
\end{aligned}
$$

For the application to a disordered wire we use again Eq. (D8) for the moments $\left\langle\tau_{k}\right\rangle_{0}$, which do not depend on whether time-reversal symmetry is broken or not. We also need $\delta \tau_{1}$, which in the presence of time-reversal symmetry is given by

$$
\delta \tau_{k}=\int_{0}^{\infty} \frac{\delta \rho(x) d x}{\cosh ^{2 k} x} \Rightarrow \delta \tau_{1}=-\frac{1}{3} .
$$

Substitution into Eq. (D11) yields $\delta G_{\mathrm{NS}}=-\frac{4}{3} e^{2} / h$, see Table III.

\section{Both symmetries broken}

In the absence of both time-reversal symmetry and electron-hole degeneracy, the two matrices $u(e V)$ and $u(-e V)$ are independent, each with a uniform distribution in $\mathcal{U}(N)$. Carrying out the two averages over $\mathcal{U}(N)$ we find

$$
\frac{h}{4 e^{2}}\left\langle G_{\mathrm{NS}}\right\rangle_{u}=\frac{N \tau_{1+} \tau_{1-}}{\tau_{1+}+\tau_{1-}-\tau_{1+} \tau_{1-}} .
$$

The average over the transmission eigenvalues becomes

$$
\frac{h}{4 e^{2}}\left\langle G_{\mathrm{NS}}\right\rangle=\frac{N\left\langle\tau_{1}\right\rangle_{0}}{2-\left\langle\tau_{1}\right\rangle_{0}}+\mathcal{O}\left(N^{-1}\right),
$$

where we have used that $\delta \tau_{1}=0$ in the absence of timereversal symmetry. We conclude that $\delta G_{\mathrm{NS}}=0$ in this case, as indicated in Table III.

\section{Effect of spin-orbit scattering}

In the presence of spin-orbit scattering, the scattering matrix elements are quaternion numbers. Since a quaternion can be represented by a $2 \times 2$ matrix, we can represent the $N \times N$ matrix $V$ with quaternion elements by a $2 N \times 2 N$ matrix $v$ with complex elements. We denote this representation by $V \cong v$. In view of the definitions (271) and (272) of complex conjugation and Hermitian conjugation, one has

$$
V^{*} \cong-\mathcal{C} v^{*} \mathcal{C}, \quad V^{\prime} \cong v^{\prime},
$$

where $\mathcal{C}$ was defined in Eq. (73). In this notation, the conductance is given by

$$
\begin{aligned}
G_{\mathrm{NS}}= & 2 G_{0} \operatorname{Tr} m(e V) m^{\dagger}(e V), \quad G_{0}=e^{2} / h, \quad \text { (D16a) } \\
m(\varepsilon)= & \sqrt{\mathcal{T} \varepsilon)}\left[1-u(\varepsilon) \sqrt{\mathcal{R}(-\varepsilon)} u^{*}(-\varepsilon) \sqrt{\mathcal{R}(\varepsilon)}\right]^{-1} \\
& \times u(\varepsilon) \sqrt{\mathcal{T}(-\varepsilon),} \\
u(\varepsilon) \equiv & v^{\prime}(\varepsilon) \mathcal{C} v^{*}(-\varepsilon) .
\end{aligned}
$$

The conductance quantum $G_{0}$ is half as small as in the absence of spin-orbit scattering, while the dimensionality of the matrices $\mathcal{T}$ and $\mathcal{R}=1-\mathcal{T}$ of transmission and reflection eigenvalues has doubled. Furthermore, the term $1+u$ in Eq. (D1) is replaced by $1-u$ in Eq. (D16b), as a result of the minus sign in the definition (D15) of complex conjugation. The calculations of the previous subsections can now be repeated starting from Eq. (D16) instead of from Eq. (D1). The result is that each entry in Table III is to be multiplied by a factor of $-1 / 2$ (Slevin, Pichard, and Mello, 1996). 


\section{REFERENCES}

Abrahams, E., P. W. Anderson, D. C. Licciardello, and T. V. Ramakrishnan, 1979, Phys. Rev. Lett. 42, 673.

Abrikosov, A. A., 1981, Solid State Commun. 37, 997.

Agrait, N., J. G. Rodrigo, and S. Vieira, 1992, Phys. Rev. B 46, 5814.

Akamann, G., and J. Ambjorn, 1996, J. Phys. A 29, L555.

Aleiner, I. L., and L. I. Glazman, 1996, Phys. Rev. Lett. 77, 2057.

Alemer, I. L., and A. I. Larkin, 1997, Phys. Rev. E 55, 1243.

Alhassid, Y., and H. Attias, 1996, Phys. Rev. Lett. 76, 1711.

Alhassid, Y., and C. H. Lewenkopf, 1995, Phys. Rev. Lett. 75, 3922.

Allsopp, N. K., J. Sanchez Canizares, R. Raimondi, and C. J. Lamber1, 1996, J. Phys., Condens. Matter. 8, L377.

Altland, A., 1991, Z. Phys. B 82, 105.

Altland, A., and M. R. Zirnbauer, 1996, Phys. Rev. Lett. 76, 3420.

Altland, A., and M. R. Zirnbauer, 1997, Phys. Rev. B 55, 1142.

Altshuler, B. L., 1985, Pis'ma Zh. Eksp. Teor. Fiz. 41, 530

[JETP Lett. 41, 648].

Altshuler, B. L., and A. G. Aronov, 1985, in Electron-Electron Interactions in Disordered Systems, edited by A. L. Efros and M. Pollak (North-Holland, Amsterdam), p. 1.

Altshuler, B. L., Y. Gefen, A. Kamenev, and L. S. Levitov, 1997, Phys. Rev. Lett. 78, 2803.

Altshuler, B. L., D. E. Khmel'nitskiŭ, and B. Z. Spivak, 1983, Solid State Commun. 48, 841 .

Altshuler, B. L., P. A. Lee, and R. A. Webb, Eds., 1991, Mesoscopic Phenomena in Solids (North-Holland, Amsterdam), p. 1.

Altshuler, B. L., L. S. Levitov, and A. Yu. Yakovets, 1994, Pis'ma Zh. Eksp. Teor. Fiz. 59, 821 [JETP Lett. 59, 857].

Altshuler, B. L., and B. I. Shklovskiǐ, 1986, Zh. Eksp. Teor. Fiz. 91, 220 [Sov. Phys. JETP 64, 127].

Altshuler, B. L., and B. D. Simons, 1995, in Mesoscopic Quantum Physics, edited by E. Akkermans, G. Montambaux, J.-L. Pichard, and J. Zinn-Justin (North-Holland, Amsterdam).

Altshuler, B. L., and B. Z. Spivak, 1987, Zh. Eksp. Teor. Fiz. 92, 609 [Sov. Phys. JETP 65, 343].

Ambjørn, J., J. Jurkiewicz, and Yu. M. Makeenko, 1990, Phys. Lett. B 251, 517.

Ambjørn, J., and Yu. M. Makeenko, 1990, Mod. Phys. Lett. A 5,1753 .

Anantram, M. P., and S. Datta, 1996, Phys. Rev. B 53, 16390.

Anderson, P. W., E. Abrahams, and T. V. Ramakrishnan, 1979, Phys. Rev. Lett. 43, 718.

Anderson, P. W., D. J. Thouless, E. Abrahams, and D. S. Fisher, 1980, Phys. Rev. B 22, 3519.

Andreev, A. F., 1964, Zh. Eksp. Teor. Fiz. 46, 1823 [Sov. Phys. JETP 19, 1228].

Andreev, A. F., 1966, Zh. Eksp. Teor. Fiz. 51, 1510 [Sov. Phys. JETP 24, 1019].

Andreev, A. V., O. Agam, B. D. Simons, and B. L. Altshuler, 1996, Phys. Rev. Lett. 76, 3947.

Argaman, N., 1995, Phys. Rev. Lett. 75, 2750.

Argaman, N., 1996, Phys. Rev. B 53, 7035.

Argaman, N., 1997, Los Alamos preprint archive, cond-mat/9608084.

Argaman, N., Y. Imry, and U. Smilansky, 1993, Phys. Rev. B 47, 4440 .

Argaman, N., and A. Zee, 1996, Phys. Rev. B 54, 7406.
Artemenko, S. N., A. F. Volkov, and A. V. Zautsev, 1979, Solid State Commun. 30, 771.

Ashcroft, N. W., and N. D. Mermin, 1976, Solid State Physics (Holt, Rinehart and Winston, New York).

Averin, D. V., A. N. Korotkov, and K. K. Likharev, 1991, Phys. Rev. B 44, 6199.

Averin, D. V., and K. K. Likharev, 1991, in Mesoscopic Phenomena in Solids, edited by B. L. Altshuler, P. A. Lee, and R. A. Webb (North-Holland, Amsterdam), p. 173.

Averin, D. V., and Yu. V. Nazarov, 1990, Phys. Rev. Lett. 65, 2446.

Bakker, S. J. M., E. van der Drift, T. M. Klapwijk, H. M. Jaeger, and S. Radelaar, 1994, Phys. Rev. B 49, 13275.

Balents, L., and M. P. A. Fisher, 1996, Phys. Rev. Lett. 76, 2782.

Balents, L., M. P. A. Fisher, and M. R. Zirnbauer, 1997, Los Alamos preprint archive, cond-mat/9608049.

Balian, R., 1968, Nuovo Cimento B 57, 183.

Baranger, H. U., 1996, in Nano-Science and Technology, edited by G. Timp (American Institute of Physics, New York).

Baranger, H. U., R. A. Jalabert, and A. D. Stone, 1993a, Phys. Rev. Lett. 70, 3876.

Baranger, H. U., R. A. Jalabert, and A. D. Stone, 1993b, CHAOS 3, 665.

Baranger, H. U., and P. A. Mello, 1994, Phys. Rev. Lett. 73, 142.

Baranger, H. U., and P. A. Mello, 1995, Phys. Rev. B 51, 4703. Baranger, H. U., and P. A. Mello, 1996a, Europhys. Lett. 33, 465.

Baranger, H. U., and P. A. Mello, 1996b, Phys. Rev. B 54, 14297.

Basor, E. L., and C. A. Tracy, 1993, J. Stat. Phys. 73, 415.

Beenakker, C. W. J., 1991, Phys. Rev. B 44, 1646.

Beenakker, C. W. J., 1992a, Phys. Rev. B 46, 12841.

Beenakker, C. W. J., 1992b, in Transport Phenomena in Mesoscopic Systems, edited by H. Fukuyama and T. Ando (Springer, Berlin), p. 235.

Beenakker, C. W. J., 1993a, Phys. Rev. Lett. 70, 1155.

Beenakker, C. W. J., 1993b, Phys. Rev. Lett. 70, 4126.

Beenakker, C. W. J., 1993c, Phys. Rev. B 47, 15763.

Beenakker, C. W. J., 1994a, Nucl. Phys. B 422, 515.

Beenakker, C. W. J., 1994b, Phys. Rev. B 49, 2205.

Beenakker, C. W. J., 1995, in Mesoscopic Quantum Physics, edited by E. Akkermans, G. Montambaux, J.-L. Pichard, and J. Zinn-Justin (North-Holland, Amsterdam), p. 279.

Beenakker, C. W. J., and M. Büttiker, 1992, Phys. Rev. B 46, 1889.

Beenakker, C. W. J., and J. A. Melsen, 1994, Phys. Rev. B 50, 2450.

Beenakker, C. W. J., J. A. Melsen, and P. W. Brouwer, 1995, Phys. Rev. B 51, 13883.

Beenakker, C. W. J., J. C. J. Paasschens, and P. W. Brouwer, 1996, Phys. Rev. Lett. 76, 1368.

Beenakker, C. W. J., and B. Rejaei, 1993, Phys. Rev. Lett. 71, 3689.

Beenakker, C. W. J., and B. Rejaei, 1994a, Phys. Rev. B 49, 7499.

Beenakker, C. W. J., and B. Rejaei, 1994b, Physica A 203, 61. Beenakker, C. W. J., B. Rejaei, and J. A. Melsen, 1994, Phys. Rev. Lett. 72, 2470.

Beenakker, C. W. J., and H. van Houten, 1991, Solid State Phys. 44, 1. 
Belitz, D., and T. R. Kirkpatrick, 1994, Rev. Mod. Phys. 66, 261.

Bergmann, G., 1984, Phys. Rep. 107, 1.

Berkovits, R., and S. Feng, 1992, Phys. Rev. B 45, 97.

Berkovits, R., and S. Feng, 1994, Phys. Rep. 238, 135.

Berry, M. V., 1985, Proc. R. Soc. London 400, 229.

Birge, N. O., B. Golding, and W. H. Maemmerle, 1989, Phys. Rev. Lett. 62, 195.

Blonder, G. E., M. Tinkham, and T. M. Klapwijk, 1982, Phys. Rev. B 25, 4515.

Blümel, R., and U. Smilansky, 1988, Phys. Rev. Lett. 60, 477. Blümel, R., and U. Smilansky, 1990, Phys. Rev. Lett. 64, 241. Bohigas, O., 1990, in Chaos and Quantum Physics, edited by M.-J. Giannoni, A. Voros, and J. Zinn-Justin (North-Holland, Amsterdam), p. 87.

Bohigas, O., M.-J. Giannoni, A. M. Ozorio de Almeida, and C. Schmit, 1995, Nonlinearity 8, 203.

Bohigas, O., M.-J. Giannoni, and C. Schmit, 1984, Phys. Rev. Lett. 52, 1.

Boutet de Monvel, A., L. Pastur, and M. Shcherbina, 1995, J. Stat. Phys. 79, 585.

Brézin, E., and A. Zee, 1993, Nucl. Phys. B 402, 613.

Brézin, E., and A. Zee, 1994, Phys. Rev. E 49, 2588.

Brezini, A., and N. Zekri, 1992, Phys. Status Solidi B 169, 253.

Brody, T. A., J. Flores, J. B. French, P. A. Mello, A. Pandey, and S. S. M. Wong, 1981, Rev. Mod. Phys. 53, 385.

Brouwer, P. W., 1994, unpublished.

Brouwer, P. W., 1995, Phys. Rev. B 51, 16878.

Brouwer, P. W., 1997, Ph.D. thesis (Leiden University, unpublished).

Brouwer, P. W., and C. W. J. Beenakker, 1994, Phys. Rev. B 50, 11263.

Brouwer, P. W., and C. W. J. Beenakker, 1995a, Phys. Rev. B 51, 7739.

Brouwer, P. W., and C. W. J. Beenakker, 1995b, Phys. Rev. B 52, 3868 .

Brouwer, P. W., and C. W. J. Beenakker, 1995c, Phys. Rev. B 52, 16772.

Brouwer, P. W., and C. W. J. Beenakker, 1996a, J. Math. Phys. (N.Y.) 37, 4904.

Brouwer, P. W., and C. W. J. Beenakker, 1996b, Phys. Rev. B 54, 12705.

Brouwer, P. W., and C. W. J. Beenakker, 1997a, Phys. Rev. B 55, 4695 .

Brouwer, P. W., and C. W. J. Beenakker, 1997b, Chaos, Solitons and Fractals (in press).

Brouwer, P. W., and M. Büttiker, 1997, Europhys. Lett. 37, 441.

Brouwer, P. W., and K. M. Frahm, 1996, Phys. Rev. B 53, 1490.

Bruce, N. A., and J. T. Chalker, 1996, J. Phys. A 29, 3761.

Bruun, J., V. C. Hui, and C. J. Lambert, 1994, Phys. Rev. B 49, 4010.

Bruus, H., C. H. Lewenkopf, and E. R. Mucciolo, 1996, Phys. Rev. B 53, 9968.

Büttiker, M., 1986a, Phys. Rev. B 33, 3020.

Büttiker, M., 1986b, Phys. Rev. B 57, 1761.

Büttiker, M., 1988a, IBM J. Res. Dev. 32, 63.

Büttiker, M., IBM J. Res. Dev. 32, 317.

Büttiker, M., 1990, Phys. Rev. Lett. 65, 2901.

Büttiker, M., 1992, Phys. Rev. B 46, 12485.

Büttiker, M., 1993, J. Phys. Condens. Matter 5, 9361.
Büttiker, M., and T. Christen, 1996, in Quantum Transport in Semiconductor Submicron Structures, edited by B. Kramer, NATO ASI Series E326 (Kluwer, Dordrecht), p. 263.

Büttiker, M., A. Prêtre, and H. Thomas, 1993, Phys. Rev. Lett. 70, 4114.

Calogero, F., 1969, J. Math. Phys. (N.Y.) 10, 2191.

Caselle, M., 1995, Phys. Rev. Lett. 74, 2776.

Chalker, J. T., and M. Bernhardt, 1993, Phys. Rev. Lett. 70, 982.

Chalker, J. T., and A. Dohmen, 1995, Phys. Rev. Lett. 75, 4496. Chalker, J. T., and A. M. S. Macêdo, 1993, Phys. Rev. Lett. 71, 3693.

Chan, I. H., R. M. Clarke, C. M. Marcus, K. Campman, and A. C. Gossard, 1995, Phys. Rev. Lett. 74, 3876.

Chang, A. M., H. U. Baranger, L. N. Pfeiffer, and K. W. West, 1994, Phys. Rev. Lett. 73, 2111.

Chang, A. M., H. U. Baranger, L. N. Pfeiffer, K. W. West, and T. Y. Chang, 1996, Phys. Rev. Lett. 76, 1695.

Charlat, P., H. Courtois, Ph. Gandit, D. Mailly, A. F. Volkov, and B. Pennetier, 1996, Phys. Rev. Lett. 77, 4950.

Chen, Y., M. E. H. Ismail, and K. A. Muttalib, 1992, J. Phys., Condens. Matter. 4, L417.

Cho, S., and M. P. A. Fisher, 1997, Phys. Rev. B 55, 1637.

Claughton, N. R., R. Raimondi, and C. J. Lambert, 1996, Phys.

Rev. B 53, 9310.

Cobden, D. H., and E. Kogan, 1996, Phys. Rev. B 54, 17316.

Courtois, H., Ph. Gandit, D. Mailly, and B. Pannetier, 1996, Phys. Rev. Lett. 76, 130.

Creutz, M., 1978, J. Math. Phys. (N.Y.) 19, 2043.

Crisanti, A., G. Paladin, and A. Vulpiani, 1993, Products of Random Matrices in Statistical Physics (Springer, Berlin).

Datta, S., 1995, Electronic Transport in Mesoscopic Systems (Cambridge University, Cambridge, England).

Debray, P., J.-L. Pichard, J. Vicente, and P. N. Tung, 1989, Phys. Rev, Lett. 63, 2264.

De Gennes, P. G., 1966, Superconductivity of Metals and Alloys (Benjamin, New York).

De Jong, M. J. M., 1994, Phys. Rev. B 49, 7778.

De Jong, M. J. M., and C. W. J. Beenakker, 1992, Phys. Rev. B 46, 13400.

De Jong, M. J. M., and C. W. J. Beenakker, 1994, Phys. Rev. B 49, 16070.

De Jong, M. J. M., and C. W. J. Beenakker, 1995, Phys. Rev. B 51, 16867.

De Jong, M. J. M., and C. W. J. Beenakker, 1996, Physica A 230, 219.

De Jong, M. J. M., and C. W. J. Beenakker, 1997, in Mesoscopic Electron Transport, edited by L. P. Kouwenhoven, G. Schön, and L. L. Sohn, NATO ASI Series E (Kluwer, Dordrecht), in press.

Den Hartog, S. G., C. M. A. Kapteyn, B. J. van Wees, T. M. Klapwijk, W. van der Graaf, and G. Borghs, 1996, Phys. Rev. Lett. 76, 4592.

De Vegvar, P. G. N., T. A. Fulton, W. H. Mallison, and R. E. Miller, 1994, Phys. Rev. Lett. 73, 1416.

Dimoulas, A., J. P. Heida, B. J. van Wees, T. M. Klapwijk, W. van de Graaf, and G. Borghs, 1995, Phys. Rev. Lett. 74, 602. Dorokhov, O. N., 1982, Pis'ma Zh. Eksp. Teor. Fiz. 36, 259 [JETP Lett. 36, 318].

Dorokhov, O. N., 1983, Zh. Eksp. Teor. Fiz. 85, 1040 [Sov. Phys. JETP 58, 606].

Dorokhov, O. N., 1984, Solid State Commun. 51, 381.

Dorokhov, O. N., 1988, Phys. Rev. B 37, 10526. 
Dorokhov, O. N., 1990, Zh. Eksp. Teor. Fiz. 98, 646 [Sov. Phys. JETP 71, 360].

Doron, E., and U. Smilansky, 1992, Nucl. Phys. A 545, 455c.

Dupuis, N., and G. Montambaux, 1991, Phys. Rev. B 43, 14390.

Dyson, F. J., 1962a, J. Math. Phys. (N.Y.) 3, 140.

Dyson, F. J., 1962b, J. Math. Phys. (N.Y.) 3, 157.

Dyson, F. J., 1962c, J. Math. Phys. (N.Y.) 3, 1191.

Dyson, F. J., 1962d, J. Math. Phys. (N.Y.) 3, 1199.

Dyson, F. J., 1972, J. Math. Phys. (N.Y.) 13, 90.

Dyson, F. J., and M. L. Mehta, 1963, J. Math. Phys. (N.Y.) 4, 701.

Edwards, J. T., and D. J. Thouless, 1972, J. Phys. C 5, 807.

Efetov, K. B., 1982, Zh. Eksp. Teor. Fiz. 83, 833 [Sov. Phys. JETP 56, 467].

Efetov, K. B., 1983, Adv. Phys. 32, 53.

Efetov, K. B., 1995, Phys. Rev. Lett. 74, 2299.

Efetov, K. B., 1996, Supersymmetry in Disorder and Chaos

(Cambridge University, Cambridge, England).

Efetov, K. B., and A. I. Larkin, 1983, Zh. Eksp. Teor. Fiz. 85, 764 [Sov. Phys. JETP 58, 444].

Elattari, B., V. Kagalovsky, and H. A. Weidenmüller, 1996,

Nucl. Phys. A 606, 86.

Endesfelder, D., 1996, Phys. Rev. B 53, 16555.

Endesfelder, D., and B. Kramer, 1993, Phys. Rev. E 48, 3225.

Erdös, P., and R. C. Herndon, 1982, Adv. Phys. 31, 65.

Eynard, B., 1994, Los Alamos preprint archive, hep-th/9401165.

Fal'ko, V. I., 1995, Phys. Rev. B 51, 5227.

Fertig, H. A., and S. Das Sarma, 1989, Phys. Rev. B 40, 7410.

Fisher, D. S., and P. A. Lee, 1981, Phys. Rev. B 23, 6851.

Folk, J. A., S. R. Patel, S. F. Godijn, A. G. Huibers, S. M. Cronewett, C. M. Marcus, K. Campman, and A. C. Gossard, 1996, Phys. Rev. Lett. 76, 1699.

Forrester, P. J., 1995, Nucl. Phys. B 435, 421.

Frahm, K. M., 1995a, Phys. Rev. Lett. 74, 4706.

Frahm, K. M., 1995b, Europhys. Lett. 30, 457.

Frahm K. M., P. W. Brouwer, J. A. Melsen, and C. W. J. Beenakker, 1996, Phys. Rev. Lett. 76, 2981.

Frahm, K. M., and A. Müller-Groeling, 1996, J. Phys. A 29, 5313.

Frahm, K. M., and J.-L. Pichard, 1995a, J. Phys. (France) I 5, 847.

Frahm, K. M., and J.-L. Pichard, 1995b, J. Phys. (France) I 5, 877.

Freidrig, H., and D. Wintgen, 1989, Phys. Rep. 183, 37.

Freilikher, V., E. Kanzieper, and I. Yurkevich, 1996, Phys. Rev. E 53, 2200.

Freilikher, V., M. Pustilnik, and I. Yurkevich, 1994, Phys. Rev. Lett. 73, 810 .

Friedman, W. A., and P. A. Mello, 1985a, Ann. Phys. (N.Y.) 161, 276.

Friedman, W. A., and P. A. Mello, 1985b, J. Phys. A 18, 425.

Fyodorov, Y. V., and A. D. Mirlin, 1991, Phys. Rev. Lett. 67, 2405.

Fyodorov, Y. V., and A. D. Mirlin, 1994, Int. J. Mod. Phys. B 8, 3795.

Gazaryan, Yu. L., 1969, Zh. Eksp. Teor. Fiz. 56, 1856 [Sov. Phys. JETP 29, 996].

Gertsenshtein, M. E., and V. B. Vasil'ev, 1959, Teor. Veroyatn. Primen. 4, 424; 5, 3(E) [Theor. Probab. Appl. 4, 391; 5, 340(E)].

Ghenim, L., D. L. Sivco, A. Y. Cho, and G. Hill, 1996, Phys. Rev. B 54, 11479.
Goldberg, J., U. Smilansky, M. V. Berry, W. Schweizer, G. Wunner, and G. Zeller, 1991, Nonlinearity 4, 1.

Golubov, A. A., and M. Yu. Kupriyanov, 1995, Pis'ma Zh. Eksp. Teor. Fiz. 61, 830 [JETP Lett. 61, 851].

Golubov, A. A., F. K. Wilhelm, and A. D. Zaikin, 1997, Phys. Rev. B 55, 1123.

Gopar, V. A., M. Martinez, and P. A. Mello, 1995, Phys. Rev. B 51, 16917.

Gopar, V. A., M. Martínez, P. A. Mello, and H. U. Baranger, 1996, J. Phys. A 29, 881.

Gopar, V. A., P. A. Mello, and M. Büttiker, 1996, Phys. Rev. Lett. 77, 3005.

Gor'kov, L. P., and G. M. Eliashberg, 1965, Zh. Eksp. Teor. Fiz. 48, 1407 [Sov. Phys. JETP 21, 940].

Gor'kov, L. P., A. I. Larkin, and D. E. Khmel'nitskiǔ, 1979, Pis'ma Zh. Eksp. Teor. Fiz. 30, 248 [JETP Lett. 30, 228].

Gruzberg, I. A., N. Read, and S. Sachdev, 1997, Los Alamos preprint archive, cond-mat/9612038.

Guhr, T., 1996, Phys. Rev. Lett. 76, 2258.

Gutzwiller, M. C., 1990, Chaos in Classical and Quantum Mechanics (Springer, Berlin).

Haake, F., 1992, Quantum Signatures of Chaos (Springer, Berlin).

Hackenbroich, G., and H. A. Weidenmüller, 1995, Phys. Rev. Lett. 74, 4118.

Hamermesh, M., 1962, Group Theory and its Applications to Physical Problems (Addison-Wesley, Reading, MA).

Hastings, H. B., A. D. Stone, and H. U. Baranger, 1994, Phys. Rev. B 50, 8230.

Hekking, F. W. J., and Yu. V. Nazarov, 1993, Phys. Rev. Lett. 71, 1625.

Hekking, F. W. J., and Yu. V. Nazarov, 1994, Phys. Rev. B 49, 6847.

Holweg, P. A. M., J. A. Kokkedee, J. Caro, A. H. Verbruggen, S. Radelaar, A. G. M. Jansen, and P. Wyder, 1991, Phys. Rev. Lett. 67, 2549.

Hua, L. K., 1963, Harmonic Analysis of Functions of Several Complex Variables in the Classical Domains (American Mathematical Society, Providence).

Huckestein, B., 1995, Rev. Mod. Phys. 67, 357.

Hüffmann, A., 1990, J. Phys. A 23, 5733.

Iida, S., H. A. Weidenmüller, and J. A. Zuk, 1990a, Phys. Rev. Lett. 64, 583.

Iida, S., H. A. Weidenmüller, and J. A. Zuk, 1990b, Ann. Phys. (N.Y.) 200, 219.

Imry, Y., 1986a, Europhys. Lett. 1, 249.

Imry, Y., 1986b, in Directions in Condensed Matter Physics, edited by G. Grinstein and G. Mazenko (World Scientific, Singapore), p. 101.

Imry, Y., 1995, in Mesoscopic Quantum Physics, edited by E. Akkermans, G. Montambaux, J.-L. Pichard, and J. ZinnJustin (North-Holland, Amsterdam), p. 181.

Imry, Y., 1996, Introduction to Mesoscopic Physics (Oxford University, London).

Ishio, H., 1995, J. Phys. A 28, L469.

Itzykson, C., and J.-B. Zuber, 1980, Quantum Field Theory (McGraw-Hill, New York).

Jalabert, R. A., H. U. Baranger, and A. D. Stone, 1990, Phys. Rev. Lett. 65, 2442.

Jalabert, R. A., and J.-L. Pichard, 1995, J. Phys. I 5, 287.

Jalabert, R. A., J.-L. Pichard, and C. W. J. Beenakker, 1993, Europhys. Lett. 24, 1. 
Jalabert, R. A., J.-L. Pichard, and C. W. J. Beenakker, 1994, Europhys. Lett. 27, 255.

Jalabert, R. A., A. D. Stone, and Y. Alhassid, 1992, Phys. Rev. Lett. 68, 3468.

Jancovici, B., and P. J. Forrester, 1994, Phys. Rev. B 50, 14599. John, S., 1984, Phys. Rev. Lett. 53, 2169.

Kadigrobov, A., A. Zagoskin, R. I. Shekhter, and M. Jonson, 1995, Phys. Rev. B 52, 8662.

Kamenev, A., and Y. Gefen, 1995, Europhys. Lett. 29, 413.

Kamien, R. D., H. D. Politzer, and M. B. Wise, 1988, Phys. Rev. Lett. 60, 1995.

Kastalsky, A., A. W. Kleinsasser, L. H. Greene, R. Bhat, F. P. Milliken, and J. P. Harbison, 1991, Phys. Rev. Lett. 67, 3026. Keldysh, L. V., 1964, Zh. Eksp. Teor. Fiz. 47, 1515 [Sov. Phys. JETP 20, 1018].

Keller, M. W., O. Millo, A. Mittal, D. E. Prober, and R. N. Sacks, 1994, Surf. Sci. 305, 501.

Keller, M. W., A. Mittal, J. W. Sleight, R. G. Wheeler, D. E. Prober, R. N. Sacks, and H. Shtrikmann, 1996, Phys. Rev. B 53, 1693.

Khlus, V. A., 1987, Zh. Eksp. Teor. Fiz. 93, 2179 [Sov. Phys. JETP 66, 1243].

Khmel'nitski1̌, D. E., 1984, Physica B 126, 235.

Kirkman, P. D., and J. B. Pendry, 1984, J. Phys. C 17, 5707.

Klapwijk, T. M., 1994, Physica B 197, 481.

Kobayakawa, T. S., Y. Hatsugai, M. Kohmoto, and A. Zee, 1995, Phys. Rev. E 51, 5365.

Kohler, W., and G. C. Papanicolaou, 1976, SIAM (Soc. Ind. Appl. Math.) J. Appl. Math. 30, 263.

Kozub, V. I., J. Caro, and P. A. M. Holweg, 1996, Physica B 218, 89.

Kozub, V. I., and A. M. Rudin, 1995, Phys. Rev, B 52, 7853.

Kramer, B., and A. MacKinnon, 1993, Rep. Prog. Phys. 56, 1469.

Krieger, T. J., 1965, Ann. Phys. (N.Y.) 42, 375.

Kulik, I. O., and A. N. Omel'yanchuk, 1984, Fiz. Nizk. Temp. 10, 305 [Sov. J. Low Temp. Phys. 10, 158].

Kulik, I. O., and R. I. Shekhter, 1975, Zh. Eksp. Teor. Fiz. 68, 623 [Sov. Phys. JETP 41, 308].

Kumar, N., 1985, Phys. Rev. B 31, 5513.

Lagendijk, A., and B. A. van Tiggelen, 1996, Phys. Rep. 270, 143.

Lambert, C. J., 1991, J. Phys., Condens. Matter. 3, 6579.

Lambert, C. J., 1993, J. Phys., Condens. Matter. 5, 707.

Lambert, C. J., V. C. Hui, and S. J. Robinson, 1993, J. Phys., Condens. Matter. 5, 4187.

Landauer, R., 1957, IBM J. Res. Dev. 1, 223.

Landauer, R., 1970, Philos. Mag. 21, 863.

Landauer, R., 1987, Z. Phys. B 68, 217.

Larkin, A. I., and Yu. V. Ovchinnikov, 1975, Zh. Eksp. Teor. Fiz. 68, 1915 [Sov. Phys. JETP 41, 960].

Larkin, A. I., and Yu. V. Ovchinnikov, 1977, Zh. Eksp. Teor. Fiz. 73, 299 [Sov. Phys. JETP 46, 155].

Lee, P. A., and T. V. Ramakrishnan, 1985, Rev. Mod. Phys. 57, 287.

Lee, P. A., and A. D. Stone, 1985, Phys. Rev. Lett. 55, 1622. Lenssen, K.-M. H., P. C. A. Jeekel, C. J. P. M. Harmans, J. E. Mooij, M. R. Leys, J. H. Wolter, and M. C. Holland, 1994, in Coulomb and Interference Effects in Small Electronic Structures, edited by D. C. Glattli, M. Sanquer, and J. Trân Thanh Vân (Editions Frontières, Gif-sur-Yvette, France), p. 63. Lenz, G., and F. Haake, 1990, Phys. Rev. Lett. 65, 2325.

Leo, J., and A. H. MacDonald, 1990, Phys. Rev. Lett. 64, 817.
Lerner, I. V., and Y. Imry, 1995, Europhys. Lett. 29, 49.

Lerner, I. V., and M. E. Raikh, 1992, Phys. Rev. B 45, 14036.

Lesovik, G. B., 1989, Pis'ma Zh. Eksp. Teor. Fiz. 49, 513 [JETP Lett. 49, 592].

Lesovik, G. B., A. L. Fauchère, and G. Blatter, 1997, Phys. Rev. B 55, 3146.

Lewenkopf, C. H., and H. A. Weidenmüller, 1991, Ann. Phys. (N.Y.) 212, 53.

Liefrink, F., J. I. Dijkhuis, M. J. M. de Jong, L. W. Molenkamp, and H. van Houten, 1994, Phys. Rev. B 49, 14066.

Likharev, K. K., 1979, Rev. Mod. Phys. 51, 101.

Macêdo, A. M. S., 1994a, Phys. Rev. B 49, 1858.

Macédo, A. M. S., 1994b, Phys. Rev. B 49, 16841.

Macêdo, A. M. S., 1996, Phys. Rev. B 53, 8411.

Macêdo, A. M. S., and J. T. Chalker, 1992, Phys. Rev. B 46, 14985.

Macêdo, A. M. S., and J. T. Chalker, 1994, Phys. Rev. B 49, 4695.

Magnée, P. H. C., N. van der Post, P. H. M. Kooistra, B. J. van Wees, and T. M. Klapwijk, 1994, Phys. Rev. B 50, 4594.

Mahaux, C., and H. A. Weidenmüller, 1969, Shell-Model Approach to Nuclear Reactions (North-Holland, Amsterdam).

Mailly, D., and M. Sanquer, 1992, J. Phys. (France) I 2, 357.

Makeenko, Yu., 1991, Mod. Phys. Lett. A 6, 1901.

Mani, R. G., L. Ghenim, and T. N. Theis, 1992, Phys. Rev. B 45, 12098.

Marcus, C. M., 1996, unpublished.

Marcus, C. M., A. J. Rimberg, R. M. Westervelt, P. F. Hopkins, and A. C. Gossard, 1992, Phys. Rev. Lett. 69, 506.

Marcus, C. M., R. M. Westervelt, P. F. Hopkins, and A. C. Gossard, 1993, Phys. Rev. B 48, 2460.

Marmorkos, I. K., C. W. J. Beenakker, and R. A. Jalabert, 1993, Phys. Rev. B 48, 2811.

Martin, Th., 1994, in Coulomb and Interference Effects in Small Electronic Structures, edited by D. C. Glattli, M. Sanquer, and J. Trân Thanh Vân (Editions Frontières, Gif-sur-Yvette, France), p. 405.

Martin, Th., 1996, Phys. Lett. A 220, 137.

Martin, Th., and R. Landauer, 1992, Phys. Rev. B 45, 1742.

Martin, Th., G. Montambaux, and J. Trân Thanh Vân, Eds. 1996, Correlated Fermions and Transport in Mesoscopic Systems (Editions Frontières, Gif-sur-Yvette, France).

Maslov, D. L., C. Barnes, and G. Kirczenow, 1993a, Phys. Rev. Lett. 70, 1984.

Maslov, D. L., C. Barnes, and G. Kirczenow, 1993b, Phys. Rev. B 48, 2543.

Maxwell, J. C., 1891, A Treatise on Electricity and Magnetism (Dover, New York).

McCann, E., and I. V. Lerner, 1996, J. Phys., Condens. Matter. 8, 6719.

Mehta, M. L., 1991, Random Matrices (Academic, New York). Mehta, M. L., and A. Pandey, 1983, J. Phys. A 16, L601.

Meir, Y., and O. Entin-Wohlman, 1993, Phys. Rev. Lett. 70, 1988.

Meir, Y., N. S. Wingreen, O. Entin-Wohlman, and B. L. Altshuler 1991, Phys. Rev. Lett. 66, 1517.

Meir, Y., N. S. Wingreen, and P. A. Lee, 1991, Phys. Rev. Lett. 66, 3048.

Meirav, U., and E. B. Foxman, 1995, Semicond. Sci. Technol. 10, 255.

Mello, P. A., 1986, J. Math. Phys. (N.Y.) 27, 2876.

Mello, P. A., 1988, Phys. Rev. Lett. 60, 1089.

Mello, P. A., 1990, J. Phys. A 23, 4061. 
Mello, P A , 1995, in Mesoscopıc Quantum Physics, edited by E Akkermans, G Montambaux, J-L Pichard, and J ZinnJustın (North-Holland, Amsterdam), p 435

Mello, P A, E Akkermans, and B Shapuro, 1988, Phys Rev Lett 61, 459

Mello, P A, P Pereyra, and N Kumar, 1988, Ann Phys (N Y ) 181, 290

Mello, P A, P Pereyra, and T H Seligman, 1985, Ann Phys (N Y) 161, 254

Mello, P A, and J -L Pichard, 1989, Phys Rev B 40, 5276

Mello, P A, and J L Pichard, 1991, J Phys (France) I 1, 493

Mello, P A, and B Shapiro, 1988, Phys Rev B 37, 5860

Mello, P A, and A D Stone, 1991, Phys Rev B 44, 3559

Mello, P A, and S Tomsovic, 1991, Phys Rev Lett 67, 342

Mello, P A, and S Tomsovic, 1992, Phys Rev B 46, 15963

Mel'nukov, V I, 1981, F1z Tverd Tela (Lenıngrad) 23, 782

[Sov Phys Solid State 23, 444]

Melsen, J A, and C W J Beenakker, 1994, Physica B 203, 219

Melsen, J A, and C W J Beenakker, 1995, Phys Rev B 51, 14483

Melsen, J A, P W Brouwer, K M Frahm, and C W J Beenakker, 1996, Europhys Lett 35, 7

Mikhlin, S G, 1964, Integral Equations (Pergamon, New York)

Millo, O, S J Klepper, M W Keller, D E Prober, S Xiong, A D Stone, and R N Sacks, 1990, Phys Rev Lett 65, 1494 Mirlın, A D, 1994, Phys Rev Lett 72, 3437

Mirlın, A D , A Muller-Groeling, and M R Zirnbauer, 1994, Ann Phys (N Y) 236, 325

Morita, Y, Y Hatsugai, and M Kohmoto, 1995, Phys Rev B 52, 4716

Mucciolo, E R, V N Prigodin, and B L Altshuler, 1995, Phys Rev B 51, 1714

Muttalib, K A, 1990, Phys Rev Lett 65, 745

Muttalıb, K A, 1995, J Phys A 28, L159

Muttalıb, K A, Y Chen, M E H Ismail, and V N Nicopoulos, 1993, Phys Rev Lett 71, 471

Muttalib, K A, J -L Pichard, and A D Stone, 1987, Phys Rev Lett 59, 2475

Muzykantskǐ, B A, and D E Khmel'nitskı̌̆, 1994, Physıca B 203, 233

Muzykantskı̌̆, B A, and D E Khmel'nıtskır, 1995, Pıs'ma Zh

Eksp Teor F1z 62, 68 [JETP Lett 62, 76]

Nagaev, K E, 1992, Phys Lett A 169, 103

Nagaev, K E, 1995, Phys Rev B 52, 4740

Nazarov, Yu V, 1994a, Phys Rev Lett 73, 134

Nazarov, Yu V, 1994b, Phys Rev Lett 73, 1420

Nazarov, Yu V, 1995a, in Quantum Dynamics of Submicron Structures, edited by H A Cerdeira, B Kramer, and G Schon, NATO ASI Series E291 (Kluwer, Dordrecht), p 687

Nazarov, Yu V, 1995b, Phys Rev B 52, 4720

Nazarov, Yu V, 1996, Phys Rev Lett 76, 2129

Nazarov, Yu V, and T H Stoof, 1996, Phys Rev Lett 76, 823

Ng, T K, and P A Lee, 1988, Phys Rev Lett 61, 1768

Nguyen, C, H Kroemer, and E L Hu, 1992, Phys Rev Lett 69, 2847

Nguyen, V I, B Z Spıvak, and B I Shklovskir, 1985, Pıs'ma

Zh Eksp Teor Fiz 41, 35 [JETP Lett 41, 42]

Nieuwenhuizen, Th M, and J M Luck, 1993, Phys Rev E 48, 569
Nishıoka, H, and H A Werdenmuller, 1985, Phys Lett B 157, 101

Oseledec, V I, 1968, Trans Moscow Math Soc 19, 197

Paasschens, J C J, T Sh Misirpashaev, and C W J Beenakker, 1996, Phys Rev B 54, 11887

Pandey, A, and M L Mehta, 1983, Commun Math Phys 87, 449

Pandey, A, and P Shukla, 1991, J Phys A 24, 3907

Papanicolaou, G C, 1971, SIAM (Soc Ind Appl Math) J Appl Math 21, 13

Pastur, L A, 1992, Lett Math Phys 25, 259

Pendry, J B, 1994, Adv Phys 43, 461

Pendry, J B , A MacKınnon, and P J Roberts, 1992, Proc R Soc London 437, 67

Pereyra, P, and P A Mello, 1983, J Phys A 16, 237

Petrashov, V T, V N Antonov, P Delsing, and T Claeson, 1995, Phys Rev Lett 74, 5268

Pichard, J -L, 1984, Ph D Thesis (University of Paris at Orsay)

Pichard, J -L , 1991, in Quantum Coherence in Mesoscopıc Systems, edited by B Kramer, NATO ASI Series B254 (Plenum, New York), p 369

Pichard, J -L, and G Andre, 1986, Europhys Lett 2, 477

Pichard, J -L, M Sanquer, K Slevin, and Ph Debray, 1990, Phys Rev Lett 65, 1812

Pichard, J -L, and G Sarma, 1981, J Phys C 14, L127

Pichard, J -L, N Zanon, Y Imry, and A D Stone, 1990, J Phys (France) 51, 587

Pluhař, Z, H A Weidenmuller, J A Zuk, and C H Lewen kopf, 1994, Phys Rev Lett 73, 2115

Pluhar, Z, H A Weidenmuller, J A Zuk, C H Lewenkopf, and F J Wegner, 1995, Ann Phys (N Y) 243, 1

Poilblanc, D, T Ziman, J Bellissard, F Mila, and G Montambaux, 1993, Europhys Lett 22, 537

Poirier, W, D Marlly, and M Sanquer, 1996, in Correlated Fermions and Transport in Mesoscoplc Systems, edited by Th Martın, G Montambaux, and J Trân Thanh Vân (Editıons Frontieres, Gif sur-Yvette, France)

Politzer, H D, 1989, Phys Rev B 40, 11917

Porter, C E, 1965, Statistical Theories of Spectra Fluctuations (Academıc, New York)

Pothier, H, S Guéron, D Esteve, and M H Devoret, 1994, Phys Rev Lett 73, 2488

Pradhan, P, and N Kumar, 1994, Phys Rev B 50, 9644

Prange, R E, and S M Girvin, Eds, 1990, The Quantum Hall Effect (Springer, New York)

Prigodin, V N, B L Altshuler, K B Efetov, and S Iıda, 1994, Phys Rev Lett 72, 546

Prigodin, V N, K B Efetov, and S Iıda, 1993, Phys Rev Lett 71, 1230

Prigodin, V N, K B Efetov, and S I1da, 1995, Phys Rev B 51, 17223

Raıkh, M E, and I M Ruzin, 1991, in Mesoscopic Phenomena in Sollds, edited by B L Altshuler, P A Lee, and R A Webb (North-Holland, Amsterdam), p 315

Rammal, R, and B Doucot, 1987, J Phys (France) 48, 509

Rau, J , 1995, Phys Rev B 51, 7734

Rejael, B , 1996, Phys Rev B 53, 13235

Samuel, S, 1980, J Math Phys (N Y ) 21, 2695

Sharvin, Yu V, 1965, Zh Eksp Teor F1z 48, 984 [Sov Phys JETP 21, 655]

Shekhter, R I, 1972, Zh Eksp Teor F1z 63, 1410 [Sov Phys JETP 36, 747 (1973)] 
Shelankov, A. L., 1980, Pis'ma Zh. Eksp. Teor. Fiz. 32, 122 [JETP Lett. 32, 111].

Shelankov, A. L., 1984, Fiz. Tverd. Tela (Leningrad) 26, 1615

[Sov. Phys. Solid State 26, 981].

Shepelyansky, D. L., 1994, Phys. Rev. Lett. 73, 2607.

Shklovskir., B. I., and B. Z. Spivak, 1990, in Hopping Conduction in Semiconductors, edited by M. Pollak and B. I. Shklovskir (North-Holland, Amsterdam).

Simons, B. D., and B. L. Altshuler, 1993, Phys. Rev. Lett. 70, 4063.

Sivan, U., Y. Imry, and A. G. Aronov, 1994, Europhys. Lett. 28, 115.

Sivan, U., F. P. Milliken, K. Milkove, S. Rishton, Y. Lee, J. M. Hong, V. Boegli, D. Kern, and M. DeFranza, 1994, Europhys. Lett. 25, 605.

Slevin, K., and T. Nagao, 1993, Phys. Rev. Lett. 70, 635.

Slevin, K., and T. Nagao, 1994, Phys. Rev. B 50, 2380.

Slevin, K., J.-L. Pichard, and P. A. Mello, 1991, Europhys. Lett. 16, 649.

Slevin, K., J.-L. Pichard, and P. A. Mello, 1996, J. Phys. (France) I 6, 529.

Slevin, K., J.-L. Pichard, and K. A. Kuttalib, 1993, J. Phys. (France) I 3, 1387.

Smilansky, U., 1990, in Chaos and Quantum Physics, edited by M.-J. Giannoni, A. Voros, and J. Zinn-Justin (North-Holland, Amsterdam), p. 371.

Spivak, B. Z., and D. E. Khmel'nitskiǔ, 1982, Pis'ma Zh. Eksp. Teor. Fiz. 35, 334 [JETP Lett. 35, 412].

Steinbach, A. H., J. M. Martinis, and M. H. Devoret, 1995, Bull. Am. Math. Soc. 40, 400.

Steinbach, A. H., J. M. Martinis, and M. H. Devoret, 1996, Phys. Rev. Lett. 76, 3806.

Stone, A. D., P. A. Mello, K. A. Muttalib, and J.-L. Pichard, 1991, in Mesoscopic Phenomena in Solids, edited by B. L. Altshuler, P. A. Lee, and R. A. Webb (North-Holland, Amsterdam), p. 369.

Stone, A. D., and A. Szafer, 1988, IBM J. Res. Dev. 32, 384.

Stoof, T.H., and Yu. V. Nazarov, 1996a, Phys. Rev. B 53, 1050. Stoof, T.H., and Yu. V. Nazarov, 1996b, Phys. Rev. B 54, 772. Sutherland, B., 1971, J. Math. Phys. (N.Y.) 12, 246.

Sutherland, B., 1972, Phys. Rev. A 5, 1372.

Szafer, A., and B. L. Altshuler, 1993, Phys. Rev. Lett. 70, 587. Szafer, A., and A. D. Stone, 1989, Phys. Rev. Lett. 62, 300.

Takane, Y., and H. Ebisawa, 1991, J. Phys. Soc. Jpn. 60, 3130. Takane, Y., and H. Ebisawa, 1992a, J. Phys. Soc. Jpn. 61, 1685. Takane, Y., and H. Ebisawa, 1992b, J. Phys. Soc. Jpn. 61, 2858. Takane, Y., and H. Ebisawa, 1993, J. Phys. Soc. Jpn. 62, 1844. Takane, Y., and H. Otani, 1994, J. Phys. Soc. Jpn. 63, 3361.

Takayanagi, H., E. Toyoda, and T. Akazaki, 1996, unpublished.

Tartakovski, A. V., 1995, Phys. Rev. B 52, 2704.

Thouless, D. J., 1977, Phys. Rev. Lett. 39, 1167.

Van Albada, M. P., B. A. van Tiggelen, A. Lagendijk, and A. Tip, 1991, Phys. Rev. Lett. 66, 3132.

Van Houten, H., C. W. J. Beenakker, and A. A. M. Staring, 1992, in Single Charge Tunneling, edited by H. Grabert and M. H. Devoret (Plenum, New York), p. 167.
Van Kampen, N. G., 1981, Stochastic Processes in Physics and Chemistry (North-Holland, Amsterdam).

Van Langen, S. A., P. W. Brouwer, and C. W. J. Beenakker, 1996, Phys. Rev. E 53, 1344.

Van Son, P. C., H. van Kempen, and P. Wyder, 1987, Phys. Rev. Lett. 59, 2226.

Van Son, P. C., H. van Kempen, and P. Wyder, 1988, J. Phys. F 18, 2211.

Van Wees, B. J., P. de Vries, P. Magnée, and T. M. Klapwijk, 1992, Phys. Rev. Lett. 69, 510.

Van Wees, B. J., and H. Takayanagi, 1997, in Mesoscopic Electron Transport, edited by L. P. Konwenhoven, G. Schön, and L. L. Sohn, NATO ASI Series E (Kluwer, Dordrecht), in press.

Verbaarschot, J. J. M., H. A. Weidenmüller, and M. R. Zirnbauer, 1985, Phys. Rep. 129, 367.

Volkov, A. F., 1994, Phys. Lett. A 187, 404.

Volkov, A. F., N. Allsopp, and C. J. Lambert, 1996, J. Phys., Condens. Matter. 8, L45.

Volkov, A. F., and A. V. Zaitsev, 1996, Phys. Rev. B 53, 9267.

Volkov, A. F., A. V. Zătsev, and T. M. Klapwijk, 1993, Physica C 210, 21.

Vollhardt, D., and P. Wölfle, 1992, in Electronic Phase Transitions, edited by W. Hanke and Yu. V. Kopaev (NorthHolland, Amsterdam), p. 1.

Wang, Z., B. Jovanović, and D.-H. Lee, 1996, Phys. Rev. Lett. $77,4426$.

Washburn, S., and R. A. Webb, 1986, Adv. Phys. 35, 375.

Wegner, F., 1979, Z. Phys. B 35, 207.

Weidenmüller, H. A., 1990, Physica A 167, 28.

Westervelt, R. M., 1996, in Nano-Science and Technology, edited by G. Timp (American Institute of Physics, New York), in press.

Wigner, E. P., 1957, in Proceedings of the Canadian Mathematical Congress (University of Toronto, Toronto), p. 174; reprinted in Porter (1965), p. 188.

Wigner, E. P., 1967, SIAM (Soc. Ind. Appl. Math.) Rev. 9, 1. Xiong, P., G. Xiao, and R. B. Laibowitz, 1993, Phys. Rev. Lett. 71, 1907.

Yang, X., H. Ishio, and J. Burgdörfer, 1995, Phys. Rev. B 52, 8219 .

Yip, S., 1995, Phys. Rev. B 52, 15504.

Yurke, B., and G. P. Kochanski, 1990, Phys. Rev. B 41, 8184.

Zaĭtsev, A. V., 1980, Zh. Eksp. Teor. Fiz. 78, 221; 79, 2016(E)

[Sov. Phys. JETP 51, 111; 52, 1018(E)].

Zaĭtsev, A. V., 1984, Zh. Eksp. Teor. Fiz. 86, 1742 [Sov. Phys. JETP 59, 1015].

Zaĭtsev, A. V., 1994, Phys. Lett. A 194, 315.

Zanon, N., and J.-L. Pichard, 1988, J. Phys. (France) 49, 907.

Zhang, Z. Q., 1995, Phys. Rev. B 52, 7960.

Zhou, F., and B. Spivak, 1996, Los Alamos preprint archive, cond-mat/9604185.

Zhou, F., B. Spivak, and A. Zyuzin, 1995, Phys. Rev. B 52, 4467.

Zirnbauer, M. R., 1992, Phys. Rev. Lett. 69, 1584.

Zirnbauer, M. R., 1993, Nucl. Phys. A 560, 95. 


\section{Information for Contributors}

Reviews of Modern Physics seeks comprehensive reviews as well as shorter colloquia in all areas of physics. For details, see "What our editors are looking for" in the January issue. We encourage prospective authors to correspond with the editors before submitting an article. Proposals should include an outline with the key citations. For regular reviews, write to George Bertsch, Editor. Reviews of Modern Physics, University of Washington, Department of Physics, Box 351560, Seattle, WA 98195-1560. E-Mail: bertsch@phys.washington.edu, and fax: (206) 543-6782. Proposals for colloquia should be directed to Professor Anthony F. Starace, Department of Physics and Astronomy, 116 Brace Laboratory, University of Nebraska, Lincoln. NE 68588-0111; E-mail: astarace@ unlinfo.unl.edu, telephone: (402) 472-2795, and fax: (402) 472-2879.

Refereeing. Submitted articles will be referred to one or more associate editors in appropriate fields. An initial decision will be made on the timeliness of the topic and breadth of appeal to our readership. Articles meeting these criteria are reviewed by outside referees, who evaluate the scientific accuracy, balance, and clarity of the manuscript. Authors can expect some additional editing in the light of these referee reports before the final manuscript is accepted.

Style and notation of submitted manuscripts should conform to the journal's standards. Authors may obtain a copy of the Reviews of Modern Physics Style Guide from the editorial office or from the RMP journal website (http://www.phys.washington.edu/ rmp/). We encourage authors to submit their manuscripts as RevTeX compuscripts. They can then be sent electronically to rmptex@aps.org. RevTeX compuscripts that meet our criteria can be typeset directly from the author's file. The file is converted to SGML format and coding, from which base the journal pages are composed.

Copyright. Submission is a representation that the article has not been published previously and is not currently submitted for publication eisewhere. A statement transferring copyright to The American Physical Society will be sent to authors by the editorial office upon acceptance of an article. The statement must be signed and returned before the article can be put into production. Requests for further copyright information should be addressed to the APS Administrative Editor, 1 Research Road, Box 9000, Ridge, NY 11961.

\section{Information for Readers}

Subscriptions for members of the American Physical Society are available at reduced rates. See the information in the inside front cover. Changes of address of APS members should be addressed to APS Membership Department, The American Physical Society, One Physics Ellipse, College Park, MD 20740-3844. Libraries and other individuals should contact Circulation and Fulfillment Division, American Institute of Physics, 500 Sunnyside Blvd., Woodbury, NY 11797. For change of address, allow at least six weeks advance notice; send both old and new addresses and, if possible, a mailing label from a recent issue. For your convenience a change of address form is included in every issue of Physics Today. Requests from subscribers for missing journal issues will be honored without charge only if received within six months of the issue's actual date of publication; otherwise, the issue may be purchased at the single-copy price from the Circulation and Fulfillment Division.

Reprints of individual articles and of supplements, bound in covers similar to that of the journal, are sold by the American Institute of Physics as a service to our readers. A searchable Reprint Archive going back to 1974 , with prices and procedures for ordering, is posted on the RMP website (http:// www.phys.washington.edu/ rmp/). A print version appears in the October issue of the journal. Our contributors receive a discount on prepublication orders for reprints of their own articles; often free reprints are available directly from them.

Back numbers are available from 1954 (Vol. 26) at a rate of $\$ 85$ per copy. Cumulative Index. For an on-line searchable index, see our website (http://www.phys.washington.edu/ $\sim \mathrm{rmp} /$ ) under Reprint Archives. Softbound printed indices are available as follows: Vols. 1-27 (1929-1955), \$20; Vols. 28-45 (1956-1973), \$20; Vols. 46-53 (1974-1981), \$25; Vols. 54-61 (1982-1989), \$25. Contact the Circulation and Fulfillment Division. Starting with 1993 the journal began including a five-year cumulative index in each October issue.

Microfilm editions of complete volumes of Reviews of Modern Physics from Vol. 1 through the present are available on 16 and $35 \mathrm{~mm}$. Reviews of Modern Physics, along with other current publications by the American Institute of Physics and its member societies, also appears in Current Physics Microform (CPM) Section I, which is published on a monthly basis. For information concerning prices and orders for microfilm and CPM, contact the Circulation and Fulfillment Division, American Institute of Physics, 500 Sunnyside Blvd., Woodbury, NY 11797. 